\title{
ULIB
}

\section{Systematic Analyses of structure/function variability of viral K+ channels for the development of synthetic channels}

\author{
Kukovetz, Kerri
}

(2020)

DOI (TUprints):

Lizenz:

https://doi.org/10.25534/tuprints-00011813

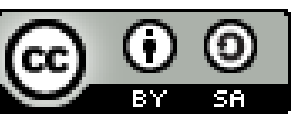

CC-BY-SA 4.0 International - Creative Commons, Attribution Share-alike

Publikationstyp:

Ph.D. Thesis

Fachbereich:

10 Department of Biology

Quelle des Originals:

https://tuprints.ulb.tu-darmstadt.de/11813 


\section{Systematic analyses of structure/function variability of viral $\mathrm{K}^{+}$channels for the development of synthetic channels}

Vom Fachbereich Biologie der Technischen Universität Darmstadt

zur Erlangung des akademischen Grades

eines Doctor rerum naturalium

Dissertation von

Kerri Kukovetz

1. Referent: Prof. Dr. Gerhard Thiel

2. Referentin: Dr. Indra Schröder

Eingereicht am: 10.06.2020

Mündliche Prüfung am: 29.07.2020

Darmstadt 2020 
Kukovetz, Kerri: Systematic analyses of structure/function variability of viral $\mathrm{K}^{+}$channels for the development of synthetic channels

Darmstadt, Technische Universität Darmstadt

Jahr der Veröffentlichung der Dissertation auf TUPrints: 2020

URN: urn:nbn:de:tuda-tuprints-118130

Tag der mündlichen Prüfung: 29.07.2020

Veröffentlicht unter CC-BY-SA 4.0 International

https://creativecommons.org/licenses/ 
Nicht Kunst und Wissenschaft allein, Geduld will bei dem Werke sein -Johann Wolfgang von Goethe 


\section{Table of contents}

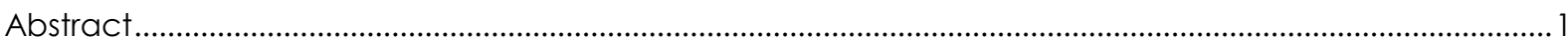

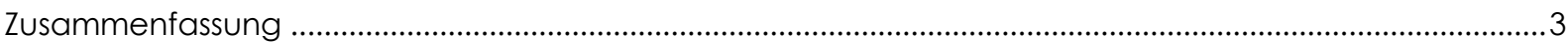

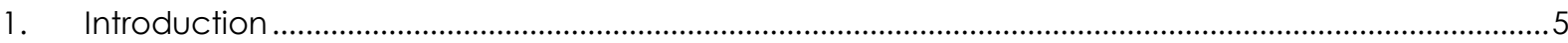

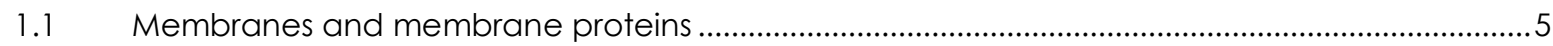

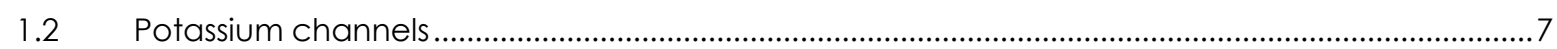

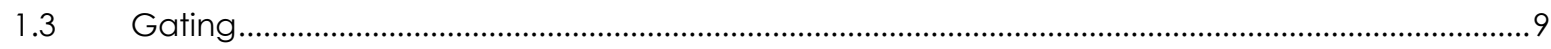

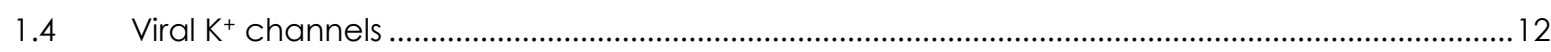

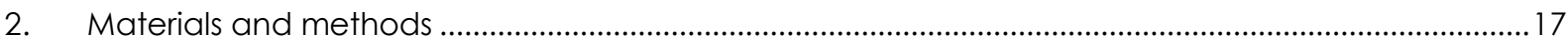

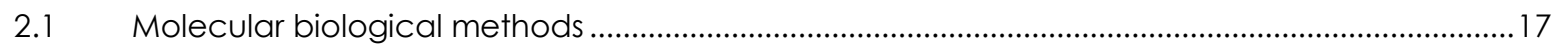

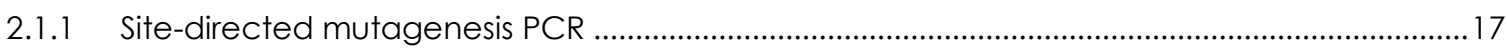

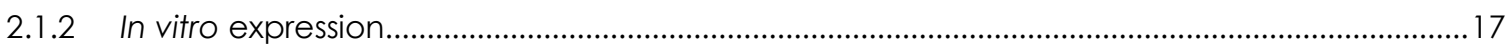

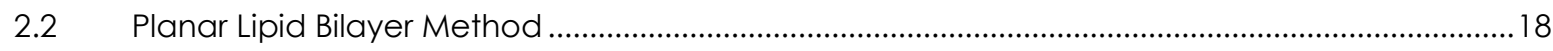

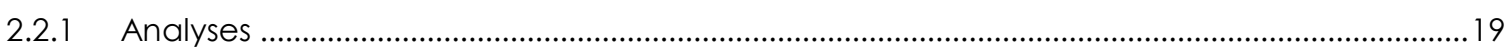

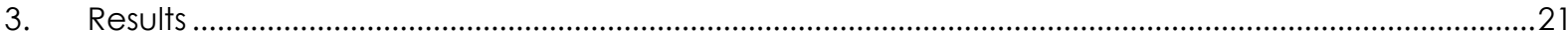

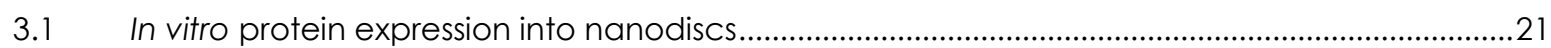

3.1.1 Target membrane properties determine channel function .........................................................22

3.1.2 Expression and successful reconstitution of a blue-light-induced $\mathrm{K}^{+}$channel (BLINK1) in PLB 27

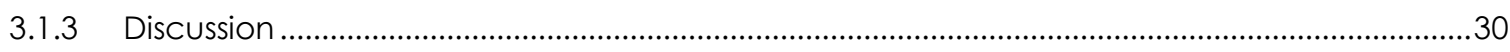

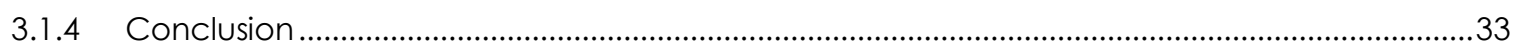

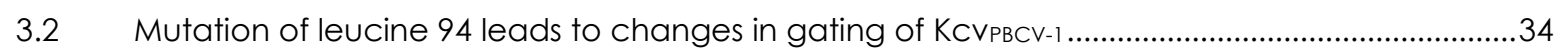

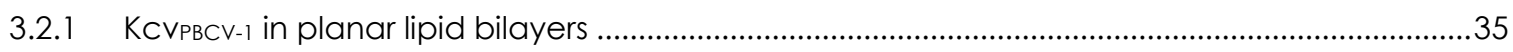

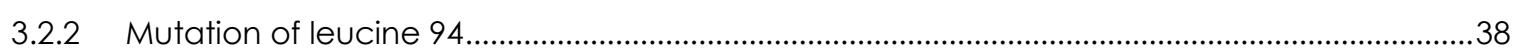

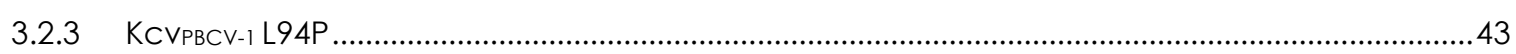

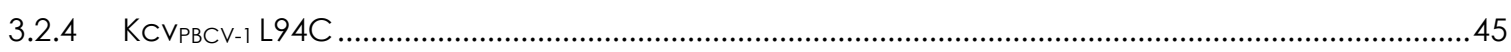

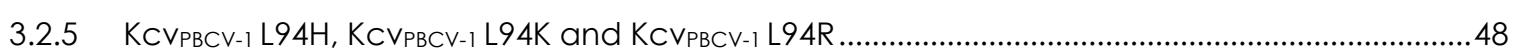

3.2.6 The effect of varying proton concentration on $\mathrm{KCVPBCV}_{-1}$ and $\mathrm{KCVPBCV}_{-1} \mathrm{L94H}$.........................50

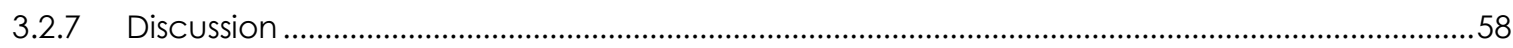

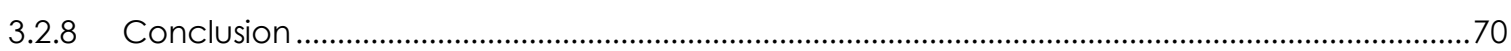

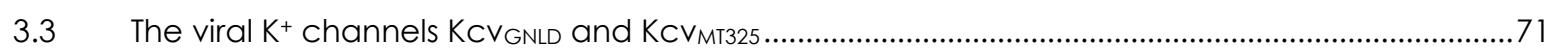

3.3.1 Mutation of threonine to valine right before the GFG motive eliminates voltage dependency

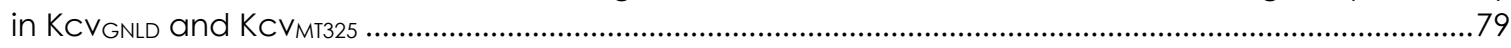

3.3.2 Effect of mutation of valine to threonine right before the GFG motive in KCVPBCV-1 ...............84

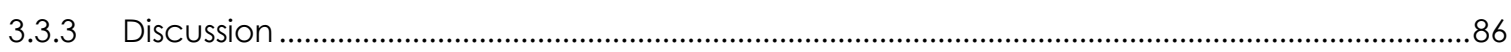

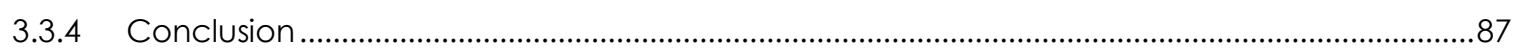

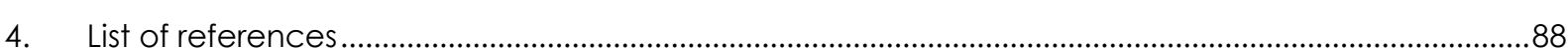

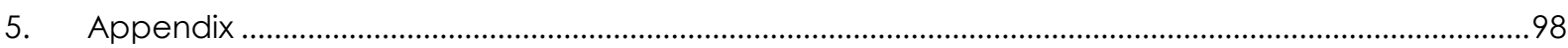

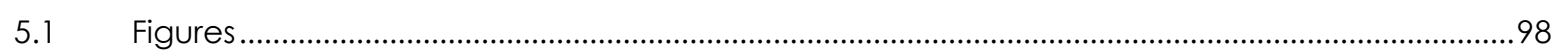

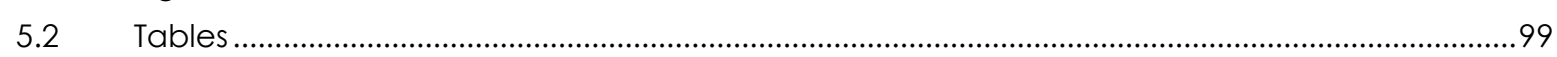

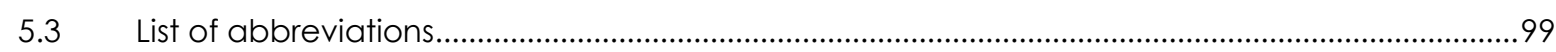

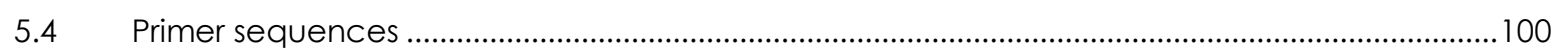

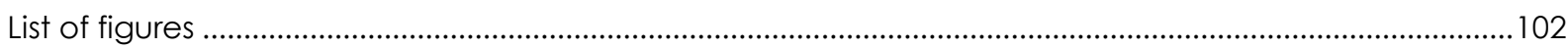


Own work. 104

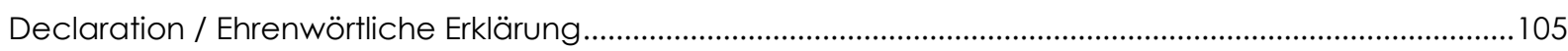

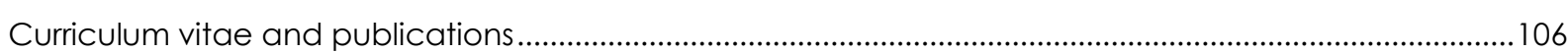

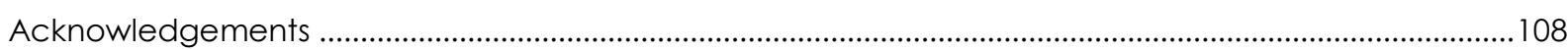




\section{Abstract}

Potassium $\left(\mathrm{K}^{+}\right)$channels are an important class of ion channels, which serve crucial physiological functions. All known $\mathrm{K}^{+}$channels have a similar architecture: a central ion-conducting pore, with a high similarity throughout all different forms of life, eukaryotes, archaea, bacteria and even in viruses. The latter turns out to be a very interesting group: $\mathrm{K}^{+}$channels isolated from viruses are reduced to an absolute minimum, basically representing the pore module of every $\mathrm{K}^{+}$channel. Despite their minimall size, they still possess many of the essential and characteristic properties of bigger and more complex channels. This makes them an ideal model system to investigate structure/function correlations that determine ion permeation and gating in $\mathrm{K}^{+}$channels.

In this study, the planar lipid bilayer (PLB) method was used to analyze small viral $\mathrm{K}^{+}$channels on the single channel level. This reduced electrophysiological measuring system allows a quick and easy modification of the experimental conditions such as $\mathrm{pH}$, ion concentration or lipid environment, as well as a straight forward addition of blockers. In the first part, a novel technique for a fast and artifact-free functional analysis is introduced. We show that adding nanodiscs to an in vitro expression system results in a timesaving and contamination-free method for expression and purification of membrane proteins, which can subsequently be used for single channel analyses with different methods. The viral potassium channel KCVNTS, as well as the model bacterial channel KcsA could successfully be reconstituted into PLBs after expression in the presence of nanodiscs. The experiments also show that not the lipid from nanodisc determines channel function, but the lipid composition of the target membrane in which the channel of interest later incorporates into. The data also show that this technique could be used for the functional reconstitution of the synthetic blue light sensitive channel BLINK1. Unlike expression in cells, in PLBs this channel loses its light sensitivity, which demonstrates a shortcoming of the method regarding posttranslational modifications.

The second part of the study is dedicated to the systematic analyses of the small viral $\mathrm{K}^{+}$channel KCVPBCV1. A subunit consists of 94 amino acids, which contains two transmembrane (TM) helices, a pore loop including the selectivity filter and a short $\mathrm{N}$-terminal helix. Previous studies with yeast complementation assays have shown that leucine at position 94 plays a crucial role in channel gating. In this preceding study, leucine was exchanged to all other proteinogenic amino acids and the degree of yeast complementation was monitored as an indirect parameter for channel activity. Here, KCVPBCV-1 and the 19 mutants KCVPBCV-1 L94X were analyzed on the single channel level, to determine the effect of each mutation on the key functional parameters of channel function: unitary single channel conductance and open probability. The single channel analyses are not compatible with the yeast complementation assays. This means that the latter method is suitable for screening of basal channel function but provides no information on detailed functional features of a channel. The results of the yeast complementation assays are presumably also influenced by secondary factors such as sorting and translation efficiency of the channel protein.

Nonetheless, the single channel data demonstrate that the last amino acid of KCV PBCV-1 $_{\text {l }}$ has a complex impact on channel function and can modulate the open probability as well as the unitary single channel conductance. The main observations are that KCVPBCV-1 L94P and KCVPBCV-1 L94C have both, an increasing 
impact on the channels open probability and voltage dependency. Another discovery was that introduction of an amino acid with a basic side chain inverts the voltage dependency of the open probability and reduces the unitary single channel conductance of KCVPBCV-1. Since the amino acid histidine can be titrated in the physiological pH range, KCVPBCV-1 and KCVPBCV-1 L94H were examined within a $\mathrm{pH}$ window from 4 to 9. It turns out that the wild type channel already exhibits a mild sensitivity toward $\mathrm{H}^{+}$, which is strongly increased by mutation of $\mathrm{L} 94 \mathrm{H}$. This histidine associated effect is described with a simple two-state model, where $\mathrm{KCVPBCV}_{-1} \mathrm{L94H}$ can pass from a state of high conductance (Gmax) to a state of low conductance ( $G \mathrm{~min}$ ) either via an effect which is inherent to the wild type protein or via an effect which is introduced by the mutation to histidine. Fitting the results based on this model shows that the effect after mutation of $\mathrm{L} 94 \mathrm{H}$ completely masks the effect that $\mathrm{H}^{+}$has on the wild type channel. Further, the effect during the transition from deprotonated to protonated histidine $(\mathrm{pH} 6) \mathrm{can}$ be mimicked, by addition of $\mathrm{NiCl}_{2}$ to the bath solution during measurements of $\mathrm{KCVPBCV}_{-1} \mathrm{L94H}$ at low $\mathrm{H}^{+}$ concentrations. Deprotonated histidine is known to coordinate $\mathrm{Ni}^{2+}$. However, the complexity of this $\mathrm{H}^{+}$ dependency does not allow to use this as a sensor system for $\mathrm{pH}$.

In the last part of the study two additional small viral $\mathrm{K}^{+}$channels, KCVGNLD and KCVMT325 Were examined in PLBs. So far both channels have only been characterized on a macroscopic level either by patch clamp measurements in HEK293 cells or with two-electrode voltage clamp measurements in oocytes. The two channels differ slightly in their aligned amino acid sequence but are quite similar in respect to conductance and open probability. The open probability is voltage-dependent, decreasing from $100 \%$ to $\sim 10 \%$ with positive voltages. With increasing $\mathrm{K}^{+}$concentrations the voltage-dependent decrease of the open probability is shifted towards more positive voltages. Also, KcVGNLD and KcVMT325 both possess a threonine in the selectivity filter directly before the GY/FG motive, compared to most other KcV channels which possess a valine at this position. Mutation of threonine to valine results in a loss of the voltage dependency in both, $\mathrm{KCV}_{\text {GNLD }}$ and $\mathrm{KCV}_{\mathrm{MT}} 325$, despite the similarity of the two amino acids.

The results of the different experiments demonstrate that for small viral $\mathrm{K}^{+}$channels modification of basic channel properties such as an inversion or loss of voltage dependency, alteration of unitary single channel conductance or open probability can easily be achieved by mutation of only a single amino acid. This feature makes viral $\mathrm{K}^{+}$channels particularly suitable modules for the construction of synthetic channels. With only little effort, great differences in the phenotype of single channel properties can be achieved. 


\section{Zusammenfassung}

Kaliumkanäle sind eine wichtige Gruppe von lonenkanälen, die wesentliche physiologische Funktionen erfüllen. Alle bekannten $\mathrm{K}^{+}$Kanäle haben eine ähnliche Architektur mit einer zentralen, leitenden Pore, die eine hohe Ähnlichkeit in allen bekannten Lebensformen, Eukaryonten, Archaeen, Bakterien und sogar Viren aufweist. Letztere erweisen sich als eine äußerst Interessante Gruppe, da die aus Viren isolierten $\mathrm{K}^{+}$ Kanäle auf ein absolutes Minimum reduziert sind und im Grunde das Porenmodul von allen $\mathrm{K}^{+}$Kanälen repräsentieren. Trotz ihrer reduzierten Größe besitzen sie alle wesentlichen charakteristischen Eigenschaften von größeren, komplexeren $\mathrm{K}^{+}$Kanälen, was sie zu einem idealen Modellsystem zur Untersuchung von Struktur/Funktionsbeziehungen macht, welche die Permeabilität von Ionen und das gating der Kanäle bestimmt.

In dieser Arbeit wurde die planare Lipid bilayer (PLB) Technik verwendet um kleine, virale $\mathrm{K}^{+}$Kanäle auf Einzelkanalebene elektrophysiologisch zu untersuchen. Mit diesem auf das Wesentliche reduzierte Messsystem ist eine schnelle und einfache Änderung der experimentellen Bedingungen, wie pH, Ionenkonzentration oder Lipidzusammensetzung, möglich, so wie das unkomplizierte Hinzugeben von Blockern. Im ersten Teil wird eine neve Methode zur Funktionsanalyse von Membranproteinen vorgestellt. Es konnte gezeigt werden, dass die Zugabe von nanodiscs zu einem in vitro Expressionssystem in einer zeitsparenden und kontaminationsfreien Methode resultiert, um Membranproteine zu exprimieren und aufzureinigen, damit sie anschließend mit verschiedenen Methoden für Einzelkanalmessungen verwendet werden können. Der virale $\mathrm{K}^{+}$Kanal KcvNis und der häufig als Modell verwendete, bakterielle $\mathrm{K}^{+}$Kanal KcsA konnten erfolgreich nach Expression in Anwesenheit von nanodics mit der PLB Methode untersucht werden. Die Experimente haben außerdem ergeben, dass nicht die Lipide innerhalb der nanodiscs die Kanaleigenschaften bestimmen, sondern die Lipidzusammensetzung der Zielmembran innerhalb des Versuchsaufbaus. Die Daten zeigen ebenfalls, dass diese Methode für den synthetischen, durch Blaulicht aktivierten $\mathrm{K}^{+}$Kanal BLINK 1 erfolgreich angewendet werden konnte. Im Gegensatz zur Expression in Zellen verliert BLINK1 jedoch seine Lichtempfindlichkeit, was ein Defizit der Methode hinsichtlich posttranslationaler Modifikationen darstellt.

Im zweiten Teil geht es um die systematische Analyse des viralen $\mathrm{K}^{+}$Kanals KCVPBCV-1. Eine Untereinheit besteht aus 94 Aminosäuren, welche zwei Transmembran (TM) Helices, eine Porenschleife mit dem Selektivitätsfilter und eine kurze N-terminale Helix ausbildet. Vorangehende Studien mit Hefekomplementationsassays haben ergeben, dass die Position 94 in KCVPBCV-1 eine wichtige Rolle beim gating spielt. In diesen vorherigen Versuchen wurde Leucin durch alle weiteren proteinogenen Aminosäuren ausgetauscht und das Maß der Hefekomplementation als indirekter Parameter für Kanalaktivität festgestellt. In dieser Arbeit wurde KCVPBCV-1 und die 19 Mutanten KCVPBCV-1 L94X auf Einzelkanalebene analysiert um den Effekt jeder einzelnen Mutation auf die wichtigsten Kanaleigenschaften, Einzelkanalleitfähigkeit und Offenwahrscheinlichkeit, zu überprüfen. Die Ergebnisse der PLB Messungen sind nicht kompatibel mit denen der Hefekomplementationsassays. Das bedeutet, dass letzteres eine geeignete Methode ist um die grundlegende Kanalfunktion zu überprüfen, jedoch wird keine Information über detaillierte Kanaleigenschaften geliefert. Die Ergebnisse der 
Hefekomplementationsassays sind vermutlich auch durch sekundäre Faktoren, wie Sortierung und Translationseffizient des Kanalproteins beeinflusst. Dennoch zeigen die Ergebnisse der Einzelkanalmessungen, dass die letzte Aminosäure von KCVPBCV-1 eine komplexe Auswirkung auf die Kanalfunktion hat und sowohl Einfluss auf die Offenwahrscheinlichkeit, als auch die Einzelkanalleitfähigkeit nimmt. Die wichtigsten Beobachtungen nach Messung der Mutanten waren, dass KcVPBCV-1 L94P und KCVPBCV-1 L94C ZU einer Erhöhung der durchschnittlichen Offenwahrscheinlichkeit, so wie der Spannungsabhängigkeit führen. Ein weiteres Ergebnis war, dass nach Mutation zu Aminosäuren mit basischen Seitenketten zum einen die Spannungsabhängigkeit der Offenwahrscheinlichkeit invertiert und zum anderen die Einzelkanalleitfähigkeit im Vergleich zum Wild Typ deutlich reduziert ist. Aufgrund der Titrierbarkeit von Histidin wurden sowohl KCVPBCV-1, also auch die Mutante KCVPBCV-1 L94H im pH Bereich zwischen 4 und 9 auf ihre $\mathrm{H}^{+}$Sensitivität untersucht. Dabei stellte sich heraus, dass bereits der Wild Typ eine $\mathrm{H}^{+}$Sensitivität aufweist, die durch Mutation von L94H deutlich verstärkt wird. Um den mit Histidin zusammenhängenden Effekt zu beschreiben wurde ein einfaches Zwei-Zustandsmodell angewendet, in welchem sich der Kanal in einem Zustand mit hoher Leitfähigkeit ( $G_{\max }$ ) oder niedriger Leitfähigkeit ( $G_{\min }$ ) befinden kann. Der Übergang von einem Zustand in den anderen kann über zwei Wege erfolgen, entweder durch den Effekt der mit dem Wild Typ einhergeht, oder durch den Effekt der durch die Mutation L94H ausgelöst wird. Werden die Daten basierend auf diesem Modell gefittet, ergibt sich, dass der Effekt durch die Mutation L94H den des Wild Typs komplett überschreibt. Außerdem konnte der Effekt der im Übergangsbereich von deprotoniertem zu protoniertem Histidin $(\mathrm{pH} 6)$ auftritt durch Zugabe von $\mathrm{NiCl}_{2}$ zur Elektrolytlösung während der Messung von KCVPBCV-1 $\mathrm{L94H}$ bei niedrigen $\mathrm{H}^{+}$Konzentrationen imitiert werden. $\mathrm{Ni}^{2+}$ ist dafür bekannt, mit deprotoniertem Histidin zu komplexieren. Die Komplexität dieser $\mathrm{H}^{+}$Abhängigkeit erlaubt es jedoch nicht daraus auf eine $\mathrm{pH}$ Sensorik zu schließen.

Im letzten Teil der Arbeit werden zwei weitere virale K+ Kanäle, KcVGnLD und KcVMT325 mit der PLB Methode untersucht. Bisher wurden die Kanäle erst anhand von patch clamp Messungen in HEK293 Zellen oder mit der two-electrode voltage clamp Technik in Xenopus Oozyten untersucht. Die beiden Kanäle unterscheiden sich zwar geringfügig in ihrer Aminosäuresequenz, zeigen aber sehr ähnliche Eigenschaften in Bezug auf Einzelkanalleitfähigkeit und Offenwahrscheinlichkeit. Die Offenwahrscheinlichkeit beider Kanäle ist spannungsabhängig und nimmt von nahezu 100\% auf 10\% ab mit höheren positiven Spannungen. Eine Erhöhung der $\mathrm{K}^{+}$Konzentration führt zu einer Verschiebung der Spannungsabhänigkeit in Richtung positiver Spannungen. Außerdem weisen beide Kanäle innerhalb des Selektivitätsfilters ein Threonin an der Position unmittelbar vor dem GY/FG Motiv auf, im Vergleich zu den Meisten anderen Kcv Kanälen, welche an dieser Position ein Valin besitzen. Die Substitution von Threonin zU Valin führt trotz der Ähnlichkeit der beiden Aminosäuren sowohl in KCVGNLD als auch in KcVMT325 ZU einem verlust der Spannungsabhängikeit.

Die Ergebnisse der verschiedenen Experimente zeigen, dass bei viralen $\mathrm{K}^{+}$Kanälen bereits durch einzelne Mutationen die grundlegenden Kanaleigenschaften wie Spannungsabhängigkeit, Offenwahrscheinlichkeit und Einzelkanalleitfähigkeit stark verändert werden können. Diese Eigenschaft macht virale $\mathrm{K}^{+}$Kanäle zu einem besonders geeigneten Baustein zur Herstellung synthetischer Kanäle. Mit nur geringem Aufwand können große Unterschiede auf der Einzelkanalebene erreicht werden. 


\section{Introduction}

\subsection{Membranes and membrane proteins}

To ensure compartmentalization, cells and cell organelles are surrounded by biological membranes. These membranes consist of a $\sim 5 \mathrm{~nm}$ thick lipid bilayer (Adam et al., 2009). They are composed of amphipathic fatty acids, which possess a long, hydrophobic hydrocarbon chain and a hydrophilic carboxyl head group. This property is the reason lipids spontaneously form bilayers when exposed to an aqueous environment (Adam et al., 2009). In mammalians, the major components of membranes are phospholipids and cholesterol (Spector \& Yorek, 1985). In phospholipids, the hydrophilic head consists of glycerol, phosphate, two fatty acids and an alcohol. The most frequently occurring phospholipids in biological membranes are phosphatidylcholine (PC, 45-55 mol\%), phosphatidylethanolamine (PE 15-25 mol\%), phosphatidylinositol (PI 10-15 mol\%) and phosphatidylserine (PS, 5-10 mol\%); another 15-20 mol\% consist of cholesterol, the most prominent member of the sterols (Stillwell, 2016). A regular lipid bilayer represents a barrier for dissolved substances (Deamer \& Bramhall, 1986). However, some of the functions of biological membranes is the maintenance of transmembrane gradients for e.g. ions and regulation of the resulting membrane potential, so ion transport across the membrane is mandatory (Adam et al., 2009). To enable the exchange of substances between the extra- and intracellular space, biological membranes additionally contain a large variety of peripheral, amphitropic and integral proteins (Stillwell, 2016). Together, lipids and proteins form a fluid, dynamic structure (Singer \& Nicolson, 1972). Peripheral proteins like cytochrome $c$ are globular, water-soluble proteins that are attached to the cellular membrane and interact with it through electrostatic, hydrophobic and selective fatty acid modification (Whited \& Johs, 2015; Stillwell, 2016). This interaction can be either permanent (peripheral) or transient (amphitropic). Amphitropic proteins like Src kinase or protein kinase $C$ can be either in the cytoplasm or recruited to the membrane when necessary. Switching between the locations is due to reversible conformational changes implemented by enzymes that catalyze hydrolysis, phosphorylation or dephosphorylation (Burn, 1988; Hurley, 2003). Integral membrane proteins (IMP) make up $20-30 \%$ of the genome (Gerstein \& Hegyi, 1998; Wallin \& Heijne, 1998). They are characterized by the fact that they partially or entirely span the membrane and are permanently bound to it. Partially integrated proteins are inserted into the membrane from either the cytosolic (endo protein) or extracellular side (ecto protein), while trans-membrane proteins span the entire membrane and are exposed to both, the cytosolic and the extracellular matrix (Stillwell, 2016). A typical example for partially and fully integrated membrane proteins are cytochrome $b_{5}$ and bacteriorhodopsin, respectively. While for investigation of membrane proteins peripheral and amphitropic proteins can easily be dissociated from membranes with a mild treatment, the majority of membrane proteins are IMPs. To isolate IMPs from the surrounding membrane it requires much more aggressive treatment which involves destroying of the membrane. Once isolated, IMPs are still often not completely free of lipids, are insoluble or aggregate in aqueous solutions (Singer \& Nicolson, 1972). For these reasons, the number of known membrane protein structures is insufficient, compared to soluble proteins (Ostermeier \& Michel, 1997; Shimizu et al., 2018). 
IMPs are structurally very diverse and involved in a variety of biological processes. One of the major physiological roles is related to the transport of molecules across membranes. To enable reactions inside isolated compartments, a selective transport of required substances across the membrane is necessary. To enable a controlled permeability, IMPs can be divided into transporters and ion channels (Adam ef al., 2009; Alberts et al., 2009). They differ in the fact that transporters have defined binding sites for distinct ions or small molecules, while ion channels are regulated through a number of gating mechanisms, allowing ions of a specific size and charge to pass (Alberts et al., 2009). Crossing of the membrane via transporters can occur through active or passive transport. Active transport requires energy and is necessary to transport ions or small molecules against their electrochemical gradient. Responsible proteins, e.g. pumps, gain the required energy through ATP hydrolysis (Lodish, 2003), light or redox processes (Läuger, 1985). For passive transport, ions or molecules are moved down their concentration gradient, so no energy is required (Alberts et al., 2009). Transporter proteins can also be referred to as carriers, which can be divided into three different types: uniporters, symporters and antiporters. Uniporters can transport a single small molecule down its concentration gradient (passive transport). Sym- and antiporters use the energy stored in the electrochemical gradient from a passive transport of an ion or small molecule to enable an active transport of one or more ions/small molecules simultaneously. This can occur either in the same (sym-) or opposite (antiporter) direction.

The majority of cellular processes, e.g. maintenance of the membrane potential, neuronal signal propagation, muscle innervation and synaptic transmission are regulated by ions. Since lipid bilayers are impermeable to ions, the membrane is equipped with a range of the above-mentioned IMPs, which enable the exchange of substances between the cytoplasm and the extracellular matrix. Due to the structure of transporters, transport rates are rather slow $\left(10^{\circ}\right.$ to $10^{4}$ ions/molecules per second; Lodish, 2003). For many cellular processes, a much faster response is essential. To enable this rapid exchange of ions across the membrane, there is another group of IMPs: ion channels. Ion channels form an aqueous pathway, allowing a selective and regulated passive transport of ions across the membrane (Hille, 2001). While transporters have defined binding sites to distinguish between ions or small molecules (Lodish, 2003), selectivity in ion channels is defined by charge and size of the ion of transport. They can either be selective for a specific charge (anions, cations) or even more selective for a specific ion (such as $\mathrm{K}^{+}, \mathrm{Na}^{+}, \mathrm{Ca}^{2+}, \mathrm{Cl}^{-}$ , F-; (Hille, 2001)). Ions can only pass through the pore when the channel is in its open state. Switching from the open (conductive) to the closed (non-conductive) state is called gating. Due to the dynamic structure of ion channels, gating is a stochastic process that can be regulated by a variety of stimuli, such as pH, ligands, voltage, light or mechanical stress (Krol \& Trebacz, 2000; Hille, 2001b). Once ion channels are in an open state, they are able to catalyze a rapid ion flux across the membrane along their electrochemical gradient, reaching a characteristically high diffusion rate of up to $10^{8}$ ions per second, which is close to the diffusion rate of ions in water (Hille, 2001). It is highly desired to investigate and fully understand the structure/function relations of ion channels, since a large number of diseases concerning e.g. the cardiac and nervous system (so-called channelopathies) are associated with only single mutations within gene sequences that code for ion channels (Ashcroft, 2006). 


\subsection{Potassium channels}

As mentioned before, ion channels can be selective for a certain ion. In this case it favors the conductance of this particular ion strongly over other ions with similar charge and size. If the favored conductive ion is $\mathrm{K}^{+}$, the corresponding ion channels are named potassium channels ( $\mathrm{K}^{+}$channels). All known $\mathrm{K}^{+}$channels belong to a single protein family and can be found in both plasma membranes of every known form of life, eukaryotes, archaea, bacteria and even in viruses (Plugge et al., 2000; Mackinnon, 2003), as well as in membranes of cell organelles such as mitochondria, chloroplasts and the endoplasmic reticula (Thiel et al., 2013). $\mathrm{K}^{+}$channels contribute to a wide range of functions, e.g. termination of the action potential, maintenance of resting potential, release of hormones and transmitters or regulation of cell volume (Hille, 2001). Due to the great variability of functions, a highly precise regulation of $\mathrm{K}^{+}$channels is mandatory. In animals, $\mathrm{K}^{+}$channels can be divided into three main groups: voltage-gated $\mathrm{K}^{+}(\mathrm{Kv})$ channels, inward rectifying $\mathrm{K}^{+}$channels $\mathrm{K}^{+}(\mathrm{Kir})$ channels and two-poredomain $\mathrm{K}^{+}(\mathrm{K} 2 \mathrm{P})$ channels (Buckingham et al., 2005). The commonality of all $\mathrm{K}^{+}$channels is that four poreforming subunits assemble as a homodimer or -tetramer, to create a functional channel with a water filled pore in the center, composed of a gate, the selectivity filter (SF) and the cavity (figure (fig.) 2; Hille, 2001; Choe, 2002). What varies in $\mathrm{K}^{+}$channels are the different numbers of additional TM domains (TMD). While Kir channels have no additional TMDs and only consist of the pore module (2 TMDs), Kv channels have in total six TMDs. Kv and Kir channels assemble as tetramers to form a functional channel. K2P channels have the distinctive feature of possessing two pore-forming domains within the same subunit, so these channels assemble as dimers. In total K2P channels have four TMDs. One known exception is TOK-1, a K2P channe from Saccharomyces cerevisiae. It is the only known channel to possess eight TMDs (fig. 1, Buckingham et al., 2005; G. Thiel et al., 2013).
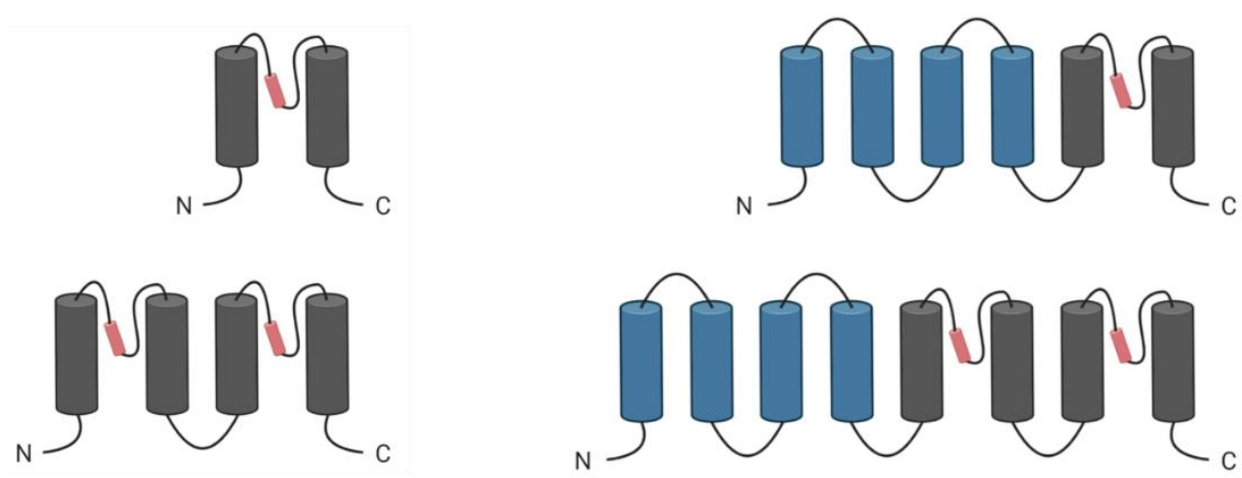

Figure 1- Schematic illustration of the amount of TMDs in different subunits of $\mathrm{K}^{+}$channels. All $\mathrm{K}^{+}$channels are composed of a pore module (top left), which consists of two TMDs (grey), which are connected by a pore loop, which contains the pore helix (red). The number of TMDs per subunit varies between 2, 4, 6 and 8. Additional subunits are represented in blue. $\mathrm{K}^{+}$channels with 2 and 6 subunits (top row) assemble as homotetramers to form a functional channel. K2P channels are characterized by containing two pore forming units within the same subunit (bottom row). Subunits consist of 4 or 8 TMDs and functional channels assemble as homodimers. 
A

B
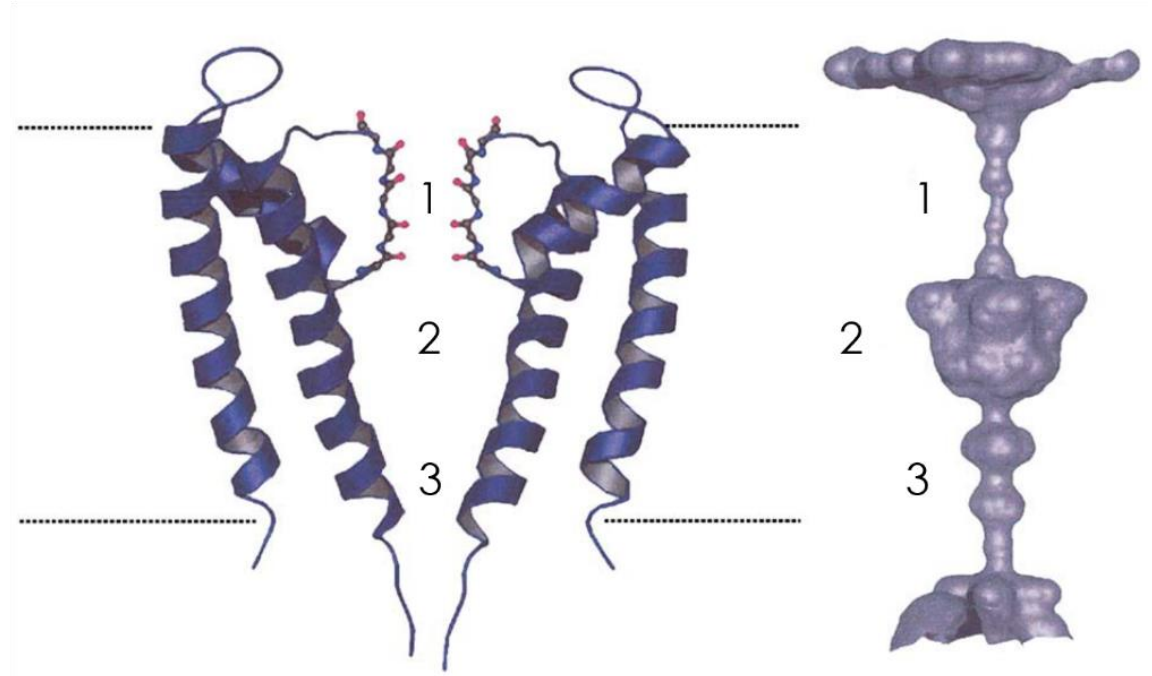

Figure 2 - Structure of KcsA and its pore. (A) Side view of two of the four subunits of KcsA. Dotted lines indicate the lipid bilayer. (B) The pore-lining surface of KCSA. SF (1), Cavity (2) and intracellular gate (3) are indicated with the respective numbers in the corresponding area. Modified after Sansom et al., 2002.

All functional $\mathrm{K}^{+}$channels are composed of a pore forming domain and a regulatory domain. While the pore-forming domain is responsible for $\mathrm{K}^{+}$transportation and is very similar throughout all $\mathrm{K}^{+}$channels, the regulatory domain detects numerous stimuli and is what distinguishes the channels from each other (Kuang et al., 2015). The essential ability of $\mathrm{K}^{+}$channels to have such a high selectivity for $\mathrm{K}^{+}$over other ions is enabled by the SF at the extracellular entrance of the pore. Permeability for $\mathrm{K}^{+}$can be as much as 1000 times higher than for $\mathrm{Na}^{+}$, even though the radius of the $\mathrm{K}^{+}$ion is with $1.33 \AA$ only slightly larger than $\mathrm{Na}^{+}$with $0.95 \AA$ (Doyle et al., 1998). Sequence alignment of a great variety of $\mathrm{K}^{+}$channels from all major classes revealed a highly homologous sequence of eight amino acids, TXXTXGY/FG, referred to as the signature sequence within the SF (Heginbotham et al., 1994). Within the pore, the SF forms the narrowest part. In aqueous solution, $\mathrm{K}^{+}$ions are fully hydrated, which increases their size over the radius of the SF.

To pass the SF, $\mathrm{K}^{+}$must strip off its hydration shell. Obtaining the first crystal structure of a $\mathrm{K}^{+}$channel from Streptomyces lividans (KCSA) helped understanding the mechanism of selectivity (Doyle et al., 1998). Since the process of dehydrating a $\mathrm{K}^{+}$ion requires a high energetic input, the carbonyl oxygens (in the case of threonine: the oxygen of the side chain) of the amino acids within the signature sequence of the SF are designed to imitate the ions hydration shell. The radius created between the carbonyl/side chain oxygens correlates exactly with the diameter of a single $\mathrm{K}^{+}$ion, reducing the energy barrier of dehydration for only this specific ion. At the entrance to the pore the first oxygen ring is formed by the second glycine of the GY/FG motif. Due to negatively charged amino acids positioned at the pore entrance, the local $\mathrm{K}^{+}$concentration is slightly increased, to attract $\mathrm{K}^{+}$ions. Within the SF are four $\mathrm{K}^{+}$binding sites (S1-S4), created by four layers of carbonyl oxygens from the remaining amino acids of the signature sequence. $\mathrm{K}^{+}$ions are positioned in the middle of two such layers, where four oxygens from each layer replace the eight water molecules of the hydration shell of $\mathrm{K}^{+} . \mathrm{K}^{+}$ions can bind within these four positions in two different conformations: $\mathbf{S 1}$ and $\mathrm{S} 3$, or $\mathrm{S} 2$ and $\mathrm{S4}$. In both cases, a water molecule is positioned between 
them (fig. 3). Depending on the electrochemical gradient, $\mathrm{K}^{+}$ions enter the SF from either side. The electrostatic repulsion between the individual $\mathrm{K}^{+}$ions shift the ions into the next configuration, releasing an ion on the other side of the SF, respectively. The SF opens to form a water-filled cavity, to deplete the charge of $\mathrm{K}^{+}$ions after exiting the $\mathrm{S} 4$ binding site. The precise arrangement of the amino acids of the SF that has the exact diameter of a $\mathrm{K}^{+}$ion allows the high selectivity for $\mathrm{K}^{+}$over $\mathrm{Na}^{+}$. Ions with a more similar radius to $\mathrm{K}^{+}$, like $\mathrm{Rb}^{+}(1.48 \AA)$ or $\mathrm{Cs}^{+}(1.69 \AA)$, can permeate through the pore with diffusion rates similar to $\mathrm{K}^{+}$(Clapham, 1998; Doyle et al., 1998; Zhou et al., 2001; MacKinnon, 2003; Lockless et al., 2007).

\subsection{Gating}

As mentioned before, ion channels must be in an open state for ion conduction. The controlled opening and closing of channels in response to various stimuli is called gating (Jiang et al., 2002). Depending on the channel, gating is regulated through a number of stimuli, such as changes in the membrane potential (Kv channels) or various chemical stimuli (Jiang et al., 2002; Ben-Abu et al., 2009). In the nervous system, communication between neurons and certain muscle cells is provided by electrical impulses, so-called action potentials (AP). APs are alterations of the electric potential across the membrane of the cells, triggering a variety of cellular responses at their destination, e.g. the release of neurotransmitters. The generation and conductance of APs, as well as excitability of these cells are the result of different gating properties of i.a. Kv channels (Hodgkin et al., 1952; Jiang et al., 2003; Lodish, 2003). In this context, three stages in which $\mathrm{K}^{+}$channels exist can be defined: closed, activated and inactivated (Kuang et al., 2015). In the case of Kv channels, the stimulus responsible for the transition from one state to the other is a change in the membrane potential. As mentioned, each subunit of $\mathrm{Kv}$ channels is composed of six TMDs, which assemble as a functional tetramer. Individual TMDs are named S1-S6. S5 \& S6 form the pore, which is responsible for ion conduction, while S1-S4 compose the voltage-sensing domain (VSD) (Jiang et al., 2003). Within each $\$ 4$ segment are several conserved arginine or lysine, which react to changes in the membrane potential by movement of the $\$ 4$ segment along the membrane (Aggarwal \& MacKinnon, 1996; Labro \& Snyders, 2012). This movement is coupled to the pore module through a linker helix between S4 and S5, resulting in opening of the intracellular activation gate after depolarization (Armstrong \& Bezanilla, 1974; Long et al., 2005; Jensen et al., 2012; Kuang et al., 2015).

Comparison of the crystal structures of the bacterial channel KcsA (Doyle et al., 1998) and the $\mathrm{Ca}^{2+}$ gated $\mathrm{K}^{+}$channel from Methanobacterium thermoautotrophicum (MthK) provided the first insight to the structure and conformational changes of the two different gates of $\mathrm{KV}$ channels (Jiang et al., 2002; MacKinnon, 2003). Conformational changes of these two gates characterize the different states of the channel (resting, activated, inactivated). The activation gate is located between the SF and the cytoplasm, composed of the inner TM helices. In the closed state they are bent, creating a barrier due to intersection of the lower parts of the helices at the intracellular side. This conformation is referred to as bundle-crossing (fig. 3). In the closed state, the diameter of the intracellular entrance of the bundlecrossing is only $3.5 \AA$, which is less than the radius of a hydrated $\mathrm{K}^{+}$ion, preventing the entry or exit of $\mathrm{K}^{+}$ ions from the cavity. The second gate includes conformational changes of the SF and is located at the extracellular side (Doyle et al., 1998; Perozo et al., 1999; Imai et al., 2010). In the resting state, the 
intracellular activation gate (bundle-crossing) is closed, while the extracellular gate (SF) is open (Doyle et al., 1998; Imai et al., 2010). Upon the receipt of an activating stimulus (voltage in the case of Kv channels, $\mathrm{pH}$ in the case of $\mathrm{KcSA}$ ), the conformation changes and the channel switches into the activated state. The narrow entrance formed by the bundle-crossing is widened, allowing $\mathrm{K}^{+}$ions to enter the cavity and to cross the membrane through the open SF. The responsible hinge that allows the conformational change between the resting and active state was located as a glycine residue, which is conserved in $\mathrm{K}^{+}$ channels from all different types of organisms, within the inner helix. Another conserved position is five amino acids towards the C-terminus from the glycine hinge: all aligned channels possess an amino acid with a small side chain, either glycine or alanine, which ensures a wide enough pathway for $\mathrm{K}^{+}$ions when the channel is in its activated state (Jiang et al., 2002; MacKinnon, 2003; Labro \& Snyders, 2012).
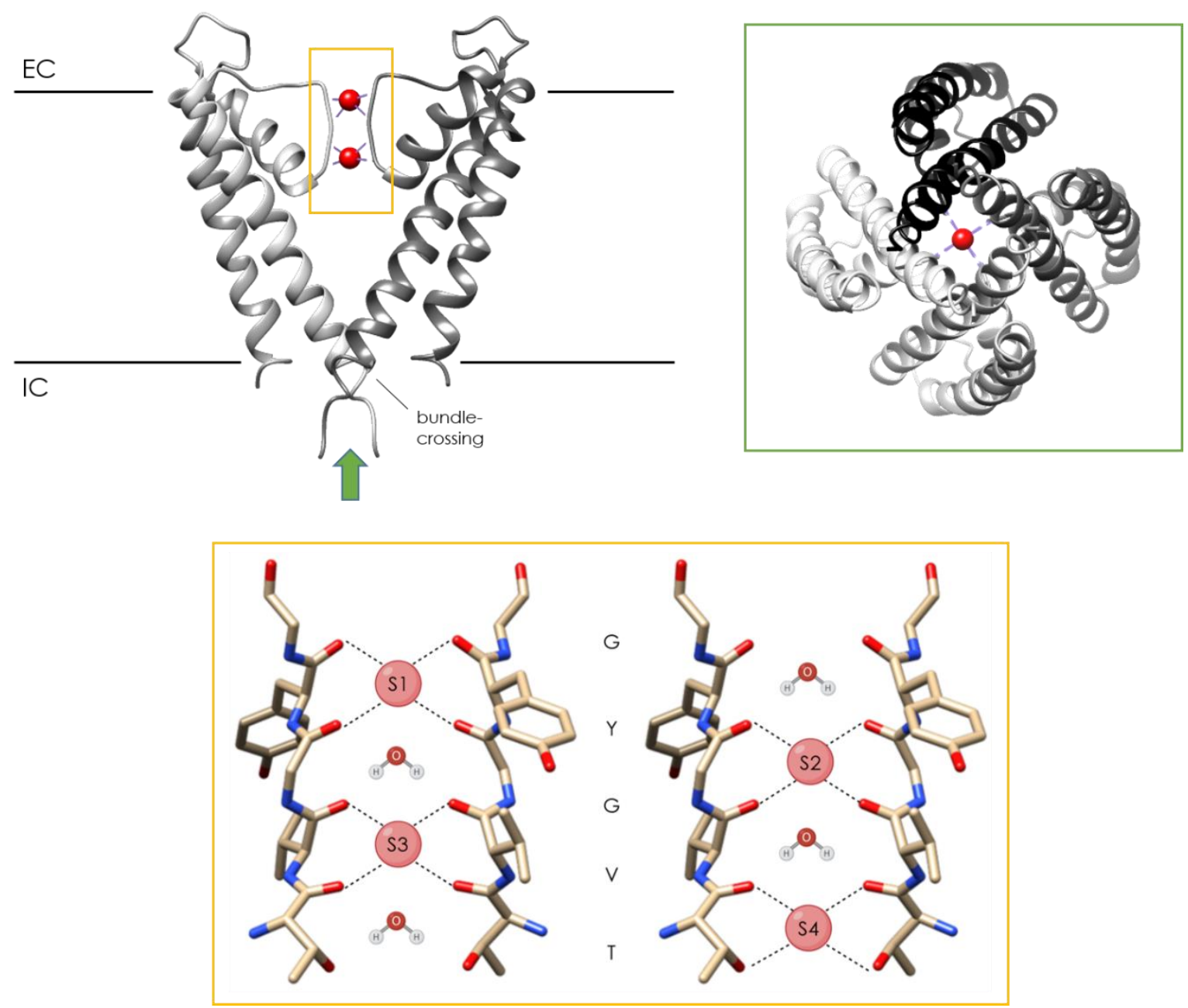

Figure 3 - Different views on the structure of the bacterial channel KcsA. Two of the four subunits are sown on the top left, with 2 of 4 possible ions bound within the SF. The channel is in its closed conformation, the ends of TM2 are intersected at the so-called "bundle crossing", blocking the entrance or exit for hydrated $\mathrm{K}^{+}$ions. Black lines indicate the lipid membrane, EC/IC stand for extracellular/intracellular space, respectively. In the green box is a bottom view of the channel, through the narrow opening of the bundle-crossing. The corresponding point of view is indicated with a green arrow. In the yellow box (bottom) is an enlarged and detailed view of the SF, with $\mathrm{K}^{+}$ ions (pink spheres) bound in the two 1,3 conformation (left) and 2,4 conformation (right). The respective $\mathrm{K}^{+}$free binding site has a water molecule bound, respectively. (PDB: 1K4C) 
Activation of $\mathrm{K}^{+}$channels is followed by inactivation. There are two different mechanisms for the inactivation of $\mathrm{K}^{+}$channels: $\mathrm{N}$-type and C-type inactivation (fig. 4; Kuang et al., 2015). N-type inactivation is a fast, voltage-independent process. Due to the responsible structure, it is often referred to as "ball and chain" mechanism. In Shaker-type $\mathrm{K}^{+}$channels, the inactivation gate is composed of the first 20 amino acids at the intracellular, N-terminal end of the channel. Shaker is a well-studied Kv channel from Drosophila melanogaster (Kuang et al., 2015). Within these 20 amino acids the first 10 amino acids are hydrophobic, representing the "ball", while the other 10 are hydrophilic, representing the "chain". While this inactivation gate is located at the end of each of the four subunits, only one gate is necessary for inactivation. Directly after activation, the hydrophobic "ball" of the N-terminus enters the cavity and acts like a plug - blocking the entry for further $\mathrm{K}^{+}$ions, while both the SF and the intracellular activation gate are still open (Hoshi et al., 1990; Kurata \& Fedida, 2006). The first three amino acids bind within the cavity, while the rest of the hydrophobic chain extends to the cytoplasmic entrance of the channel, followed by the hydrophilic area, which interacts with the aqueous protein surface (M. Zhou et al., 2001). Due to the structural similarities of the N-terminal end of this gate to quaternary ammonium (QA) ions, a commonly known property of $\mathrm{K}^{+}$channels is the ability to be blocked by QA ions, such as TEA (Choi et al., 1991; Kurata \& Fedida, 2006). The binding of the ball peptide is voltage-dependent, at negative voltages it is released back into the cytoplasm, reopening the channel (Ruppersberg et al., 1991).

A second, usually slower type of inactivation was identified, known as C-type inactivation. In contrast to $\mathrm{N}$-type inactivation, it is independent of a structure located at the C-terminus (Hoshi et al., 1991). The mechanism behind C-type inactivation is still poorly understood. However, it is known to be associated with conformational changes of the area of the external mouth, as well as the SF (Cuello et al., 2010; McCoy \& Nimigean, 2012; Hoshi \& Armstrong, 2013; Kuang et al., 2015). A molecular mechanism is suggested by Cuello et al. (2010), where the rearrangements of the SF are coupled to the opening of the bundle crossing in KCSA. Two conformations of the SF are proposed, in which first the S2, and later S3 binding site for $\mathrm{K}^{+}$becomes increasingly destabilized. With only S1 and S4 left as functional binding sites, the SF collapses and is no longer able to conduct $\mathrm{K}^{+}$ions. Imai et al. (2010) have also described the activation-coupled inactivation of KcsA, which occurs upon switching from neutral $(\mathrm{pH} 7)$ to acidic $(\mathrm{pH}$ 4) conditions in a similar way. At $\mathrm{pH} 7$ the intracellular inactivation gate is closed. Increasing the $\mathrm{H}^{+}$ concentration triggers the opening of the gate, accompanied by a high peak current in electrophysiological measurements. Immediately afterwards a slow reduction of the current due to the slow process of inactivation can be seen. It is induced by the loss of $\mathrm{K}^{+}$affinity of KcsA upon conformational change of the SF after activation. In the inactivated state, no $\mathrm{K}^{+}$is bound, but $\mathrm{H}_{2} \mathrm{O}$. While for $\mathrm{N}$-type inactivation only one of the $\mathrm{N}$-terminal structures is necessary for inactivation, the four subunits interact cooperatively during C-type inactivation (Ogielska et al., 1995; Panyi et al., 1995). It is also known that both, an increase of monovalent cations, as well as addition of extracellular TEA slow down the rate of C-type inactivation (López-Barneo et al., 1993; Kiss et al., 1999; McCoy \& Nimigean, 2012). 


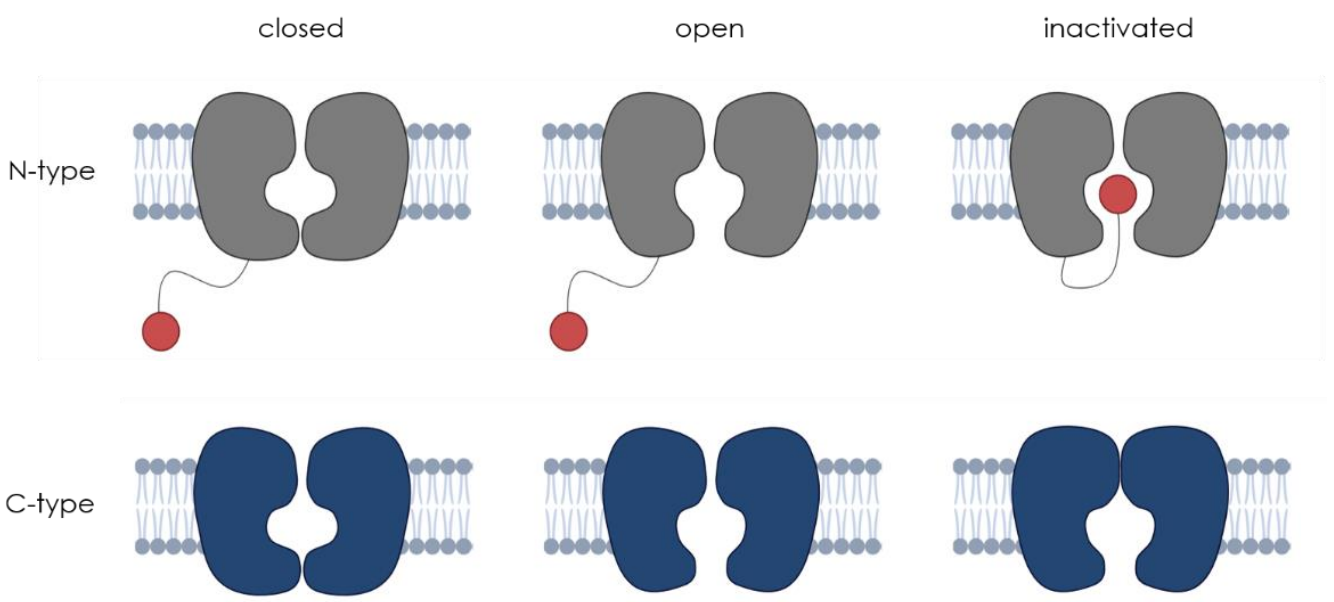

Figure 4 - Schematic illustration of the different types of inactivation. Three different stages (closed, open and inactivated) for $\mathrm{N}$-type (top, grey) and C-type (bottom, blue) inactivation. During the fast $\mathrm{N}$ type inactivation, a small peptide (red ball) binds within the cavity, while the intracellular gate is still open. During C-type inactivation the intracellular gate is also still open, but the SF undergoes conformational changes, preventing the SF from conducting $\mathrm{K}^{+}$ions.

\section{$1.4 \quad$ Viral K+ channels}

It has been known for some time that even the smallest forms of organic structures, viruses, encode for functional ion channels. These include some approximately 100 amino acid long membrane proteins from influenza A, B and C, or HIV-1, which form as oligomers and enable ion flux (Fischer \& Sansom, 2002), as well as viroporins, which are 60-120 amino acids in size, interacting with the membrane to modify the permeability of host cells for ions (Gonzalez \& Carrasco, 2003). Viral encoded $\mathrm{K}^{+}$channels represent another interesting group of ion channels. Sequencing of the 330-kb double-stranded genome of the Paramecium bursaria chlorella virus (PBCV-1) revealed a 94 amino acid sequence, which turned out to be the first virus-encoded functional $\mathrm{K}^{+}$channel, known as KCVPBCV-1 ( $\mathrm{K}^{+}$(channel) chlorella virus Paramecium bursaria chlorella virus 1; Plugge et al., 2000; Van Etten, 2003). Like K2P channels, KCVPBCV-1 only consists of 2 TMDs, with the difference of being reduced to an absolute minimum and therefore representing the basic pore modules of $\mathrm{K}^{+}$channels. Despite its minimalistic size, it still possesses all the features which are characteristic for bigger and more complex $\mathrm{K}^{+}$channels (Plugge et al., 2000; Van Etten, 2003).

The structure of KCVPBCV-1 is composed of two TM helices (TM1 and TM2), which are connected by 44 amino acids. The pore is formed within these 44 amino acids, where also the characteristic $\mathrm{K}^{+}$channel signature sequence THSTVGFG is located. While there is a short N-terminus of 12 amino acids in length, $\mathrm{KCV}_{\mathrm{PBCV}-1}$ has no cytosolic C-terminal domain: the COOH-terminus is integrated at the end of TM2 (fig. 5; Plugge et al., 2000; Tayefeh et al., 2007). Expression of KCVPBCV-1 in different heterologous systems, such as Xenopus oocytes (Gazzarrini et al., 2002; Plugge et al., 2000), human embryonic kidney (HEK) 293 cells (Moroni et al., 2002), CHO cells (Gazzarrini et al., 2003) or yeast (Gebhardt et al., 2012; Hamacher et al., 2012) has shown that not only structurally, but also functionally KCVPBCV-1 shows all the basic properties of $\mathrm{K}^{+}$channels (Plugge et al., 2000; Gazzarrini et al., 2003; Thiel et al., 2013). These include a higher selectivity 
for $\mathrm{K}^{+}$over $\mathrm{Na}^{+}$ions (permeability ratio $\mathrm{P}_{\mathrm{K}} / \mathrm{P}_{\mathrm{Na}}=$ 9.32, Plugge et al., 2000) and a block of the inward current after addition of external $\mathrm{Ba}^{2+}$ (Plugge et al., 2000). KCVPBCV-1 is also sensitive to the antiviral drug amantadine, which is known to inhibit the influenza virus M2 at concentrations below $1 \mu$ M (Wang et al., 1993; Plugge et al., 2000). Despite the basic layout and the lack of a big N-terminus or any C-terminus, macroscopic currents in Xenopus oocytes were composed of two components: an instantaneous and a time-dependent component (Plugge et al., 2000; Gazzarrini et al., 2002). In whole-cell measurements in HEK293 (Moroni et al., 2002) and CHO (Gazzarrini et al., 2003) cells these two components could also be observed, although the time-dependent component was only visible in cells which had a high expression level. Another unusual property of the observed currents is a slight voltage-dependency. KCVPBCV-1 lacks any obvious voltage-sensing domain, which are present in KV channels in form of the S4 VSD. An obvious assumption was that the short 12 amino acid $\mathrm{N}$-terminus might be responsible for voltage sensing, due to two positively charged amino acids within the sequence. Neutralizing the charges in the $\mathrm{N}$-terminus had however no effect on the voltage-dependency (Gazzarrini et al., 2002). Truncation of the entire Nterminus in contrast leads to a loss of channel function (Gazzarrini et al., 2002; Moroni et al., 2002).
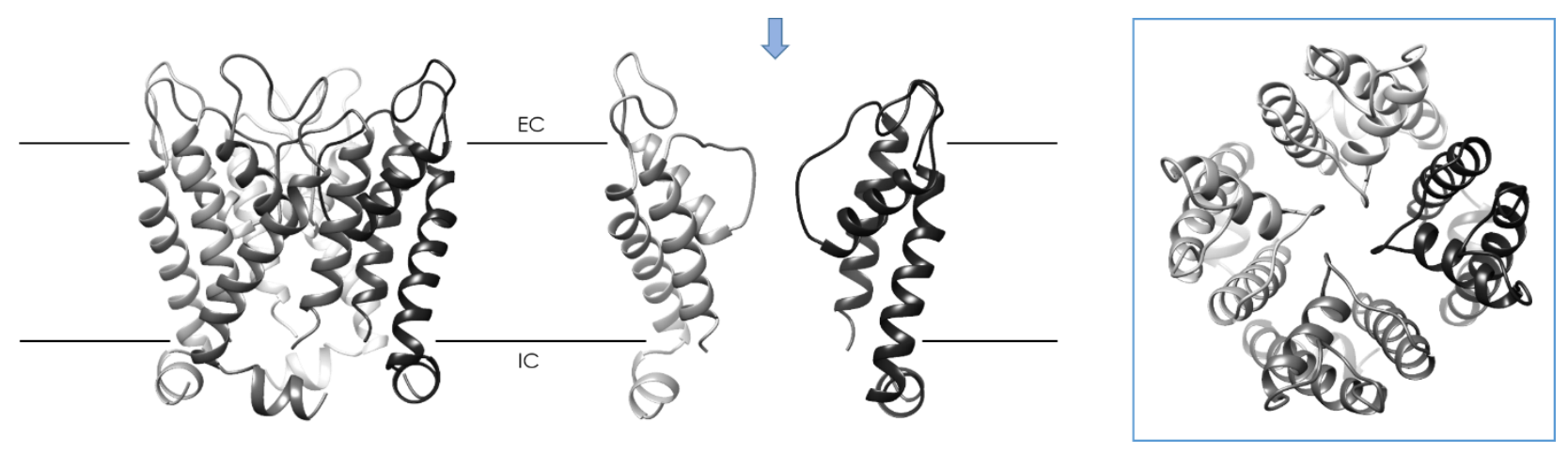

Figure 5 - Homology model of the viral $\mathrm{K}^{+}$channel KCVPBCV-1. KCVPBCV-1 is shown as a tetramer (left). Each subunit is shown in a different shade of grey. Only two opposite Subunits are shown in the middle. The black lines indicate the lipid membrane, EC/IC mark the extracellular and intracellular space, respectively. A top view is shown on the right, outlined with a blue box. The point of view is indicated with a corresponding blue arrow. Homology model created by Hoffgaard et al., 2015; Tayefeh et al., 2009.

KCVPBCV-1 Could also be successfully reconstituted into planar lipid bilayers (PLB, Pagliuca et al., 2007; Shim et al., 2007) for single channel measurements. The protein was expressed either in Pichia pastoris, with subsequent reconstitution into liposomes (Pagliuca et al., 2007) or expressed in vitro (Shim et al., 2007). In both cases, the same channel characteristics could be observed on the single channel level as previously in macroscopic measurements. KCVPBCV-1 showed a strong permeation preference for $\mathrm{K}^{+}$over $\mathrm{Na}^{+}$and could be blocked by external $\mathrm{Ba}^{2+}$ and amantadine. Interestingly, the addition of $\mathrm{Ba}^{2+}$ had no effect on the conductance of the channel, but strongly reduced the mean dwell time of the open events (which results in a reduction of the open probability). Amantadine had the opposite effect: the mean dwell times of the open events were unaffected, but there was a $53 \%$ drop of the unitary single channel conductance, implying that the blocking effects for $\mathrm{Ba}^{2+}$ and amantadine are attributed to two different mechanisms (Shim et al., 2007). 
The corresponding I/V curve of KCVPBCV-1 shows a linear progression between high positive voltages until - -60 mV, followed by a negative slope conductance. This phenomenon has been identified as an apparent effect from the measuring device. It is the result of a very fast gating process of the channel at high negative membrane potentials, known as flicker gating. Individual opening and closing events are too fast to be fully resolved by the device, which reflects as an increase of noise for the open level accompanied by an apparent decrease of conductance. The true current could be deducted from beta fit analysis and was shown to follow the linear extrapolation of the ohmic conductance seen in the range (Abenavoli et al., 2009; Schroeder, 2015; Schroeder \& Hansen, 2006, 2007).

Though no obvious sensing domains are found in $\mathrm{KCVPBCV}_{-1}$, it still shows distinct gating properties, such as the voltage dependency, activation upon hyperpolarization and a slow time-dependent inactivation (Plugge et al., 2000) in macroscopic and single channel measurements. Investigation of the inner TM helix has also revealed that it is too short for the typical bundle-crossing seen in KcsA and other gated $\mathrm{K}^{+}$ channels (Gazzarrini et al., 2003). The conserved glycine in the inner TM of non-viral $\mathrm{K}^{+}$channels which serves as a hinge for opening and closing of the intracellular activation gate (Jiang et al., 2002; MacKinnon, 2003; Labro \& Snyders, 2012) is also absent in KCVPBCV-1, confirming the absence of the bundlecrossing. Previous studies have identified two possible gates in KCVPBCV-1. One is located within the SF and is also responsible for flicker gating (Abenavoli et al., 2009). The other one is located at the C-terminus and is a result of constant formation and disruption of salt bridges, which are formed between the free negative charge at the C-terminal end of the protein and positively charged amino acids within the 12 amino acid long N-terminal segment (Hertel et al., 2010; Moroni et al., 2002). While the salt bridge is formed, the channel is open. Disruption of the salt bridge leads to exposure of the four negatively charged ends of the C-terminus, which subsequently bind $\mathrm{K}^{+}$ions and block the entrance to the cavity for further ions to exit or enter (Baumeister, 2010; Hertel et al., 2010).

Meanwhile, a wide range of small viral $\mathrm{K}^{+}$channels have been identified, besides KCVPBCV-1. Over 40 functional $\mathrm{K}^{+}$channels could be found in other viruses that infect the same, or similar hosts as PBCV-1, the algae Chlorella NC64A (Thiel et al., 2011). Another channel that is well-characterized is KCVATCV-1, from Acanthocystis turfacea. With only 82 amino acids per subunit, it is even smaller than $K_{C V} V_{P B V}-1$ and even further reduced, completely lacking any N-Terminus. Despite the even smaller size, like KCVPBCV-1 it possesses the same major features as other $\mathrm{K}^{+}$channels, such as strong $\mathrm{K}^{+}$selectivity over $\mathrm{Na}^{+}$and sensibility towards typical K+ channel blockers like Ba ${ }^{2+}$ (Gazzarrini et al., 2009). Although both proteins share a considerable amount of sequence identity or similarity (fig. 6), the single channel properties are quite different. While the open probability for KCVPBCV-1 is very low (below 30\%, Pagliuca et al., 2007), for $\mathrm{KCV}_{\mathrm{ATCV}-1}$ it is very high (Gazzarrini et al., 2009). On the other hand, KCVATCV-1 has a much lower unitary

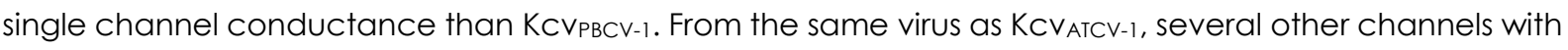
similar size and a high sequence similarity have been isolated (Fitzgerald et al., 2007), referred to as ATCV1 like channels (fig. 6). Despite the high sequence similarity, differences in gating can still be recognized.

The great diversity within these minimal Kcv channels, while still exhibiting all the characteristic hallmarks of bigger and more complex $\mathrm{K}^{+}$channels makes them such an interesting model system to analyze and 
characterize basic structure/function relations (Thiel et al., 2011). This information hopefully can be transferred to more complex systems to help fully understand underlying mechanisms of channel gating or to design and build synthetic channels with new functional properties.

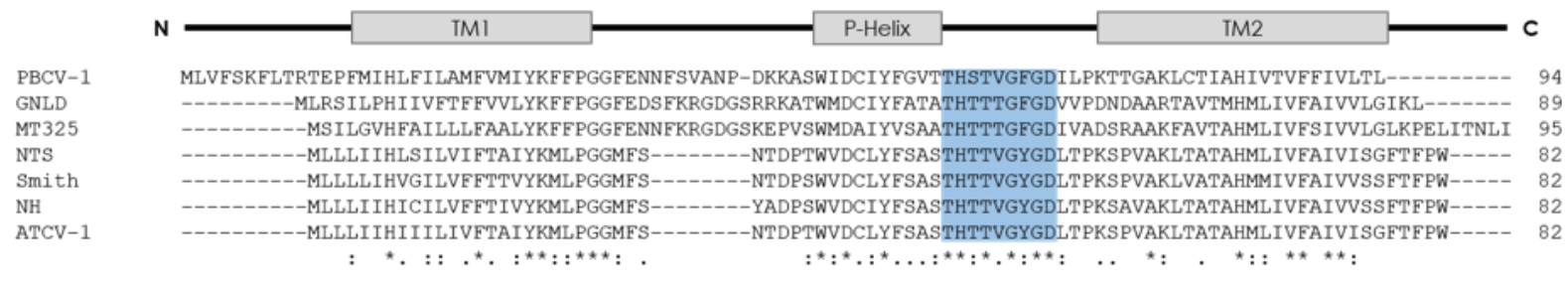

Figure 6 - Sequence alignment of a selection of Kcv channels. Listing of a choice of KcV channels encoded by different Chlorella viruses (Name of the corresponding channels on the left, length of the amino acid sequence listed on the right). The grey boxes above the sequence indicate the first, outer TM helix (TM1), the pore helix (P-Helix) and the second, inner TM helix (TM2). The signature sequence of the selectivity filter is highlighted in blue. Among the sequences, identical amino acids are marked by stars, conservative and semi-conservative amino acid differences are marked by colons or dots, respectively. The alignment was created with Clustal Omega (Madeira et al., 2019).

In this study, viral $\mathrm{K}^{+}$channels are used as model systems to further analyze and understand the underlying mechanisms that are responsible for gating in such minimalistic structures. The first part is dedicated to a new method for functional analysis of $\mathrm{K}^{+}$channels. By combining commercially available nanodiscs with an in vitro expression system, we established a reliable method for protein expression. Not only is this method extremely timesaving, it also provides a high yield of protein, without exhibiting any contaminations. From the start of protein expression to the end of purification only takes a few hours and measurements can take place the same day. Additionally, we could show that not the lipids contained in the nanodiscs determine channel function, but the target membrane used in the experimental setup.

The next part of the study addresses the C-terminus of KCVPBCV-1. As mentioned above, one of the gates is located at the intracellular side and is constructed by constant formation and disruption of salt bridges between the free negative charge of the C-terminus and the positively charged amino acids within the $\mathrm{N}$-terminus of the channel. Previous studies with yeast complementation assays (Gebhardt, 2010) have shown that position 94 plays an additional role besides provision of a free negative charge. To analyze the importance of the side chain at this position, leucine 94 was mutated to all other 19 proteinogenic amino acids and analyzed on the single channel level for planar lipid bilayer (PLB) measurements. The results should also show to what extend the results of the two methods (yeast complementation assays and PLB) are comparable.

In the last part of the study two relatively new small viral $\mathrm{K}^{+}$channels, KCVMT325 and KCVGNLD are examined more closely. So far both channels have only been characterized either by patch clamp measurements (Siotto, 2017) or two-electrode voltage clamp measurements in Xenopus oocytes (Gazzarrini et al., 2006). Here, both channels were characterized on the single channel level with the PLB method with different $\mathrm{K}^{+}$concentrations. Further, an alignment of numerous viral $\mathrm{K}^{+}$channels shows that the primary amino acid within the signature sequence directly before the GY/FG motive is valine. Interestingly, KCVMT325 and 
KCVGNLD both possess a threonine at this position. To examine the relevance of this position, threonine was mutated to valine in both channels. Hopefully, the provided information will help better understand the links between different amino acids and the resulting gating mechanisms of small viral $\mathrm{K}^{+}$channels. 


\section{Materials and methods}

\subsection{Molecular biological methods}

\subsubsection{Site-directed mutagenesis PCR}

The coding sequence of $\mathrm{KcV}$ has previously been cloned into the expression vector PEXP5-CT/TOPO using the PEXP-5-CT/OPOTM TA Expression Kit (Thermo Fisher Scientific, Waltham, MA, USA), following the manufacturer's instructions. All KcvL94X mutants were introduced via site-directed mutagenesis (SDM) polymerase chain reaction (PCR) (Ho et al. 1989; Papworth 1996), using the Phusion-DNA-Polymerase (Thermo Fisher Scientific, Waltham, MA, USA) according to manufacturer's specifications. PCR products were electrophoretically separated in a $1.5 \%$ agarose gel in 1X TAE and purified using the Zymoclean ${ }^{\text {TM }}$ Gel DNA Recovery Kit (Zymo Research Europe GmbH, Freiburg, Germany) for gel extraction. 400 ng of the extracted DNA was added to $50 \mu \mathrm{l}$ chemically competent DH5 $\alpha$ cells and incubated on ice for 20-30 min. For transformation, a heat shock was performed for $45-60 \mathrm{~s}$ at $42^{\circ} \mathrm{C}$ with a subsequent, immediate transfer on ice for $2 \mathrm{~min}$. Afterwards $250 \mu \mathrm{l}$ of SOC Medium (20 g/l tryptone, $5 \mathrm{~g} / \mathrm{l}$ yeast extract, $8.5 \mathrm{mM}$ $\mathrm{NaCl}, 2.5 \mathrm{mM} \mathrm{KCl}, 100 \mathrm{mM} \mathrm{MgCl} 2,20 \mathrm{mM}$ glucose, $\mathrm{pH}$ 7) was added and cells were incubated for $1 \mathrm{~h}$ at $37^{\circ} \mathrm{C}, 200 \mathrm{rmp}$ before being plated on LB+amp agar plates $(10 \mathrm{~g} / \mathrm{L}$ tryptone, $5 \mathrm{~g} / \mathrm{L}$ yeast extract, $5 \mathrm{~g} / \mathrm{l}$ $\mathrm{NaCl}, 50 \mathrm{\mu g} / \mathrm{l}$ ampicillin, $20 \mathrm{~g} / \mathrm{L}$ agar, $\mathrm{pH}$ 7) and incubated at $37^{\circ} \mathrm{C}$ over night. 3-4 colonies were picked the next day to grow in $4 \mathrm{ml} \mathrm{LB}+a m p$ medium (10 g/L tryptone, $5 \mathrm{~g} / \mathrm{L}$ yeast extract, $5 \mathrm{~g} / \mathrm{L} \mathrm{NaCl}, 50 \mathrm{\mu g} / \mathrm{L}$ ampicillin, pH 7) over night at $37^{\circ} \mathrm{C}, 200 \mathrm{rpm}$. Plasmid DNA was finally purified the next day using the ZR Plasmid Miniprep ${ }^{\text {TM}}$-Classic-Kit (Zymo Research). For validation of a correct coding region, all constructs were sequenced by Microsynth Seqlab, Göttingen.

\subsubsection{In vitro expression}

Channel proteins were expressed in vitro with MembraneMax ${ }^{\mathrm{TM}}$ HN Protein Expression Kit (Thermo Fisher Scientific, Waltham, MA, USA) or Expressway ${ }^{\mathrm{TM}}$ Mini Cell-Free Expression System (Thermo Fisher Scientific, Waltham, MA, USA) according to the manufacturer's instructions, with the difference of halving the total volume of the reaction mixture. To enable proper folding of membrane proteins and to avoid protein aggregation, the expression was performed in the presence of nanodiscs. These were either already included in the kit (MembraneMaxTM HN Protein Expression Kit), containing DMPC lipids, or commercially acquired MSPIDI nanodiscs by Cube Biotech (Cube Biotech GmbH, Monheim, Germany), adjusted to a concentration of $30 \mu \mathrm{mol}$ for the reaction mixture. The latter have a scaffold diameter of 9-10 nm and are available with different assembled lipids (DMPC, DMPG or POPC). A big advantage of nanodiscs is the possibility to isolate membrane proteins without contaminations from the bacterial lysate with the help of a his-tag attached to the membrane scaffold protein (MSP) of the nanodisc. This his-tag allows purification of the nanodisc/channel complex via metal chelate affinity chromatography, using a $0.2 \mathrm{ml}$ HisPur ${ }^{\mathrm{TM}} \mathrm{Ni}$ NTA spin column (Thermo Fisher Scientific, Waltham, MA, USA). This method allows not only a contamination-free expression, but is also much faster than expression systems used in the majority of bilayer experiments, like expression in heterologous systems (Tapper \& George, 2003) or direct isolation from cells (Nelson et al., 1980; Hirano-Iwata et al., 2016). 
Differing to the manufacturer's instructions, wash and elution buffer only contained $25 \mathrm{mM}$ and $250 \mathrm{mM}$ imidazole, dissolved in $\mathrm{dd}_{2} \mathrm{O}$. Keeping the protein-containing nanodiscs in solutions containing salts has been shown to reduce channel activity dramatically (Winterstein et al., 2018). For single channel measurements the first elution fraction was diluted in $250 \mathrm{mM}$ pure imidazole 1:102 $-1: 10^{4}$, depending on protein activity.

\subsection{Planar Lipid Bilayer Method}

In-vitro synthesized and purified channels were analyzed on the single channel level by using artificial, vertical, planar lipid bilayers (PLB) (Montal \& Mueller, 1972). This cell-free, strongly simplified environment allows a quick and simple way of changing various parameters, such as temperature, ion-, lipid- or proton concentration (Hanke \& Schlue, 1993). Fig. 7 schematically shows the bilayer setup. For current measurements, silver/silver chloride (Ag/AgCl) electrodes were used. The electrode of the transcompartment is connected to ground, while the electrode of the cis compartment is directly connected to the preamplifier of a EPC7 plus patch clamp amplifier (HEKA Elektronik GmbH, Lambrecht/Pfalz, Germany). The latter was linked to a personal computer via an InstruTECH LIH 8+8 data acquisition system (HEKA Elektronik GmbH, Lambrecht/Pfalz, Germany) for Analog/Digital (A/D) conversion. Single channel currents were amplified with $100 \mathrm{mV} / \mathrm{pA}$, filtered with $1 \mathrm{kHz}$ and sampled at $5 \mathrm{kHz}$. A detailed description of chamber preparation is described in Winterstein et al. (2018). Bilayers were created using the monolayer folding-technique by Montal \& Mueller (1972) or the pseudo painting/air bubble technique described by Braun et al. (2014) with the help of a bent $25 \mu$ l Hamilton Syringe (Hamilton Bonaduz AG, Bonaduz, Switzerland). Artificial membranes were either exclusively made of 1,2-diphytanoyl-sn-glycero3-phosphocholine (DPhPC, Avanti Polar Lipids, Inc., Alabaster, AL, USA), an uncharged, synthetic lipid with two methylated C 16-fatty acid chains, lacking phase transition from $-120^{\circ} \mathrm{C}$ to $+120^{\circ} \mathrm{C}$, or as a composition of DPhPC and 1,2-diphytanoyl-sn-glycero-3-phospho-L-serine (DPhPS; Avanti Polar Lipids, Inc., Alabaster, AL, USA). DPhPS is also a synthetic lipid like DPhPC, with two methylated C16-fatty acid chains but with a negatively charged head group due to a serine rest. The lipid was always added in a concentration of $15 \mathrm{mg} / \mathrm{ml}$ (solved in n-pentane). All experiments were performed at room temperature $\left(20-25^{\circ} \mathrm{C}\right)$. Bilayer monitoring and application of voltage protocols was carried out with PatchMaster (v2x65, HEKA Elektronik $\mathrm{GmbH}$, Lambrecht/Pfalz). The chambers are completely isolated from each other once a bilayer had been successfully obtained. Its stability was then tested by running a test pulse, which is permanently switching from $-120 \mathrm{mV}$ to $+120 \mathrm{mV}$ for 5 seconds, respectively. These test recordings were made in the absence of protein to rule out the possibility of contaminations or other artifacts like lipid pores, which would influence the measurement (Heimburg, 2010). Only bilayers without disturbances or current leakages were considered for measurements. Should this be the case, a small amount (2-3 $\mu$ l) of the protein dilution was added to the trans-chamber with a bent Hamilton syringe. After reconstitution of a single channel into the bilayer, channel-specific voltage protocols were applied (Table 1). Due to the fact that channel insertion nearly always occurs in the same direction (Winterstein, 2019), the trans-chamber represents the extracellular side, the cis chamber correspondingly the intracellular side of the cell. 
Table 1 - Overview of channel-specific voltage protocols applied with PatchMaster for single channel measurements.

\begin{tabular}{|c|c|c|c|}
\hline Channel name & Voltage range & Voltage increment & Duration per voltage \\
\hline $\mathrm{KCV}_{\mathrm{PBCV}-1}+$ mutants & $-160 \mathrm{mV}$ to $+160 \mathrm{mV}$ & $20 \mathrm{mV}$ & $5 \mathrm{~min}$ \\
\hline KCVGNLD + mutants & $-160 \mathrm{mV}$ to $+260 \mathrm{mV}$ & $20 \mathrm{mV}$ & $\begin{array}{c}10 \mathrm{~s} \\
1 \mathrm{~min}\end{array}$ \\
\hline $\mathrm{KCV}_{\text {MT} 325}+$ mutants & $-160 \mathrm{mV}$ to $+260 \mathrm{mV}$ & $20 \mathrm{mV}$ & $\begin{array}{l}10 \mathrm{~s} \\
1 \mathrm{~min}\end{array}$ \\
\hline KCVNTS & $-160 \mathrm{mV}$ to $+260 \mathrm{mV}$ & $20 \mathrm{mV}$ & $10 \mathrm{~s}$ \\
\hline KcsA E71A & $-160 \mathrm{mV}$ to $+260 \mathrm{mV}$ & $20 \mathrm{mV}$ & $10 \mathrm{~s}$ \\
\hline
\end{tabular}

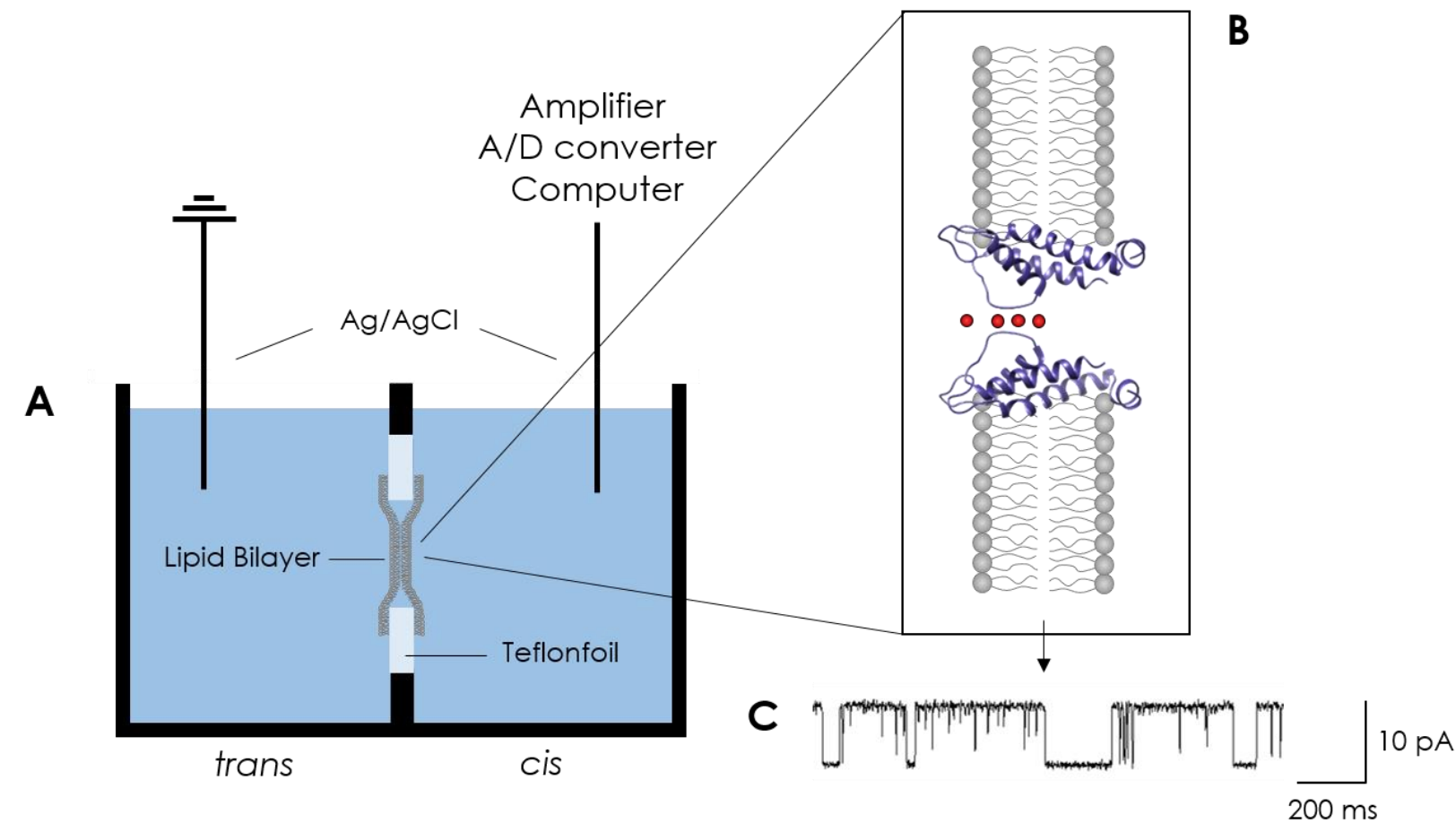

Figure 7 - Schematic composition of a bilayer setup. (A) Two Teflon chambers are separated from each other by a Teflon foil, in which a $100 \mu \mathrm{m}$ sized hole has been burned previously. The electrode of the trans chamber is connected to ground, while the electrode of the cis chamber is used to apply voltage. Formation of a lipid bilayer over the hole isolates the chambers completely. Successful reconstitution of a single channel into the bilayer (B) allows ion flow (red spheres) across the membrane after voltage application, resulting in stochastic gating events (C).

\subsubsection{Analyses}

Single channel traces were analyzed with the custom-made program KielPatch (version 3.2 (ZBM/2011), center for biochemistry and molecular biology, CAU Kiel, Germany). Further analyses and data editing 
were performed with Matlab (MathWorks, Natick, MA, USA), Microsoft Excel and Microsoft PowerPoint (Microsoft Corporation, Redmond, WA, USA). Images of single channel traces were generated with PatchMaster.

The slope of the corresponding i/V curves in the linear range represents the single channel conductance $(\gamma)$ of a channel; it can be calculated with

$\gamma=\frac{\Delta \mathrm{l}}{\Delta \mathrm{V}}$

For calculation of the open probability $\left(\mathrm{P}_{\circ}\right)$ the mathematical formula

$P_{\circ}=\frac{t_{\text {open }}}{t_{\text {total }}}$

where topen is the summary of times the channel spends in its open state and total the duration of the measurement for each voltage, is used. KielPatch detects the transitions of a single channel between its open and closed state at a given voltage. With this information the software calculates the total amount of time spent in each state, respectively.

For fitting the data, the weighted squared error sum $\chi^{2}$ was calculated with

$\chi^{2}=\stackrel{(y-\bar{y})^{2}}{\Delta y}$

with $y$ being the simulated data, $\bar{y}$ the arithmetic mean value of the measured data and $\Delta y$ the corresponding arithmetic standard deviation of the mean value. To achieve the most suitable fit, $\chi^{2}$ was minimized. 


\section{Results}

\subsection{In vitro protein expression into nanodiscs}

Ion channels are involved in many essential processes in biological systems, e.g. maintenance of the membrane potential or electrical signaling (Hille, 2001). Since many diseases concerning the cardiac and nervous systems are caused by mutations within ion channels (Ashcroft, 1999), it is crucial to understand the structure/function relationships of these proteins. Today it is possible to measure currents of single ion channels across biological or artificial membranes. The history of electrophysiology goes back to Hodgkin \& Huxley (1939), who were one of the first to measure an action potential on a giant squid axon. Inspired by the research of Hodgkin \& Huxley, Neher \& Sakmann were able to establish the patch clamp method (1976). This method allows measurements of currents across the entire cell membrane as well as of individual channels, created by a protein of interest (POI) that the cells were previously transfected with. While this method is today well-established and one of the most prominent measuring tools concerning the investigation of ion channel activity, a variety of electrophysiological methods have developed in parallel, including the prevalent PLB method. The basic principle was established by Müller et al. (1962). This method has the advantage that it provides a highly reduced system in which a variety of parameters can be controlled and easily modified, e.g. lipid composition, buffer solution, $\mathrm{pH}$ or temperature. Due to the simple construction of the setup, manipulation of external and internal solution is equally fast and requires no great effort (Williams, 1994). It is therefore a useful method to study ion channels on a single channel level in different lipid and buffer compositions. In addition, the effect of drugs can be monitored on a single channel level (Zakharian, 2013). While the basic principle has hardly changed from its first report until today, there have been many attempts to improve and simplify the PLB method (Hirano-Iwata et al., 2015). These include improvement of the mechanical stability (Tien et al., 1991; Schuster et al., 1999; Khan et al., 2016), a reduction of the size of the measuring device (Mach et al., 2008) or improvement of the temporal resolution of channel recordings (Hartel et al., 2018).

Regardless of the measuring setup, the $\mathrm{POI}$ needs to be provided in some way. It can be either directly isolated from cells into vesicles (Nelson et al., 1980; Hirano-Iwata et al., 2016) or expressed in different heterologous systems (Kingsman et al., 1987; Needham et al., 1992; Pfeifer, 1998; Shatzman, 1990). Commonly used expression systems are e. g. Escherichia coli (Gordon et al., 2008; Chen, 2012) Lactococcus lactis (De Vos, 1999; Kunji et al., 2003) or Pichia pastoris (Cregg et al., 2000; Pagliuca et al., 2007; De Schutter et al., 2009). The entire process from protein expression and subsequent purification to the actual single channel measurement after reconstitution into a PLB can be very time consuming. Lately, cell free translation systems offer an alternative strategy for expression of proteins (Klammt et al., 2004; Katzen et al., 2005; Sobhanifar et al., 2010). Protein expression takes place in vitro and can subsequently be used for successful reconstitution into PLBs after purification (Cheley et al., 1999; Focke et al., 2016). A disadvantage of these approaches is the degree of contamination. Proteins from the lysate in which the POI was synthesized have been found in the purified samples (Andersen et al., 1986; Mateo et al., 2001; Accardi et al., 2004; Bolanos-Garcia \& Davies, 2006). An additional challenge with membrane proteins is to keep them stable and active in a suitable environment; held in detergent they are known 
to denature (Bill et al., 2011) and expression in e. g. E. coli, without their natural lipid environment, leads to aggregation of the protein in the cytoplasm (Seddon et al., 2004).

In Winterstein et al. (2018) we have used a novel technique which speeds up the process of protein expression. This was achieved by combining E. coli extract for cell free, in vitro protein expression with commercially available nanodiscs. Nanodiscs are lipid bilayer patches (here: DMPC, DMPG, POPC) of different sizes (here: 9-10 nm) that are held together by a membrane scaffold protein (MSP) (Hagn et al., 2013). By adding the nanodiscs to the expression system lysate, they provide a stable, detergent-free, native environment for correct folding of the membrane proteins and thus avoid protein aggregation. Another advantage of the nanodiscs is an additional his-tag which is attached to the MSP. It allows purification of the nanondisc/protein complex via metal chelate affinity chromatography leaving the POI in its native condition. The process from protein expression to finished purification is done in only a few hours, so measurements can start on the same day. This technique has already been successfully applied for single channel characterization of the bacterial channel KcsA (Dörr et al., 2014). In the study of Winterstein et al. (2018), we show that this rapid, contamination-free method provides an abundant amount of functional protein in nanodiscs for electrophysiological investigations in artificial, PLBs.

\subsubsection{Target membrane properties determine channel function}

As mentioned before, in vitro expression of membrane proteins took place in the presence of nanodiscs. This enables proper protein folding and prevents protein aggregation. In Winterstein et al. (2018) we show that not the lipid properties of the nanodisc determines channel function, but the target membrane. Three different types of nanodiscs were purchased from Cube Biotech, possessing the same size $(9-10 \mathrm{~nm})$ and membrane scaffold protein, but differing in lipid composition (DMPC, DMPG and POPC). The MembraneMax ${ }^{\mathrm{TM}} \mathrm{HN}$ Protein Expression Kit already contains nanodiscs of unknown size with DMPC lipids; they are further referred to as MM (MembraneMax). To see whether the lipid environment of the nanodics or the target membrane have an influence on channel gating, the small viral $\mathrm{K}^{+}$channel KcVNTs (Braun et al., 2014) and the bacterial $\mathrm{K}^{+}$channel mutant KcsA E71 A, which lacks inactivation (Cordero-Morales et al., 2006), were concomitantly expressed in four different approaches, each containing a different type of nanodisc. The purified proteins in their corresponding nanodisc were then examined on the single channel level with the PLB method in either pure DPhPC or 3:1 DPhPC:DPhPS mixed membranes, comparing conductance and open probability for each approach.

$\mathrm{KCVNTS}_{\mathrm{N}}$ is a viral $\mathrm{K}^{+}$channel encoded by a chlorella virus isolated from a potassium rich lake in Nebraska. It consists of only 82 amino acids per subunit but still forms a functional homotetramer with the typical characteristics of $\mathrm{K}^{+}$channels (Greiner, 2011). Previous studies have shown that the channel is fully embedded in the PLB (Braun et al., 2014) and is thus expected to be sensitive towards changes in the membrane. The channel was expressed in the presence of four different nanodiscs (MM, DMPC, DMPG, POPC) and measured with the PLB method in pure DPhPC membranes. Representative single channel traces of KcVNTs (fig. 8 A) show that the channel exhibits the same sort of spontaneous fluctuations in DPhPC membranes independent of the nanodisc it was expressed in. Corresponding i/V relation and open probability $\left(\mathrm{P}_{\circ}\right)$ are shown in fig. $8 \mathrm{C} \& \mathrm{D}$, confirming the visual impressions of the single channel 
traces. Comparison of the four different protein expressions show no significant differences. The unitary single channel conductance is in the same range for all measurements (fig. 8 B, unicolored bars), as is the mean open probability (fig. 8 B, striped bars).

A

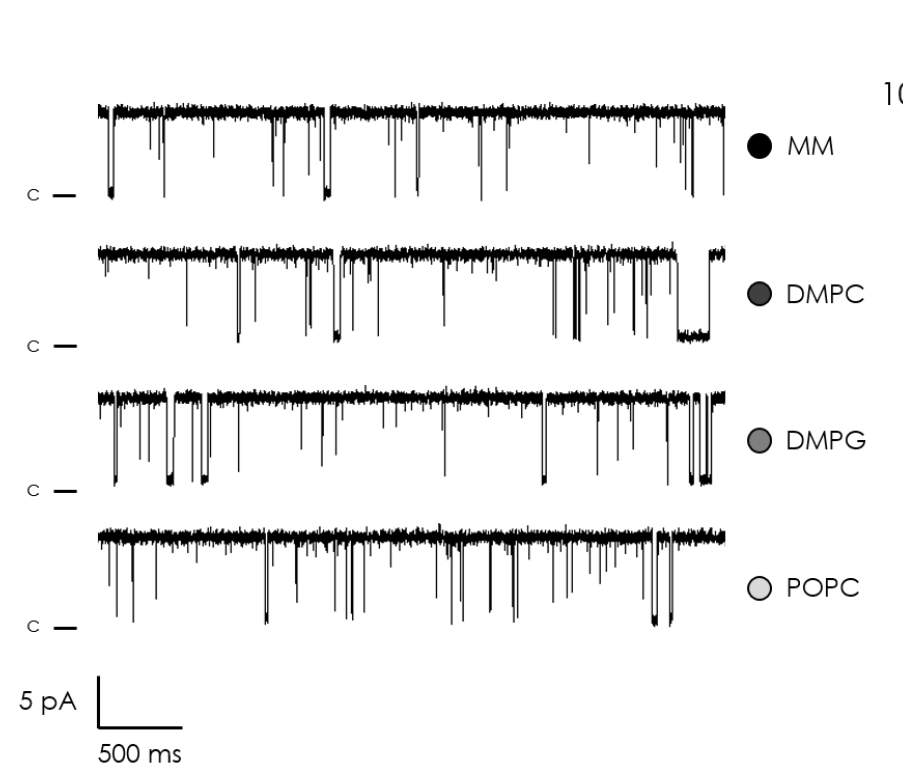

B

$$
\mathrm{G} / \mathrm{pS} \quad \mathrm{P}_{\mathrm{O}}
$$

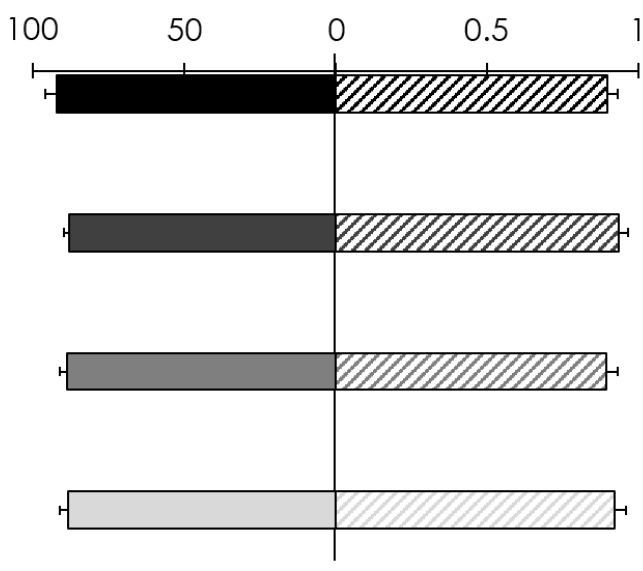

C

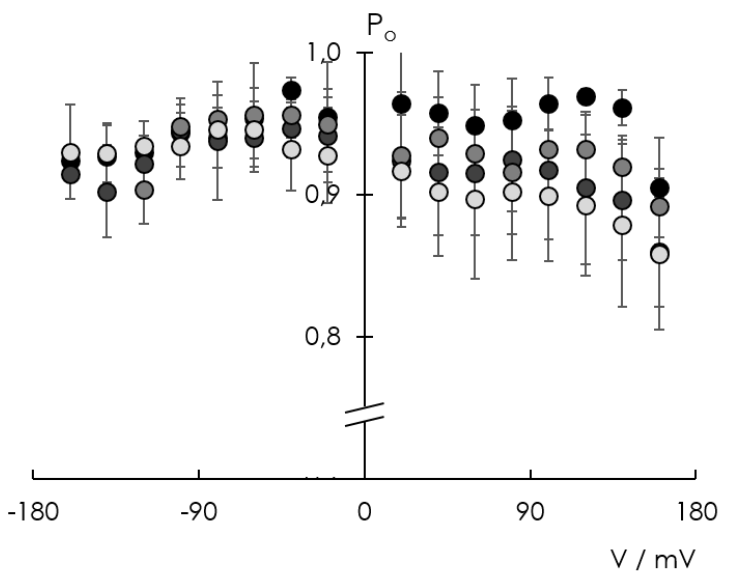

D

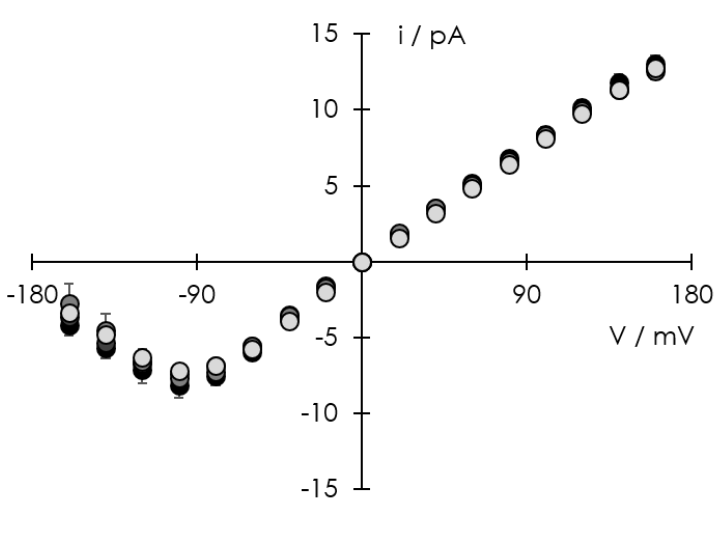

Figure 8 - Reconstitution of the viral $\mathrm{K}^{+}$channel KCVNTs from different nanodiscs in pure DPhPC membranes. (A) Exemplary current traces of KcVNTs at $+100 \mathrm{mV}$. c represents the open state. The protein was expressed in four different approaches, each containing a different nanodisc (MM, DMPC, DMPG, POPC). For better visualization, data was subsequently filtered with $500 \mathrm{~Hz}$. (B) Corresponding unitary single channel conductance (unicolored bars) and open probability (striped bars). Corresponding open probability (C) and i/ $V$ relation (D). Data are mean values \pm standard deviation for $n$ independent measurements (MM: $n=3$; DMPC: $n=4$; DMPG: $n=4$; POPC: $n=4$ ). Measurements were performed under symmetrical conditions with $100 \mathrm{mM} \mathrm{KCl}$ and $10 \mathrm{mM} \mathrm{HEPES}$ at $\mathrm{pH} 7$. 
This experiment has shown that the small viral $\mathrm{K}^{+}$channel KcVNTs could successfully be expressed into nanodiscs and incorporated into pure DPhPC membranes for single channel measurements. Despite the different nanodiscs, no difference in unitary single channel conductance or open probability could be seen. To examine if this method of expression is also applicable for other, bigger channels, the experiment was repeated with a mutant of the bacterial KcsA channel, KcsA E71 A. KcsA is a small bacterial $\mathrm{K}^{+}$ channel. Since the first crystal structure of a $\mathrm{K}^{+}$channel (Doyle et al., 1998) was obtained from KcsA, it became a model channel for research on structure/function correlates in this family of channels. The basic structure of KcVNTs and KcsA is the same: both channels only consist of two transmembrane helices, representing the pore module of more complex $\mathrm{K}^{+}$channels. However, KcsA possesses a C-terminal cytoplasmic domain consisting of $\sim 40$ amino acids. $\mathrm{Kcs} A$ is a proton activated $\mathrm{K}^{+}$channel with a very low open probability. Mutation from glutamate at position 71 to alanine strongly increases the open probability, due to the abolishment of channel inactivation (Cordero-Morales et al., 2006). The previously described experiment for $\mathrm{K}_{\mathrm{C} V \mathrm{NTS}}$ was repeated with the noninactivating mutant KcsA E7lA. The channel was expressed in the presence of four different nanodiscs (MM, DMPC, DMPG, POPC) and measured with the PLB method in pure DPhPC membranes. It has been reported that anionic lipids are necessary for KcsA channel function (Valiyaveetil et al., 2002), so an additional experiment was performed where KcsA from one of the expression approaches (DMPC nanodiscs) was added to a membrane made of a 3:1 mix of DPhPC and DPhPS. DPhPS is also a synthetic lipid like DPhPC, with two methylated C16-fatty acid chains but with a negatively charged head group due to a serine rest. Fig. 9 A shows representative single channel traces of both experiments. While the unitary single channel conductance for the measurements in pure DPhPC membranes (MM, DMPC, DMPG, POPC) show the same channel fluctuations, the unitary single channel conductance of KCSA in 3:1 DPhPC:DPhPS membranes (DPhPS) is higher. This can be confirmed by comparison of the i/V relation (fig. 9 B) and conductance (fig. 9 C). Interestingly, there is no difference seen in the open probability of KCSA E7 I A, for uncharged and charged membranes (data not shown). 
A

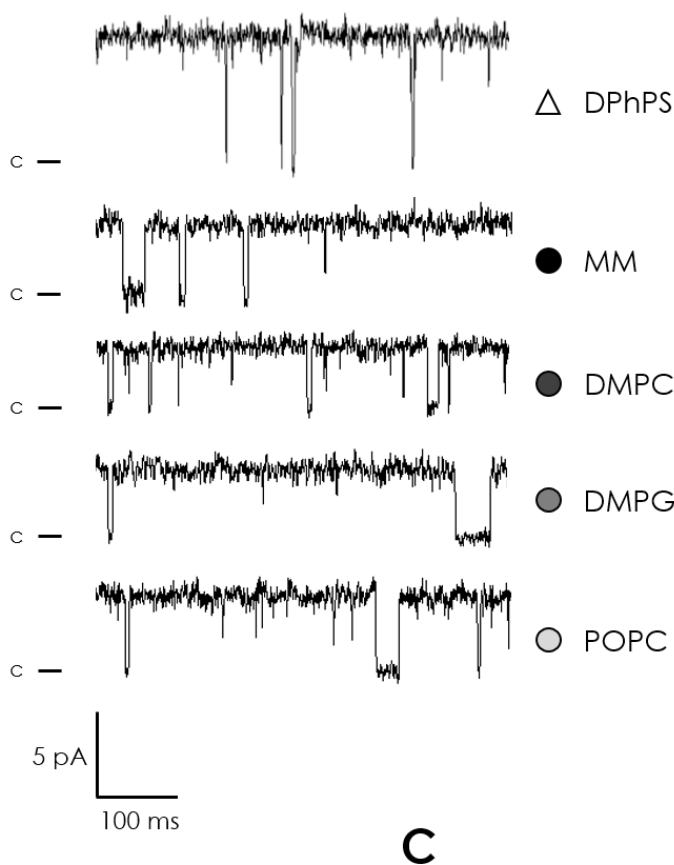

B

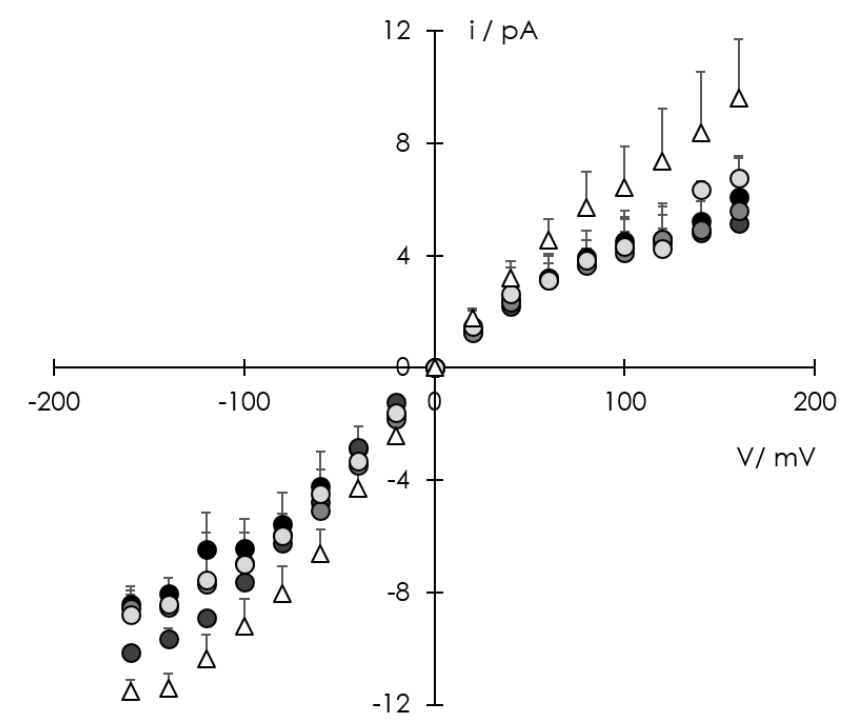

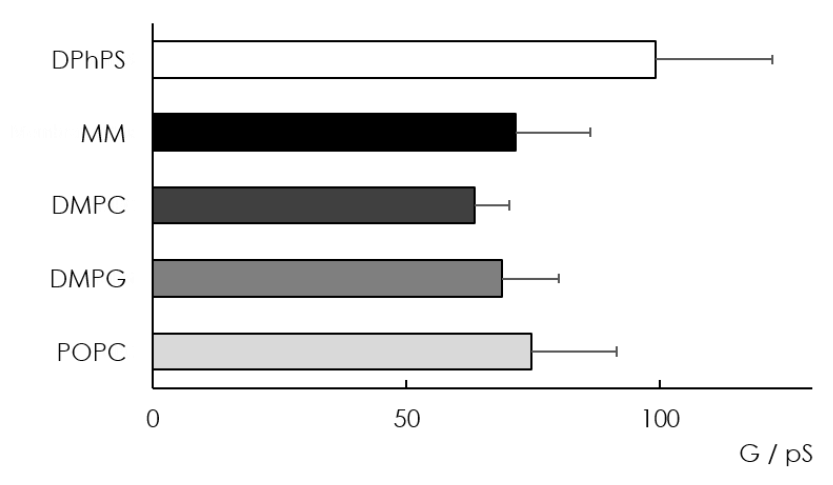

Figure 9 - Reconstitution of the bacterial $\mathrm{K}^{+}$channel KcsA E71A in different PLBs. (A) Exemplary current traces of KCSA E7I A at $+100 \mathrm{mV}$. c represents the closed state. The protein was expressed in four different approaches, each containing a different type of nanodisc (MM, DMPC, DMPG, POPC; circles). In the top trace, the target membrane was made of a 3:1 mix of DPhPC and the negatively charged DPhPS (triangle). Protein was added from nanodiscs that consisted of DMPC. For the other four current traces the target membrane was made of pure DPhPC. Protein was added from all four expression approaches. For better visualization, data was subsequently filtered with $500 \mathrm{~Hz}$. Corresponding i/ $\mathrm{V}$ relation (B) and unitary single channel conductance (C). Data are mean values \pm standard deviation for $n$ independent measurements (DPhPS: $n=3 ; M M: n=6$; DMPC: $n=4$; DMPG: $\mathrm{n}=4$; POPC: $\mathrm{n=3}$ ). Measurements were performed under symmetrical conditions with $90 \mathrm{mM} \mathrm{KCl}$ and $10 \mathrm{mM} \mathrm{CH}_{3} \mathrm{COOK}$ at $\mathrm{pH} 4$.

The next experiment addresses the question of whether the reconstitution into different nanodiscs affects channel insertion into the target membrane. To test this, a higher concentration ( $2 \mu$ l of the first elution) of protein from the four KcVNTs elutions of the protein purification step was added to pure DPhPC membranes. For single channel measurements the first elution fraction is normally diluted 1:104, due to the high amount of protein after expression. For the experiments reported in fig. 10, the membrane was held 
at $+60 \mathrm{mV}$ and the duration of ten channels to insert (corresponding to $\sim 50 \mathrm{pA}$ ) was monitored. Fig. $10 \mathrm{~A}$ shows an example of the time dependent channel insertion. Protein addition is marked by the continuous red bar, which explains the large noise. A few seconds after protein addition the first channel insertion can be observed (blue arrow), immediately followed by stepwise reconstitution of further channels. The red dotted line marks a current of $50 \mathrm{pA}$ and the red arrow marks insertion of the tenth channel. The displayed graph in fig. 10 A shows the insertion efficiency of KcVNTs expressed in POPC, DMPG and DMPC nanodiscs. While the 50 pA mark is reached after just a few seconds for channels expressed in DMPG and DMPC nanodiscs, the insertion efficiency for channels in POPC nanodiscs is significantly lower, implying that protein expression in the presence of POPC nanodiscs is less suitable for subsequent measurements. DMPC and DMPG both possess two fully saturated C-14 fatty acid chains. The thickness of the membrane is therefore the same. They differ only in their head groups, in DMPG being negatively charged while DMPC is neutral. POPC consists of two fatty acid chains of a different length, a saturated C-16 chain and a monounsaturated C-18 chain. This means POPC bilayers are slightly thicker than DMPC and DMPG. The membrane thickness might influence channel transition from the nanodisc into the target membrane.

To gain more information about the storage of proteins in nanodiscs after expression, the elution batches were split into half. One aliquot was stored at $-20^{\circ} \mathrm{C}$, while the other one was kept in the fridge $\left(+4^{\circ} \mathrm{C}\right)$. Four days later, measurements were performed with both preparations. For these measurements, a higher concentrated $(1: 100)$ dilution was added to a pure DPhPC bilayer held at $+120 \mathrm{mV}$. Fig. $10 \mathrm{~B}$ shows a representative result. Multiple additions of the 1:100 dilution which was stored at $-20^{\circ} \mathrm{C}$ failed to show any channel activity (left, open triangle). Only a few seconds after the first addition of the 1:100 dilution kept at $+4{ }^{\circ} \mathrm{C}$ insertion of multiple channels (right, black triangle) could be recorded. This proves that after protein expression the elutions should not be frozen but stored at $+4{ }^{\circ} \mathrm{C}$ to obtain channel activity. 
A

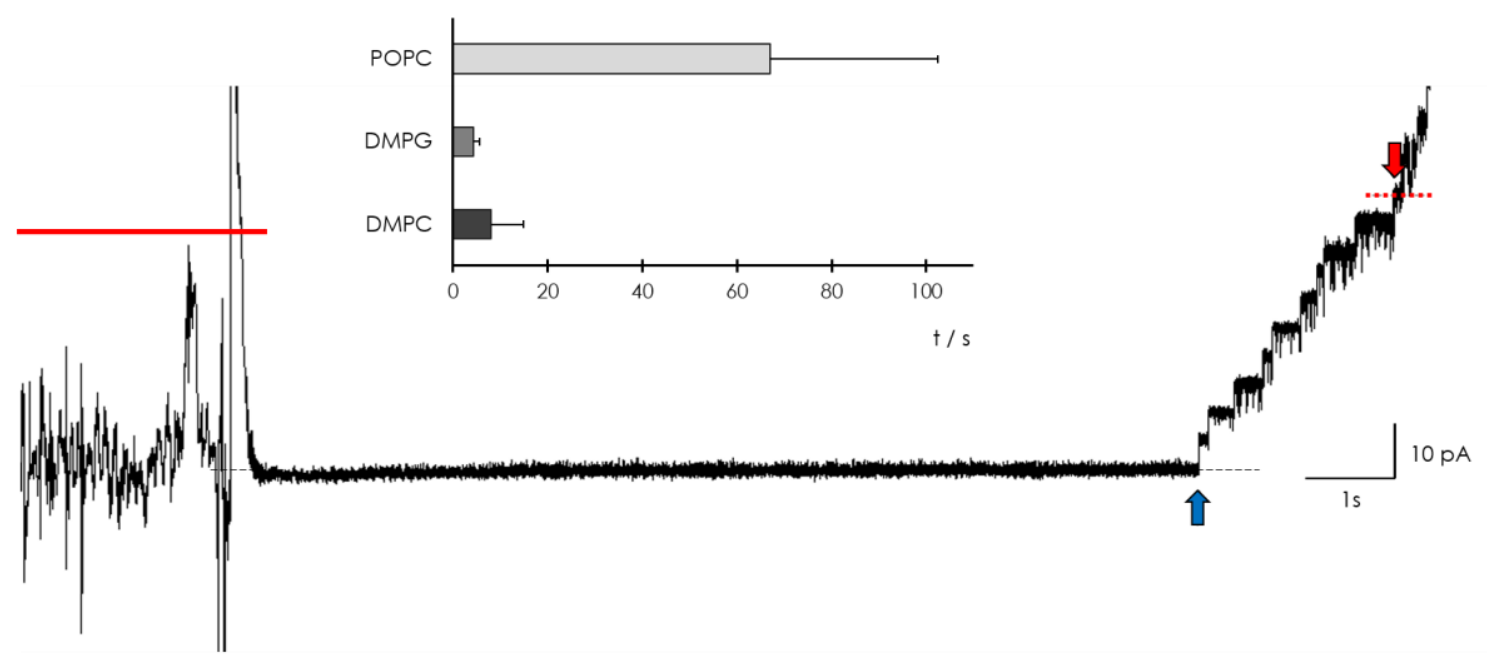

B

$\boldsymbol{\nabla}$

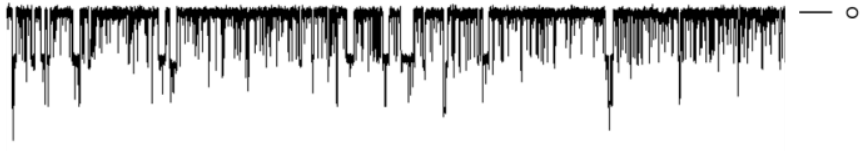

$\nabla$

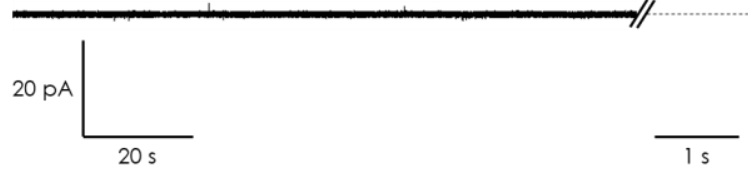

Figure 10 - Insertion efficiency and storage of $\mathrm{KCV}_{\mathrm{NTS}}$ in different nanodiscs. (A) Exemplary recording of KCVNTS reconstitution from DMPC nanodiscs into a pure DPhPC bilayer kept at $+60 \mathrm{mV}$. Channel addition is marked with a red bar, the black dotted line marks $0 \mathrm{mV}$. After a few seconds the first channel incorporates (blue arrow). The red arrow indicates incorporation of the 10th channel, which is equivalent to $\sim 50 \mathrm{pA}$ (red dotted line). The efficiency of channel reconstitution of KcVNTs expressed in different nanodiscs (POPC, DMPC, DMPG) is shown in the inserted graph. Data are mean values \pm standard deviation of $n$ independent measurements (POPC: $n=5 ; D M P C: n=5$; DMPG: $n=5)$. (B) Exemplary traces of reconstitution of KCVNTS expressed in MM nanodiscs after storage at $-20{ }^{\circ} \mathrm{C}$ (left, open triangle) and $+4{ }^{\circ} \mathrm{C}$ (right, black triangle). Recordings started a few seconds after channel insertion and DPhPC membranes were held at $+120 \mathrm{mV}$. The dotted line indicates $0 \mathrm{mV}$. Measurements were made under symmetrical conditions with $100 \mathrm{mM} \mathrm{KCl}$ and 10 $\mathrm{mM}$ HEPES at $\mathrm{pH}$ 7. For better visualization, data was subsequently filtered with $500 \mathrm{~Hz}$.

\subsubsection{Expression and successful reconstitution of a blue-light-induced $\mathrm{K}^{+}$channel (BLINK1)} in PLB

The experiments mentioned above have shown that small viral $\mathrm{K}^{+}$channels, as well as the bacterial $\mathrm{K}^{+}$ channel KcsA can successfully be expressed in vitro in the presence of different nanodiscs. From there they can be reconstituted into different lipid bilayers for functional studies. Hereby the properties of the target membrane determine channel function, not the nanodiscs in which the channels are expressed. 
An additional candidate that was examined with this expression method is the blue-light-induced $\mathrm{K}^{+}$ channel 1, short BLINK 1. It was designed by Cosentino et al. (2014) and is the predecessor of the improved channel BLINK2 (Alberio et al., 2018).

$\mathrm{K}^{+}$channels are known to respond to a variety of physiological stimuli, which modulate the characteristic gating properties of the channels. For this purpose, channels are equipped with specific sensor domains which are attached to the pore module. While the pore region of $\mathrm{K}^{+}$channels is highly conserved throughout evolution (Heginbotham et al., 1994), sensor domains are responsible for the great variety of channels (Ohndorf \& MacKinnon, 2005). Several successful approaches have been made to attach sensor domains to different channel pores, in order to modify their gating properties (Arrigoni et al., 2013; Ohndorf \& Mackinnon, 2005). An attractive external stimulus is light. Successful fusion of a light sensing domain to a functional $\mathrm{K}^{+}$channel would allow regulation of several cellular activities with high spatial and temporal resolution. With this background BLINK 1 was designed. It is composed of the small viral $\mathrm{K}^{+}$ channel KCVPBCV-1, which forms the simple pore module. The LOV2-Ja photosensory domain and an additional myristoylation/palmitoylation sequence for membrane coupling are attached to the slide helix at the N-terminus of $\mathrm{KCV}_{\mathrm{PBCV}-1}$ by a short linker. The channel can be activated upon exposure of blue light, in a reversible manner (Cosentino et al., 2014). Electrophysiological characterization of BLINK 1 was so far only performed with whole-cell patch clamp measurements in HEK293T cells. Single channel measurements had only been performed with a precursor of the channel (Cosentino et al., 2014). For this reason, it was interesting to examine the single channel properties of BLINK 1 with the PLB method.

BLINK 1 was expressed in vitro in the presence of MM nanodiscs as described above, with additional 100 $\mu \mathrm{M}$ Riboflavin 5'-monophosphate sodium salt. The target membrane consisted of pure DPhPC. Measurements were performed under symmetrical conditions with $100 \mathrm{mM} \mathrm{KCl}$ and $10 \mathrm{mM} \mathrm{HEPES}$ at $\mathrm{pH}$ 7. The results were compared to single channel measurements of KCVPBCV-1, which represents the pore module of BLINK1.

Multiple measurements of BLINKI showed two distinct gating modes. Representative single channel traces of both gating types are shown in fig. 11 A with two different temporal resolutions. At negative membrane potentials, the gating types are very similar. Gating is characterized by very long closed events, in the range of seconds, with bursts of activity. During these bursts, the dwell times of closed events are strongly reduced and open events occur with dwell times in the ms range. For positive voltages, the gating types differ strongly. Type 1 has a much higher open probability. Long closed times still occur, interrupted by longer bursts that consist of a higher number of open events with shorter dwell times than at negative voltages, but longer dwell times than seen for gating type 2 . The open probability for type 2 is slightly reduced at positive voltages. Closed events are not as long as for type 1, mainly in the long ms range, but the number of open events is less and the dwell times of these are very short, partially too short to be fully resolved. Both gating types have a voltage dependency. For type 1 it is more pronounced: the open probability increases from $\sim 5 \%$ at negative voltages up to $\sim 20 \%$ at positive membrane potentials. Type 2 only has a slight voltage dependency, inverse to that of type 1. Here the open probability is reduced from $\sim 5 \%$ at negative voltages to $\leq 1 \%$ at positive voltages. The unitary single channel 
conductance of both gating types is the same. These observations can be confirmed by the corresponding i/V relation (fig. 11 B, black circles) and open probability curves (fig. 11 C, grey circles for different gating type corresponding to fig. $11 \mathrm{~A}$ ). Since the pore module of BLINK 1 is composed of the viral $\mathrm{K}^{+}$channel KCVPBCV-1, data from the single channel recordings of BLINK 1 was compared to the wild type channel (fig. 11 B\&C, open triangles). The unitary single channel conductance of BLINK 1 is with 186.9 \pm 16.7 pS insignificantly higher than the wild type channel with $174.4 \pm 9.1$ pS. Interestingly, the open probability of gating type 2 is exactly like the wild type channel. Linking of the light sensing domain to the $\mathrm{N}$-terminus of KCVPBCV-1 seems to induce a second gating type with a higher open probability, which is inversed to that of the wild type channel.

A
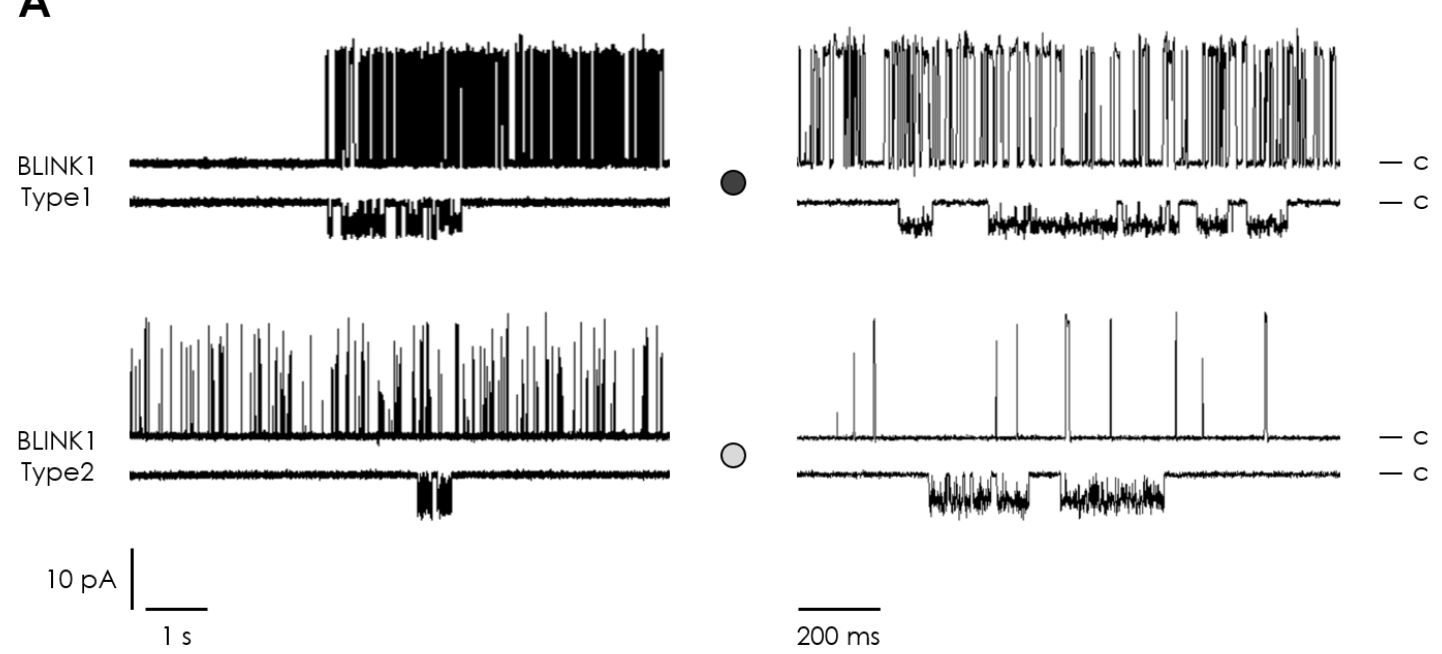

B

C
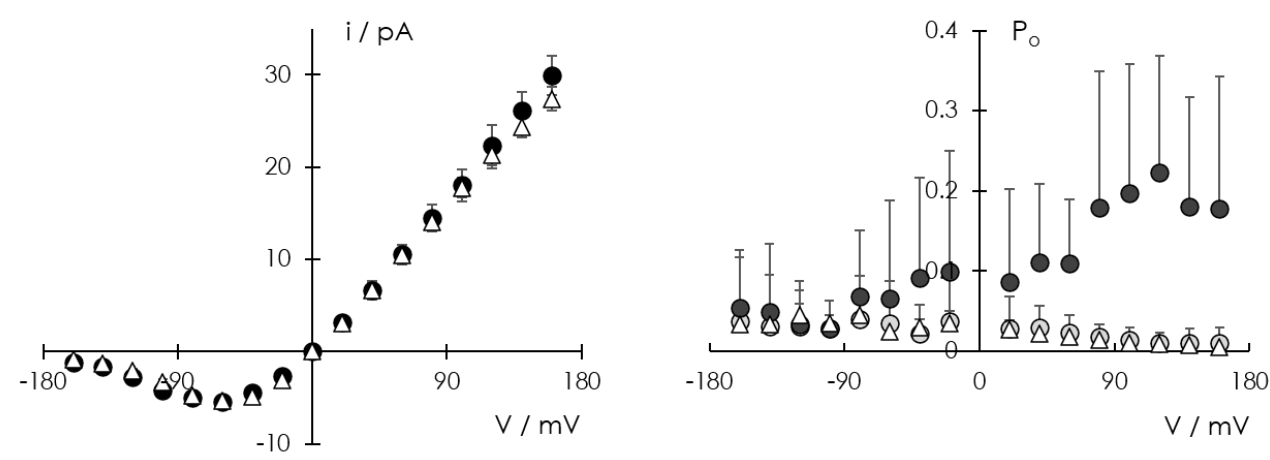

Figure 11 - Single channel properties of the blue-light-induced $\mathrm{K}^{+}$channel 1 (BLINK1) compared to KCVPBCV-1. (A) Exemplary single channel traces of BLINK1 expressed in MM disCs with different temporal resolutions at $\pm 100 \mathrm{mV}$. The channel shows two different gating modes, type 1 (top) is voltage-dependent with a higher open probability at positive voltages and type 2 (bottom) has a lower mean open probability with a slight inverted voltage dependency. c marks the closed state. For better visualization, data was subsequently filtered with $500 \mathrm{~Hz}$. Corresponding i/V relation (B) and open probability (C). Gating type 1 is represented by dark grey circles, gating type 2 by light grey circles and $K_{C V_{P B C V}-1}$ by open triangles. Data are mean values \pm standard deviations for $n$ independent measurements (BLINK 1: $n=13$; type 1:n=6; type 2: $\left.n=7 ; K_{C V P B C V}-1: n=8\right)$ ). Measurements were performed under symmetrical conditions with $100 \mathrm{mM} \mathrm{KCl}$ and $10 \mathrm{mM} \mathrm{HEPES}$ at $\mathrm{pH} 7$. 
Since BLINK 1 was created to be activated by light, measurements were performed in darkness and after illumination with blue light. After successful incorporation of a channel into the membrane, the setup was completely darkened. After waiting for 10 minutes, voltage protocols were applied. After the measurements in darkness, the membrane was illuminated with blue light and measurements were repeated with the same voltage protocols. Unfortunately, no additional channel activation was seen. There was no difference in the measurements before and after blue light illumination for both gating types (fig. 12, Gdark: $185 \pm 15.1$ pS; Glight: $200 \pm 6.3$ pS).

A

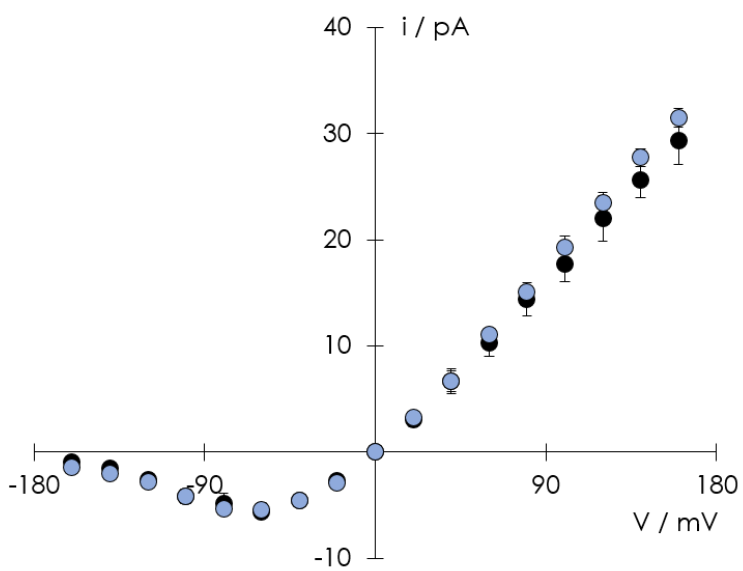

B

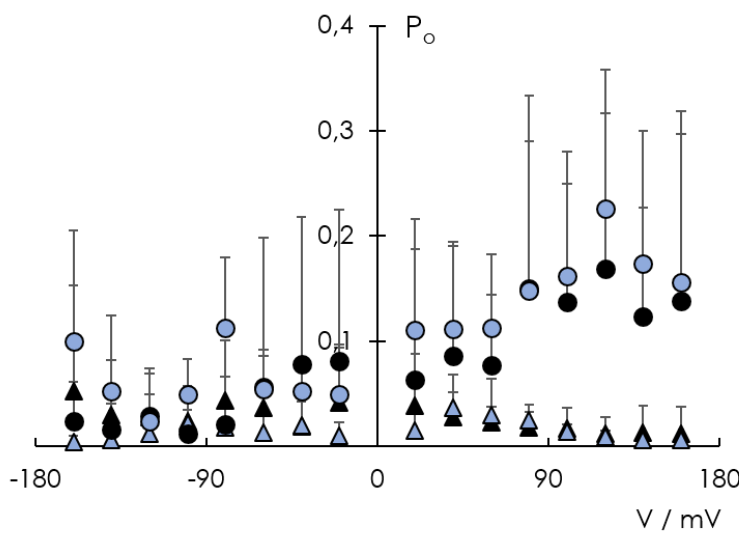

Figure 12 - Single channel properties of BLINK1 after blue light exposure. (A) i/V relation and (B) open probability of BLINK1 before (black circles/triangles) and after (blue circles/triangles) $30 \mathrm{~s}$ of exposure to blue light. While the conductance is the same for both gating types of BLINK 1 (A, circles), the open probability shows two gating types (B type 1: circles; type 2: triangles). Data are mean values \pm standard deviation of $n$ independent measurements (type 1: $n=4$; type $2: n=3$ ). Measurements were performed under symmetrical conditions with $100 \mathrm{mM} \mathrm{KCl}, 10 \mathrm{mM} \mathrm{HEPES}$ at pH 7.

\subsubsection{Discussion}

The combination of in vitro protein expression with addition of nanodiscs turns out to be an efficient, fast and straightforward way to obtain a POI for reconstitution in PLBs. From the start of expression until the end of purification it only takes a few hours, so that protein expression and subsequent measurements can be performed all on the same day. Our data confirm that this method works not only for small viral $\mathrm{K}^{+}$channels (KCVNTS), but also for bigger channels, including the model bacterial $\mathrm{K}^{+}$channel (KcsA E71A) and the synthetic blue light sensitive channel (BLINK1). All channels could be successfully expressed in different nanodiscs (MM, DMPC, DMPG, POPC), showing the same channel fluctuations in PLBs, independent of the nanodisc lipid it was expressed in. The functional parameters of the channels obtained by this procedure are all in agreement with data from the literature. KcVNTs has been expressed and purified in Pichia pastoris and subsequently measured in DPhPC membranes (Braun et al., 2014), with results matching those from our measurements. KCSA E7 I A has previously been expressed and purified in Escherichia coli and reconstituted via liposomes (Cordero-Morales et al., 2006) or nanoscaled 
apolipoprotein-bound bilayers (Banerjee \& Nimigean, 2011) which our results are also in agreement with. Further experiments show that these results are not just obtained with the PLB method, but also work with an alternative approach, namely the contact bubble bilayer (CBB) method (Winterstein et al., 2018). An additional benefit of the present nanodisc method is the lack of contamination. So far, no contaminations could be detected during several years of measurements using this method. This is a great advantage over conventional methods, which often suffer from contaminations from the isolation procedure (Andersen et al., 1986; Mateo et al., 2001; Accardi et al., 2004; Bolanos-Garcia \& Davies, 2006).

For ideal storage, the protocol of protein purification was slightly modified: different from the manufacturer's instructions, wash and elution buffer only contained $25 \mathrm{mM}$ and $250 \mathrm{mM}$ imidazole, respectively, dissolved in $\mathrm{ddH}_{2} \mathrm{O}$, without PBS. Experiments have shown that this combination yields the best results. If PBS is present during the washing process, the amount of protein in the final elution is about 10-fold lower than without PBS (Winterstein et al., 2018). A possible explanation would be that salts remove some of the nanodiscs in the column already during the washing process. Also, while for immediate use after expression storage in salt-containing buffer does not affect channel activity, experiments have shown that it reduces channel activity dramatically and progressively over hours (Winterstein et al., 2018). Properly stored at $4{ }^{\circ} \mathrm{C}$, the proteins can maintain their function for several years.

In all cases, the target membrane determined channel function, not the lipid of the nanodisc. In a new study from Winterstein (2019) it is shown that KcVNTS has an increased unitary single channel conductance when exposed to negatively charged membranes. Measurements with identical buffer concentrations were performed with KcVNis expressed in DMPC nanodiscs and measured in pure DPhPS and DPhPG $(1,2-$ diphytanoyl-sn-glycero-3-phospho-(1'-rac-glycerol)) membranes. Both lipids have negatively charged head groups. When measured in both membranes, the unitary single channel conductance was equally increased compared to DPhPC membranes. This emphasizes the findings in Winterstein et al. (2018) that not the lipid from the nanodisc, but of the target membrane composition is critical for channel function. No such increase could be seen after expression of KcVNis in the presence of nanodiscs containing the negatively charged DMPG lipid. KCSA E7IA also showed an increase in unitary single channel conductance when the target membrane contained anionic lipids (fig. 9). This could not be seen in measurements in pure DPhPC membranes, after expression of KCSA E71A in the presence of DMPG nanodiscs. Unexpectedly, our KcsA E71 A still showed, different from reports in the literature (Valiyaveetil et al., 2002), channel function with a high open probability in neutral DPhPC membranes. This agrees with previous observations for KcsA E71A (Rotem et al., 2010; McGuire \& Blunck, 2015) suggesting that the mutant KCSA E71A is sensitive to anionic lipids but that they are not mandatory for channel function. Addition of $\mathrm{Ba}^{2+}$ to the external buffer during channel measurements resulted in a strong reduction of the open probability, with no effect on the unitary single channel conductance (Winterstein et al., 2018, data not shown). This blocking effect has been reported from previous measurements of KCSA E71A after addition of $\mathrm{Ba}^{2+}$ (Banerjee \& Nimigean, 2011). It further indicates that KCSA E71 A expressed in the presence of nanodiscs and reconstituted into PLBss behaves in the same manner as reported in previous studies. 
To optimize channel insertion, nanodiscs should be adjusted to the POI. While the lipid composition of the nanodisc had no effect on channel properties, we found that for KCVNTS incorporation from POPC nanodiscs into the bilayer takes clearly longer than for DMPC and DMPG nanodiscs (fig. 10). From these experiments it cannot be concluded, how channel incorporation from a nanodisc into the target membrane takes place. One possibility would be for the channel to pass over from one membrane to the other, when the nanodisc is close enough to the target membrane. Another possibility is that the entire nanodisc incorporates into the target membrane before the scaffold protein opens up and releases the channel from the nanodisc lipid. The small amount of additional lipids from the nanodiscs is negligible compared to the large size of the target membrane. In the case of KcVNTS, DMPC nanodiscs seem to be the best choice for the most efficient incorporation. A possible explanation might be the thickness of the membrane. POPC bilayers are slightly thicker due to the longer fatty acids (C-16 and C-18) compared to DMPC/DMPG (C-14). KCVNTS is already completely embedded into DMPC membranes (Braun et al., 2014), the slight increase in thickness might be sufficient to cause a slower transition from nanodisc into the target bilayer. It remains to be reviewed if this is a general phenomenon or channel-specific.

The present data show that also the synthetic channel BLINKI could be successfully investigated on the single channel level using in vitro protein expression in the presence of nanodiscs (MM). In DPhPC membranes, single channel currents with the same unitary single channel conductance as KCVPBCV-1 could be measured (fig. 11 B). Two different gating types could be seen, concerning the open probability. Type 2 was identical to the wild type channel. Type 1 on the other hand has not only an increased open probability, but also an inverse voltage dependency: here the open probability increases up to $\sim 20 \%$ with positive voltages (fig. $11 \mathrm{C}$ ). The appearance of an additional gating type must be due to the extension of the $\mathrm{N}$-terminus. Previous studies have shown that the interaction between the $\mathrm{N}$ - and $\mathrm{C}$-terminus of $\mathrm{KCV}_{\mathrm{PBCV}-1}$ plays an important role in channel gating, due to formation of salt bridges between the free negative charge of the last amino acid at the C-terminus (leucine 94) and the positively charged amino acids within the short helix of the N-terminus (Hertel et al., 2010; Tayefeh et al., 2009). Rearrangement of the $\mathrm{N}$-terminus might lead to an altered conformation, favoring the open state. In chapter 3.2, gating properties of KCVPBCV-1 and changes at position 94 are examined in more detail.

While BLINKI could be characterized on the single channel level, no differences in gating could be observed upon transition from darkness to blue light (fig. 12). This result may not be too surprising. BLINKI was designed and optimized for function in living cells. The in vitro expression system may lack essential enzymes that contribute to a successful light sensitivity. One important component is riboflavin. It was added to the protein expression to provide the cofactor flavin mononucleotide, which is essential for subsequent blue light detection by binding to the LOV2 domain (Christie, 2007). For improvement of the light sensitivity of BLINK1, a myristoylation/palmitoylation sequence was inserted in the channel sequence (Cosentino et al., 2014). The required enzymes for membrane coupling are not included in the in vitro expression kit, so post-translational modification and consequential attachment of the protein to the membrane may not have taken place. Since protein expression into nanodiscs takes place in vitro, posttranslational coupling to the target membrane cannot be accomplished, which might explain the loss of sensitivity towards blue light. As mentioned before, BLINK1 is the predecessor of BLINK2 (Alberio et al., 
2018). While BLINK1 provides a lot of favorable properties, it has a very low surface expression. For improvement, the C-terminus of KATI was added to the C-terminus of BLINK1 to promote forward trafficking. The resulting channel BLINK2 is still 100\% regulated by light, but with an improved expression efficiency from $8 \%$ to $28 \%$. Single channel measurements have shown that the single channel conductance of BLINK2 is still identical to those of BLINK1 obtained from PLB measurements (fig. 13, Alberio et al., 2018).

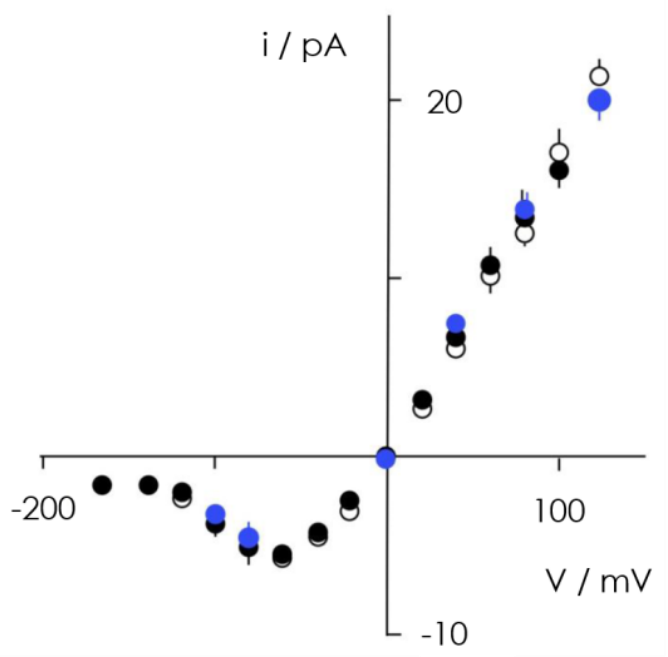

Figure 13 - i/V curves of BLINK1, BLINK2 and KCVPBCV-1. Comparison of the unitary single channel conductances of BLINK1 (white circles), BLINK2 (blue circles) and KCVPBCV-1 (black circles). Data are mean values standard deviation for $n$ independent measurements ( $n=3$ for all channels). Measurements of BLINKI and KCVPBCV-1 were performed after insertion of purified proteins in PLBS (symmetrical conditions with $100 \mathrm{mM} \mathrm{KCl}, 10 \mathrm{mM}$ HEPES at $\mathrm{pH}$ 7), while BLINK2 was measured with the patch clamp method in the cell-attached configuration $\left(103 \mathrm{mM} \mathrm{K}{ }^{+}\right.$in the pipette solution. From Alberio et al. (2018).

\subsubsection{Conclusion}

In summary, the PLB method is an ideal approach for investigating proteins in a reduced and controlled environment. In vitro protein expression in the presence of nanodics turns out to be an uncomplicated, fast and contamination-free way of receiving the POI in less than a few hours. However, this method is not suitable for the investigation of proteins that undergo post-translational changes. The reduced expression system lacks enzymes required for post-translational modification. The results of these experiments have proven that the target membrane properties determine channel function, so any commercially available nanodisc can be used to add to the expression lysate. It is an ideal method to investigate structure/function correlations of membrane proteins. 


\subsection{Mutation of leucine 94 leads to changes in gating of KCVPBCV-1}

An important reason for working with viral $\mathrm{K}^{+}$channels is their small size. $\mathrm{KCV}_{\mathrm{PBCV}-1}$ is composed of only 94 aa per subunit and is with this low number one of the smallest known $\mathrm{K}^{+}$channels. It has been mentioned before that the channel only consists of two TM helices; they are linked by 44 aa which contain the SF. The channel possesses a 12 a a N-terminal helix and lacks a cytoplasmic C-terminus. Despite its minimalistic design, $\mathrm{KCV}_{\mathrm{PBCV} \text {-1 }}$ still exhibits distinct gating characteristics. As has been previously described (Abenavoli et al., 2009) and can also be seen in fig. 14, gating of KCVPBCV-1 is characterized by both, fast gating at high negative potentials (so-called flicker gating) and a voltage dependency of the open probability, resembled by a slight activation with decreasing voltages. This is surprising, since the channel neither possesses the bundle crossing of the second TM helices (Tayefeh et al., 2007), which is known to be responsible for gating in other $\mathrm{K}^{+}$channels such as KcsA (Cuello et al., 1998), nor a voltage sensing domain as known for voltage-dependent $\mathrm{K}^{+}$channels (Tempel et al., 1987; Aggarwal \& MacKinnon, 1996). For this combination of structural simplicity and functional complexity, KCVPBCV-1 is known to be a prominent and highly suitable model system for better understanding the structural and functional properties which make such a small channel work in the same way as significantly bigger and more complex $\mathrm{K}^{+}$channels. Previous studies have shown that there are two possible gates in KCVPBCV-1. The first one is associated with the SF and responsible for the fast gating (flicker gating) of the channel which occurs at high negative voltages (Abenavoli et al., 2009). The second one involves the formation of salt bridges between the free negatively charged carboxyl group of the C-terminus and the positively charged amino acids within the 12 a long slide helix of the N-terminus (Moroni et al., 2002; Hertel et al., 2010). In the work of Moroni et al., 2002 it is shown that a truncated form of KCVPBCV-1, which lacks the N-terminus, is non-functional.

Further investigations of this issue (Hertel et al., 2010; Tayefeh et al., 2009) have additionally shown that the crucial parts of the N-terminal helix are the positively charged aa, which are responsible for the creation of the gate-forming salt bridges. The most stable salt bridge forms between lysine at position 6 and the free carboxyl group of the last amino acid, which is leucine at position 94 (Hertel et al., 2010). While a stable salt bridge is formed, there is no free charge at the entrance to the cavity, allowing $\mathrm{K}^{+}$ions to enter or leave freely. By disruption of the salt bridge, the carboxyl group is freely available, with an exposed negative charge. In this case free $\mathrm{K}^{+}$ions can bind to the four free negative charges of leucine 94 , blocking the entrance or exit of the cavity for further $\mathrm{K}^{+}$ions. The constant formation and disruption of these salt bridges determines part of the gating of the channel (Hertel et al., 2010).

In the work of Gebhardt (2010), position 94 in KCVPBCV-1 was already extensively investigated. Experimental and computational data was compared to determine interactions of the C-terminus with the rest of the channel. Yeast growth assays were combined with alanine-scanning mutagenesis of both TM helices, which revealed that leucine 94 plays a crucial role in channel function. By mutation of leucine at position 94, the free carboxyl group is still present, so only the influence of side chain properties can be investigated. Concurrently, the formation of salt bridges should still be possible. For this technique, a yeast strain lacking an endogenous $\mathrm{K}^{+}$-uptake system (Chatelain et al., 2009) was exposed to media with different $\mathrm{K}^{+}$concentrations. The strain was not able to survive on low $\mathrm{K}^{+}$concentrations, unless it was 
previously transfected with a functional $\mathrm{K}^{+}$channel, in this case KCVPBCV-1. Mutation of leucine 94 to alanine reduced yeast growth compared to the wild type. This indicated that there must be another mechanism besides the formation of salt bridges at the C-terminus which contributes to channel gating. Based on this result, leucine 94 was mutated to all other 19 proteinogenic amino acids of the standard genetic code. For all possible mutants, $\mathrm{K}^{+}$concentration profiles along the channel structure were calculated (Prof. Stefan Kast, TU Dortmund, Germany, Gebhardt, 2010) and yeast complementation assays were performed. Comparison of experimental and computational data showed that a lot of predicted results from the calculations could be confirmed by analyses of the experimental results, based on the yeast complementation assays. Yet some results from the experiments did not meet the expectations or contradicted the computational data (Gebhardt, 2010). Yeast growth assays are a highly suitable and fast method to get preliminary information about channel function. It is worth noting that the yeast complementation assay is a rather indirect method for testing channel function. The assay interprets the ability of growth rescue in relation to the function of the channel of interest. Other parameters, such as channel expression or sorting which could be affected by a mutation and in this way alter the ability to rescue yeast growth, are ignored.

Furthermore, the yeast assay does not provide information on the level of the single channel. Hence to understand the effect of a mutation at position 94 of $\mathrm{KCV}_{\mathrm{PBCV}-1}$ and to further help improve the possibility to predict channel behavior via computational analyses, KCVPBCV-1 L94 mutants were in this study characterized on the single channel level with the help of the PLB method. This method provides all the essential information required for a detailed structure/function correlation. It is also interesting to see to what extend the results of these two methods are comparable.

\subsubsection{KCVPBC-1 in planar lipid bilayers}

To examine the influence of mutations of the C-terminal aa L94 on KCVPBCV-1 gating on the single channel level, the wild type channel needs to be characterized first. For this, the channel was expressed in vitro in the presence of nanodiscs (Winterstein et al., 2018). All functional measurements were performed using the PLB method under symmetrical conditions (100 mM KCl, $10 \mathrm{mM} \mathrm{HEPES,} \mathrm{pH} \mathrm{7).} \mathrm{Artificial} \mathrm{membranes}$ were exclusively made of DPhPC in all experiments. Voltage protocols were applied from $-160 \mathrm{mV}$ to +160 $\mathrm{mV}$ in $20 \mathrm{mV}$ steps for $5 \mathrm{~min}$ per voltage.

Fig. 14 A shows representative single channel traces of KCVPBCV-1 at $\pm 120 \mathrm{mV}$. Two different levels can be defined as the open (O) and closed (c) state. The traces indicate that the channel has an altogether very low open probability and a much higher conductance at positive than at negative voltages. At positive voltages, gating is defined by high-conducting open states with very short open dwell times, interrupted by closed states of short and medium length. With increasing negative voltages, gating is more defined by longer closed states, interrupted by so-called burst events (Sakmann et al., 1980). During these bursts, the dwell times of the closed states are much shorter, while the dwell times of the open states are longer compared to those at positive voltages, but also of much lower conductivity. These observations can be confirmed by looking at the corresponding i/V- (fig. 14 B) and open probability curves (fig. 14 C). In the 
range of $+160 \mathrm{mV}$ to $0 \mathrm{mV}$ an ohmic conductivity can be seen. The slope of the i/ $\mathrm{V}$ curve in the linear range, indicated by the red dashed line, represents the single channel conductance $(\gamma)$ and was calculated with equation (1). For KCVPBCV-1 the calculated unitary single channel conductance in buffer solutions containing $100 \mathrm{mM} \mathrm{KCl} / \mathrm{pH} 7$ in the range of $0 \mathrm{mV}$ and $+160 \mathrm{mV}$ is $174 \pm 9 \mathrm{pS}$. This value agrees well with the data from the literature (Pagliuca et al., 2007). With increasing negative voltages, a decrease of the current amplitude occurs, resulting in a negative slope conductance. This effect was already described by Schroeder \& Hansen $(2006,2007)$ as flicker gating. It is characteristic for Kcv channels and has been previously described for $\mathrm{KCV}_{\mathrm{PBCV} \text {-1 }}$ from patch clamp recordings in Xenopus oocytes (Abenavoli et al., 2009) and PLB measurements in DPhPC (Shim et al., 2007). Flicker gating is due to the very fast gating of the channels, which cannot be entirely resolved by the measuring device, resulting in a shift of the current distribution towards zero. The true current could be deducted with beta fit analysis and was shown to follow the linear extrapolation of the ohmic conductance seen in the range from $0 \mathrm{mV}$ to -40 $\mathrm{mV}$ (Abenavoli et al., 2009; Schroeder, 2015).

As indicated by the single channel traces, the open probability of KCVPBCV-1 (fig. $14 \mathrm{C}$ ) is very low (1-5\%). Within this low open probability, a slight voltage dependency can be seen, appearing as a minimal, but steady increase from a value below $1 \%$ at positive voltages to about $5 \%$ at high negative voltages. This voltage dependency results from the increasing dwell times of the open states with negative voltages. 


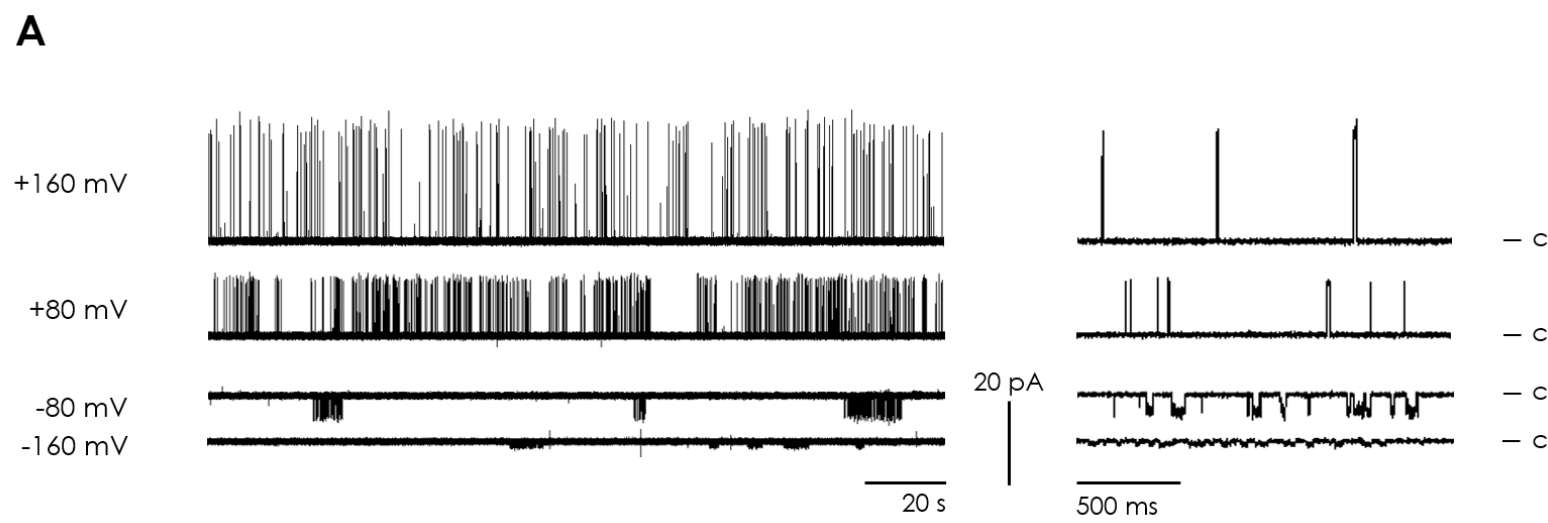

B

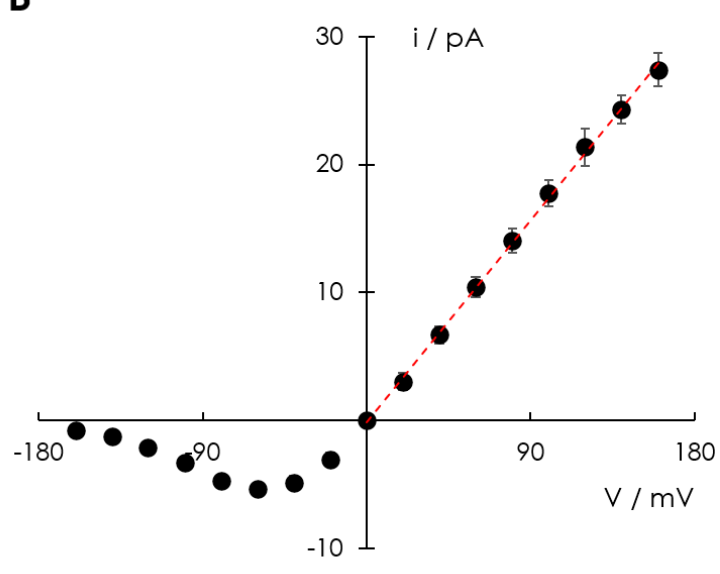

C

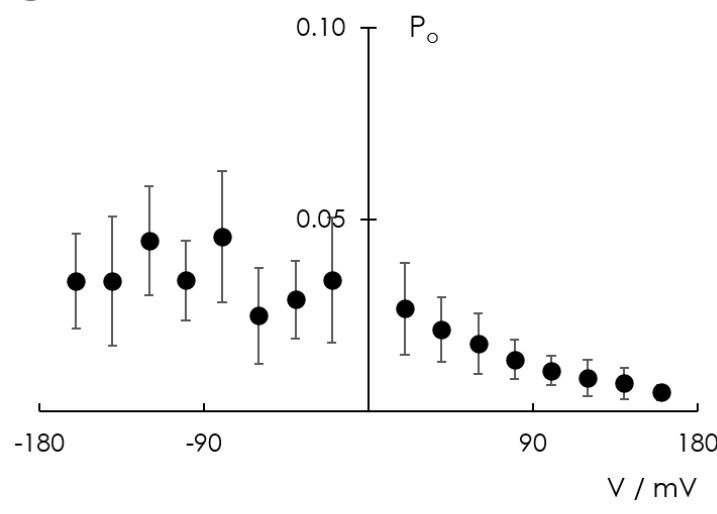

Figure 14 - Single channel properties of KCVPBCV-1. (A) Representative single channel traces of KCVPBCV-1 from $+160 \mathrm{mV}$ to $-160 \mathrm{mV}$ in $80 \mathrm{mV}$ steps with two different temporal resolutions. Traces with higher temporal resolution (right side) show individual gating events in detail and do not reflect the general open probability. $\mathrm{c}$ marks the closed state. For better visualization, data was subsequently filtered with $500 \mathrm{~Hz}$. (B) I/V curve for KCVPBCV-1. The red dashed line indicates the unitary single channel conductance in the voltage range between $0 \mathrm{mV}$ to $+160 \mathrm{mV}$. (C) Open probability curve for $\mathrm{KCV}_{\mathrm{PBCV}-1}$. Data are mean values \pm standard deviation for $\mathrm{n}$ independent measurements $(\mathrm{n}=8)$. Measurements were performed under symmetrical conditions with $100 \mathrm{mM} \mathrm{KCl}$ and $10 \mathrm{mM}$ HEPES at $\mathrm{pH} 7$. 


\subsubsection{Mutation of leucine 94}

To examine the influence of position 94 in $\mathrm{KCV}_{\mathrm{PBCV}-1}$, the naturally occurring leucine was replaced by all other 19 proteinogenic amino acids of the standard genetic code. All 19 mutants KCVPBCV-I L94X (wherein $X$ represents the corresponding mutated amino acid) were examined on the single channel level with the PLB method for differences in open probability and unitary single channel conductance. All measurements were performed as in fig. 14 under symmetrical conditions, with $100 \mathrm{mM} \mathrm{KCl}$ and $10 \mathrm{mM}$ HEPES at pH 7. Artificial membranes were exclusively made of DPhPC. Clamp protocols were applied from $-160 \mathrm{mV}$ to $+160 \mathrm{mV}$ in $20 \mathrm{mV}$ steps for $5 \mathrm{~min}$ per voltage, due to the low open probability.

Different analyses were considered for comparison of the open probability of $K_{C V_{P B C V}-1}$ and $K_{C V} V_{P B C V}-1$ L94X. Firstly, the mean open probability from $-160 \mathrm{mV}$ to $+160 \mathrm{mV}$ was calculated and compared, as shown in fig. 15 A. These results give a first indication for mutation generated differences in the mean open probability compared to the wild type. The red dotted line indicates the level of the wild type. The colors of the bars have been adjusted to amino acid properties: green for polar/neutral, yellow for unipolar/hydrophobic, blue for basic and red for acidic amino acids. The majority of mutations lead to a decrease of the already very low mean open probability of KCVPBCV-1 (M, D, A, W, S, N, F, G, V) or have no visible effect $(Y, T, H, E, Q, R, K, I)$. Only mutations of $L 94$ to proline $(P)$ and cysteine $(C)$ influence the mean open probability, causing a definite increase. However, this crude analysis does not allow any interpretations about changes in the voltage dependency. Therefor secondly an additional analysis was applied, calculating the difference between the open probability value at -160 $\mathrm{mV}$ and $+160 \mathrm{mV}$ for each mutant and comparing the results to the wild type channel, shown in fig. $15 \mathrm{~B}$. It was already mentioned before that KCVPBCV-1 shows a slight voltage dependency, emerging as an increase of the open probability with values at around $1 \%$ at positive voltages up to values around $5 \%$ with more negative voltages (fig. $14 \mathrm{C}$ ). The red dotted line again indicates the wild type level. Mutants with positive values above this level have an increased (C, P), below this level a decreased voltage dependency (T, V, A, N, $S, M, D)$, compared to $K_{C V_{P B C V}-1}$. Negative values of the difference in open probability from - 160 and +160 $\mathrm{mV}$ correspond to a reversed voltage dependency, which can be seen for L94R and L94K. This second analysis gives additional information on the mean open probability: The decrease of the mean open probability for L94M, L94D, L94A, L94S, L94N and L94V can be explained by a loss of the voltage dependency after mutation. L94W, L94F and L94G have a lower mean open probability than KCVPBCV-1 but have not completely lost their voltage dependency. L94T has a mean open probability only slightly reduced compared to the wild type, with an also slight loss of voltage dependency. L94Y, L94H, L94E, L94Q and L94I are in the same range as KCVPBCV-1, for both mean open probability and voltage dependency. For L94R and L94K, the mean open probability is in the same range as the wild type. Fig. 15 $B$ additionally shows that the values for the difference in open probability between -160 and $+160 \mathrm{mV}$ for these two mutations are negative, corresponding to a reversed voltage dependency. Here the open probability increases with more positive voltages. Two mutations, L94P and L94C, lead to a considerable increase in mean open probability and voltage dependency, which will be viewed in more detail in chapters 3.2.3 and 3.2.4. Color coding the results with corresponding amino acid properties show that no obvious connections between the single groups can be seen, except for the reversed voltage 
dependency. This appears after mutating leucine 94 to arginine and lysine, which both possess a basic side chain. What is unexpected, however, is that $\mathrm{L} 94 \mathrm{H}$ shows nearly the same voltage dependency as the wild type, despite the also basic side chain of histidine. One reason for this could be the different pKa values of the amino acids within the protein. Since the amino acid at position 94 is exposed to the medium, the proton concentration within the buffer solution determines whether this amino acid is in its protonated or deprotonated state. This could correspond to different protein conformations and consequential differences of the channels gating properties. The effect of proton concentration within the measuring solution will be examined in more detail in chapter 3.2.6. Since no other commonalities could be seen by categorizing the amino acids by their properties, two additional factors were considered: hydrophobicity (fig. $15 \mathrm{C}$ ) and molecular weight (fig. $15 \mathrm{D}$ ). For the correct formation of the secondary structure, the hydrophobicity of interacting amino acids is important (Ide et al., 1997). Kyte \& Doolittle (1982) classified the side chains of amino acids based on their hydrophobicity. Fig. $15 \mathrm{C}$ shows the mean open probability of KCVPBCV-1 and KCVPBCV-1 L94X in relation to these hydrophobicity values. Individual data points are shown by using the single letter abbreviation for the corresponding amino acid. For better comparison, the wild type $(\mathrm{L})$ is shown in red. The figures shows that there is no relation between hydrophobicity and changes in the open probability. Cysteine and proline have different values, yet both lead to an increase in the mean open probability. Amino acids that lie between the area of the hydrophobicity values of cysteine and proline do not have this effect.

To see if the size of the inserted amino acid relates to changes in the mean open probability, fig. $15 \mathrm{D}$ shows the relation between the mean open probability and the molecular weight in the same way as shown in fig. $15 \mathrm{C}$. There is also no relation seen depending on the size. As for the hydrophobicity, cysteine and proline stand out incoherently. 

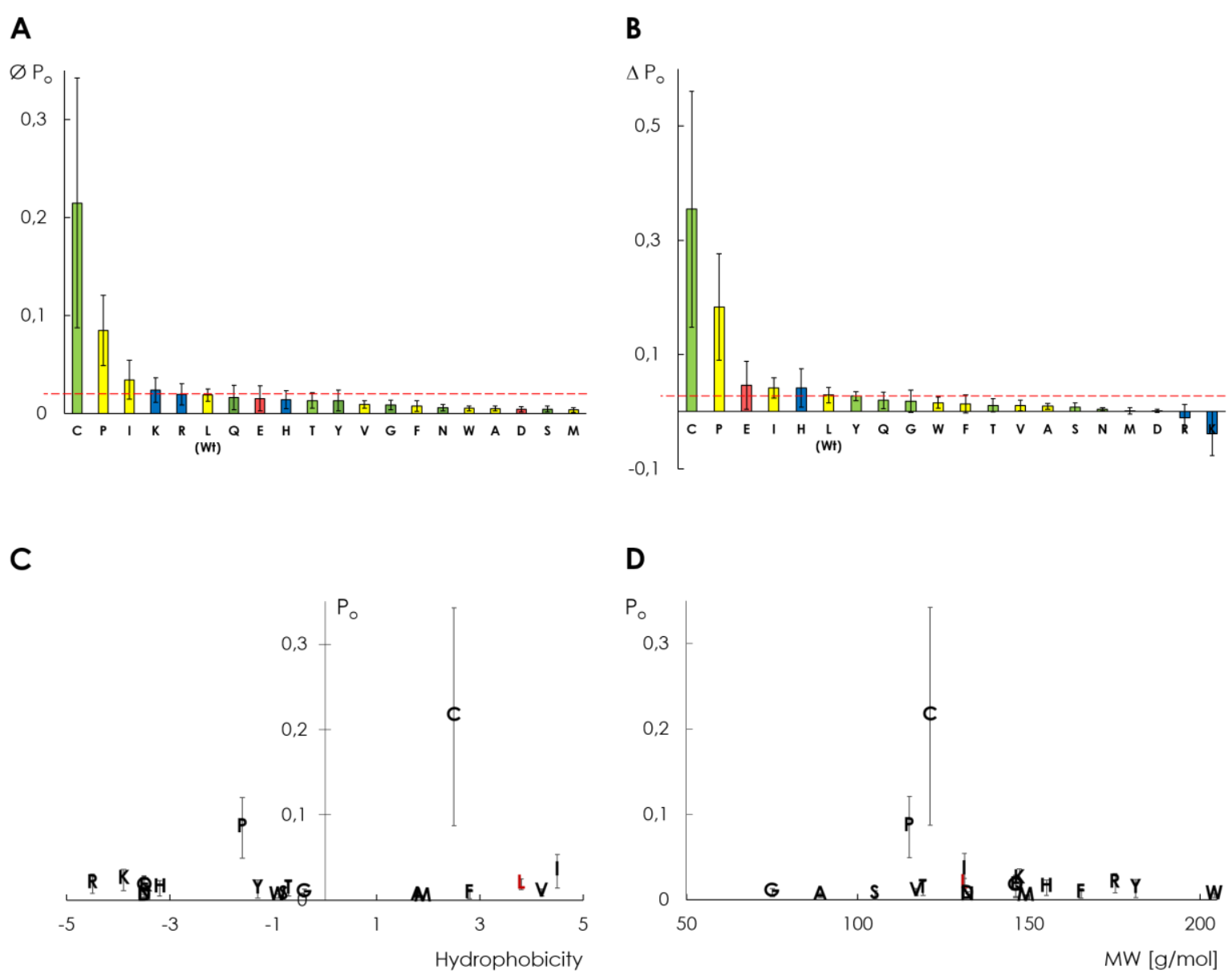

Figure 15 - Open probability of $\mathrm{KCV}_{\mathrm{PBCV}-1}$ and $\mathrm{KCV}_{\mathrm{PBCV}} \mathrm{L} \mathrm{L94X}$ as a function of physicochemical properties of the last amino acid. (A) Mean open probability for KCVPBCV-1 and KCVPBCV-1 L94X in the range of 160 to $+160 \mathrm{mV}$. (B) Difference between the Po value at $-160 \mathrm{mV}$ and $+160 \mathrm{mV}$ for KCVPBCV-1 and $\mathrm{KCV}_{\mathrm{PBCV}-1} \mathrm{L94X}$. The $\mathrm{X}$ in KCVPBCV-1 L94X represents the mutation to different amino acids, abbreviated with the one letter code. Four different colors stand for four different amino acid properties: green for polar/neutral, yellow for unipolar/hydrophobic, blue for basic and red for acidic amino acids. The level of the wild type (L) KCVPBCV-1 is marked with red dotted lines. (C) Open probability plotted against the hydropathy index from Kyte and Doolittle (1982). (D) Open probability plotted against the molecular weight of the individual amino acids. Data points are represented by the corresponding one letter abbreviation for the respective amino acid. The wild type is additionally marked in red. Data are mean values \pm standard deviation of $n$ independent measurements ( $A$ : $n=3, C: n=6, D: n=4, E: n=7, F: n=6, G: n=4, H: n=3, I: n=4, K: n=3, L(W+): n=8, M: n=3, N: n=4, P: n=3$, $Q: n=3, R: n=3, S: n=3, T: n=3, V: n=4, W: n=3, Y: n=4)$, performed under symmetrical conditions with $100 \mathrm{mM} \mathrm{KCl}$ and $10 \mathrm{mM}$ HEPES at $\mathrm{pH} 7$. 
The second functional property of the channel that was compared is changes in the unitary single channel conductance of KCVPBCV-1 caused by mutation of leucine at position 94. As described in chapter 3.2.1 for the wild type channel, the ohmic conductance was calculated with equation (1) between 0 and $+160 \mathrm{mV}$. Fig. 16 shows an overview of the unitary single channel conductance of KCVPBCV-1 and KCVPBCV-1 L94X. The red dashed line again indicates the level of the wild type channel and the colors of the bars have again been adjusted to amino acid properties: green for polar/neutral, yellow for unipolar/hydrophobic, blue for basic and red for acidic amino acids. For most of the mutants, no significant difference or only a slight increase or decrease in conductance can be observed (E, T, D, F, V, Y, W, I, A, N, M, S, Q, C, G). Four mutants, L94P, L94H, L94K and L94R show a considerable decrease in their single channel conductance. The effect of L94P is shown in more detail in chapter 3.2.3. The commonality of three of these amino acids $(H, K, R)$ is the basic side chain and consequential free electron pairs of nitrogen in their side chain, which develop a positive charge after proton uptake (Katakuse et al., 1979). Interestingly, mutation of L94 to amino acids with acidic side chains slightly increases the unitary single channel conductance of KCVPBCV-1 $(174.4 \pm 9.1$ pS) by $6.4 \%$ for KCVPBCV-1 L94D to $185.5 \pm 7.5$ pS and by $10.2 \%$ for KCVPBCV-1 L94E to $192.1 \pm 14.4$ pS. The effect of proton concentration on single channel gating characteristics will be examined in more detail in 3.2.6.

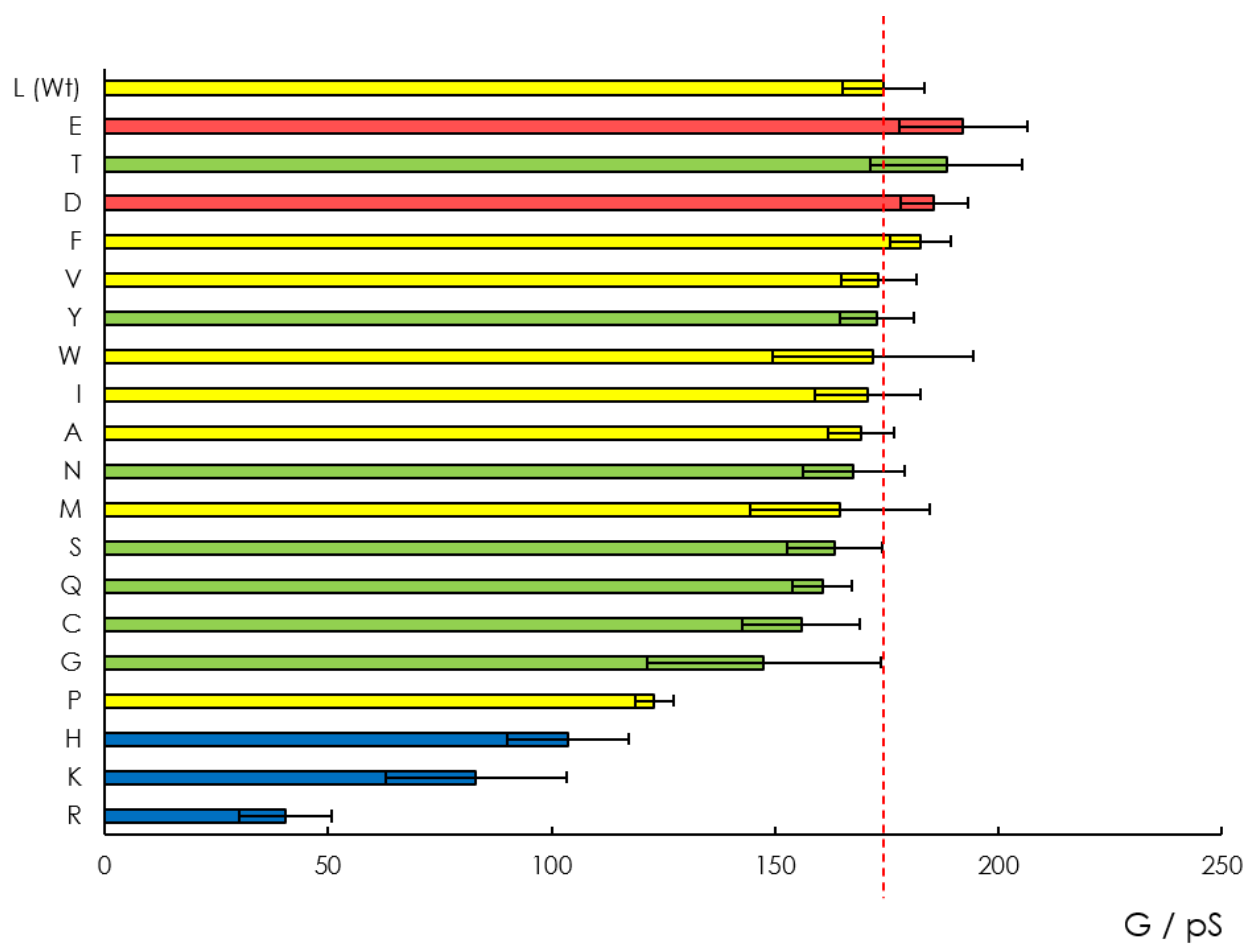

Figure 16 - Unitary single channel conductance of KCVPBCV-1 and KCVPBCV-1 L94X. Unitary single channel conductance for KCVPBCV-1 and KCVPBCV-1 L94X calculated between 0 and $+160 \mathrm{mV}$. Four different colors stand for four different amino acid properties: green for polar/neutral, yellow for unipolar/hydrophobic, blue for basic and red for acidic amino acids. The X in KCVPBCV-1 L94X represents the mutation to different amino acids, abbreviated with the one letter code. The level of the wild type (L) KCVPBCV-1 is marked with red dashed lines. Data are mean values \pm standard deviation of $n$ independent measurements $(A: n=3, C: n=6, D: n=4, E: n=7, F: n=6, G: n=4, H: n=3, I$ : $n=4, K: n=3, L(W t): n=8, M: n=3, N: n=4, P: n=3, Q: n=3, R: n=3, S: n=3, T: n=3, V: n=4, W: n=3, Y: n=4)$, performed under symmetrical conditions with $100 \mathrm{mM} \mathrm{KCl}$ and $10 \mathrm{mM} \mathrm{HEPES}$ at $\mathrm{pH} 7$. 
For completeness, the unitary single channel conductance of KCVPBCV-1 and KCVPBCV-1 L94X was plotted against the hydrophobicity values from Kyte \& Doolittle (1982, fig. 17 A) and molecular weight (fig. 17 B), as has already been done for the comparison of the open probability. There are again no apparent similarities of the mutated amino acids that cause a reduction of the unitary single channel conductance of $K_{C V P B C V-1}$. R, K, H and $P$ all possess negative hydrophobicity values, but the amino acids $D, E, N$ and $Q$ (all with the hydrophobicity value of -3.5 ) do not induce such an effect, on the contrary, mutation to $D$ and $E$ cause an increase of the conductance. There is also no apparent connection between molecular weight and impact on unitary single channel conductance. P, K, H and R stand out incoherently.

A

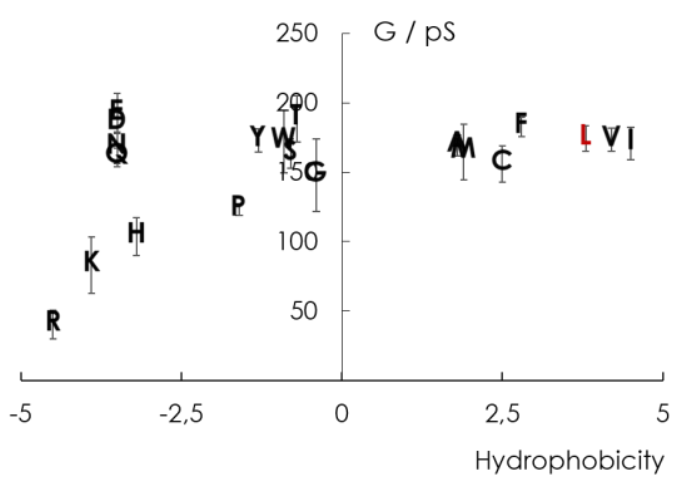

B

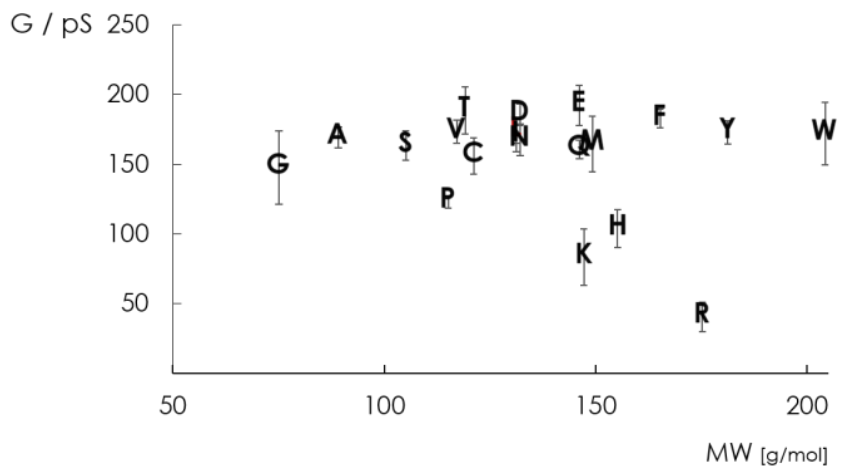

Figure 17 - Unitary single channel conductance of KCVPBCV-1 and KCVPBCV-1 L94X as a function of hydrophobicity and molecular weight. (A) Conductance plotted against the hydropathy index from Kyte \& Doolittle (1982) for KCVPBCV-1 and KCVPBCV-1 L94X. (B) Conductance plotted against the molecular weight of KCVPBCV-1 and KCVPBCV-1 L94X. X represents the mutation to different amino acids. Data points are represented by the corresponding one letter abbreviation for the respective amino acid. The wild type is additionally marked in red. Data are mean values \pm standard deviation of $n$ independent measurements ( $A: n=3, C: n=7, D: n=4, E: n=7, F: n=6, G: n=4, H: n=3, I: n=4, K: n=3, L$ $(W+): n=8, M: n=3, N: n=4, P: n=3, Q: n=3, R: n=3, S: n=3, T: n=3, V: n=4, W: n=3, Y: n=4)$, performed under symmetrical conditions with $100 \mathrm{mM} \mathrm{KCl}$ and $10 \mathrm{mM}$ HEPES at $\mathrm{pH} 7$. 


\subsubsection{KCVPBCV-1 L94P}

As mentioned in the previous chapter, mutation of leucine 94 in KCVPBCV-1 to proline leads to a change channel function for both parameters, unitary single channel conductance and open probability. Fig. 18 A shows representative single channel traces of a measurement at $+120 \mathrm{mV}$ and $-120 \mathrm{mV}$ for a $\mathrm{K}^{+}$ concentration of $100 \mathrm{mM}$ at $\mathrm{pH}$ 7. The voltage dependency is still present, for open events at positive voltages are less and shorter than at negative voltages. A section of the measurement at $+120 \mathrm{mV}$ with a higher time resolution (fig. $18 \mathrm{~A}$, right side) shows that for short periods the channel switches between two levels with different conductivities. Fig. 18 B shows these two different levels (light and dark red) compared to the wild type KCVPBCV-1 (black). The main conductance, which is listed in fig. 17, has a much higher open probability and is clearly reduced compared to the wild type. For short dwell times the channel reaches a higher conducting state (fig. 18 A). Plotted, this higher conductance lies in the same range as the conductance of $\mathrm{KCV}_{\mathrm{PBCV}-1}$. These different states can only be seen at positive voltages. At negative voltages, no second level can be identified and the conductance is unaltered to that of

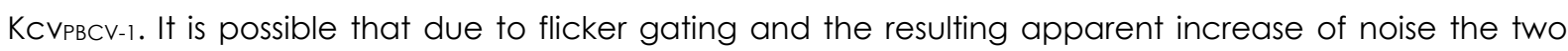
different states cannot be distinguished from each other. Another possibility is that this effect is only present at positive voltages. The main and lower conductance has a higher open probability and was considered for analysis (fig. $18 \mathrm{C}$ ). While at positive voltages there is no considerable difference in the open probability compared to the wild type, the voltage dependency for KCVPBCV-1 L94P has strongly increased, leading to much higher values of up to $20 \%$ at negative voltages. This increase of both, mean open probability (fig. 15 A) and voltage dependency (fig. 15 B) for the mutant KCVPBCV-1 L94P is due to the increase of dwell times of the open events at negative voltages. 


\section{A Kcv L94P}<smiles>CC(=O)C1CCCN1C(C)=O</smiles><smiles>C1=C[CH-]1</smiles><smiles>CC(=O)C1CCCN1C(C)=O</smiles>

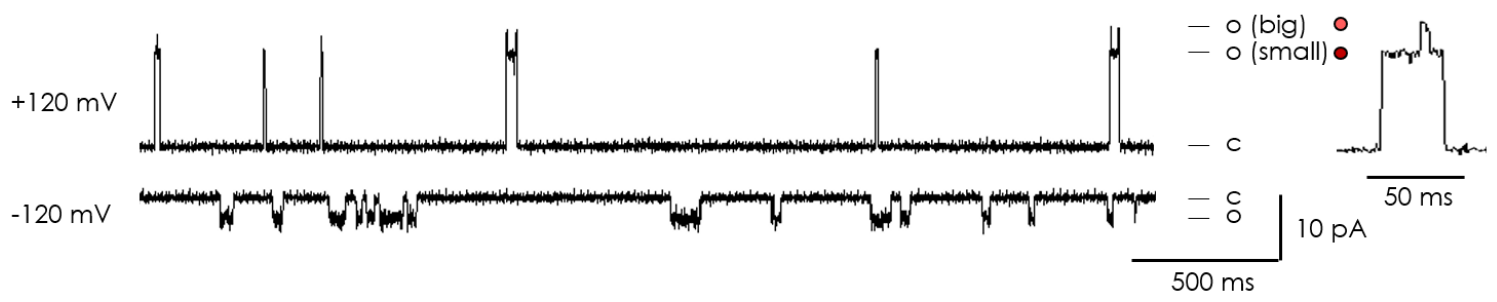

B

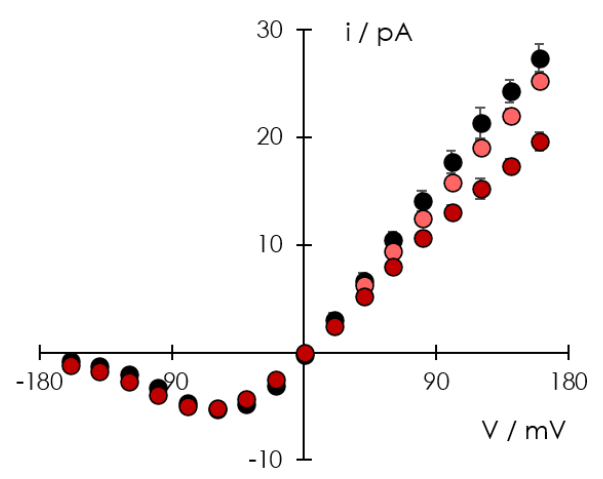

C

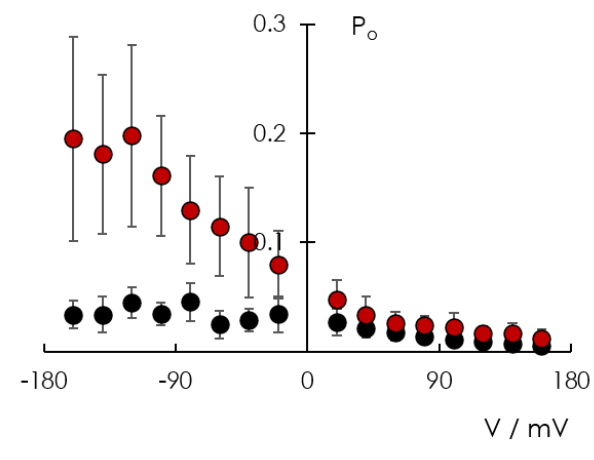

Figure 18 - Single channel properties of KCVPBCV-1 L94P. (A) Chemical structure of proline (†op) in its trans and cis conformation. Representative single channel traces of KCVPBCV-1 L94P at $+120 \mathrm{mV}$ (top) and $-120 \mathrm{mV}$ (bottom). $\mathrm{c}$ and o mark the closed an open state. For better visualization, data was subsequently filtered with $500 \mathrm{~Hz}$. (B) I/V curves for KCVPBCV-1 (black), the low conductance (dark red) and high conductance (light red) of KCVPBCV-1 L94P. (C) Open probability curves for KCVPBCV-1 (black) and KCVPBCV-1 L94P (red). Data are mean values \pm standard deviation for $\mathrm{n}$ independent measurements (KCVPBCV-1: $n=8, K_{C V P B C V}-1$ L94P: $n=3$ ). Measurements were performed under symmetrical conditions with $100 \mathrm{mM} \mathrm{KCl}$ and $10 \mathrm{mM}$ HEPES at $\mathrm{pH} 7$. 


\subsubsection{KCVPBCV-1 L94C}

The second mutation that leads to an even stronger increase of the mean open probability compared to KCVPBCV-1 was the mutation of leucine 94 to cysteine. What stands out when looking at fig. $15 \mathrm{~A}$ and $\mathrm{B}$ is the size of the error bar for this mutant. Fig. 19 A shows two representative single channel traces from two different measurements of KCVPBCV-1 L94C. Both channels have the same single channel conductance but differ clearly in their open probability. Seven different measurements of KCVPBCV-1 L94C showed an even distribution of different open probabilities for the individual measurements, with values between $20 \%$ and $80 \%$ at high negative voltages, which cannot be divided into groups that would correspond to only two different gating modes. Despite the variety of gating types, there is still a clear increase of the mean open probability compared to $\mathrm{KCV}_{\mathrm{PBCV}-1}$, even for measurements with lower open probabilities. For better demonstration, the difference of the open probability between $-160 \mathrm{mV}$ and $+160 \mathrm{mV}$ is shown in fig. 20 for each individual measurement of KCVPBCV-1 L94C, compared to the Po difference of the wild type channel. Even for the smallest difference of $K_{C V B C V-1}$ L94C there is still a clear increase compared to KCVPBCV-1.

Mutation of leucine 94 to cysteine in KCVPBCV-1 only decreases the unitary single channel conductance

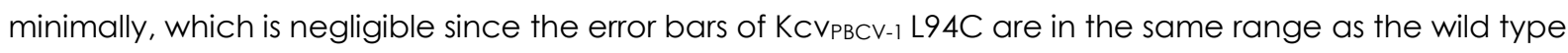
(fig. 19 B). The effect on the open probability is however distinct. While KCVPBCV-1 L94P only increases the open probability at negative voltages, mutation to cysteine increases the open probability over the entire voltage range. The effect intensifies with decreasing voltages (fig. $19 \mathrm{C}$ ).

An obvious assumption is that mutation to cysteine may lead to the formation of disulfate bridges between the mutated and another cysteine within the protein, stabilizing the open state. To test this hypothesis, measurements were performed before and after addition of dithiothreitol (DTT). DTT can reduce disulfide bonds within the protein (Konigsberg, 1972). Previous experiments with a different viral $\mathrm{K}^{+}$ channel, Kcvs, have shown that $5 \mathrm{mM}$ of DTT are sufficient to reduce possible disulfide bonds (Rauh et al., 2017). Since position 94 is at the $C$-terminal end of the protein and accessible from the intracellular side, 5 mM DTT was added only to the cis compartment of the chamber and carefully resuspended. Fig. 19 D\&E show the corresponding current-voltage relation and open probability of two measurements (green and blue) of KCVPBCV-1 L94C, before (circles) and after (triangles) addition of $5 \mathrm{mM}$ DTT to the cis compartment. Fig. $19 \mathrm{D}$ shows that even though the individual measurements have a slightly different unitary single channel conductance, after addition of $5 \mathrm{mM}$ DTT there is no difference in conductance for both measurements. The above-mentioned difference in gating types of KCVPBCV-1 L94C can be seen in fig. $19 \mathrm{E}$ : two measurements with very different but still high open probabilities. After addition of $5 \mathrm{mM}$ DTT, there is also no difference in the open probability. Minor differences between in the open probability before and after addition of DTT (for example at $+20 \mathrm{mV}$ and $-40 \mathrm{mV}$ ) are due to very long closed events that can still occur for KCVPBCV-1 L94C as they do for the wild type channel. This experiment shows that the changes in gating properties of KCVPBCV-1 after mutation of leucine 94 to cysteine are not due to the formation of disulfide bridges within the protein but must be caused differently. 
A $K c v L 94 C$<smiles>CC(N)C(=O)O</smiles>

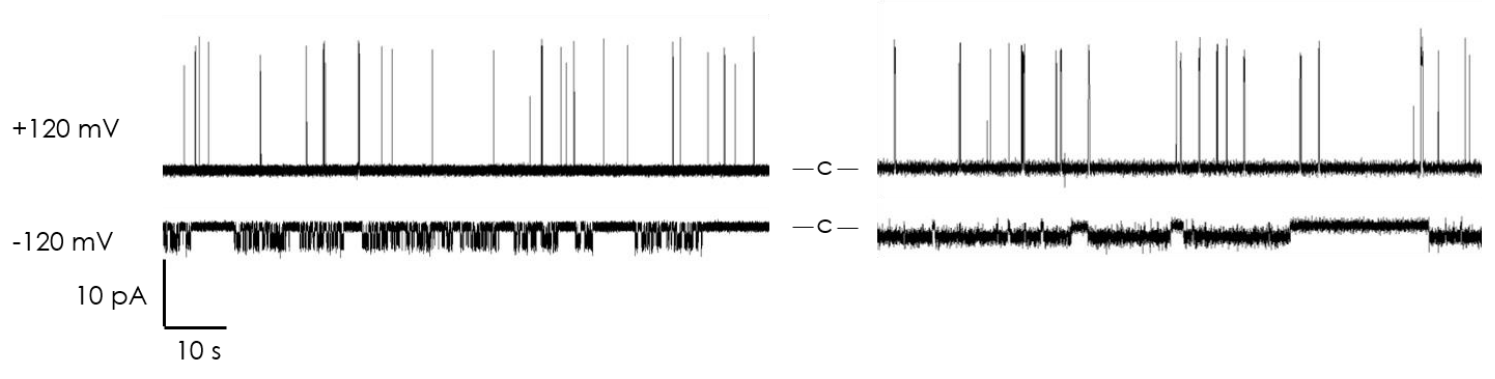

B

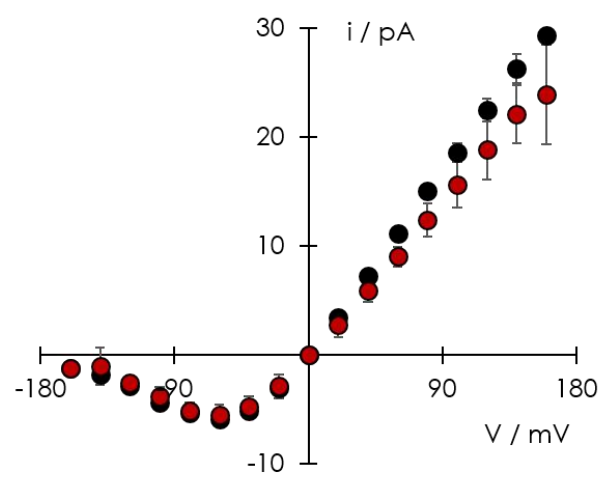

D

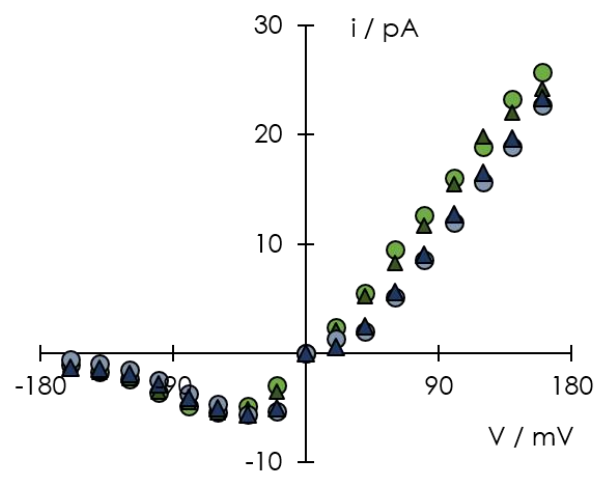

C

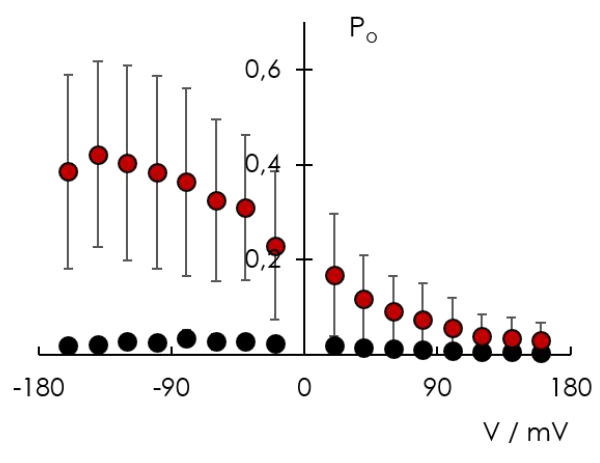

$\mathbf{E}$

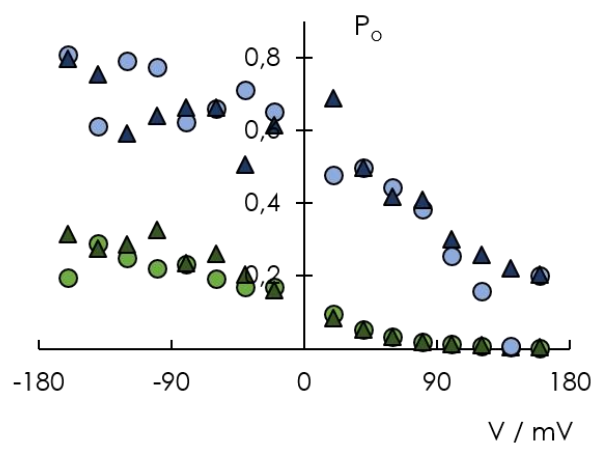

Figure 19 - Single channel properties of KCVPBCV-1 L94C. (A) Two different representative single channel traces of KCVPBCV-1 L94C at +120 mV (top) and -120 mV (bottom). c marks the closed state. For better visualization, data was subsequently filtered with $500 \mathrm{~Hz}$. (B) i/V curves for KCVPBCV-1 (black) and $\mathrm{KCV}_{\mathrm{PBCV}-1}$ L94C (red). (C) Open probability curves for KCVPBCV-1 (black) and KCVPBCV-1 L94C (red). Data are mean values \pm standard deviation for $n$ independent measurements (KCVPBCV-1: $n=8, K C V P B C V-1$ L94C: $n=6$ ) Measurements were performed under symmetrical conditions with $100 \mathrm{mM} \mathrm{KCl}$ and 10 mM HEPES at pH 7. (D) i/V curves for two different measurements of KCVPBCV-1 L94C (green and blue) before (circles) and after (triangles) addition of $5 \mathrm{mM}$ DTT. (E) Open probability curves for two different measurements of KCVPBCV-1 L94C (green and blue) before (circles) and after (triangles) addition of $5 \mathrm{mM}$ DTT. Measurements were performed under symmetrical conditions with $100 \mathrm{mM}$ $\mathrm{KCl}$ and $10 \mathrm{mM}$ HEPES at $\mathrm{pH} 7.5 \mathrm{mM}$ of DTT was added to the cis side of the chamber. 


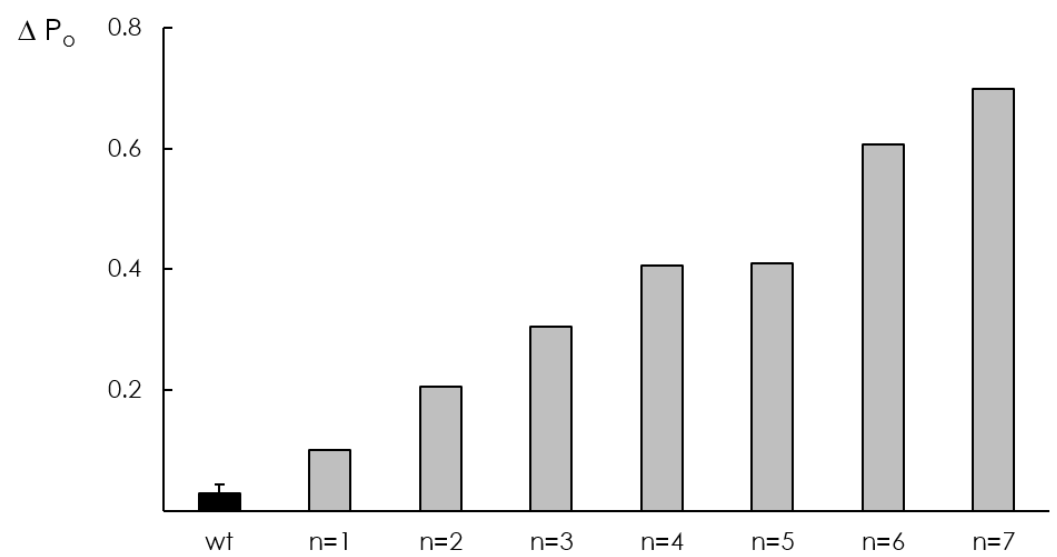

Figure 20 - Difference between the $\mathrm{P}_{\mathrm{o}}$ value at $-160 \mathrm{mV}$ and $+160 \mathrm{mV}$ for KCVPBCV-1 (wt) and KCVPBCV-1 $\mathrm{L}$ 94C. Mean value of KCVPBCV-1 (wt) in black with standard deviation of 8 independent measurements compared to the individual differences for each measurement independently for KCVPBCV-1 L94C in grey. Measurements were performed under symmetrical conditions with $100 \mathrm{mM} \mathrm{KCl}$ and $10 \mathrm{mM}$ HEPES at $\mathrm{pH} 7$. 


\subsubsection{KCVPBCV-1 L94H, KCVPBCV-1 L94K and KCVPBCV-1 L94R}

As mentioned in the previous chapter, the introduction of amino acids at position 94 with basic side chains seems to have multiple effects on single channel gating properties of KCVPBCV-1. All three mutations $L 94 \mathrm{H}$, L94K and L94R lower the unitary single channel conductance to a varying degree. Fig. 21 A shows exemplary extracts of open events for KCVPBCV-1, KCVPBCV-1 L94H, KCVPBCV-1 L94K and KCVPBCV-1 L94R at +120 $\mathrm{mV}$ and $-120 \mathrm{mV}$. These sections do not represent the general open probability of the respective channels but show individual gating events for each channel in more detail, respectively. KCVPBCV-1 L94H is characterized by open events with short dwell times at positive voltages, which increase with lower voltages, similar to the wild type but with a reduced single channel conductance. Additionally, bursts of activity with no defined conductance level can appear, as shown in the red dashed box. These burst events are only seen at positive voltages. The unitary single channel conductance of KCVPBCV-I L94K is further reduced. For this mutant the dwell times of the open events have changed. They are a lot higher at positive voltages and decreasing with more negative voltages. While the dwell times of open events at positive voltages are longer, they are separated by very long closed events, in the range of several seconds. The most significant reduction of unitary single channel conductance can be seen for KCVPBCV-1 L94R. With only $40.4 \pm 10.4$ PS it is only about $23 \%$ of the conductance of the wild type. Open events can be slightly longer at positive voltages compared to KCVPBCV-1 but are not separated by very long closed events. Dwell times of open events at negative voltages are slightly shorter and less frequent. These observations are confirmed by the corresponding current-voltage (fig. 21 B) and open probability (fig. 21 C) curves. Fig. $21 \mathrm{C}$ also shows the reversed voltage dependency of the open probability for KCVPBCV-1 L94K and KCVPBCV-1 L94R, which has been described previously in chapter 3.2.2 (fig. 15 B). From the single channel traces it becomes clear that the reversed voltage dependency is due to the higher dwell times of open events at positive voltages which become shorter with decreasing voltages, in the case of KCVPBCV-1 L94K. The dwell times of open events for KCVPBCV-1 L94R at positive voltages only increases slightly but the number of open events is higher, which causes the general open probability to rise. The number of events decreases with lower voltages. There is no change in the mean open probability of voltage dependency for $\mathrm{KCV}_{\mathrm{PBCV}-1} \mathrm{~L} \mathrm{~L} 4 \mathrm{H}$, it is nearly in the same range as for $\mathrm{KCV}_{\mathrm{PBCV}-1}$. One plausible explanation for this phenomenon is the possibility of histidine to be in either a protonated or deprotonated state, depending on the pKa value of the amino acid within the protein. Since the latter cannot be determined due to its variability within a protein (Edgcomb \& Murphy, 2002), it cannot be defined if histidine $94 \mathrm{at} p \mathrm{pH}$ 7 exists in its protonated or deprotonated state. To examine the influence of proton concentration on $\mathrm{KCV}_{\mathrm{PBCV}-1}$ and KCVPBCV-1 $\mathrm{L94H}$, further experiments were performed with varying $\mathrm{pH}$ values. 
A

$+120 \mathrm{mV}$

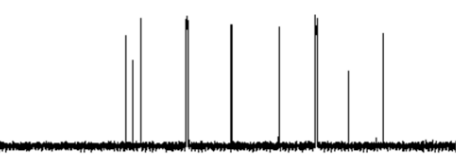

$\mathrm{KCV}_{\mathrm{PBCV}-1} \mathrm{~L} \mathrm{~L} 4 \mathrm{H}$

$\mathrm{KCV}_{\mathrm{PBCV}-1} \mathrm{~L}$ (94K

$\mathrm{KCV}_{\mathrm{PBCV}-1} \mathrm{L94R}$
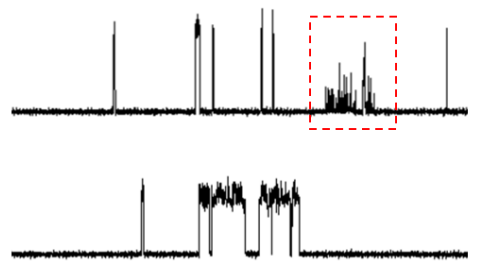
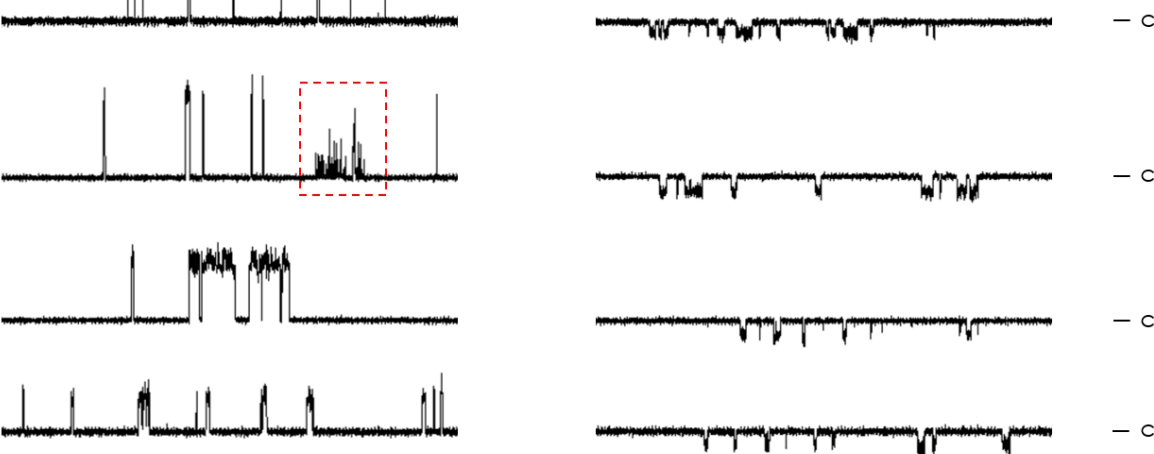

$\left.10 \mathrm{pA}\right|_{500 \mathrm{~ms}}$

B

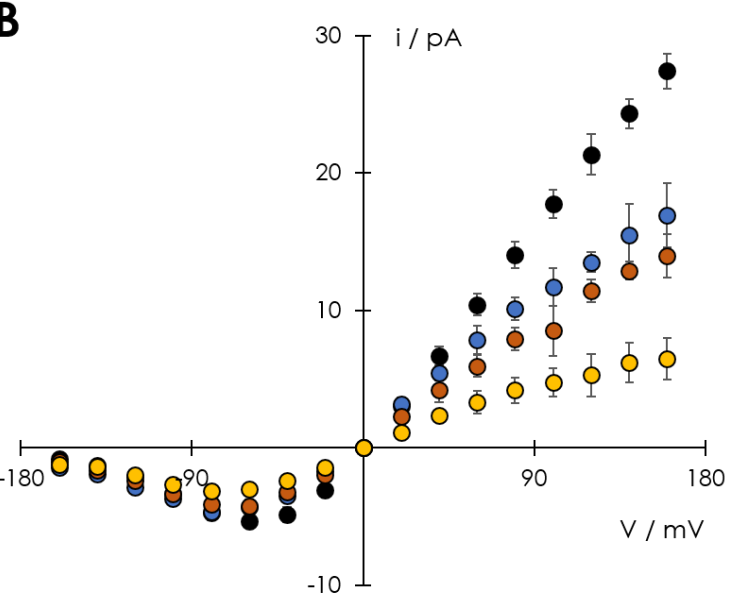

$-120 \mathrm{mV}$

Figure 21 - Comparison of KCVPBCV-1 and mutants L94H, L94K and L94R. (A) Exemplary gating events from single channel measurements of KCVPBCV-1, KCVPBCV-1 L94H, KCVPBCV-1 L94K and KCVPBCV-1 L94R at +120 $\mathrm{mV}$ (left) and -120 mV (right). c marks the closed state of the channels. Sections do not represent the overall open probability but should demonstrate the appearance of individual opening events in comparison to each other. For better visualization, data was subsequently filtered with $500 \mathrm{~Hz}$. (B) i/ $V$ curve and (C) open probability of KCVPBCV-1, KCVPBCV-1 L94H, KCVPBCV-1 L94K and KCVPBCV-1 L94R. Colors correspond to those from (A). Data are mean values \pm standard deviation for $n$ independent measurements (KCVPBCV-1: n=8; KCVPBCV-1 L94H: $n=3 ; K_{C V_{P B C V}-1}$ L94K: $n=3, K_{C V_{P B C V}-1}$ L94R: $n=3$ ). For better clarity only positive standard deviations are shown in (C). Measurements were performed under symmetrical conditions with $100 \mathrm{mM} \mathrm{KCl}$ and $10 \mathrm{mM}$ HEPES at $\mathrm{pH} 7$. 


\subsubsection{The effect of varying proton concentration on KCVPBCV-1 and KCVPBCV-1 $L 94 \mathrm{H}$}

All experiments that were performed so far were under the same experimental conditions: symmetrical measurements with $100 \mathrm{mM} \mathrm{KCl}, 10 \mathrm{mM}$ HEPES at pH7. Previous experiments have indicated that the introduction of an amino acid with a basic side chain at position 94 influences both, open probability and unitary single channel conductance of $\mathrm{KCV}_{\mathrm{PBCV}-1}$. While the single channel conductance is reduced to different degrees for all three mutants with basic side chains (KCVPBCV-1 L94H, KCVPBCV-1 L94K and KCVPBCV-1 L94R), the voltage dependency is reversed for only two (KCVPBCV-1 L94K and KCVPBCV-1 L94R). Additionally, the unitary single channel conductance of mutants with acidic side chains (KCVPBCV-1 L94D and KCVPBCV-1 L94E) is increased. Contrary to expectations, KCVPBCV-1 L94H showed no altered voltage dependency, despite its basic side chain. This is a first indication that the mechanism behind the change of properties for open probability and conductance are two separate ones. One assumption is that histidine at position 94 at $\mathrm{pH} 7$ is still in its deprotonated state. Experiments with varying proton concentrations should demonstrate, whether protonation of histidine leads to a similar effect on the open probability as KCVPBCV1 L94K and KCVPBCV-1 L94R. Experiments were performed under symmetrical conditions with $100 \mathrm{mM} \mathrm{KCl}$ and varying $\mathrm{pH}$ values. $10 \mathrm{mM}$ of a suitable buffer was added according to the corresponding $\mathrm{pH}$ value (pH 5: MES, pH 6: MES, pH 7: HEPES, pH 8: HEPES, pH 9: TAPS). For pH 4, the measuring solution was $90 \mathrm{mM}$ $\mathrm{KCl}$ and $10 \mathrm{mM} \mathrm{CH}_{3} \mathrm{COOK}$.

Fig. $22 \mathrm{~A}$ shows different exemplary sections of open events of KCVPBCV-1 $\mathrm{L94H}$ from pH 4 to $\mathrm{pH} 9$ at +120 $\mathrm{mV}$. For $\mathrm{pH} 4$ and $\mathrm{pH} 5$, the open events seem similar in their conductance and open dwell times. A change in gating is seen at $\mathrm{pH}$ 6. The black dotted box highlights a section of a measurement at $\mathrm{pH} 6$ with a higher resolution for better demonstration of the effect on channel fluctuations. Two different conducting states can be seen under this condition. While the lower conducting state is more defined $\left(\mathrm{O}_{1}\right)$, the second and higher conducting state is less stable and more difficult to define $\left(\mathrm{O}_{2}\right)$. With further increasing $\mathrm{pH}$ values and thus a decreasing $\mathrm{H}^{+}$concentration, open states with again a defined conductance level and shorter open dwell times appear. As already described in chapter 3.2.5 for $\mathrm{KCV}_{\mathrm{PBCV}-1} \mathrm{L94 \textrm {H }}$ at $\mathrm{pH} 7$, additional bursts appear irregularly with no defined conductance level or open probability. These bursts continue to appear at $\mathrm{pH} 8$ and $\mathrm{pH}$ 9. There is no consistency within these events, as shown for $\mathrm{pH} 8$; they can be higher or smaller, even within the same measurement. For $\mathrm{pH} 4$ and $\mathrm{pH} 5$, no such burst events are seen. While the conductance level is similar for $\mathrm{pH} 4$ and $\mathrm{pH} 5$, in the range of $\mathrm{pH} 5$ to $\mathrm{pH}$ 9, the conductance level of the stable open events steadily increases, until it seems to reach a plateau for high pH values. Fig. $22 \mathrm{~B}$ shows the corresponding i/ $\mathrm{V}$ curve for KCVPBCV-1 $\mathrm{L} 94 \mathrm{H}$ at different $\mathrm{pH}$ values (colored circles which correspond to fig. $22 \mathrm{~A}$ ), also compared to KCVPBCV-1 (black circles). Both values for $\mathrm{pH} 6$ are represented in different green tones. It confirms the assumptions which were based on inspection of the current traces. The conductance for KCVPBCV-1 L94H at pH 4, pH 5 and the lower conductance of $\mathrm{pH} 6$ are all in the same range. From pH 6 and higher, there is a steady rise of the unitary single channel conductance for KCVPBCV-1 $194 \mathrm{H}$, which is followed by a small increase in the $\mathrm{pH}$ between 8 to 9. The conductance of KCVPBCV-1 lies between the values for KCVPBCV-1 L94H at pH 8 and pH 9. 
$\mathrm{H}^{+}$concentration not only affects the conductance of KCVPBCV-1 L94H but also influences the open probability. Fig. $22 \mathrm{C}$ shows the different open probabilities of $\mathrm{KCV}_{\mathrm{PBCV}-1} \mathrm{~L}$ 94H from $\mathrm{pH} 4$ to $\mathrm{pH} 9$ (colored circles correspond to fig. 22 A), compared to KCVPBCV-1 (black circles). There is a clear shift in the voltage dependency with increasing $\mathrm{H}^{+}$concentrations. At $\mathrm{pH} 7, \mathrm{KCVPBCV}-1 \mathrm{L94H}$ was the only mutant with a basic side chain without a reversed voltage dependency (fig. 15 B). Due to the variability of pKa values of amino acids within a protein (Loewenthal et al., 1992; Takahashi et al., 1992; Edgcomb \& Murphy, 2002; Hansen \& Kay, 2014), it was assumed that histidine at $\mathrm{pH} 7$ might still be in its deprotonated state. If titration of histidine would be the explanation for a reversed voltage dependency, one would expect that with decreasing $\mathrm{pH}$ values an inversion of the voltage dependency would eventually occur for KCVPBCV-1 $L 94 \mathrm{H}$. Fig. $22 \mathrm{C}$ shows that this exactly is the case. With low pH values (pH 4 and 5), KCVPBCV-1 L94H exhibits the same voltage dependency as KCVPBCV-1 L94R and KCVPBCV-1 L94K (see chapter 3.2.5). With basic pH values the open probability is inverted compared to the wild type channel and increases with more positive voltages from values $\leq 1 \%$ to values around $10 \%$ at high positive voltages. While for $\mathrm{pH} 6$ a voltage dependency hardly exists, increasing the $\mathrm{pH}$ value further to 8 and 9 leads to an even more pronounced voltage dependency than for $\mathrm{KCV}_{\mathrm{PBCV}-1} \mathrm{L94H}$ and $\mathrm{KCV}_{\mathrm{PBCV}-1}$ at $\mathrm{pH}$ 7. These results strongly suggest that the inversion of the open probability is due to positive charges at the C-terminus of KCVPBCV-1. 
A
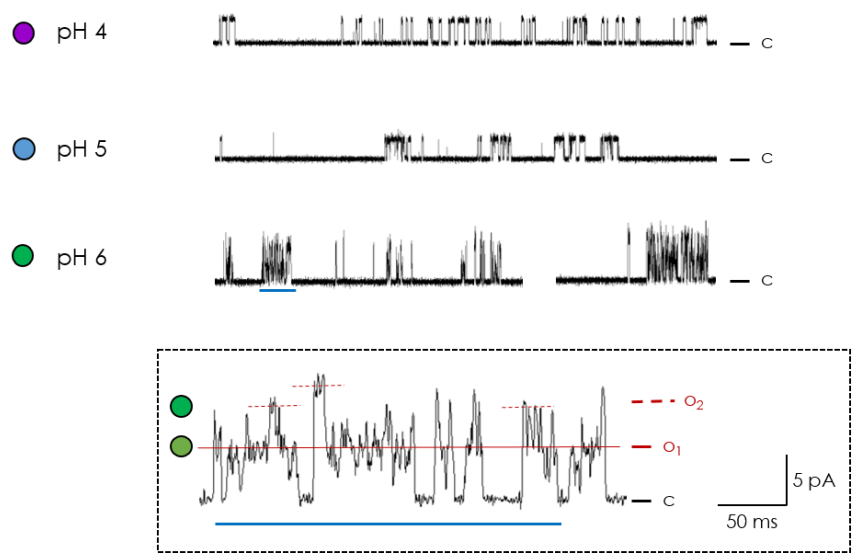

O $\mathrm{pH} 7$

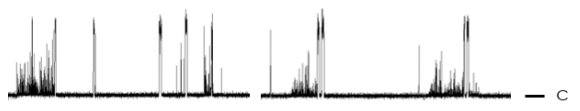

- $\mathrm{pH} 8$

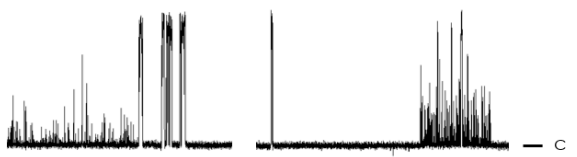

$\mathrm{pH} 9$

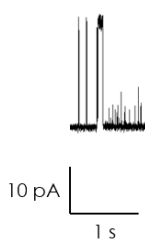

B

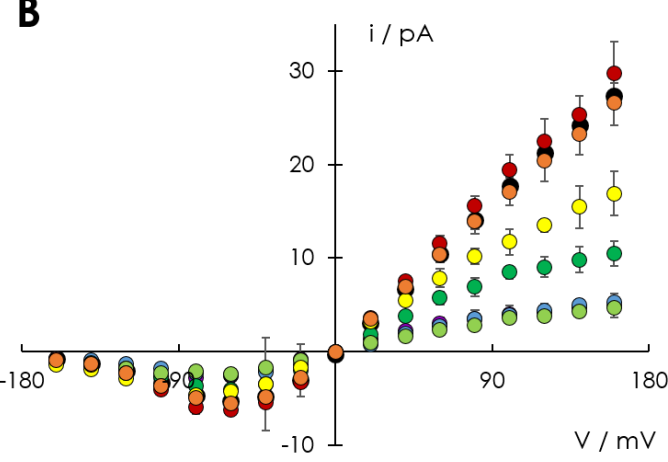

C

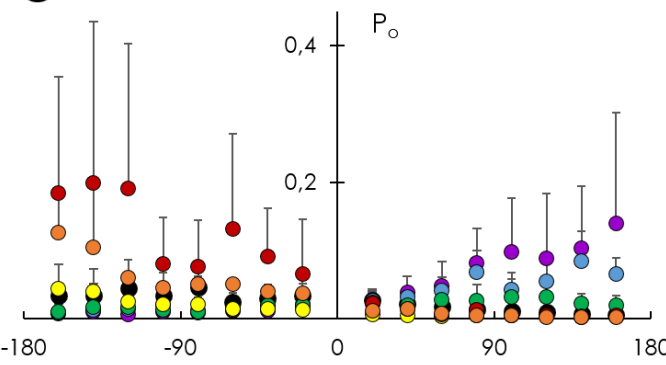

D $\quad v / m v$

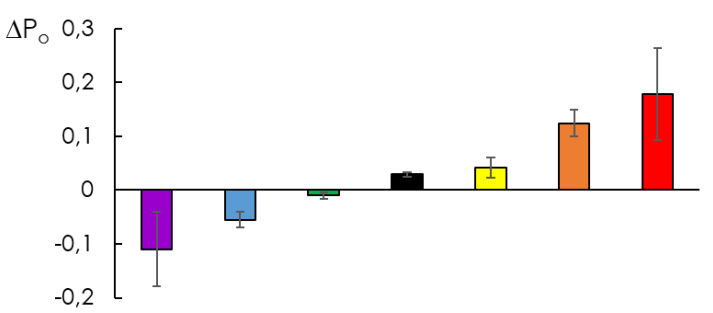

Figure 22 - Comparison of KCVPBCV-1 $\mathbf{L 9 4 H}$ at different $\mathrm{pH}$ values. (A) Exemplary gating events from single channel measurements of $\mathrm{KCV}_{\mathrm{PBCV}-1} \mathrm{L9} 9 \mathrm{H}$ at $+120 \mathrm{mV}$ at $\mathrm{pH} 4$ to $\mathrm{pH} 9$. A section of a measurement at $\mathrm{pH} 6$ is shown in the dotted box with a higher temporal resolution. $\mathrm{c}$ and o mark the closed and open state of the channels. Sections do not represent the overall open probability but should demonstrate the appearance of individual opening events in comparison to each other. For better visualization, data was subsequently filtered with $500 \mathrm{~Hz}$. (B) i/V curve and (C) open probability of KCVPBCV-1 L94H at different $\mathrm{pH}$ values. Data are mean values \pm standard deviation for $\mathrm{n}$ independent measurements. (D) Difference of the open probability between $-160 \mathrm{mV}$ and $+160 \mathrm{mV}$ for different $\mathrm{pH}$ values. Colors correspond to those from (A). For comparison, data of KCVPBCV-1 is shown in black. Data are mean values \pm standard errors for $n$ independent measurements $(\mathrm{pH} 4: \mathrm{n}=4, \mathrm{pH} 5: \mathrm{n}=3, \mathrm{pH}$ 6: $n=3, p H$ 7: $n=3, p H$ 8: $n=5, p H$ 9: $\left.n=4, K C V_{P B C V-1}: n=8\right)$. For better clarity only positive standard deviations are shown in (C). Measurements were performed under symmetrical conditions with 100 $\mathrm{mM} \mathrm{KCl}, 10 \mathrm{mM}$ buffer (pH 5,6: MES, $\mathrm{pH}$ 7,8: HEPES, $\mathrm{pH}$ 9: TAPS) and $90 \mathrm{mM} \mathrm{KCl}, 10 \mathrm{mM} \mathrm{CH}_{3} \mathrm{COOK}$ at $\mathrm{pH} 4$.

To further analyze the dependency of the unitary single channel conductance on $\mathrm{pH}$, the conductance of $\mathrm{KCV}_{\mathrm{PBCV}-1}$ was plotted as a function of the $\mathrm{H}^{+}$concentration (fig. 23). The data is shown as black filled circles. An exception is data obtained at $\mathrm{pH}$ 6. In this case I consider two conductance levels, a lower conductance level (empty black triangle) and a higher conductance (open red circle). The sigmoid curve progression shows that a plateau is reached at very high and very low $\mathrm{H}^{+}$concentrations $(G \min$ and 
Gmax, respectively). To determine the pKa value and the hill coefficient $h$, the data was fitted with equation (4):

$G=G_{\text {max }}-\left(G_{\text {max }}-G_{\text {min }}\right) \times \frac{\left[\mathrm{H}^{+}\right]^{\mathrm{h}}}{10^{-\mathrm{pK} a}+\left[\mathrm{H}^{+}\right]^{\mathrm{h}}}$

In a first attempt, the higher conductance for $\mathrm{pH} 6$ was considered. The resulting fit is shown in fig. 23 with a red line. The fit yields a hill-coefficient of 0.6 and a pKa value of 3.9. The pKa value of the side chain of histidine outside a protein is 6. Experiments have shown that the pKa value can change within the structure of a protein, depending on its environment to values between 6 and 7. (Loewenthal et al., 1992; Takahashi et al., 1992; Hansen \& Kay, 2014). Considering these values from the literature, a pKa value of 3.9 seems to be very low in relation to a histidine mediated effect. Due to the instability of the high conducting state of $\mathrm{KCVPBCV}_{-1} \mathrm{L94H}$ at $\mathrm{pH}$ 6, the fit was repeated without considering any value for $\mathrm{pH} 6$. An alternative fit is displayed as a green line, with a new hill coefficient of 0.8 and a pKa value of 5.5. Even though 5.5 is appreciably higher than 3.9, the new pKa value is still lower than expected from the literature. Due to the lack of data between $\mathrm{pH} 5$ and $\mathrm{pH}$ 7, the calculated value only indicates the $\mathrm{pH}$ range in which a change from protonated to deprotonated state is reached. Also, this value presumably does not resemble only the area around histidine 94 but the entire protein and its environment.

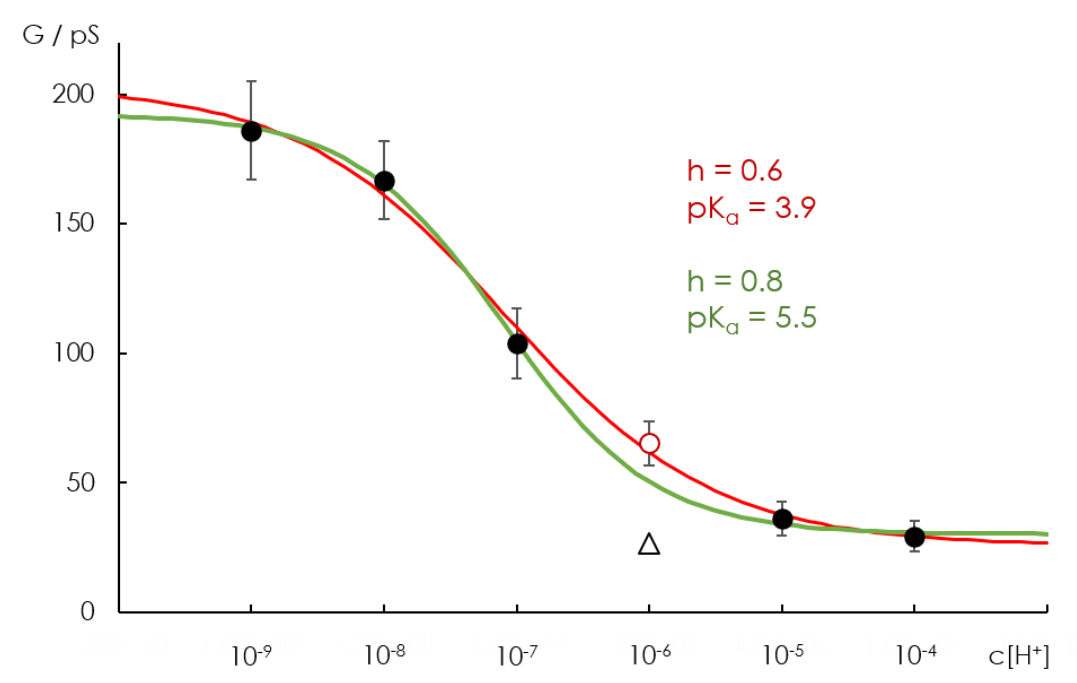

Figure 23 - Unitary single channel conductance of KCVPBCV-1 $\mathrm{L94H}$ as a function of $\mathrm{H}^{+}$concentration. Data points are mean values \pm standard deviation for $n$ independent measurements $(\mathrm{pH} \mathrm{4:} \mathrm{n}=4, \mathrm{pH}$ : $n=3, p H$ 6: $n=3, p H ~ 7: n=3, p H$ 8: $n=4, p H$ 9: $n=4$ ) for the unitary single channel conductance of KCVPBCV-1 L94H between $0 \mathrm{mV}$ and $+160 \mathrm{mV}$ at $\mathrm{pH} 4$ to $\mathrm{pH} 9$ as a function of $\mathrm{H}^{+}$concentration. The two values of $\mathrm{pH} 6$ are marked with an empty red circle and an empty black triangle. Data was fitted with equation (4). The red fit includes data point for $\mathrm{pH} 6$ of the higher conducting state (red empty circles), the green fit excludes both data points for $\mathrm{pH} 6$.

To validate if the mutated histidine at position 94 in $\mathrm{KCV}_{\mathrm{PBCV}-1} \mathrm{~L} 94 \mathrm{H}$ is protonated at $\mathrm{pH} 4$ and deprotonated at $\mathrm{pH}$ 9, additional experiments were performed where a $\mathrm{NiCl}_{2}$ solution was added to the cis chamber of the PLB setup. It is known that histidine coordinates bivalent metal ions, such as nickel, in its deprotonated state (Krämer et al., 1996; Perrin \& Sharma, 1967). If histidine is freely accessible from the intracellular side 
(cis chamber), it should be protonated at high $\mathrm{H}^{+}(\mathrm{pH} 4)$ and deprotonated at low $\mathrm{H}^{+}(\mathrm{pH}$ 9). Since this position is near the entrance of the cavity for $\mathrm{K}^{+}$ions, the coordination of $\mathrm{Ni}^{2+}$ through deprotonated histidine might lead to differences in the single channel gating properties of KCVPBCV-1 L94H. These differences are only expected for measurements of KCVPBCV-1 L94H at pH 9. For this experiment, KCVPBCV-1 L94H was first measured under symmetrical conditions with $100 \mathrm{mM} \mathrm{KCl}$ at pH 4 and pH 9. Subsequently, $10 \mu \mathrm{M} \mathrm{NiCl}_{2}$ solution was added to the cis chamber and carefully resuspended. Fig. 24 shows exemplary amplitude histograms at $+120 \mathrm{mV}$ of $\mathrm{KCV}_{\mathrm{PBCV}-1} \mathrm{~L} 94 \mathrm{H}$ at $\mathrm{pH} 9$ before and after addition of $10 \mu \mathrm{M} \mathrm{NiCl}_{2}$. Before addition (fig. 24, left side), two distinct levels, representing the closed (c) and open (o) state, can be identified. After addition of $10 \mu \mathrm{M} \mathrm{NiCl}_{2}$, a change in gating is observable. Both, single channel traces and amplitude histogram now show not one, but three different, distinct levels representing varying conductive states of the channel. Repetition of this experiment with KCVPBCV-1 L94H at pH 4 shows no such effect after addition of $\mathrm{NiCl}_{2}$ (fig. 25).
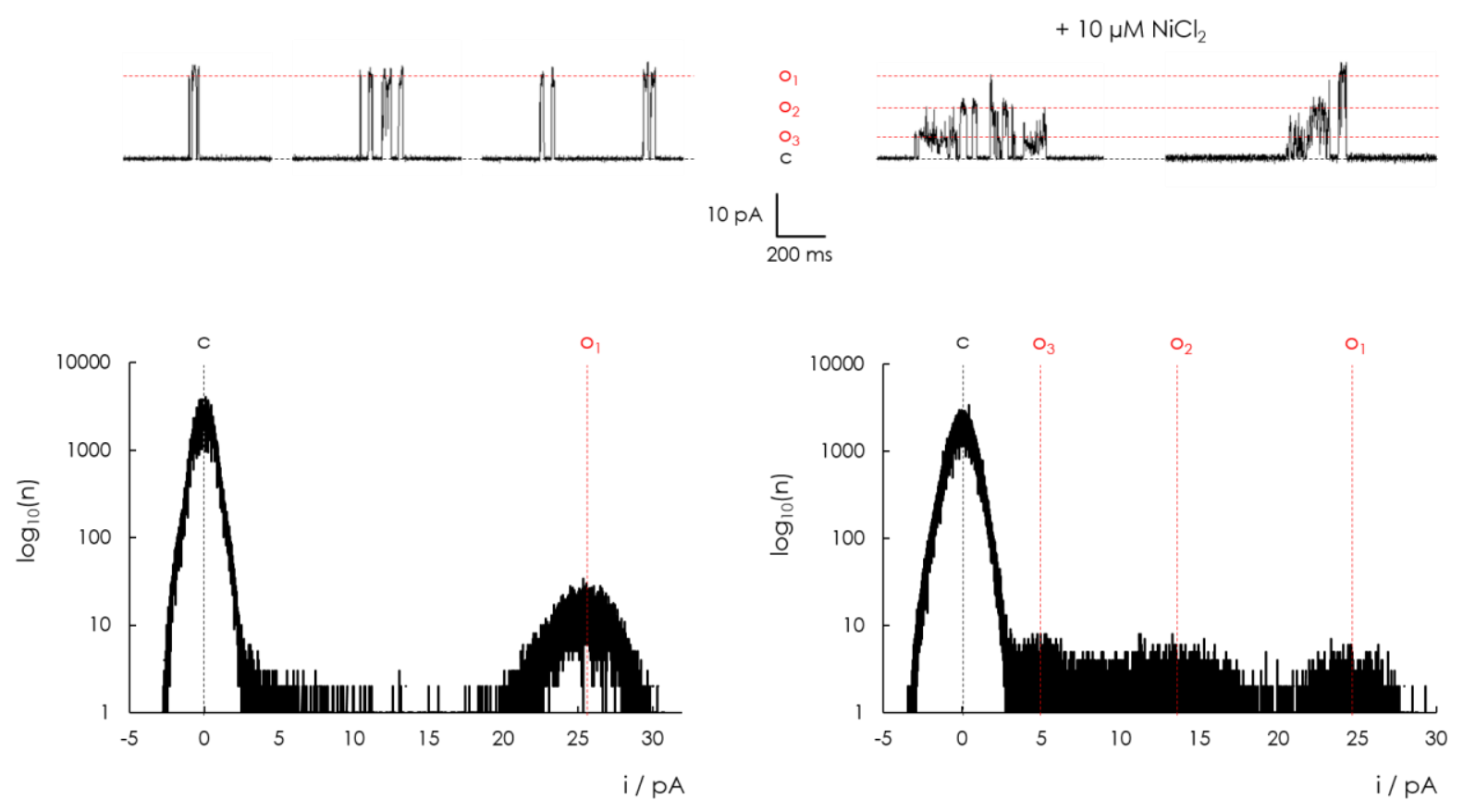

Figure 24 - Effect of $\mathrm{Ni}^{2+}$ on KCVPBCV-1 $\mathbf{~} 94 \mathrm{H}$ at pH 9. Excerpts of open time events (top) of KCVPBCV-1 L94H at $\mathrm{pH} 9$ at $+120 \mathrm{mV}$ before (left) and after (right) addition of $10 \mu \mathrm{M} \mathrm{NiCl} 2$ to the cis compartment. For better visualization, data was subsequently filtered with $500 \mathrm{~Hz}$. Corresponding amplitude histograms to a 5-minute measurement (bottom) KCVPBCV-1 $\mathrm{L94H}$ at $+120 \mathrm{mV}$ before (left) and after (right) $\mathrm{NiCl}_{2}$ addition. The black dotted line marks the closed (c) level, while the red dotted lines mark the different open (o) levels. Measurements were performed under symmetrical conditions with $100 \mathrm{mM} \mathrm{KCl}$ and $10 \mathrm{mM}$ TAPS at $\mathrm{pH} 9$. 

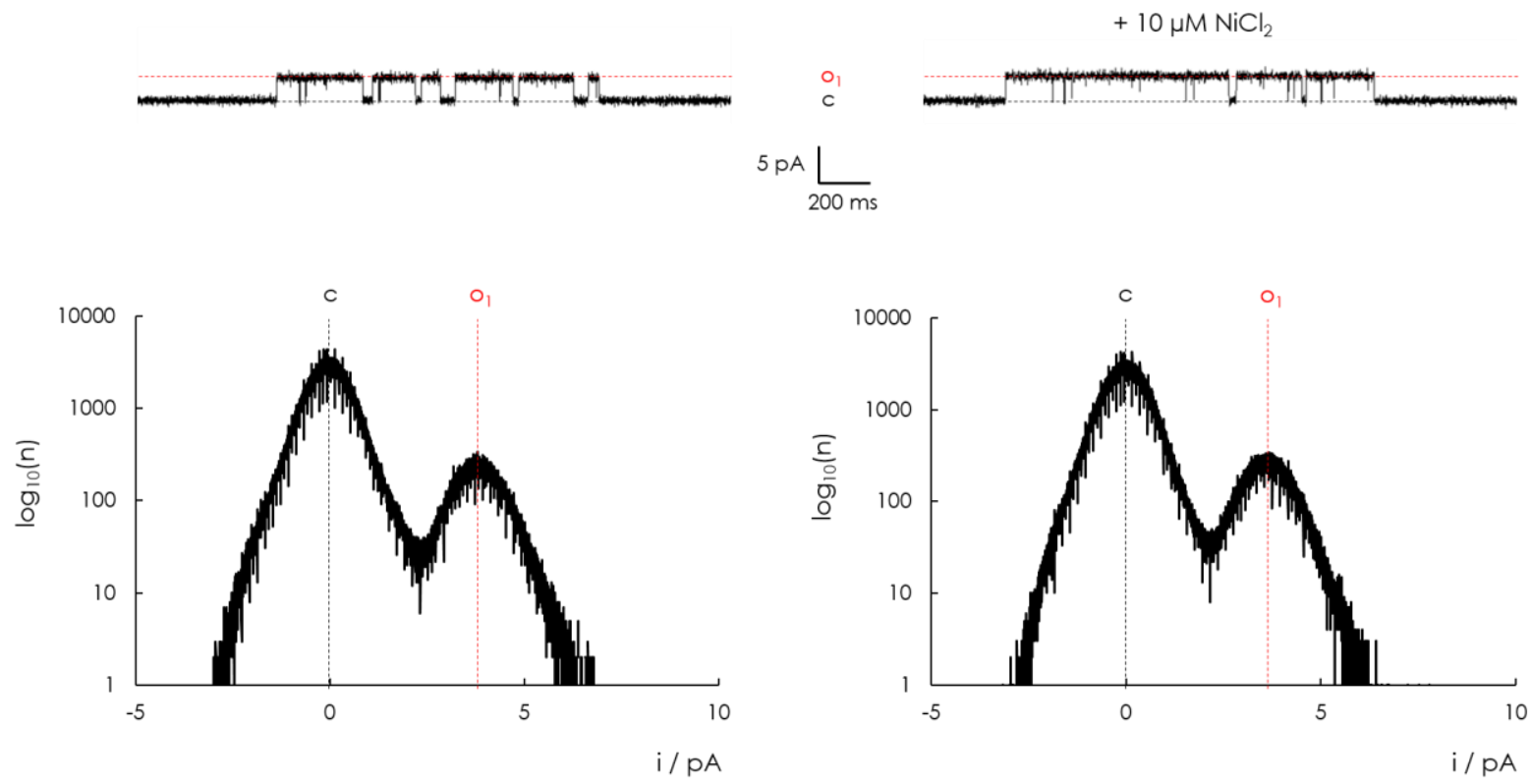

Figure 25 - Effect of $\mathrm{Ni}^{2+}$ on $\mathrm{KCV}_{\mathrm{PBCV}-1} \mathrm{L94H}$ at pH 4. Excerpts of open time events (top) of KCVPBCV-1 L94H at $\mathrm{pH} 4$ at $+120 \mathrm{mV}$ before (left) and after (right) addition of $10 \mu \mathrm{M} \mathrm{NiCl} 2$ to the cis compartment of the bilayer setup. For better visualization, data was subsequently filtered with $500 \mathrm{~Hz}$. Corresponding amplitude histograms to a 5-minute measurement (bottom) KCVPBCV-1 L94H at $+120 \mathrm{mV}$ before (left) and after (right) $\mathrm{NiCl}_{2}$ addition. The black dotted line marks the closed (c) level, while the red dotted line marks the open (o) level. Measurements were performed under symmetrical conditions with 90 $\mathrm{mM} \mathrm{KCl}$ and $10 \mathrm{mM} \mathrm{CH}_{3} \mathrm{COOK}$ at $\mathrm{pH} 9$.

The results of these experiments indicate that deprotonated histidine at position 94 is capable of coordinating nickel. The histidine-nickel complex might hinder the flow of potassium ions through the pore. To rule out the possibility of such an effect already occurring in the wild type channel, the same experiments were performed with $\mathrm{KCV}_{\mathrm{PBCV}-1} .10 \mu \mathrm{M} \mathrm{NiCl}_{2}$ was added to the cis compartment at $\mathrm{pH} 9$ and $\mathrm{pH} 4$ in the same way as for KCVPBCV-1 L94H. In both cases, no such effect on channel gating could be observed (data not shown). This proves that the effect is solely due to the protonation state of histidine in position 94.

To verify if the effect of different proton concentrations is due to the mutation L94H in KCVPBCV-1, the wild type channel must also be examined at different $\mathrm{pH}$. The experiments performed for KCV $\mathrm{PBCV}_{-1} \mathrm{~L}$ 94H were therefore repeated for $\mathrm{KCVPBCV}_{\mathrm{P}-1}$. PLB measurements were performed in the range from $\mathrm{pH} 4$ to 9 with buffer solutions containing $100 \mathrm{mM} \mathrm{KCl}$. Corresponding single channel traces of $\mathrm{KCV}_{\mathrm{PBCV}-1}$ from $\mathrm{pH} 4$ to $\mathrm{pH}$ 9 are shown in fig. $26 \mathrm{~A}$. With decreasing $\mathrm{H}^{+}$concentrations, a continuous increase of the current amplitude can be seen. Dwell times of open events are very short, although for $\mathrm{pH} 4$ they seem to be slightly longer. For pH 8 two gating types could be observed. Type 1 (orange triangles) has a much higher open probability at positive voltages than type 2 (orange circles). This is shown in more detail in fig. 27: five independent measurements were made of $\mathrm{KCVPBCV}_{-1}$ at $\mathrm{pH}$ 8. Individually plotted, two populations can be identified with opposite voltage dependencies. Two measurements can be assigned to type 1 (grey triangles). Here the open probability increases from $\sim 5 \%$ at negative to $\sim 20 \%$ at positive voltages. The open probability for type 2 (grey circles) decreases from $\sim 5 \%$ to $\leq 1 \%$ with more positive membrane 
potentials. Three measurements could be assigned to type 2. The unitary single channel conductance for both gating types is the same.

Fig. $26 \mathrm{~B}$ shows the corresponding i/V relation of $\mathrm{KCVPBCV}_{-1}$ from $\mathrm{pH} 4$ to 9, the colors correspond to those from fig. 26 A. The steady increase of unitary single channel conductance that was indicated from observation of the single channel traces is confirmed here. There is a steady increase of the conductance from $\mathrm{pH} 4$ to $\mathrm{pH} 8$. Between $\mathrm{pH} 8$ and $\mathrm{pH} 9$ there is no further increase. Compared to KCVPBCV-1 L94H there is no steady shift seen in the voltage dependency of the open probability for KCVPBCV-1 at different pH (fig. $26 \mathrm{C}$ ). The open probabilities are in the same range with the same voltage dependency, with two exceptions: KCVPBCV-1 at $\mathrm{pH} 4$ and gating type 2 of KCVPBCV-1 at $\mathrm{pH}$ 8. Both have an inversed voltage dependency, increasing up to $\sim 20 \%$ with positive voltages. For all other $\mathrm{pH}$, the open probability of $\mathrm{KCV}_{\mathrm{PBCV}-1}$ decreases from $\sim 5 \%$ at negative to $\leq 1 \%$ at positive voltages.

A

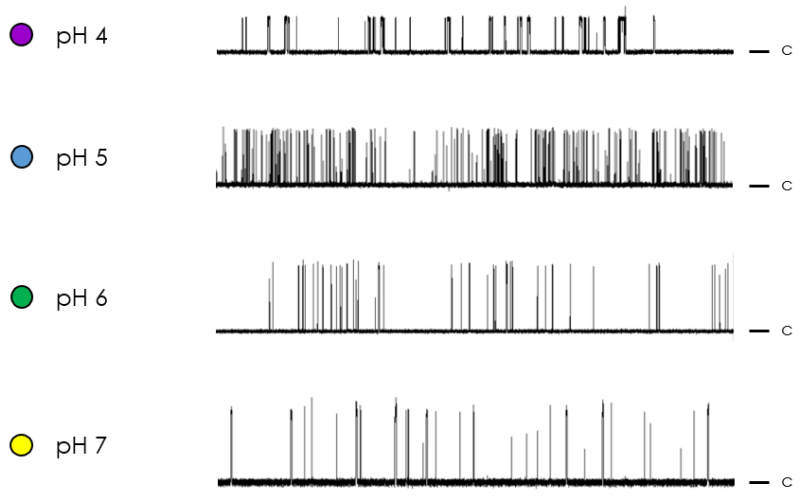

$\triangle \mathrm{pH} 8$

$\mathrm{pH} 8$

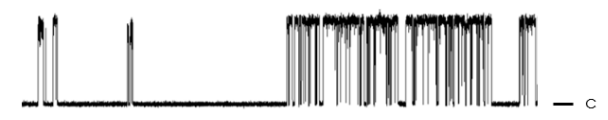

$\mathrm{pH} 9$

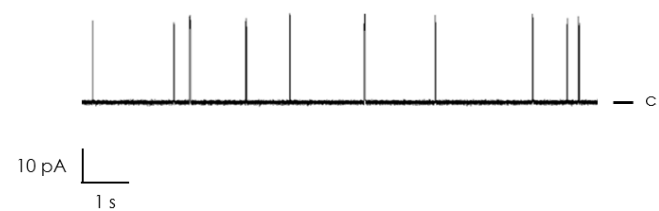

B

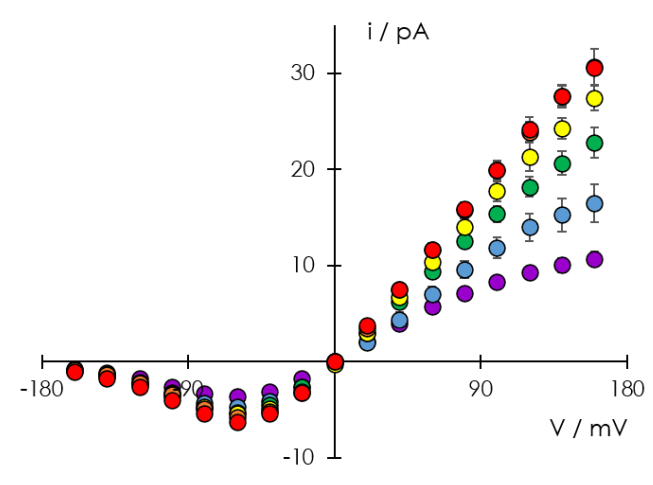

C

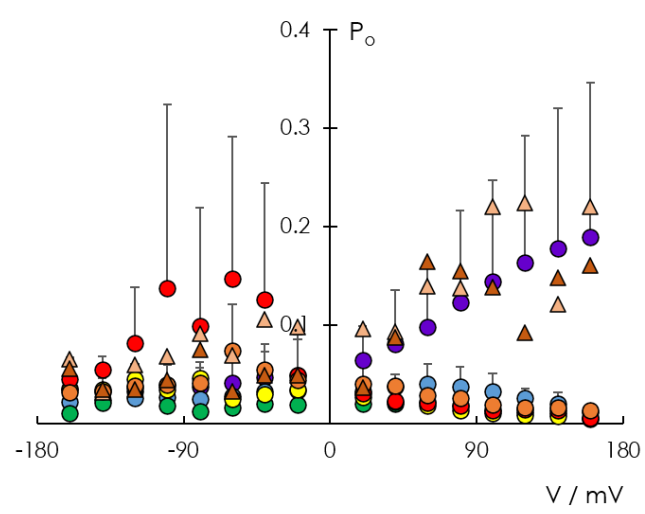

Figure 26 - Comparison of $\mathrm{KCVPBCV}_{\mathrm{P}}$ at different $\mathrm{pH}$ values. (A) Exemplary gating events from single channel measurements of $\mathrm{KCVPBCV}_{-1}$ at $+120 \mathrm{mV}$ at $\mathrm{pH} 4$ to $\mathrm{pH}$ 9. For $\mathrm{pH} 8$ two different sections are shown due to two different gating modes (orange circles and triangles). c marks the closed state of the channels. Sections do not represent the overall open probability but should demonstrate the appearance of individual opening events in comparison to each other. For better visualization, data was subsequently filtered with $500 \mathrm{~Hz}$. (B) i/V curve and (C) open probability of KCVPBCV-1 at different $\mathrm{pH}$ values. Colors correspond to those from (A). Data are mean values \pm standard deviation for $n$ independent measurements $(\mathrm{pH}$ 4: $\mathrm{n}=6, \mathrm{pH}$ 5: $\mathrm{n}=6, \mathrm{pH}$ 6: $\mathrm{n}=5, \mathrm{pH} 7: \mathrm{n}=8, \mathrm{pH}$ 8: $\mathrm{n}=3$ (circles), $\mathrm{pH}$ 9: $\mathrm{n}=3$ ). Data of $\mathrm{pH} 8$ (triangles) are two independent measurements. For better clarity only positive standard deviations are shown in (C). Measurements were performed under symmetrical conditions with $100 \mathrm{mM} \mathrm{KCl}, 10 \mathrm{mM}$ buffer (pH 5,6: MES, pH 7,8: HEPES, pH 9: TAPS) and $90 \mathrm{mM} \mathrm{KCl}, 10 \mathrm{mM} \mathrm{CH}_{3} \mathrm{COOK}$ at $\mathrm{pH} 4$. 


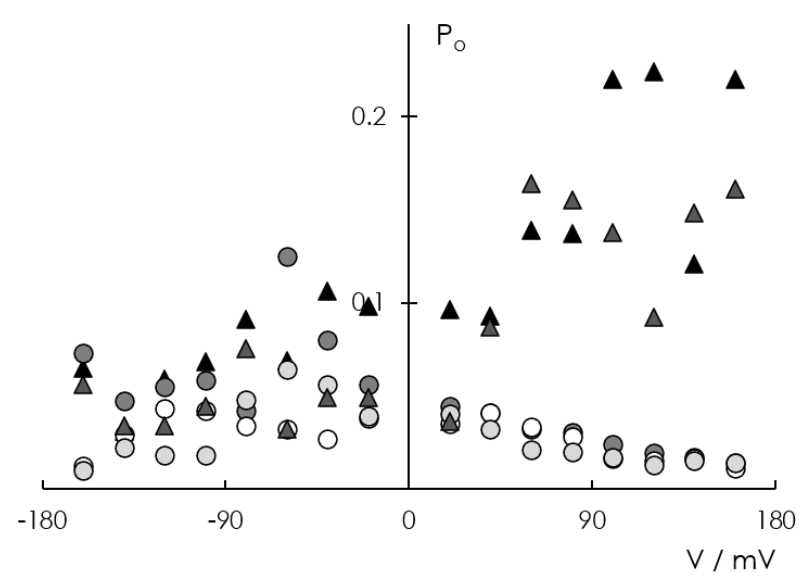

Figure 27 - Two different gating types of $\mathrm{KCV}_{\mathrm{PBCV}-1}$ at $\mathrm{pH}$ 8. Data points from 5 individual measurements of $\mathrm{KCV}_{\mathrm{PBCV}-1}$ at $\mathrm{pH}$ 8. Two different gating types can be seen, type 1 is marked with grey triangles $(n=2)$ and type 2 with grey circles $(n=3)$. Measurements were performed under symmetrical conditions with $100 \mathrm{mM} \mathrm{KCl}$ and $10 \mathrm{mM}$ HEPES at $\mathrm{pH} 8$.

The results show that already the wild type channel $\mathrm{KCVPBCV}_{-1}$ is $\mathrm{pH}$ sensitive. To compare the proton dependent alteration of the unitary single channel conductance of KCVPBCV-1 and KCVPBCV-1 L94H, the conductance of $\mathrm{KCV}_{\mathrm{PBCV}-1}$ was plotted as a function of $\mathrm{H}^{+}$(fig. $28 \mathrm{~A}$, black circles) together with $\mathrm{KCV}_{\mathrm{PBCV}-1}$ L94H (fig. 28 A, empty circles). As described previously, considering the higher conductance for KCVPBCV-1 $\mathrm{L} 94 \mathrm{H}$ at $\mathrm{pH} 6$, the increase of unitary single channel conductance is titratable and could be fitted with equation (4). For KCVPBCV-1 the curve progression is also slightly sigmoidal, although with a lower steepness and cooperativity compared to $\mathrm{KCVPBCV}_{-1} \mathrm{L94H}$. The plateau for $\mathrm{KCVPBCV}_{-1}$ at low $\mathrm{H}^{+}$concentrations is clear, while at high $\mathrm{H}^{+}$concentrations it seems to lie outside the measured $\mathrm{pH}$ range. Fig. 28 shows both fits as dotted lines. The different progression of the two curves and their corresponding fits imply that mutation from leucine at position 94 to histidine alters the wild type channels sensitivity towards $\mathrm{H}^{+}$concentration. 


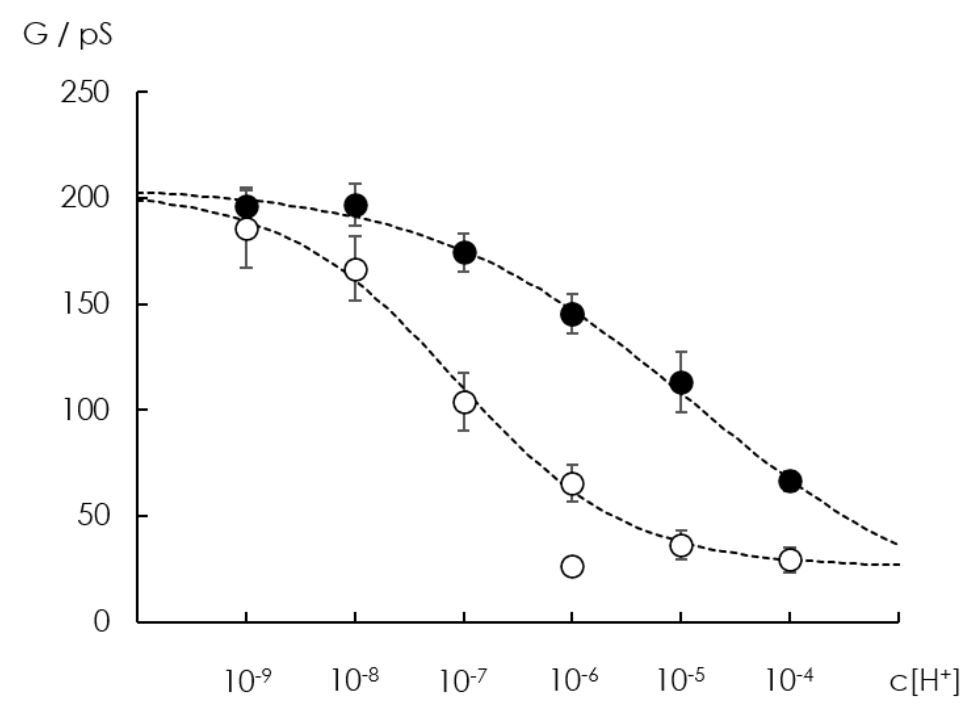

Figure 28 - Unitary single channel conductance of KCVPBCV-1 and KCVPBCV-1 $\mathbf{L}$ (94H as a function of $\mathrm{H}^{+}$ concentration. (A) Unitary single channel conductance of KCVPBCV-1 and KCVPBCV-1 L94H as a function of $\mathrm{H}^{+}$concentration. Data points are mean values \pm standard deviation for $\mathrm{n}$ independent measurements (KCVPBCV-1: $\mathrm{pH} 4: \mathrm{n}=6, \mathrm{pH}$ 5: $\mathrm{n}=6, \mathrm{pH}$ 6: $\mathrm{n}=5, \mathrm{pH}$ 7: $\mathrm{n}=8, \mathrm{pH}$ 8: $\mathrm{n}=5, \mathrm{pH}$ 9: $\mathrm{n}=3 ; \mathrm{KCV} \mathrm{VBCV}_{-1}$ L94H: $\mathrm{pH}$ 4: $\mathrm{n}=4, \mathrm{pH}$ 5: $\mathrm{n}=3, \mathrm{pH}$ 6: $\mathrm{n}=3, \mathrm{pH}$ 7: $\mathrm{n}=3, \mathrm{pH}$ 8: $\mathrm{n}=4, \mathrm{pH}$ 9: $\mathrm{n}=4$ ). Data of KCVPBCV-1 and KCVPBCV$1 \mathrm{L94H}$ were fitted with equation (4). Both fits are shown as black dashed lines.

\subsubsection{Discussion}

$\mathrm{KCVPBCV}_{-1}$ is one of the smallest known, functional $\mathrm{K}^{+}$channels. Each subunit consists of only 94 aa, merely containing two transmembrane helices, connected by a 44 aa sequence containing the pore loop with the SF, a short 12 aa cytosolic N-terminus and no free C-terminus (Doyle et al., 1998). This basic layout resembles the pore module of bigger and more complex channels. Its small size makes it an ideal model system to understand structure/function relations in this channel with the possibility of extrapolating these findings to complex $\mathrm{K}^{+}$channels with the same pore architecture.

In the work of Gebhardt (2010), the naturally occurring leucine at position 94 of KCVPBCV-1 was considered an important position within the protein and therefor scrutinized. On the one hand, the free charge of the carboxyl group at the end of the sequence is essential for channel gating, due to the formation of salt bridges with the positively charged amino acids within the N-terminus of the channel (Hertel et al., 2010; Tayefeh et al., 2009). On the other hand, it was also revealed that the exchange of the side chain at position 94 influences channel activity. For these experiments, computational data was compared to experimental data from yeast complementation assays. Since the latter method is rather indirect and does not provide information about changes on the single channel level, for this study KCVPBCV-1 L94X mutants ( $X$ standing for mutation to all other proteinogenic amino acids of the standard genetic code) were examined with the PLB method. Single channel analyses enable the detection of changes in the unitary single channel conductance and open probability of each individual mutant. The experiments should also give an impression of the extent to which the results of these two methods are comparable. 
In the first part of the experiment, KCVPBCV-1 and KCVPBCV-1 L94X were examined for differences in unitary single channel conductance and open probability. For the majority of mutations, neither a significant difference in conduction nor open probability was seen. One aim of this study was to compare the results of yeast complementation assays with data from PLB measurements. Yeast complementation assays compare the growth of potassium uptake deficient (trk $1 \Delta /$ trk2 $\Delta$ ) yeasts expressing heterologous ion channels versus growth of trk $1 \Delta /$ trk2 $\Delta$ yeasts without expression of heterologous ion channels on media with a low potassium concentration (Bagriantsev \& Minor, 2013). Fig. 29 shows results of yeast complementation assays of strain SGY1528 from M. Gebhardt (TU Darmstadt, unpublished data) for all 20 channels, KCVPBCV-1 and the nineteen mutants KCVPBCV-1 L94X. The bars on the right show yeast complementation with $\mathrm{KCVPBCV}_{\mathrm{P}}$ and $\mathrm{KCV}_{\mathrm{PBCV}-1} \mathrm{~L} 94 \mathrm{X}$ after $\mathrm{Oh}, 24 \mathrm{~h}$ and $48 \mathrm{~h}$ in liquid media with $0.5 \mathrm{mM} \mathrm{KCl}$. The cells transformed with an empty vector pYES2 cannot grow in media with a low potassium concentration since it lacks an endogenous potassium uptake system and therefor serves as a negative control. No growth can be seen, even after 48h. In all transformed cells expressing KCVPBCV-1 or KCVPBCV-1 L94X (expect of D and N), yeast growth could be observed in liquid media, which is increased after $48 \mathrm{~h}$ compared to $24 \mathrm{~h}$, indicating that the channel and nearly all channel mutants are in principle functional. Compared to the wild type channel (framed by a red box), some mutants show a similar, or only slightly more or less growth $(A, I, P, Q, T, V, W)$, while some show a reduction $(C, D, E, G, N, R, S)$ or an increase $(F$, $H, K, M, Y)$ of growth. However, on plate only the mutants $F, H$ T and $Y$ complement the trk $1 \Delta /$ trk2 $\Delta$ growth deficiency of the yeasts in the same way as the wild type channel, while A, C, D, E, G, I, N, R, S and W do not complement the trkl $\Delta /$ trk2 $\Delta$ growth deficiency. Mutants $K, M, P, Q$ and $T$ show a partial complementation of the trk $1 \Delta /$ trk2 $\Delta$ growth deficiency. After extensive comparison of the results from the yeast complementation assay (fig. 29) and the results from KCVPBCV-1 and KCVPBCV-1 L94X measured on a single channel level with the PLB method (fig. 15, fig. 16 \& fig. 17), no commonalities could be found. A reduced growth could be seen for KCVPBCV-1 L94D and KCVPBCV-1 L94E. In PLB experiments, these two mutants showed a slight increase of unitary single channel conductance. A higher conductance might be toxic for the cells, due to a possible collapse of the membrane potential. L94P on the other hand has no higher unitary single channel conductance but a higher mean open probability, which also means an increased potassium uptake. Growth for KCVPBCV-1 L94P is however nearly in the same range as the wild type channel, or even slightly increased. Also, KCVPBCV-1 L94F and KCVPBCV-1 L94N do not differ greatly in their unitary single channel conductance or mean open probability in PLBs, yet in yeast KCVPBCV-1 L94F leads to a great increase of yeast growth while KCVPBCV-1 L94N has the complete opposite effect. Further comparison leads to no solid similarities between the two methods. While yeast complementation assays are a great screening method for testing the functionality of channels, it provides insufficient information for a detailed characterization of the individual mutants on the single channel level. Changes in yeast growth could also be due to factors such as the amount of protein expression or protein sorting which cannot be differentiated within the results. 


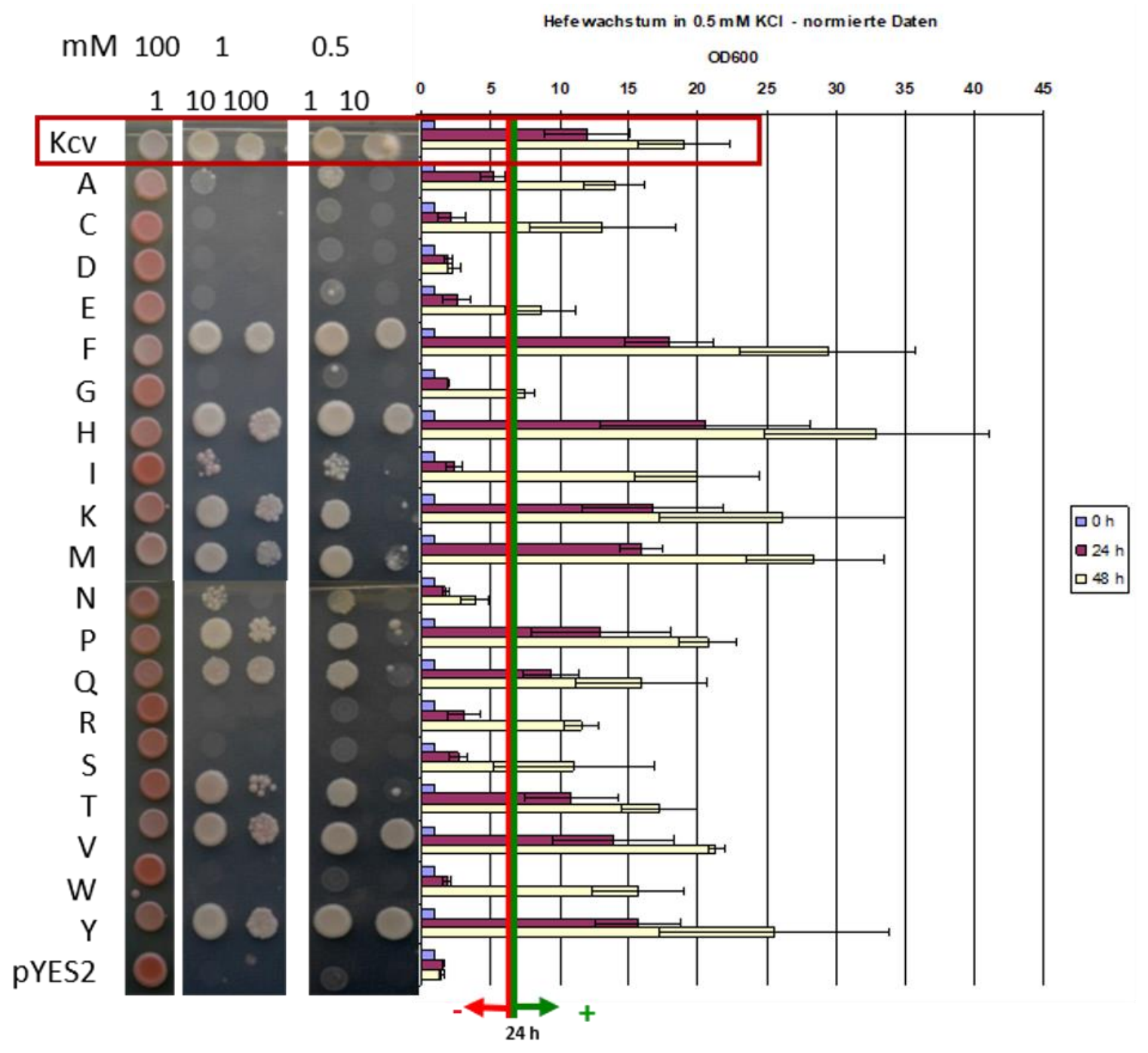

Figure 29 - Yeast complementation assays of the strain SGY1528 transformed with PYES2, KCVPBCV-1 and KCVPBCV-1 L94X. Yeast growth assays of KCVPBCV-1 and the mutants KCVPBCV-1 L94X, where X represents the amino acid of the corresponding mutation, abbreviated with the one letter code. The assays on the left were done on plates with three different potassium: $100 \mathrm{mM} \mathrm{KCl}$ as control and $1 \mathrm{mM}$ as well as $0.5 \mathrm{mM} \mathrm{KCl}$ as selective conditions. $\mathrm{KCVPBCV-1}$, KCVPBCV-1 L94X and an empty vector as negative control (pYES2), were spotted in different dilutions of an OD600 solution: 1 (undiluted), 1:10 and 1:100. Yeast growth in liquid media with $0.5 \mathrm{mM} \mathrm{KCl}$ is additionally shown as bars on the right side, measured by the corresponding OD600 after Oh (light purple), 24h (pink) and 48h (yellow). KCVPBCv1 is framed by a red box. Unpublished data from M. Gebhardt, TU Darmstadt.

Mutations KCVPBCV-1 L94P and KCVPBCV-1 L94C were the only two mutations causing a clear increase of the open probability of $\mathrm{KCV}_{\mathrm{PBCV}-1}$, accompanied by a more pronounced voltage dependency. For KCVPBCV-1 L94P, an additional effect was seen. The unitary single channel conductance was noticeably reduced compared to the KCVPBCV-1 (fig. 18). However, for short dwell times the channel seems to switch into a state with a higher conductance, which lies in the same range as for the wild type. Proline is known to appear in two conformations: cis and trans. The greatly favored conformation of amino acids after integration into the peptide chain is the trans configuration (Zimmerman \& Scheraga, 1976). Proline is an exception, it can be found in both, cis and trans configuration (Wedemeyer et al., 2002; Zimmerman \& Scheraga, 1976). Studies have shown that cis-trans-isomerization can take place within the protein (Steinberg et al., 
1960), which can alter channel properties (Sarkar et al., 2007). Switching between cis and trans conformation of proline is even known to be part of the gating mechanism of an ion channel (Lummis et al., 2005). It is possible that proline persists most of the time in one conformation, which is energetically more stable but hinders ion flow compared to the wild type, causing a reduction of the unitary single channel conductance. If for short occasions the energy barrier is reduced, a transient switching from one state to the other and a resulting conformational change might be a possible explanation for these shortlasting states of higher conductance.

Mutation of leucine 94 to cysteine has no notable effect on the unitary single channel conductance of KCVPBCV-1 but causes a great increase of mean open probability and voltage dependency (fig. $19 \mathrm{~B} \& \mathrm{C}$ ). There was also a great variety of gating types seen for KCVPBCV-1 L94C, which cannot be categorized into only two distinct modes (fig. 20). An obvious assumption was the formation of disulfate bonds between the newly introduced cysteine and another cysteine within the protein, leading to a stabilization of the open state. This assumption could not be confirmed, for addition of DTT to the measurement did not reduce the open probability (fig. $19 \mathrm{D \& E}$ ). DTT in the concentration of $5 \mathrm{mM}$ is known to be sufficient for the reduction of possible disulfide bonds (Rauh et al., 2017).

It remains inconclusive what causes this increase in the open probability of KCV $\mathrm{PBCV}_{-1}$ L94P and KCV $\mathrm{PBCV}_{\mathrm{P}-1}$ L94C. Different parameters, such as side chain properties, hydrophobicity and molecular weight could be ruled out, as well as formation of disulfate bridges in KCVPBCV-1 L94C. The C-terminal end of the channel is known to be involved in channel gating, due to the formation of salt bridges between the free charge of the carboxyl group of the last amino acid and the positively charged amino acids of the N-terminus. While mutation of the last amino acid changes the side chain properties in this position, the free negative charge remains, so salt bridge formation should not be prevented. During a stable formation of these salt bridges, $\mathrm{K}^{+}$ions can freely enter and exit the cavity. By disruption, $\mathrm{K}^{+}$ions bind to the four free negative charges of the carboxyl groups at the end of the individual subunits, blocking the entrance and exit for further $\mathrm{K}^{+}$ions. The low open probability of $\mathrm{KCV}_{\mathrm{PBCV}-1}$ indicates that the formation of these salt bridges is rare and short lasting. An increase in the open probability could be due to the stabilization of the salt bridges. It could be possible that mutation of leucine 94 to cysteine and proline causes a conformational rearrangement at the $\mathrm{C}$-terminal end of the channel, bringing the free negative charge of the carboxyl group into closer proximity to the $\mathrm{N}$-terminus, which leads to an increased stability of the salt bridge, especially at negative membrane potentials. While this last hypothesis sounds plausible, it does not explain why exactly these two amino acids would cause such an effect, while other amino acids with similar properties do not.

Besides the great difference in open probabilities for KCVPBCV-1 L94P and KCVPBCV-1 L94C, another noticeable feature could be observed. Mutants in which leucine 94 was exchanged for amino acids with basic side chains (L94H, L94K, L94R) seem to have two effects on gating of KCVPBCV-1. Firstly, all three mutants lower the unitary single channel conductance of KCVPBCV-1 to a varying degree (fig. $21 \mathrm{~B}$ ). It is also interesting to see that mutants with acidic side chains (L94D and L94E) show a slight increase in conductance. Taken together the data suggest that cationic amino acids reduce, while anionic amino 
acids favor the unitary conductance of KCVPBCV-1. Secondly, even though the mean open probability is similar to $\mathrm{KCV}_{\mathrm{PBCV}-1}$, for two of the three mutants (L94K and L94R) a reversed voltage dependency was observed (fig. 21 C). Further experiments showed that this reversed voltage dependency develops also

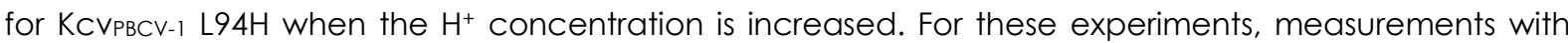
$\mathrm{KCV}_{\mathrm{PBCV} \text {-1 }} \mathrm{L94H}$ were performed with varying $\mathrm{H}^{+}$concentrations from $\mathrm{pH} 4$ to $\mathrm{pH}$ 9, to investigate the titratability of the histidine residue at position 94.

From the single channel traces (fig. 22 A) and the resulting pKa value (5.5) from the fit in fig. 23 it is clear that at $\mathrm{H}^{+}$concentrations of around $\mathrm{pH} 6$ KCVPBCV-1 $\mathrm{L} 94 \mathrm{H}$ undergoes some sort of conformational change which in turn leads to an altered gating. At $\mathrm{pH} 4$ and $\mathrm{pH} 5$ the channel maintains a state with an identical, but reduced conductance. At $\mathrm{pH} 6$ this gating type switches between the latter and a second, less stable state with a higher conductance. This can also be confirmed by the calculated pKa value of 5.5 (fig. 23). With decreasing $\mathrm{H}^{+}$concentrations the higher conducting state is stabilized, with occasional bursts with an undefined conductance. Experiments with KCVPBCV-1 at different $\mathrm{pH}$ values have shown that the wild type channel is already $\mathrm{pH}$ sensitive. However, the dose/response curve for the of single channel conductance as a function of pH (fig. 28) demonstrates that the effect is different between the wild type channel and the KCVPBCV-1 L94H mutant.

Noticeably, it appears that the curves in fig. 28 for both, KCVPBCV-1 and KCVPBCV-1 L94H tend to the same upper and lower plateaus. This is most evident at low $\mathrm{H}^{+}$concentrations ( $\mathrm{pH}$ 9). For $\mathrm{pH} \leq 4$ more data points are necessary to confirm this common conductance limit. However, it already seems as if the minimal conductance values for the wild type channel and the mutant KCVPBCV-1 L94H lie within the same range. This implies that the reducing effect on the unitary single channel conductance in KCVPBCV-1 and

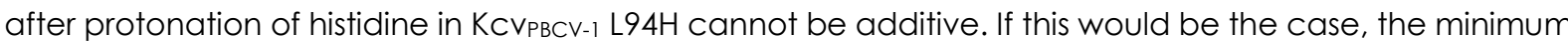
plateau should be significantly lower for KCVPBCV-1 $\mathrm{L} 94 \mathrm{H}$ than for KCVPBCV-1. Instead, the effect resulting from protonation of $\mathrm{L} 94 \mathrm{H}$ seems to overwrite the effect of $\mathrm{pH}$ on the wild type channel.

In order to explain this phenomenon quantitatively, a two-state model can be proposed. According to this model (fig. $30 \mathrm{~A}$ ) both, $\mathrm{KCV}_{\mathrm{PBCV} \text {-1 }}$ and KCVPBCV-1 L94H, exist in a state $\mathrm{S}_{1}$ with a maximal conductance ( $\left.G_{\max }\right)$ or a state $S_{2}$ with a minimal conductance $\left(G_{\min }\right)$. Without the histidine in the wild type channel, there is only one possible way for $\mathrm{KCV}_{\mathrm{PBCV}-1}$ to pass from $\mathrm{S}_{1}$ to $\mathrm{S}_{2}$. This reversible transition is characterized by the association rate constant $\mathrm{k}_{1}$ and the dissociation rate constant k-1. After mutation of L94H a second pathway is generated that is characterized by the association rate constant $k_{2}$ and the dissociation rate constant k-2. The association constants $\mathrm{k}_{1}$ and $\mathrm{k}_{2}$ are dependent on both, the proton concentration $\left[\mathrm{H}^{+}\right]$ and the Hill coefficients $h_{1}$ and $h_{2}$ as follows:

$\mathrm{k}_{1}=\mathrm{k}_{1}^{*} \times\left[\mathrm{H}^{+}\right]^{\mathrm{h}_{1}}$
$\mathrm{k}_{2}=\mathrm{k}_{2}^{*} \times\left[\mathrm{H}^{+}\right]^{\mathrm{h}_{2}}$

with $k_{1}^{*}$ and $k_{2}^{*}$ being the rate constants for $\left[H^{+}\right]^{h 1}=1 \mathrm{M}^{h 1}$ and $\left[H^{+}\right]^{h 2}=1 \mathrm{M}^{\mathrm{h} 2}$, respectively. It can now be assumed that the conductance $\mathrm{G}$, which is measured at a certain $\mathrm{pH}$ value, is given by 
$G=P\left(S_{1}\right) \times G_{\text {max }}+P\left(S_{2}\right) \times G_{\text {min }}$

with $P\left(S_{1}\right)$ and $P\left(S_{2}\right)$ being the occupation probabilities of state $S_{1}$ and state $S_{2}$. According to the two-state model (fig. $30 \mathrm{~A}), P\left(S_{1}\right)$ can be calculated under equilibrium conditions $(d P(S 1) / d t=d P(S 2 / d t)=0)$ as follows:

$P\left(S_{1}\right)=\frac{k_{-1}+k_{-2}}{k_{-1}+k_{-2}+k_{1}^{*} \times\left[H^{+}\right]^{n_{1}}+k_{2}^{*} \times\left[H^{+}\right]^{h_{2}}}$

With the constraint $P\left(S_{1}\right)+P\left(S_{2}\right)=1$ follows for $G$ :

$$
\begin{aligned}
& G=P\left(S_{1}\right) \times G_{\text {max }}+\left(1-P\left(S_{1}\right)\right) \times G_{\text {min }}=P\left(S_{1}\right) \times\left(G_{\text {max }}-G_{\text {min }}\right)+G_{\text {min }} \\
& G_{L_{94 H}}=G_{\text {min }}+\left(G_{\text {max }}-G_{\text {min }}\right) \times \frac{1}{1+\frac{k_{1}^{*}}{k_{-1}+k_{-2}} \times\left[H^{+}\right]^{h_{1}}+\frac{k_{2}^{*}}{k_{-1}+k_{-2}} \times\left[H^{+}\right]^{h_{2}}}
\end{aligned}
$$

For the WT channel this equation becomes:

$$
G_{w t}=G_{\text {min }}+\left(G_{\max }-G_{\min }\right) \times \frac{1}{1+\frac{k_{1}^{*}}{k_{-1}} \times\left[H^{+}\right]^{h_{1}}}
$$

In order to find a single set of parameters that describes the effect of $\mathrm{pH}$ on the conductance of both, $K_{C C V_{P B C V}-1}$ and KCVPBCV-1 L94H, a joint fit was performed. In this fit the measured values of the conductance of the wild type channel were fitted with equation (11), while the values recorded for the mutant channel were fitted with equation (10). The best fits are shown as dashed lines in fig. 30 B (blue: KCVPBCV-1; red: KCVPBCV-1 L94H). The parameters yielded by the joint fit are listed in Table 2 (appendix). Using these parameters and

$$
G_{\text {His }}=G_{\text {min }}+\left(G_{\text {max }}-G_{\text {min }}\right) \times \frac{1}{1+\frac{k_{2}^{*}}{k_{-2}} \times\left[H^{+}\right]^{h_{2}}}
$$

it is possible to calculate the effect of protonation of $\mathrm{L} 94 \mathrm{H}$ on the unitary single channel conductance after eliminating the background effect on the wild type channel. The corresponding simulated data is shown in fig. $30 \mathrm{~B}$ (black dashed line). Noticeably, the resulting curve is virtually identical to the fitted data of $\mathrm{KCV}_{\mathrm{PBCV}} \mathrm{-1} \mathrm{L} 94 \mathrm{H}$. This is due to the much faster kinetics of the protonation/deprotonation of $\mathrm{H} 94$ compared to the rate constants $k_{1}$ and $k_{-1}$. 
From the joint fit of the data, which is presented in fig. $30 \mathrm{~B}$, absolute values for $k_{1}^{*}, k_{-1}, k_{2}^{*}$, and $k_{-2}$ are provided. However, these values are not trustworthy, because $k_{1}^{*} \cdot\left[H^{+}\right]^{h 1}$ and $k_{-1}$ are several orders of magnitude smaller than $k_{2}^{*} \cdot\left[H^{+}\right]^{h 2}$ and $k_{-2}$. Therefore, only the ratios $K_{1}^{*}=\frac{k_{1}^{*}}{k_{-1}}$ and $K_{2}^{*}=\frac{k_{2}^{*}}{k_{-2}}$ are well defined. This can be demonstrated by calculating the weighted square error sum $\chi^{2}$ for different sets of $k_{1}^{*}, k_{-1}, k_{2}^{*}$, and $k_{-2}$ (fig. 31 ).

The data was fitted by minimizing $\chi^{2}$ (equation 3). Fig. 31 shows the change of $\chi^{2}$ after modification of either both rate constants $k_{1}$ and $k_{-1}$ or k2 and k-2, respectively, by multiplication with a factor 10y, leaving the ratio unaltered (fig. $31 \mathrm{~A}$ ) or after changing the ratio of $k_{1} / k_{-1}$ and $k_{2} / k_{-2}$ by multiplying only $k_{1}$ and $k_{2}$ with a factor $10^{y}$ (fig. 31 B). Since $k_{2} / k_{-2}$ has a much faster kinetic and the values are several orders of magnitude larger than $k_{1} / k_{-1}$, equation (10) is determined by the histidine effect. By multiplying both, $k_{1}$ and $k_{-1}$ with factors $10 y$ with $y \leq 0$, no change in $\chi^{2}$ is seen, since the difference between the ratios of $k_{1} / k_{-}$ 1 and $k_{2} / k_{-2}$ are further increasing. After multiplication with factors $10 y$ with $y>0, \chi^{2}$ steadily increases, since the absolute values of $k_{1}$ and $k_{-1}$ approach the values of $k_{2}$ and k-2. An inversed effect of $\chi^{2}$ is seen for multiplication of $k_{2}$ and $k_{-2}$ with the same factors. If all rate constants are multiplied with the same factors, no difference of $\chi^{2}$ can be seen (fig. 31 A, black line). This can be expected, because for the last case the rate constants $k_{2}$ and $k_{-2}$ is always several orders of magnitude higher than $k_{1}$ and $k_{-1}$.

On the other hand, multiplication of only $k_{1}$ and $k_{2}$ with a factor $10 y$ (fig. 31 B) changes the ratios of $k_{1} / k_{-1}$ and $k_{2} / k_{-}$. This leads to a drastic increase of $\chi^{2}$ for all factors $10^{y}$ except for $y=0$. Together, these results demonstrate that the model presented in fig. $30 \mathrm{~A}$ is insensitive towards changes in $k_{1}$ and $k_{-1}$ as well as $k_{2}$ and $k_{-2}$ as long as the ratios $k_{1} / k_{-1}$ and $k_{2} / k_{-2}$ are not changed.

Consequently, instead of the rate constants, it is better to consider only the apparent dissociation constants $K_{d, w t}^{a p p}, K_{d, H i s^{\prime}}^{a p p}$ and $K_{d, L 94 H}^{a p p}$ :

$\mathrm{K}_{\mathrm{d}, \mathrm{wt}}^{\mathrm{app}}=\frac{\mathrm{k}_{-1}}{\mathrm{~K}_{1}^{*} \times\left[\mathrm{H}^{+}\right]^{\mathrm{h}_{1}}}$

$\mathrm{K}_{\mathrm{d}, \mathrm{His}}^{\mathrm{app}}=\frac{\mathrm{K}_{-2}}{\mathrm{~K}_{2}^{*} \times\left[\mathrm{H}^{+}\right]^{\mathrm{h}_{2}}}$

$\mathrm{K}_{\mathrm{d}, \mathrm{L}, 4 \mathrm{H}}^{\mathrm{app}}=\frac{\mathrm{k}_{-1}+\mathrm{k}_{-2}}{\mathrm{k}_{1}^{*} \times\left[\mathrm{H}^{+}\right]^{\mathrm{h}_{1}}+\mathrm{k}_{2}^{*} \times\left[\mathrm{H}^{+}\right]^{\mathrm{h}_{2}}}$

The dissociation constant is a measure for the propensity to find the system in the deprotonated state $S_{1}$ under equilibrium conditions. The following relationships apply for $K$ and the occupation probabilities $P\left(S_{1}\right)$ and $\mathrm{P}\left(\mathrm{S}_{2}\right): K>1 \Rightarrow P\left(S_{1}\right)>P\left(S_{2}\right), K<1 \Rightarrow P\left(S_{1}\right)<P\left(S_{2}\right)$, and $K=1 \Rightarrow P\left(S_{1}\right)=P\left(S_{2}\right)=0.5$.

The $\left[\mathrm{H}^{+}\right]$-dependence of the dissociation constants is shown in fig. $30 \mathrm{C}$. The line for the solely effect caused by the mutation to histidine (black dashed line) lies, as is to be expected, on top of the line representing both effects (red). The slope of the straight lines represents the hill coefficient. From the 
steepness of the lines a stronger $\left[\mathrm{H}^{+}\right]$dependence can be recognized for the histidine effect and thus the effect on KCVPBCV-1 L94H.

A Wild type effect

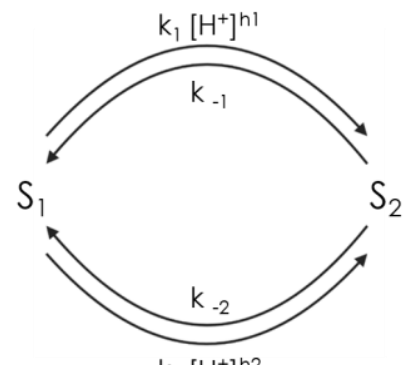

$\mathrm{k}_{2}\left[\mathrm{H}^{+}\right]^{\mathrm{h} 2}$

Histidine effect

B

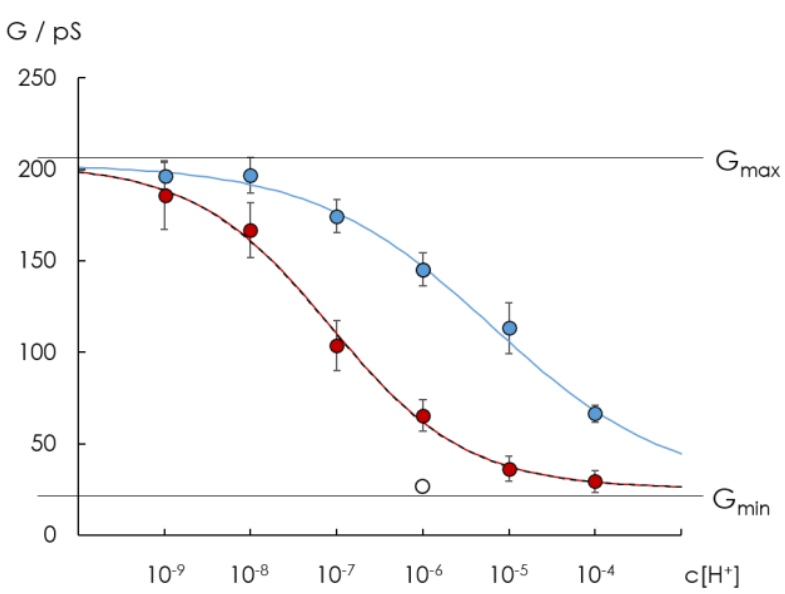

C

$$
K_{d} \text { app }
$$

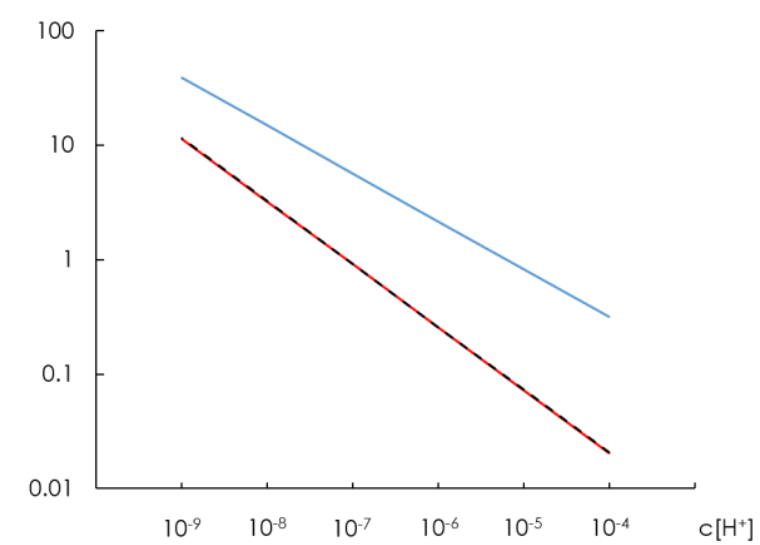

Figure 30 - Simple two-state model describes the effect of $\mathrm{pH}$ on KCVPBCV-1 and KCVPBCV-1 L94H. (A) Scematic illustration of the two-state model: The channel can switch between a state $S_{1}$ with a high conductance $\left(G_{\max }\right)$ and a state $S_{2}$ with a low conductance $\left(G_{\min }\right)$ via two different ways, which represent the effect of $\mathrm{pH}$ on the wild type channel KCVPBCV-1 and the effect caused by the protonation of histidine ( $\mathrm{KCVPBCV}-1 \mathrm{~L}$ 94H). The rate constants that determine the transition are defined as $\mathrm{k}_{1}, \mathrm{k}_{-1}, \mathrm{k}_{2}$ and $\mathrm{k}_{-2}$, while $\mathrm{k}_{1}$ and $\mathrm{k}_{2}$ are depending on $\mathrm{H}^{+}$concentration and the Hill-coefficient $\left(\mathrm{h}_{1}\right.$ and $\left.h_{2}\right) . K_{1}$ and $K_{2}$ represent the dissociation constants. (B) Unitary single channel conductance of KCVPBCV-1 (blue circles) and KCVPBCV-1 L94H (red circles) as a function of $\mathrm{H}^{+}$concentration, fitted with equation (11) and equation (10) from the two-state model, respectively. Gmax and $G_{\min }$ are indicated with black lines. The solely effect caused through mutation $\mathrm{L} 94 \mathrm{H}$ was calculated from the model and is marked with a black dashed line. (C) Corresponding $K_{d}^{a p p}$ values are calculated with equations (13), (14) and (15) from (B). 
A

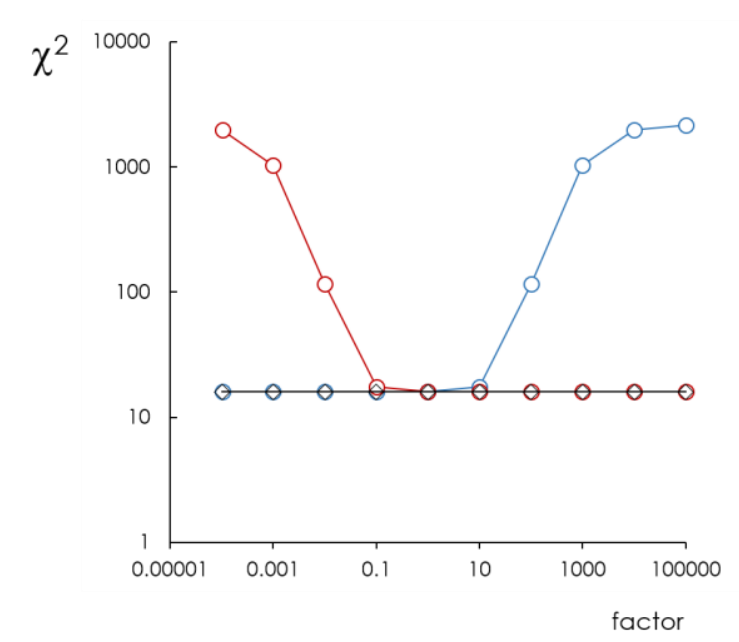

B

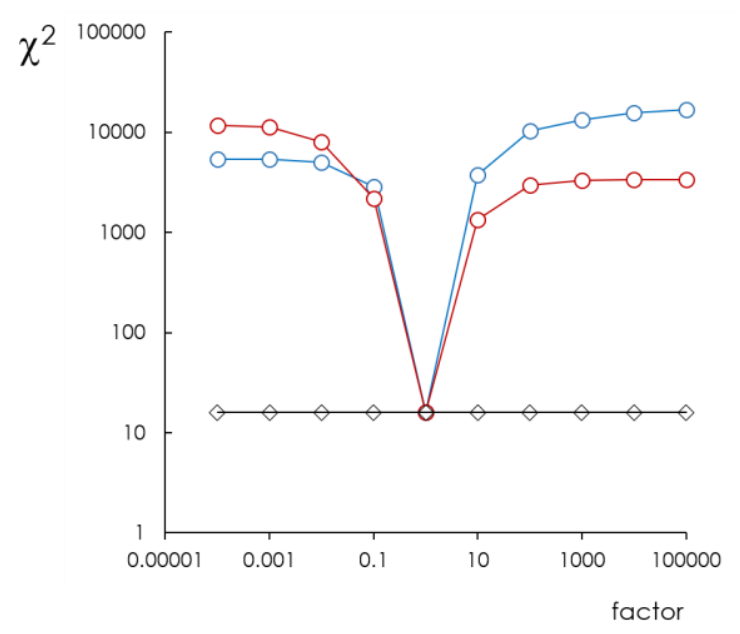

Figure 31 - Variation of $\chi^{2}$ after modification of the rate constants $\mathbf{k}_{1}, \mathbf{k}_{-1}, \mathbf{k}_{2}$ and $\mathbf{k}_{-2}$. Multiplication of $k_{1}, k_{-}$ $1, k_{2}$ and $k_{-2}$ with a factor 10y. (A) $\chi^{2}$ after multiplying $k_{1}$ and $k_{-1}$ (blue), $k_{2}$ and $k_{-2}$ (red) and $k_{1}, k_{-1}, k_{2}$ and $k_{-2}$ (black) with a factor $10 y$, without changing the ratios of $k_{1} / k_{-1}$ and $k_{2} / k_{-2}$ (B) $\chi^{2}$ after changing the ratios of $k_{1} / k_{-1}$ and $k_{2} / k_{-2}$ by multiplication of $k_{1}$ (blue) or $k_{2}$ (red) with a factor $10 y$. Additionally, $k_{1}, k_{-1}, k_{2}$ and $k_{-2}$ (black) after multiplication of the respective rate constant with a factor $10^{y}$.

In this simple model it must be considered that with four different subunits there are four different positions within the channel that can be affected by $\mathrm{H}^{+}$. In reality the system is even more complex. In addition to the possibility of all four subunits being protonated or deprotonated there are at least four more possibilities: protonation of one, two adjacent or opposite, or three subunits. The conducting state $G$ represents the mean current of all possible states. Regardless of which state the channel is in, it can be said with certainty that a positive charge at the intracellular entrance to KCVPBCV-1 has a negative impact on the unitary single channel conductance. A confirmation of this hypothesis comes from experiments in which $\mathrm{NiCl}_{2}$ was added to $\mathrm{KCVPBCV}_{-1} \mathrm{~L} 94 \mathrm{H}$ at $\mathrm{pH}$ 9. At such low $\mathrm{H}^{+}$concentrations, the probability of all four subunits being deprotonated is close to unity. Histidine is known to coordinate $\mathrm{Ni}^{2+}$ in its deprotonated state. Introducing the doubly positively charge from $\mathrm{Ni}^{2+}$ has shown to create different sublevels with lower conductances (fig. 24). Examination of the single channel traces of $\mathrm{KCVPBCV}_{-1} \mathrm{~L} 94 \mathrm{H}$ at pH 9 with $\mathrm{NiCl}_{2}$ and KCVPBCV-1 L94H at pH 6 has a very similar effect, which is illustrated in fig. 32 . With $10 \mu \mathrm{M} \mathrm{NiCl}_{2}$ the channel shows no longer one distinct open level, but multiple sublevels with a reduced single channel conductance. Three maxima can be identified in the histogram (fig. 24), with an even distribution of data points within the three states. Raising the $\mathrm{NiCl}_{2}$ concentration to $100 \mu \mathrm{M} \mathrm{NiCl} 2$ causes an increased frequency of the channel in the state with the smallest conductance. With more $\mathrm{Ni}^{2+}$ present, the probability of histidine to coordinate $\mathrm{Ni}^{2+}$ increases. 


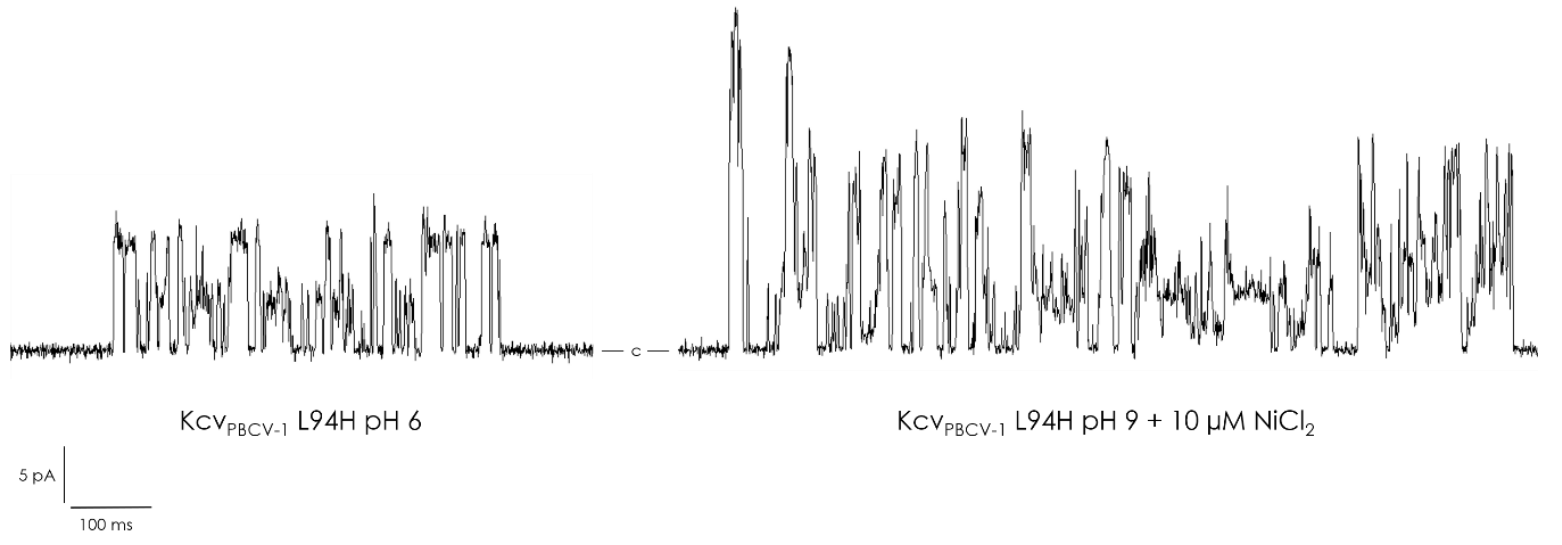

Figure 32 - Gating of KCVPBCV-1 $\mathbf{L} 94 \mathrm{H}$ at $\mathrm{pH}$ 6, and pH 9 after addition of $10 \mu \mathrm{M} \mathrm{NiCl}$. Two exemplary single channel traces are compared: KCVPBCV-1 L94H at pH 6 (left) and KCVPBCV-1 L94H at pH 9, after addition of $10 \mu \mathrm{M} \mathrm{NiCl}_{2}$. $\mathrm{C}$ marks the closed level. After addition of $\mathrm{NiCl}_{2}, \mathrm{KCV}_{\mathrm{PBCV}}-1 \mathrm{~L}$ 94H shows similar gating sublevels as can be seen for KCVPBCV-1 L94H at ph 6. Measurements were performed under symmetrical conditions with $100 \mathrm{mM} \mathrm{KCl}$ and $10 \mathrm{mM}$ buffer (pH $6:$ MES; pH 9: TAPS). For better visualization, data was subsequently filtered with $500 \mathrm{~Hz}$.

It remains unclear in what way $\mathrm{H}^{+}$has an impact on the conductance of KCVPBCV-1 L94H. A weak $\mathrm{H}^{+}$ dependency can already be observed for KCVPBCV-1. A possible explanation might be an effect of $\mathrm{H}^{+}$on the membrane. The possibility of the head group of DPhPC to interact with $\mathrm{H}^{+}$through protonation is unlikely, since the pKa value for the phosphate group is 1 (Marsh, 1990). However, it cannot be ruled out that $\mathrm{H}^{+}$interacts with the phospholipids or influences the membrane in some other, unknown way. Another option might be a blocking effect caused by the $\mathrm{H}^{+}$ions with decreasing $\mathrm{pH}$ values. Increasing the $\mathrm{H}^{+}$ concentrations might lead to a competitive effect between $\mathrm{K}^{+}$and $\mathrm{H}^{+}$, since the probability of $\mathrm{H}^{+}$ions being near the entrance to the SF is significantly higher.

Mutation of $\mathrm{L94H}$ introduces four possible residues that can be protonated. With every protonated histidine per subunit, an additional positive charge is present near the intracellular entrance of the pore. This most likely has an effect on the local $\mathrm{K}^{+}$concentration in the area of the intracellular gate. It is reasonable to assume that additional positive charges will have a repelling effect on the $\mathrm{K}^{+}$ions in the solution, causing a reduced ion flow through the channel pore.

Furthermore, additional charges in this region of the channel could also interfere with the intracellular gate. It has been mentioned before that a constant formation and disruption of salt bridges between the free negative charge of the $\mathrm{C}$-terminus and the positively charged amino acids within the $\mathrm{N}$-terminus determines channel gating (Hertel et al., 2010, Tayefeh et al., 2009). Upon disruption, $\mathrm{K}^{+}$ions can bind to the now free accessible negative charge of the C-terminus, which blocks the entrance/exit of further ions. The introduction of further positive charges might lead to conformational changes which interfere with the critical salt bridge network. Protonation of the carboxyl groups can be ruled out, since the pKa value for this reaction lies by 2 . 
Protonation of all four subunits within $\mathrm{KCVPBCV}-1 \mathrm{L94H}$ seems to have a stabilizing effect on channel conductance. This view is confirmed by measurements in the range of $\mathrm{pH} 4$ and $\mathrm{pH}$ 6. While the wild type channel already shows a clear increase of the conductance between pH 4 and 5, for KCVPBCV-1 L94H the conductance level hardly changes. Only from pH 6 onward a clear change in gating can be seen. However, the lower conductance level is still on the same level as for $\leq \mathrm{pH} 5$. This indicates that all four subunits of KCVPBCV-1 $\mathrm{L} 94 \mathrm{H}$ are protonated in the $\mathrm{pH}$ window between $\mathrm{pH} 4$ and $\mathrm{pH} \sim 6$. Constant protonation and deprotonation of various subunits might lead to a permanent switching between different levels of conductance at $\mathrm{pH}$ 6. Because the conductance level of all four protonated subunits is stable, this level is distinct and easy to identify. Deprotonation of one or more subunits might result in different levels of conductance, which would explain the different levels seen at pH 6 (fig. 22 A, within the dotted box). With decreasing $\left[\mathrm{H}^{+}\right]$, there is still a possibility that one or more of the four histidines in the tetramer are stochastically protonated. This could explain the occasional bursts that can be seen at $\mathrm{pH}$ $\geq 7$ (fig. 22 A). During these bursts no defined open state can be identified, which is consistent with the idea that the protein is instable when the four histidines are only partially protonated.

Further experiments are necessary to confirm the hypothesis of the simple two state model. The range of $\mathrm{pH}$ has to be further extended. This is especially important for the range below $\mathrm{pH} 4$, to confirm if KCVPBCV-

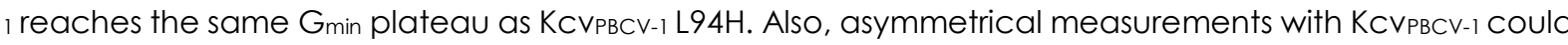
help understand the effect $\mathrm{pH}$ has on the wild type channel.

While the unitary single channel conductance of $\mathrm{KCVPBCV}_{-1}$ is $\mathrm{pH}$ sensitive, there is no obvious impact on the open probability. This suggests that the mutation of $\mathrm{L} 94 \mathrm{H}$ has two independent effects. This hypothesis is supported by the fact that at $\mathrm{pH} 7 \mathrm{KCV}_{\mathrm{PBCV}-1} \mathrm{~L} 94 \mathrm{H}$ shows only a reduction of the unitary single channel conductance, while the open probability has still the voltage dependency of the wild type. Merely at $\mathrm{pH}$ 4 a similar shift of the voltage dependency can be seen for both, KCVPBCV-1 and KCVPBCV-1 L94H. However, all other measurements of KCVPBCV-1 at different $\mathrm{pH}$ have no such shift but possess the same voltage dependency that appears at $\mathrm{pH}$ 7. On the contrary, KCVPBCV-1 L94H shows a steady shift of the voltage dependency with decreasing $\mathrm{H}^{+}$concentrations. An exception is seen for $\mathrm{KCV}_{\mathrm{PBCV}-1}$ at $\mathrm{pH}$. Five independent measurements could be assigned to two different gating types. Three measurements belonged to type 1, in which the voltage dependency is like at $\mathrm{pH}$ 7. For the other two measurements that belong to type 2 , the effect of the inverted voltage dependency seen at $\mathrm{pH} 4$ reoccurs. Interestingly, a second gating type was already observed for BLINK 1 . BLINK 1 is a synthetic blue-light-induced $\mathrm{K}^{+}$channel (see chapter 3.1.2). In this channel KCVPBCV-1 forms the pore module. When measured in PLBs with the same $\mathrm{K}^{+}$concentration (100 mM), but with $\mathrm{pH} 7$ instead of $\mathrm{pH} 8$, also two different gating types could be observed (fig. 11). These are very similar to the two gating types seen for KCVPBCV-1 at pH 8. Comparison of the two gating types (fig. 33) shows that the open probability for type 1 of BLINK 1 and KCVPBCV-1 at pH 8 is in the same range. It is possible that a decrease of the $\mathrm{H}^{+}$concentration results in the appearance of a second gating type of $\mathrm{KCV}_{\mathrm{PBCV}-1}$ that was evoked by modification of the N-terminus in KCVPBCV-1 for the creation of BLINK 1 . 


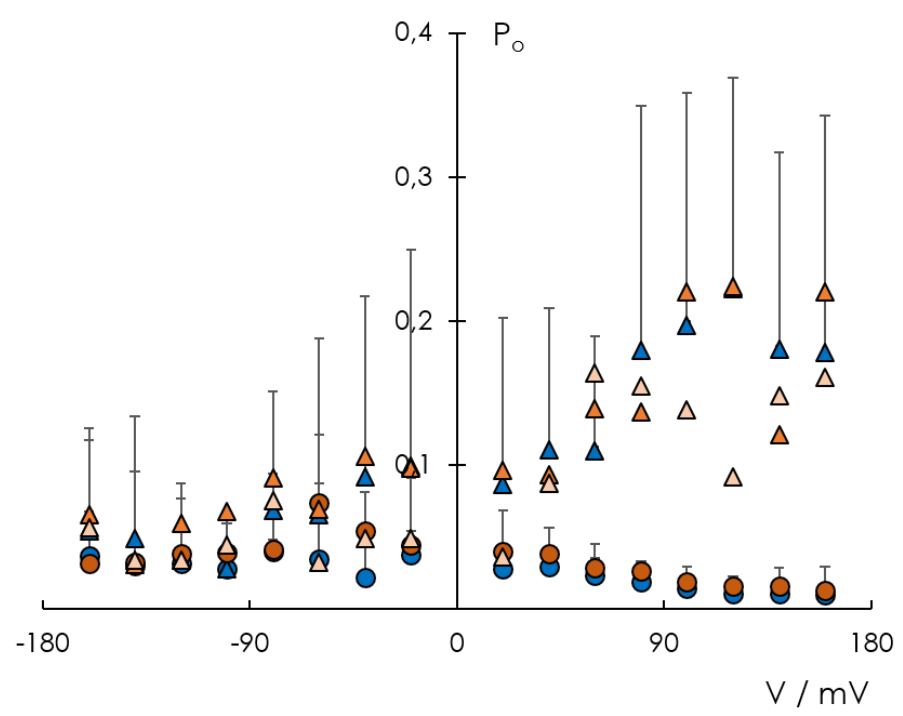

Figure 33 - Comparison of the different gating types of BLINK1 and KCVPBCV-1 at pH 8. Open probability of BLINK 1 (blue, $\mathrm{pH}$ 7) and $\mathrm{KCVPBCV}_{-1}$ (orange, $\mathrm{pH}$ 8). Both channels show two different gating types (type 1: triangles, type 2: circles). Data are mean values \pm standard deviation for $n$ independent measurements (BLINK1 type 1: $n=6$, type 2: $n=7 ; K_{C V P B C V}-1$ type 2: $n=3$ ). For KCVPBCV-1 type 1, two individual measurements are shown in two different shades of orange. Measurements were performed under symmetrical conditions with $100 \mathrm{mM} \mathrm{KCl}$ and $10 \mathrm{mM} \mathrm{HEPES}$ at $\mathrm{pH} 7$ for BLINK 1 and $100 \mathrm{mM} \mathrm{KCl}$ and $10 \mathrm{mM}$ HEPES at $\mathrm{pH} 8$ for KCVPBCV-1.

Regarding the $\mathrm{pH}$ dependet change in the open probability, it seems that the inversion of the voltage dependency is related to the introduction of a positive charge at the $\mathrm{C}$-terminus. In aqueous solutions at pH 7 lysine (Matthew et al., 1985; Zhu et al., 1995) and arginine (Nozaki \& Tanford, 1967; Li et al., 2008) are protonated, thus a positive charge is present. Histidine on the other hand is most likely only partially protonated at $\mathrm{pH}$ 7. The different $\mathrm{pKa}$ values of the three amino acids might explain why the inversed voltage dependency is only seen for L94K and L94R, but not for L94H. It remains unknown, why KCVPBCV-1 L94H does not show an inversion of the open probability at $\mathrm{pH} 7$, but already induces a reduction of the unitary single channel conductance compared to the wild type. The present experiments were not sufficient to explain why there are two different effects on KCVPBCV-1 when leucine at position 94 is exchanged to histidine. Further experiments with KCVPBCV-1 L94K and KCVPBCV-1 L94R using higher pH values should lead to deprotonation of lysine and arginine. The results of these experiments will help to identify if the effect is histidine specific or can be reproduced for mutations to amino acids with basic side chains.

These results, together with the fact that an increase of conductance was seen for KCVPBCV-1 L94D and $\mathrm{KCVPBCV}_{-1}$ L94E, suggest that charge distribution at the C-terminal end plays an important role. Positive charges seem to reduce the unitary single channel conductance, while negative charges increase it. Experiments carried out as part of a different study, examining the effect of charged lipids on gating of $\mathrm{KC}_{\mathrm{PBCV}-1}$, support this theory. In measurements with bilayers that were composed of a 50/50 mixture of the neutral DPhPC and the negatively charged DPhPS, KCVPBCV-1 shows an increase in unitary single channel conductance and open probability (appendix, fig. 44). 
There are a wide range of known ion channels that are gated by protons (C. C. Chen et al., 1998; Doyle et al., 1998; Starkus et al., 2003). One of the most well-known is KcsA. It is active at pH values below 4 and inactivates at lower $\mathrm{H}^{+}$concentrations. This inactivation is due to the formation of a complex salt bridge and hydrogen-bonding network between TMI and TM2, which stabilizes the closed conformation (Thompson et al., 2008). In KCVPBCV-1, salt bridges also play an important role, as they are one part of the gating mechanism (Hertel et al., 2010; Tayefeh et al., 2009). Altering the charge distribution by varying the proton concentration could have an impact on either the salt bridge stability of the known gate consisting of salt bridges between the C-terminal end of the channel and the positive amino acids within the $\mathrm{N}$ terminus, or some other salt bridge and/or hydrogen-bond network within the protein that remains unknown.

\subsubsection{Conclusion}

The results illustrate the complexity of interactions within the protein, especially in the case of small channels such as $\mathrm{KCV}_{\mathrm{PBCV}-1}$. Since the structure of $\mathrm{KCV}_{\mathrm{PBCV}-1}$ is yet unknown, predictions can only be made based on homology models (Hoffgaard et al., 2015; Tayefeh et al., 2009). The constant exchange of information gained from experimental data with that of computational predictions may help improving future attempts to development an advanced and more accurate homology model of KCV $\mathrm{PBCV}_{\mathrm{B}}$. 


\subsection{The viral $\mathrm{K}^{+}$channels $\mathrm{KCV}_{\mathrm{GNLD}}$ and $\mathrm{KCV}_{\mathrm{MT} 325}$}

$\mathrm{KCV}_{\mathrm{PBCV}-1}$ is by far not the only small viral $\mathrm{K}^{+}$channel which resembles only the pore region of bigger and more complex $\mathrm{K}^{+}$channels, while still possessing all their essential, characteristic properties. KCVGNLD and KCVMT325 are two further examples. KCVGNLD was collected from a water sample of a lake in Sisimiut, Greenland. The virus from which it was isolated infects Chlorella SAG, a symbiont in Heliozoae species. So far channels isolated from Chlorella SAG were very similar in sequence and categorized as ATCV-1 like channels (Fitzgerald et al., 2007). With only 89 amino acids per subunit KCVGNLD has unexpectedly a higher sequence similarity to channels isolated from Chlorella Pbi (Siotto, 2017). Sequencing of the entire viral host genome by the Van Etten laboratory (Lincoln, Nebraska) has shown that it contains a mixture of genes, suggesting it to be a hybrid of SAG and Pbi viruses, which makes it from an evolutionary point of view very interesting (Siotto, 2017). Patch clamp measurements in HEK293 cells have shown that KCVGNLD generates a high $\mathrm{K}^{+}$conductance, with a tenfold higher selectivity for $\mathrm{K}^{+}$over $\mathrm{Na}^{+}$. Like other $\mathrm{Kcv}$ channels it can also be blocked by $\mathrm{Ba}^{2+}$ (Siotto, 2017). Due to the similarity of the $\mathrm{K}^{+}$and $\mathrm{Ba}^{2+}$ radius, $\mathrm{Ba}^{2+}$ is able to bind to the SF of $\mathrm{K}^{+}$channels (Piasta et al., 2011 ) and is a well-known $\mathrm{K}^{+}$channel blocker (Neyton \& Miller, 1988a, 1988b).

KCVMT325 was found in the Phycodnavirus MT325, which infects Chlorella Pbi. Even though it has 95 aa per subunit, an alignment of $\mathrm{KCV}_{\text {GNLD }}$ and $\mathrm{KCV}_{\mathrm{MT} 325}$ shows a high sequence similarity between the two channels, while differing significantly from the 94 aa KCVPBCV-1 (fig. 34). KCVGNLD and KCVMT325 both lack the $\mathrm{N}$-terminal slide helix that is present in KCV, while KCVGNLD has 3 and KCVMT325 10 additional amino acids at the $\mathrm{C}$-terminal end. Compared to other members of the Kcv family besides KCVGNLD, KcVMT325 only shows a 50\% amino acid identity (Plugge et al., 2000). KcVMT325 has shown to generate a functional, slightly outward rectifying $\mathrm{K}^{+}$current when expressed in oocytes (Gazzarrini et al., 2006), just like when expressed in HEK293 cells. The latter are also very similar to the high currents recorded from KCVGNLD expression in HEK293 cells (Siotto, 2017).

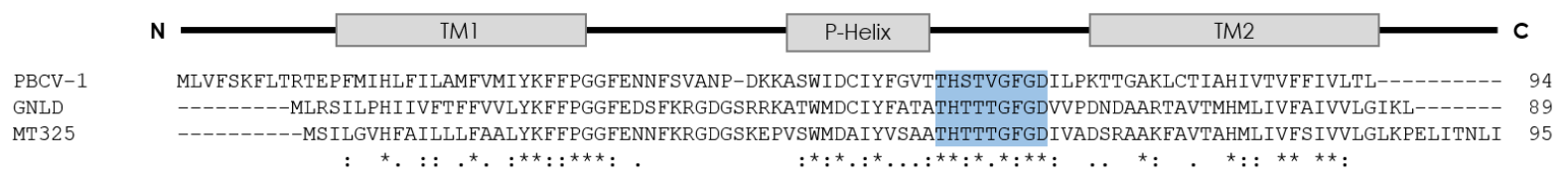

Figure 34 - Alignment of the viral $\mathrm{K}^{+}$channels KCVPBCV-1, KCVGNLD and KCVMT325. Transmembrane helices 1 and 2 (TM1 and TM2), as well as the pore helix (P-helix) are marked with grey boxes. The blue box highlights signature sequences of $\mathrm{K}^{+}$channels, which contains the GFG-motive (aligned with Clustal Omega; (Sievers et al., 2011 ; Madeira et al., 2019).

So far both channels have only been characterized macroscopically, either by patch clamp measurements in HEK293 cells (Siotto, 2017) or with two-electrode voltage clamp measurements in oocytes (Gazzarrini et al., 2006). To characterize the channels on a single channel level, the PLB method was applied. KCVMT325 and KCVGNLD have previously been cloned into the expression vector PEXP5$\mathrm{CT} / \mathrm{TOPO}$ and expressed in vitro in the presence of nanodiscs, as described in chapter 2.1.2. All measurements were performed under symmetrical conditions (10 mM HEPES, $\mathrm{pH}$ 7), with varying $\mathrm{K}^{+}$ 
concentrations of $50 \mathrm{mM}, 100 \mathrm{mM}, 250 \mathrm{mM}$ and $500 \mathrm{mM} \mathrm{KCl}$. Artificial membranes were exclusively made of DPhPC in all experiments. Protocols were applied from -180 mV to $+260 \mathrm{mV}$ in $20 \mathrm{mV}$ steps for $1 \mathrm{~min}$ per voltage.

Fig. 35 shows representative single channel traces of KCVGNLD (left) and KcVMT325 (right), for a $\mathrm{K}^{+}$ concentration of $100 \mathrm{mM}$ from $-180 \mathrm{mV}$ to $+180 \mathrm{mV}$ in $60 \mathrm{mV}$ steps. The raw data already show that both channels appear to have very similar properties, for both conductance and open probability. At negative voltages the KCV-characteristic flicker gating can be seen. This gating phenomenon is due to the very fast gating behavior of the channels, which cannot be completely resolved by the measuring device, appearing as an increasing noise with no distinct open states any more (Schroeder \& Hansen, 2006, 2007). In well-studied viral $\mathrm{K}^{+}$channels such as $\mathrm{KCVNTS}_{\mathrm{N}}$ or $\mathrm{KCVPBCV}_{-1}$, flicker gating appears at high negative voltages past - $60 \mathrm{mV}$ (Abenavoli et al., 2009; Schroeder, 2015; Rauh, 2018). For KCVGNLD and KCVMT325 this effect appears over the entire negative voltage range. Due to this fast gating and the high open probability, discrimination of a clear open and closed state at negative voltages is strongly hindered, unless the channel happens to show occasional longer closed events in the range of seconds (e.g. fig. 35 (A) KCVGNLD at - $180 \mathrm{mV}$ and (B) KCVMT325 $-60 \mathrm{mV},-120 \mathrm{mV},-180 \mathrm{mV}$ ). Due to the unpredictability of these longer closed events, pulse protocols were modified to end with 10 seconds at $0 \mathrm{mV}$, in order to catch a clearly defined closed state of the channel. At positive voltages flicker gating is not visible anymore. From $0 \mathrm{mV}$ to $+80 \mathrm{mV}$ the channel is almost exclusively in its open state, so an open probability of nearly $100 \%$ can be assumed. With increasing positive voltages $>80 \mathrm{mV}$, a decrease in the open probability can be seen, implying a voltage dependency. The uppermost trace shows a section of the one below at +180 $\mathrm{mV}$, but with a higher temporal resolution, for a better visibility of individual gating events. The better resolution shows that distinct opening and closing events still occur, but the amount and length of closed states increases, which is accompanied by a decrease of the open probability. While this voltagedependent decrease of the open probability seems similar for both channels, KCVGNLD has a slightly higher single channel conductance. At positive voltages, where the open and closed states of the channels are clearly distinguishable, also a different gating of KCVGNLD and KCVMT325 can be seen, which is shown in fig. 35 B. KCVGNLD can show episodes of high-conductive states (marked with red dotted lines), which are mostly short lasting in the ms range, but can also sometimes appear for a short amount of seconds. KCVMT325 on the other hand shows rare and short-lasting subconducting states (marked with red dotted lines). 


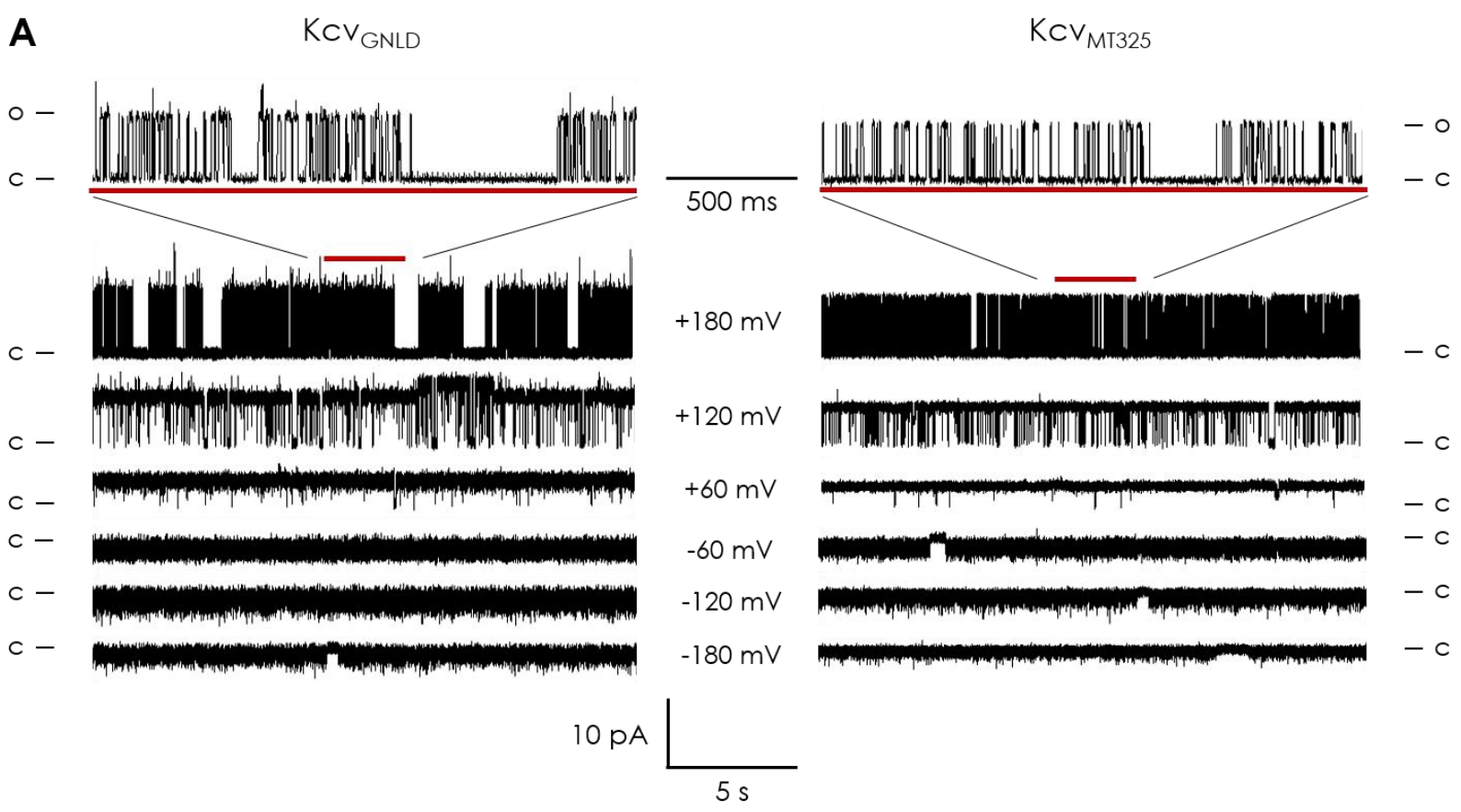

B $\quad \mathrm{KCV}_{\mathrm{GNLD}}$
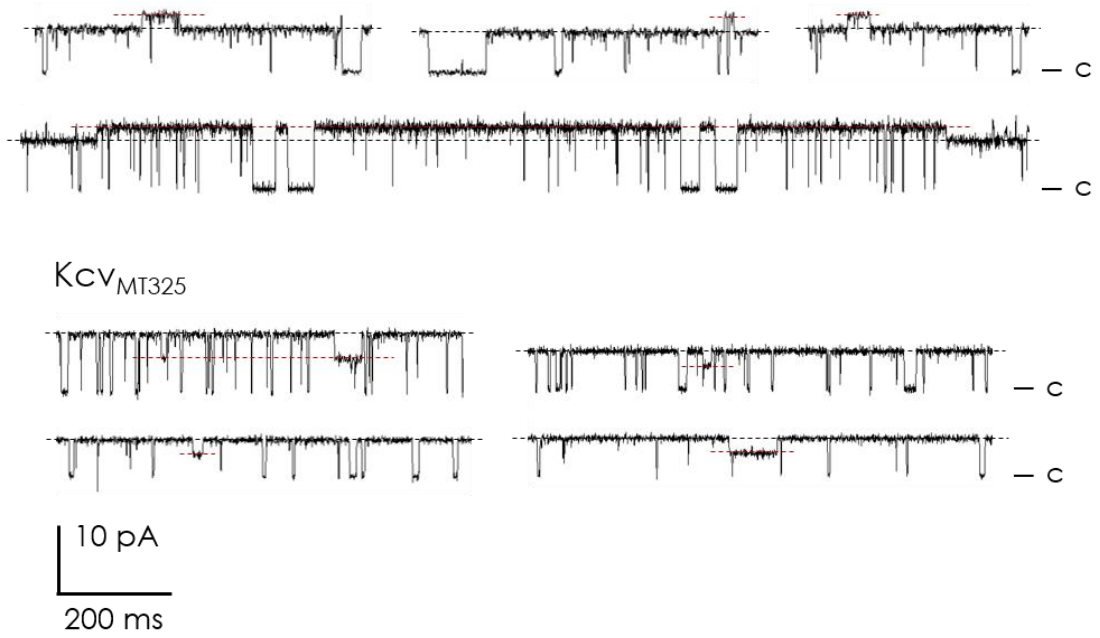

Figure 35 - Representative single channel traces for KcVGNLD and $\mathrm{KcV}_{\text {MT325. }}$. (A) Single channel traces of KCVGNLD (left) and KCVMT325 (right), from +180 mV to -180 mV in $60 \mathrm{mV}$ steps. The topmost traces show an enlarged section at $+180 \mathrm{mV}$ with a higher temporal resolution, indicated with a red bar. $\mathrm{c}$ and o mark the closed- and open state of the channels. (B) Excerpts from various measurements of KCVGNLD (top) and KCVMT325 (bottom) at $+120 \mathrm{mV}$. The main conductivity level is marked with black, while the high (GNLD) and low (MT325) conductivity levels are marked with red dotted lines. Measurements were performed under symmetrical conditions with $100 \mathrm{mM} \mathrm{KCl}, 10 \mathrm{mM} \mathrm{HEPES}$ at $\mathrm{pH}$ 7. For better visualization, data was subsequently filtered with $500 \mathrm{~Hz}$.

These first assumptions that were made based on analyses of the single channel traces can be confirmed by the corresponding i/V and Po curves. Fig. 36 shows the unitary single channel conductances of KCVGNLD (blue) and KcVMT325 (red) for $50 \mathrm{mM}, 100 \mathrm{mM}, 250 \mathrm{mM}$ and $500 \mathrm{mM} \mathrm{KCl}$, in which a decreasing color intensity corresponds to a decrease in $\mathrm{K}^{+}$concentration. At positive voltages both channels show an 
ohmic conductivity. For $100 \mathrm{mM} \mathrm{KCl}, \mathrm{KCV}$ GnLD has a single channel conductivity of $45.3 \pm 4.3 \mathrm{pS}$. The unitary conductance of $\mathrm{KCV}_{\mathrm{MT} 325}$ is with $34.8 \pm 1.4 \mathrm{pS}$ smaller. However, the conductance at negative voltages is much lower for both channels. Single channel traces in fig. 35 had already indicated that flicker gating seems to occur in the entire negative voltage range. Due to the fast gating of the channels, individual opening and closing events cannot be completely resolved by the measuring device. This appears as an increase in noise, resulting in a shift of the current distribution towards zero and a negative slope conductance in the resulting i/V curve (Schroeder \& Hansen, 2006, 2007). In order to obtain a clear open state in the histograms at negative voltages, current traces were filtered in KielPatch with a moving filter of 10 data points. This filter moves from one data point to the next across the entire measurement, calculating the mean value of a previously defined number of data points (in this case 10). Beta Fit analysis would be necessary, to see if the true current of the channels would continue in a linear manner, as it is known to happen for KCVNTS or KCVPBCV-1 (Abenavoli et al., 2009; Schroeder, 2015). The $\mathrm{K}^{+}$ concentration dependent single channel conductance of $K_{C V} V_{\text {GNLD }}$ and $K_{C V} \mathrm{MT}_{\mathrm{M} 325}$ are summarized in fig. 36 $\mathrm{C}$. The saturating increase in unitary conductance with increasing $\mathrm{K}^{+}$concentration for both channels can be fitted with the Michaelis-Menten equation:

$$
G=\frac{G_{\max } \cdot\left[K^{+}\right]}{K_{m}+\left[K^{+}\right]}
$$

It is used to describe enzyme kinetics to determine the half-maximal velocity $\left(\mathrm{K}_{\mathrm{m}}\right)$ and maximum velocity $\left(V_{\text {max }}\right)$, but can also be used to describe concentration-dependent ion permeation kinetics (Tolokh et al., 2005). In this case $\mathrm{K}_{m}$ describes the $\mathrm{K}^{+}$concentration for which a channel reaches its half-maximal conductivity. While both channels seem to converge slowly towards saturation, $G_{\max }$ is more than twice as high for KCVGNLD (273.4 pS) than for KCVMT325 (138.4 pS). The higher conductance of KCVGNLD was already indicated by the single channel traces in fig. 35 , but it becomes more clear with rising $\mathrm{K}^{+}$concentrations and additionally by comparison of the $\mathrm{Km}_{\mathrm{m}}$ value, which is nearly twofold higher for KCVGNLD (453.7 mM K+) compared to KCVMT325 $\left(263.7 \mathrm{mM} \mathrm{K}^{+}\right)$. 
A

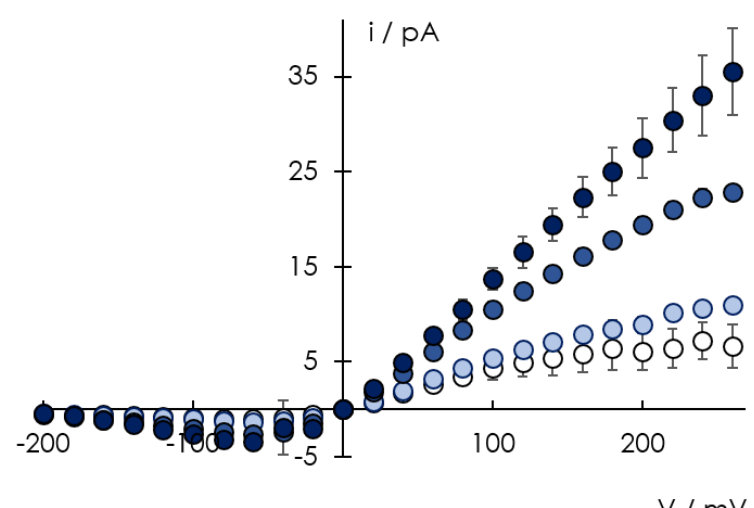

B

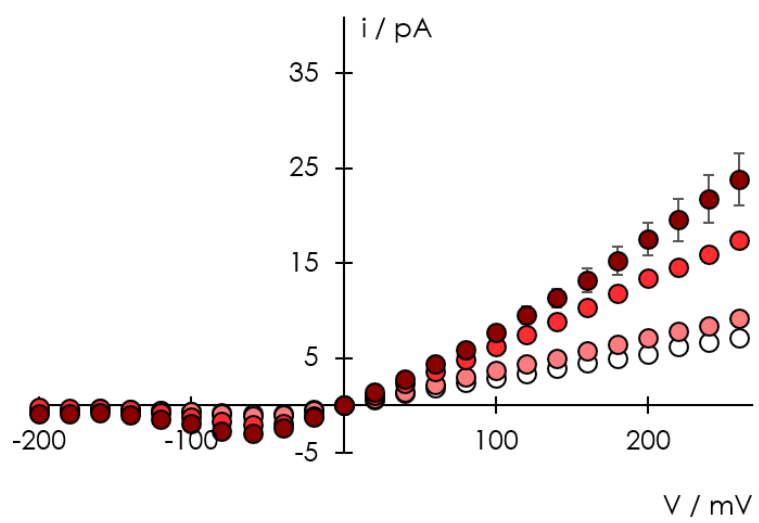

C

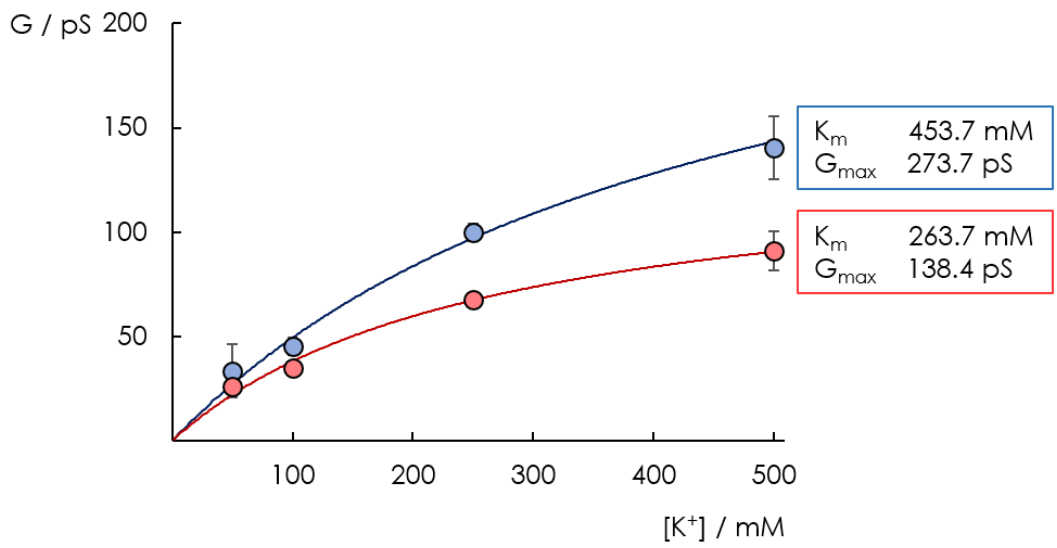

Figure $36-\mathrm{i} / \mathrm{V}$ curves and single channel conductance of $\mathrm{KCVGNLD}$ and $\mathrm{KCV}_{\mathrm{MT} 325}$ for different $\mathrm{K}^{+}$ concentrations. i/V curves for $\mathrm{KCV}_{\text {GNLD }}\left(\mathrm{A}\right.$, blue) and $\mathrm{KCV}_{\text {MT325 }}$ (B, red) in buffer with $500 \mathrm{mM}$ (dark), $250 \mathrm{mM}$ (medium), $100 \mathrm{mM}$ (light) and $50 \mathrm{mM}$ (white) $\mathrm{K}^{+}$. Data are mean values \pm standard deviation of $n$ independent measurements (KCVGNLD: $50 \mathrm{mM} \mathrm{KCl:} n=6,100 \mathrm{mM} \mathrm{KCl}: n=7,250 \mathrm{mM}$ KCl: $n=4,500$ mM KCl: $n=5$; MT325: 50 mM KCl: $n=8,100$ mM KCl: n=9, 250 mM KCl: $n=4,500$ mM KCl: $\mathrm{n}=4)$ (C) Single channel conductance of KCVGNLD (blue circles) and KCVMT325 (red circles) and corresponding fit (colored lines), with equation (16) yielding a $\mathrm{Km}_{\mathrm{m}}=453.7 \mathrm{mM} \mathrm{K}+$ for KCVGNLD and $\mathrm{Km}$ $=263.7 \mathrm{~K}^{+}$for $\mathrm{KCV}_{\mathrm{MT}} 325$. The $\mathrm{G}_{\max }$ values are $273.7 \mathrm{pS}$ for KCVGNLD and $138.4 \mathrm{pS}$ for KCVMT325. Measurements with varying $\mathrm{K}^{+}$concentrations were performed under symmetrical conditions with 10 mM HEPES at pH 7.

For high $\mathrm{K}^{+}$concentrations such as $500 \mathrm{mM} \mathrm{KCl}$, it is possible to distinguish the true current of KCVGNLD for negative voltages up to a certain point. Fig. 37 A shows exemplary single channel traces at $+80 \mathrm{mV}$ and $-80 \mathrm{mV}$ with different temporal resolutions. While it is no problem to differentiate between the open and closed state at positive voltages, it becomes ambiguous for negative voltages, which is apparent by looking at the enlarged section of fig. $37 \mathrm{~A}$ at $-80 \mathrm{mV}$ (bottom trace). The gating of the channel becomes so fast that it cannot be resolved any more by the measuring device. Within the red box an individual closing event is marked which was long enough to be properly resolved. Fig. $37 \mathrm{C}$ and $\mathrm{D}$ show the corresponding amplitude histogram of the single channel trace at $-80 \mathrm{mV}$, one with a moving filter of 10 applied by KielPatch (D) and one unfiltered (C). In both histograms the closed state is clearly visible, due 
to the modified pulse protocols which keeps the membrane at $0 \mathrm{mV}$ for 10 seconds at the end of every voltage. For lower $\mathrm{K}^{+}$concentrations, no defined peak could be seen to determine a distinct open state. For $500 \mathrm{mM} \mathrm{KCl}$, as can be seen in fig. $37 \mathrm{C}$, even though there is a great distribution of data points, still a small but defined peak at -7.8 pA is visible. This peak can be seen at negative voltages up to $-120 \mathrm{mV}$. When plotted in an i/V curve (fig. 37 B, grey circles), the conductance of KCVGNLD for negative voltages continues to be approximately in the same linear range as for positive voltages. The results of these experiments underscore that the negative slope conductance at negative voltages is an artifact of the recording system and caused by a very fast gating of the channels. For better comparison with the curves of lower $\mathrm{K}^{+}$concentrations and to stay consistent, current traces for negative voltages were routinely filtered with the KielPatch moving filter of 10. The resulting filtered histogram for $-80 \mathrm{mV}$ is shown in fig. 37 $D$. As a result of filtering, the peak that forms is more defined, but strongly shifted towards 0 and is now at -2.8 pA. The corresponding i/V curve is shown in fig. 37 B (black circles). The conductance of the channel is no longer linear, but the Kcv characteristic negative slope conductance can now be seen.

\section{A}
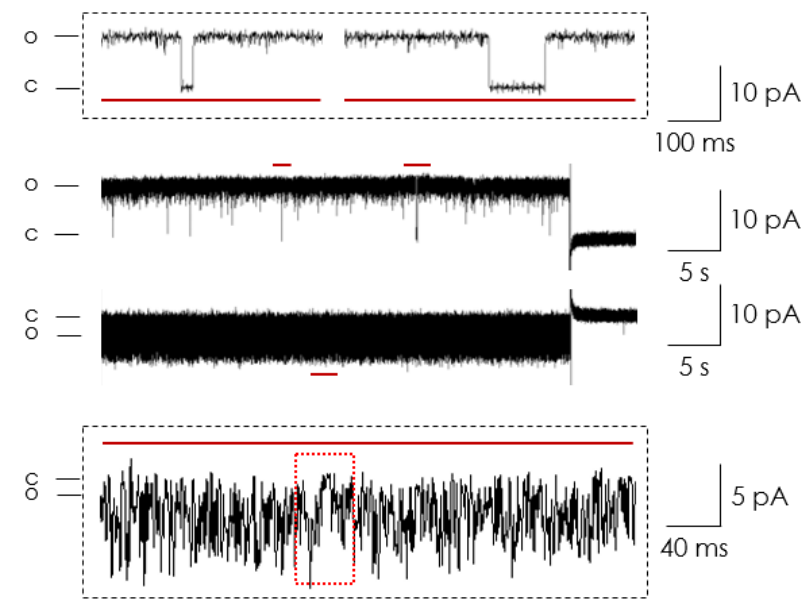

C

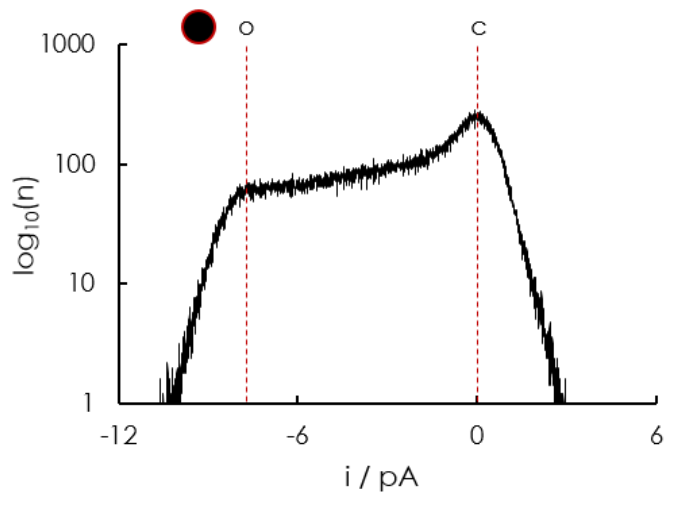

B

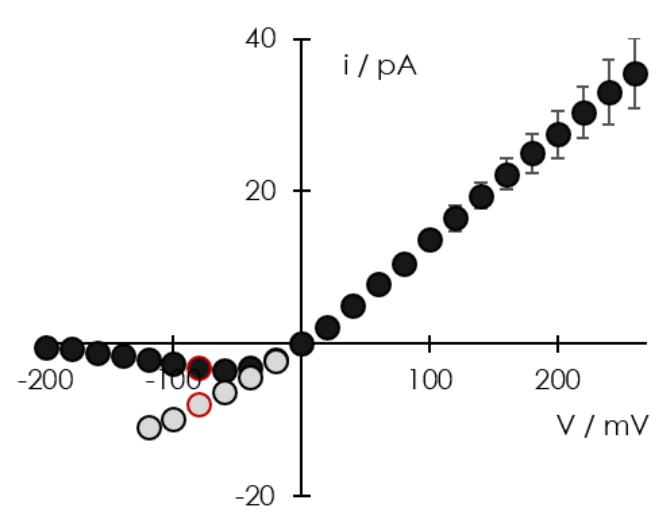

D

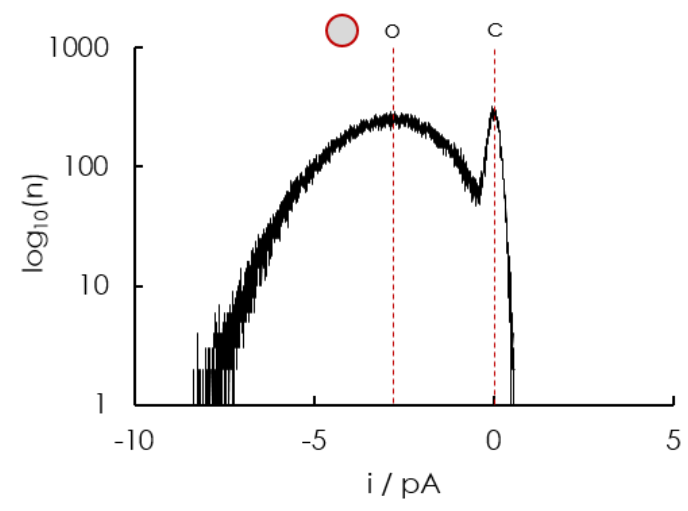


Figure 37 - Gating of KcVGNLD at negative voltages. (A) Representative single channel traces at $+80 \mathrm{mV}$ (two top traces) and $-80 \mathrm{mV}$ (two bottom traces). Data was collected within the dotted boxes with a higher temporal resolution allowing a better identification of closed events. $O$ and $c$ mark the open- and closed state. A distinct closing event at $-80 \mathrm{mV}$ is marked with the red dotted box. For better visualization, data was subsequently filtered with $500 \mathrm{~Hz}$. (B) i/V curve of KCVGNLD. For negative voltages the black circles represent the current after using a moving filter of 10 on the sampled data points. Unfiltered data are represented with grey circles. Data are mean values \pm standard deviation of $\mathrm{n}$ independent measurements $(\mathrm{n}=6)$. Corresponding amplitude histograms of unfiltered (C) and filtered (D) data was made at -80 $\mathrm{mV}$ and show the difference in the resulting current. Measurements were performed under symmetrical conditions, $500 \mathrm{mM} \mathrm{KCl}, 10 \mathrm{mM} \mathrm{HEPES}$, $\mathrm{pH} 7$.

While the conductance of $\mathrm{KCV}_{\text {GNLD }}$ and $\mathrm{KCV}_{\mathrm{MT}} 325$ differs in a $\mathrm{K}^{+}$concentration dependent manner, the voltage dependency of the open probability curves is more similar for both channels. Fig. 38 shows the voltage-dependent open probability of KCVGNLD (A, blue) and KcVMT325 (B, red) for 500 mM (dark), 250 mM (medium), $100 \mathrm{mM}$ (light) and $50 \mathrm{mM}$ (white) mM KCl. As already assumed based on the single channel traces in fig. 35 , both channels have a constant open probability of nearly $100 \%$ throughout the entire negative voltage range. Minor deviations can be seen for KCVGNLD, as a consequence of irregularly occurring closed events in the range of a few seconds. The open probability at positive voltages stays at nearly $100 \%$ up to a certain voltage where a steep decrease takes place, with a voltage-dependent drop of the open probability to up to less than $10 \%$. For both channels the voltage dependency shifts towards more positive voltages with an increasing $\mathrm{K}^{+}$concentration. It also appears that the plateau to which the open probability drops to is higher for increasing $\mathrm{K}^{+}$concentrations. While for $50 \mathrm{mM} \mathrm{KCl}$ the open probability drops to a value below 10\%, for $500 \mathrm{mM} \mathrm{KCl}$ it only drops down to $50 \%$ for KCV GNLD and $40 \%$ for KcVMT325, respectively. For each channel and $\mathrm{K}^{+}$concentration the individual curves were fitted with the Boltzmann equation:

$P_{\circ}=P_{\text {max }}+\frac{P_{\text {min }}-P_{\text {max }}}{1+e^{\frac{(z \cdot F) \cdot(V 1 / 2-V)}{R \cdot T}}}$

It describes the open probability $\left(P_{\circ}\right)$ as a function of the applied voltage $(V)$. $P_{\min }$ and $P_{\max }$ are the minimal and maximal value of the open probability, respectively. $V_{1 / 2}$ is the voltage at which the open probability is halfway between $P_{\max }$ and $P_{\min }$. $R$ is the universal gas constant, $T$ the temperature in ${ }^{\circ} \mathrm{K}, \mathrm{F}$ the Faraday constant and $z$ the valence of the ion. Experiments were performed at room temperature (25 ${ }^{\circ} \mathrm{C}$ ), so $\mathrm{RT} / \mathrm{zF}=26 \mathrm{mV} / \mathrm{z}$ and the equation can be simplified to

$$
P_{\circ}=P_{\max }+\frac{P_{\min }-P_{\max }}{1+e^{z \cdot \frac{V_{1 / 2-V}}{26 m V}}}
$$

The corresponding $\bigvee_{1 / 2}$ values were plotted as shown in fig. $38 \mathrm{C}$ and also fitted with the Michaelis-Menten equation (16) to determine $V_{\max }$ and $K_{m}$ for both channels. The curves for KCVGNLD and KCVMT325 are very similar, with the difference that $\mathrm{KCV}_{\text {MT325 }}$ reaches a slightly higher $\mathrm{V}_{\max }$ of $231.8 \mathrm{mV}$ compared to $\mathrm{KCV}_{\text {GNLD }}$ 
with $202.7 \mathrm{mV}$. Corresponding $\mathrm{K}_{m}$ values for both channels ( $\mathrm{Km} \mathrm{KcVGNLD}=30.1 \mathrm{mM} \mathrm{K}, \mathrm{Km}_{\mathrm{m}} \mathrm{K}_{\mathrm{m}} \mathrm{K}_{\text {MT325 }}=38.5$ $\mathrm{mM} \mathrm{K} \mathrm{K}^{+}$) are very similar.

A

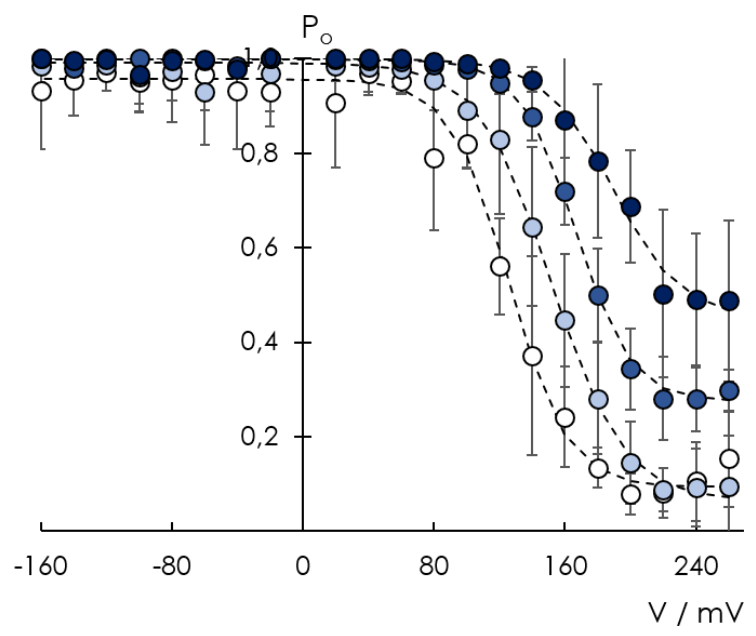

B

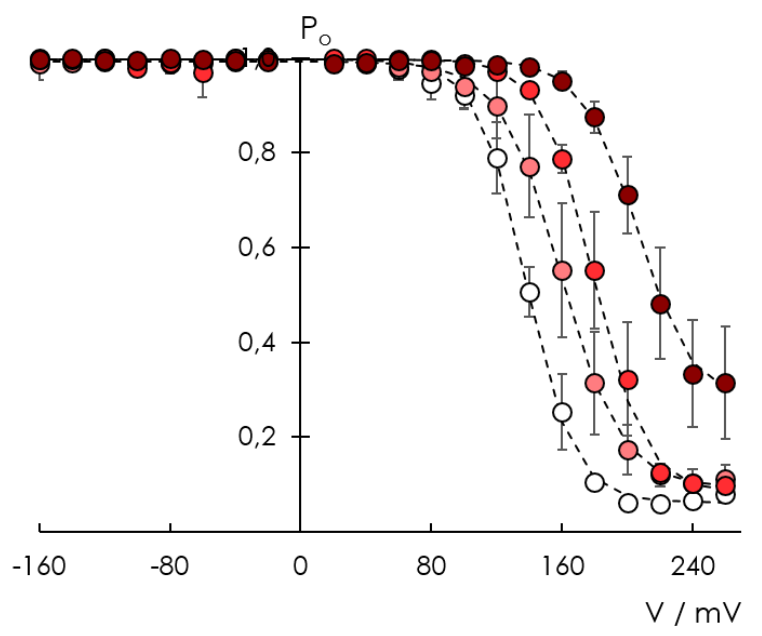

C

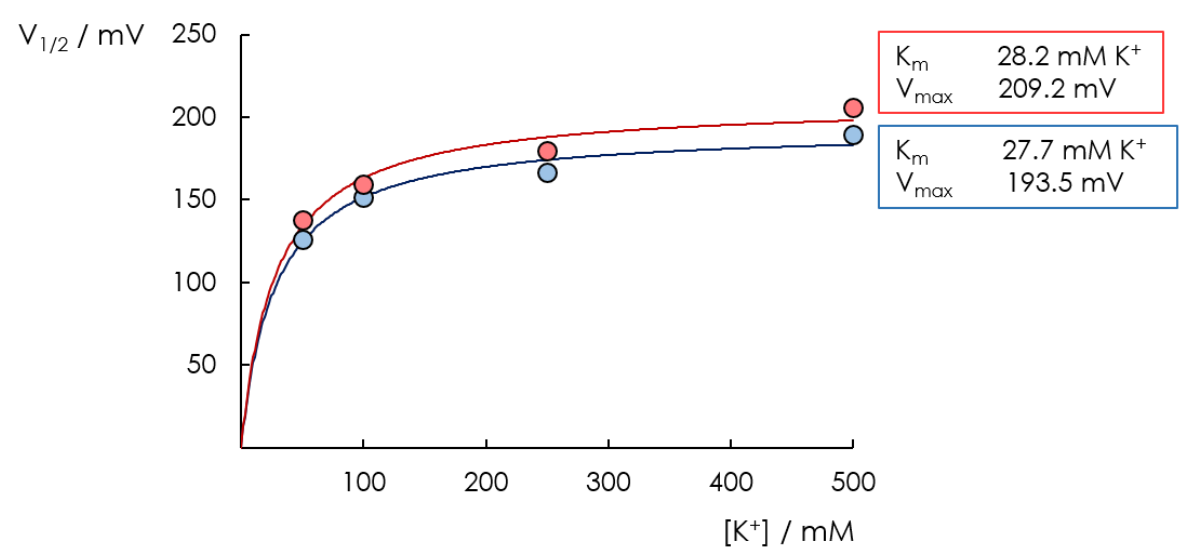

Figure 38 - Voltage-dependent open probability of KCVGNLD and $\mathrm{KCV}_{\mathrm{MT}} 25$ as a function of $\mathrm{K}^{+}$ concentrations. Open probability curves for $K_{C V G N L D}\left(A\right.$, blue) and $K_{C V M T 325}$ (B, red) for $500 \mathrm{mM}$ (dark), $250 \mathrm{mM}$ (medium), $100 \mathrm{mM}$ (light) and $50 \mathrm{mM}$ (white) $\mathrm{K}^{+}$. Data are mean values \pm standard deviation for $n$ independent measurements (GNLD: $50 \mathrm{mM} \mathrm{KCl}: \mathrm{n}=6,100 \mathrm{mM} \mathrm{KCl}: \mathrm{n}=7,250 \mathrm{mM} \mathrm{KCl}$ : $n=4,500$ mM KCl: n=5; MT325: 50 mM KCl: n=8, 100 mM KCl: n=9, 250 mM KCl: n=4, 500 mM KCl: $n=4)$. Corresponding Boltzman fits (from equation 18) are shown in black dashed lines. (C) $\bigvee_{1 / 2}$ for KCVGNLD (blue circles) and $\mathrm{KCV}_{\text {MT325 }}$ (red circles). Data was fitted with equation (16) yielding the following values: $V_{\max }=209.2 \mathrm{mV}$ for $K_{C V}$ MT325 and $V_{\max }=193.5 \mathrm{mV}$ for KCVGNLD, $\mathrm{Km}_{m}=28.2 \mathrm{mM} \mathrm{K}{ }^{+}$for KCVMT325 and $\mathrm{K}_{\mathrm{m}}=27.7 \mathrm{mM} \mathrm{K}+$ for $\mathrm{KCV}_{\mathrm{GNLD}}$. Measurements with varying $\mathrm{K}^{+}$concentrations were performed under symmetrical conditions with $10 \mathrm{mM}$ HEPES at $\mathrm{pH} 7$. 


\subsubsection{Mutation of threonine to valine right before the GFG motive eliminates voltage dependency in KCVGNLD and KCVMT325}

The ability to specifically conduct $\mathrm{K}^{+}$ions is determined by the SF of $\mathrm{K}^{+}$channels. Correspondingly, amino acid sequences which form the $\mathrm{SF}$ throughout $\mathrm{K}^{+}$channels found in all known forms of life are homologous (Doyle et al., 1998). Specifically, eight particular highly conserved amino acids are summarized as the signature sequence "TXXTXG(Y/F)G" (Heginbotham et al., 1994). The amino acids in these residues form the pathway for $\mathrm{K}^{+}$ions and are responsible for the high throughput with great precision. Several studies have shown that mutations within the SF are always accompanied by severe changes in channel properties (Heginbotham et al., 1994; Splitt et al., 2000; M. Zhou \& MacKinnon, 2004; Raja \& Vales, 2009); the respective proteins either lose their conductance or become non-selective.

Alignment of the signature sequence of several viral $\mathrm{K}^{+}$channels (fig. 39 A) shows that also within the sequences of these small viral channels the signature sequence is highly conserved. Interestingly, at position 5 of the signature sequence, $\mathrm{KCV}_{\text {GNLD }}$ and $\mathrm{KCV}_{\mathrm{MT}} 25$ possess a threonine, compared to the other aligned channels that have a valine in this position. Previous studies have shown that mutations at this homologous position within the bacterial $\mathrm{K}^{+}$channel $\mathrm{KcsA}$ or in a modified version of the Shaker $\mathrm{H}_{4} \mathrm{~K}^{+}$ channel lead to destabilization of the corresponding tetramer; also gating and selectivity properties of the channels were altered (Heginbotham et al., 1994; Raja \& Vales, 2009).

A

$K C V_{\text {PBCV-1 }}$
$\mathrm{KCV}_{\mathrm{GNLD}}$
$\mathrm{KCV}_{\mathrm{MT} 325}$
$\mathrm{KCV}_{\mathrm{NTS}}$
$\mathrm{KCV} \mathrm{V}_{\mathrm{S}}$
$\mathrm{KCV}_{\mathrm{NH}}$
$\mathrm{KCV}_{\mathrm{ATCV}-1}$

12345678

(60) THSTVGFG

(51) THTTTGFG

(50) THTTTGFG

(42) THTTVGYG

(42) THTTVGYG

(42) THTTVGYG

(42) THTTVGYG
B<smiles>CC(C)[C@H](N)C(=O)O</smiles><smiles>C[C@H](O)C(N)C(=O)O</smiles>

\section{L-threonine}

Figure 39 - Alignment of the signature sequences of several viral $\mathrm{K}^{+}$channels and the different amino acids at position 5. (A) The number in brackets shows the position of the first amino acid of the signature sequence in the entire amino acid sequence of the respective channels. Amino acids in the signature sequence are numbered from 1 to 8 . Position 5 is highlighted in bold letters (aligned with Clustal Omega; Sievers et al., 2011, Madeira et al., 2019). (B) KCVGNLD and KCVMT325 have threonine at position 5 of the signature sequence, compared to the other aligned Kcv channels which possess valine.

Valine and threonine are very similar in shape and volume (fig. 39 B), valine only differs from threonine in the replacement of the hydrophobic methyl group by a hydrophilic hydroxyl group. To see if mutation of threonine to valine has an influence on the channel's single channel gating properties, the mutants KCVGNLD T56V and KCVMT325 T55V were created and examined on the single channel level with the PLB method. Experiments were performed under symmetrical conditions $(100 \mathrm{mM} \mathrm{KCl}, 10 \mathrm{mM}$ HEPES, $\mathrm{pH}$ 7). 
Representative single channel traces for KCVGNLD T56V and KCVMT325 T55V are shown in fig. 40 A. Due to the high open probability and flicker gating at negative voltages, no visible differences can be seen in the single channel traces. Therefore, only traces at positive voltages are shown, from $+40 \mathrm{mV}$ to $+200 \mathrm{mV}$ in $40 \mathrm{mV}$ steps. Compared to the respective single channel traces of the wild type channels (fig. $35 \mathrm{~A}$ ), the obvious reduction of the open probability at higher positive voltages is no longer present in the mutant channels. There is still a visible increase of closed events, but the amount and dwell times of these events seems to be strongly reduced. Mutation from threonine to valine seems to be associated with a loss of the voltage dependency for both channels. Like for the wild type channels, KcVGNLD T56V seems to have a higher single channel conductance than KCVMT325 T55V. After mutation, the above-mentioned different gating that occurs for KCVGNLD and KCVMT325 (fig. 35 B) with episodes of high-conductive open states (GNLD), or short-lasting subconducting states (MT325) are still preserved after mutation of valine to threonine. Fig. $40 \mathrm{~B}$ shows the high-conductive and subconductive states; they are marked with red dotted lines in comparison to the regular open state which is marked with black dotted lines. 


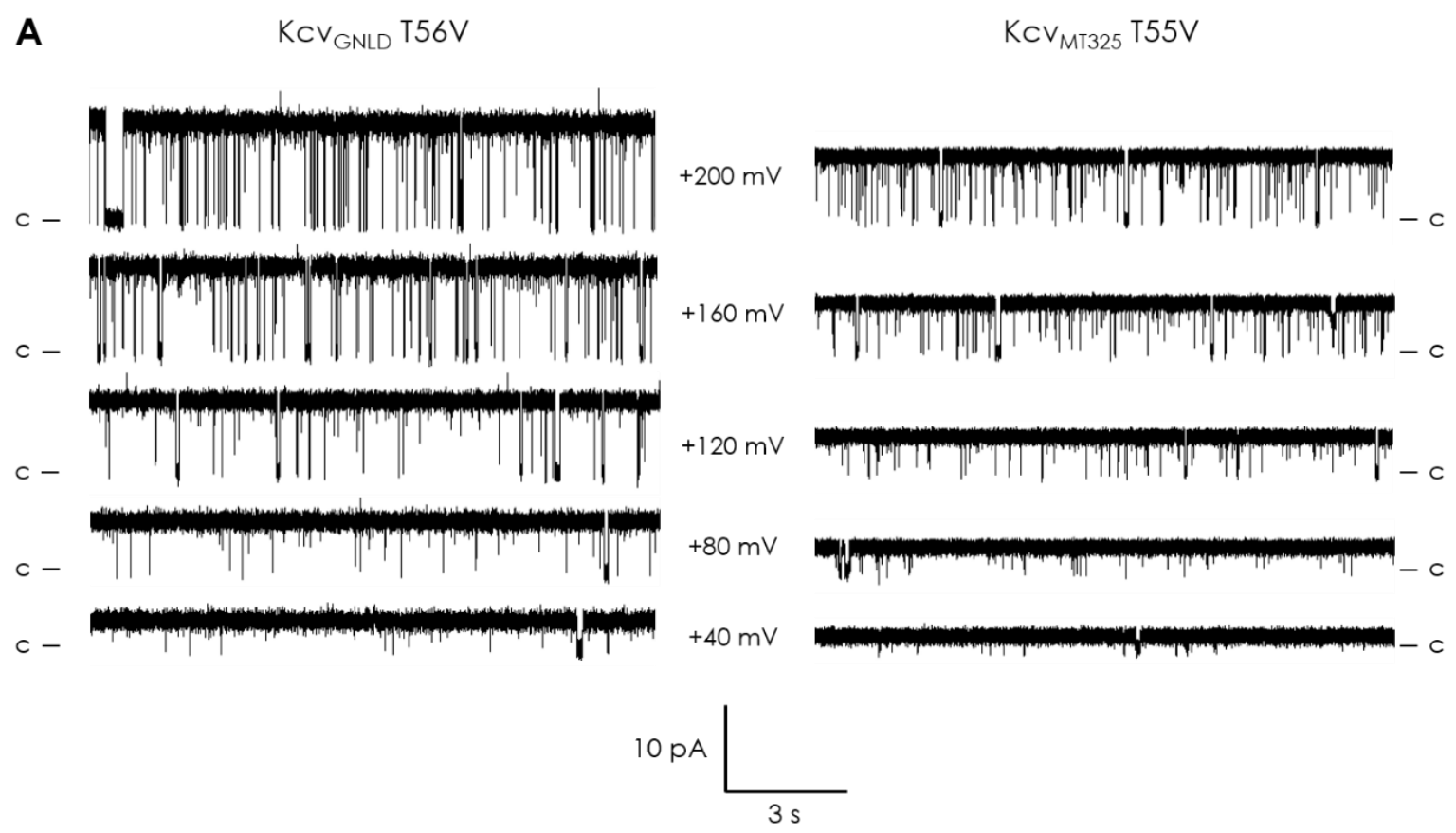

B $\quad \mathrm{KCV}_{\mathrm{GNLD}} \mathrm{T} 56 \mathrm{~V}$

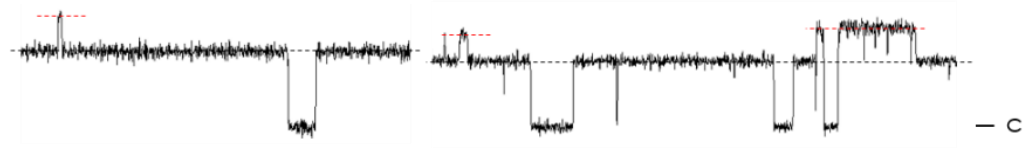

$\mathrm{KCV}_{\mathrm{MT} 325} \mathrm{~T} 55 \mathrm{~V}$

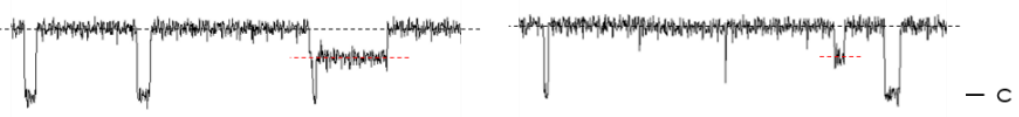

$\frac{10 \mathrm{pA}}{200 \mathrm{~ms}}$

Figure 40 - Representative single channel traces for KcVGNLD T56V and KCVMT325 T55V. (A) Single channel traces of KCVGNLD T56V (left) and KCVMT325 T55V (right), from +200 mV to +40 mV in $40 \mathrm{mV}$ steps. c marks the closed state. (B) Excerpts from various measurements of KCVGNLD T56V (top) and KCVMT325 T55V (bottom) at $+120 \mathrm{mV}$. The main conductivity level is marked with black, while the high (GNLD T56V) and low (MT325 T55V) conductivity levels are marked with red dotted lines. Measurements were performed under symmetrical conditions with $100 \mathrm{mM} \mathrm{KCl}, 10 \mathrm{mM}$ HEPES at $\mathrm{pH}$ 7. For better visualization, data was subsequently filtered with $500 \mathrm{~Hz}$.

The general observations from the representative single channel traces in fig. $40 \mathrm{~A}$ are confirmed by comparing the corresponding i/V relations of KCVGNLD and KCVMT325 to the respective mutants KCVGNLD T56V and $\mathrm{KCV}_{\mathrm{MT} 325} \mathrm{~T} 55 \mathrm{~V}$ (fig. $41 \mathrm{A \& B}$ ). Mutation of valine to threonine directly before the GFG motive in the SF has no effect on the single channel conductance for KCVMT325. For KCVGNLD T56V, there is a small increase of the single channel conductance, for both positive and negative voltages. For better comparison, fig. 
$41 \mathrm{C}$ shows the unitary single channel conductance of KcVGnLd (blue, $45.3 \pm 4.3$ pS), KcVGnLD T56V (blue stripes, $47.6 \pm 4.4 \mathrm{pS}), \mathrm{KCV}_{\mathrm{MT}} 325$ (red, $34.8 \pm 1.4 \mathrm{pS}$ ) and $\mathrm{KcV}_{\mathrm{MT} 325} \mathrm{~T} 55 \mathrm{~V}$ (red stripes, $35.5 \pm 2.3 \mathrm{pS}$ ). Here the small increase in conduction for KCVGNLD T56V over KCVGNLD is again visible while there is no difference between $\mathrm{KCV}_{\text {MT325 }}$ and $\mathrm{KCV}_{\text {MT325 }} \mathrm{T55V}$. It is also clear that $\mathrm{KCV}_{\text {GNLD }}$ and KCVGNLD T56V have a higher single channel conductance than KcVMT325 and KcVMT325 T55V, respectively.

Single channel traces in fig. $40 \mathrm{~A}$ had already indicated that the voltage-dependent decrease of the open probability in the wild type channels KCVGNLD and KCVMT325 is abolished after mutation of valine to threonine at position 55 and 56 of $K_{C V} V_{G N L D}$ and $K_{C V} V_{M T 325}$, respectively. Fig. 41 D and $E$ show the corresponding open probability curves of both wild type channels and their respective mutant channels KCVGNLD T56V and KCVMT325 T55V. Measurements were performed with buffer containing $100 \mathrm{mM} \mathrm{KCl}$. As assumed, the general impressions from the single channel traces are confirmed by the further analyses. For the wild type channels in the voltage range from $-160 \mathrm{mV}$ to $+80 \mathrm{mV}$ the open probability is nearly $100 \%$. For voltages from $+80 \mathrm{mV}$ upwards a steep decrease in open probability to a value below $10 \%$ occurs. The mutant channels KCVGNLD T56V and KCVMT325 T55V lack this voltage dependency. Their open probability is constantly high at nearly $100 \%$, throughout the entire voltage range showing only an insignificant decrease at high positive voltages. Single channel traces had already indicated that the number of closed events for the mutants increases for higher positive voltages. The presumption that the dwell times of these close events is strongly reduced is confirmed. Even though the number of closed events might have increased, the impact on the open probability is so little that it only causes a drop of about 10\% for KCVGNLD T56V and even less than 10\% for KCVMT325 T55V. 
A

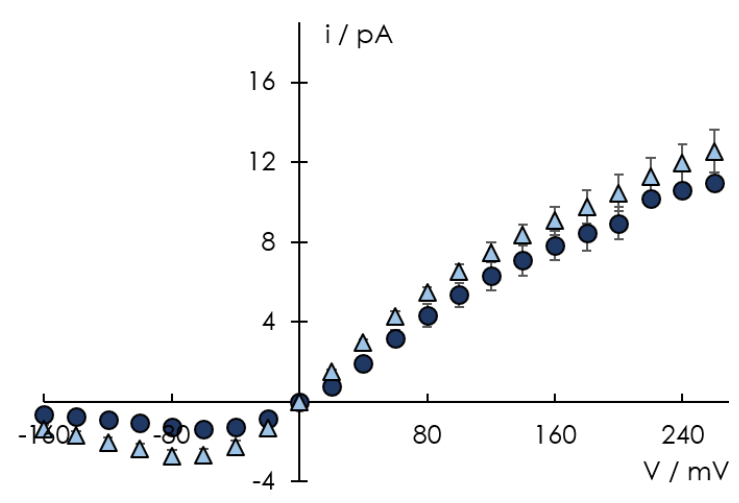

B

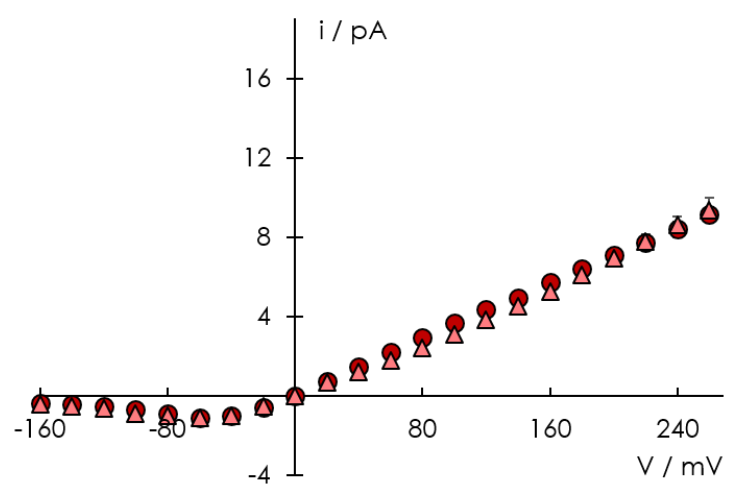

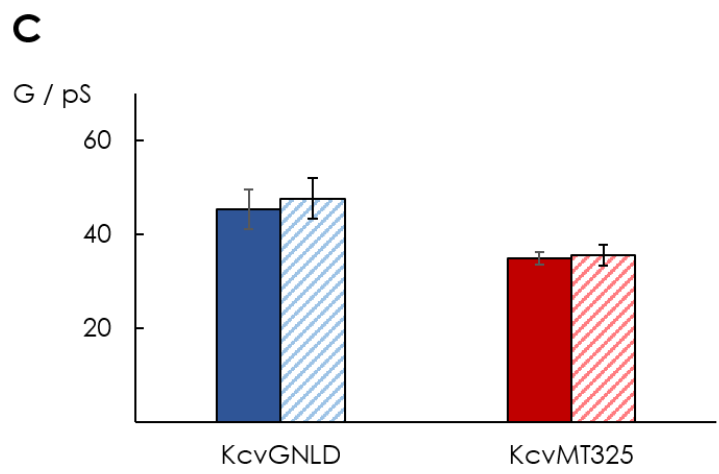

D

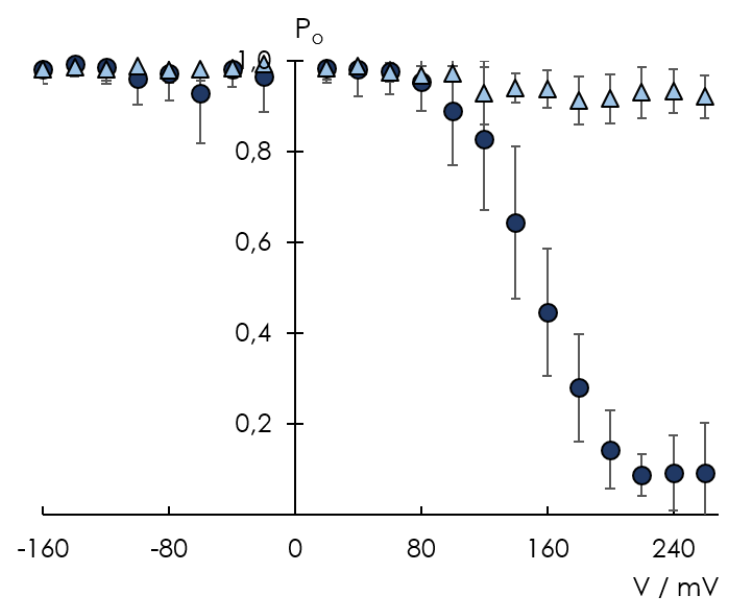

E

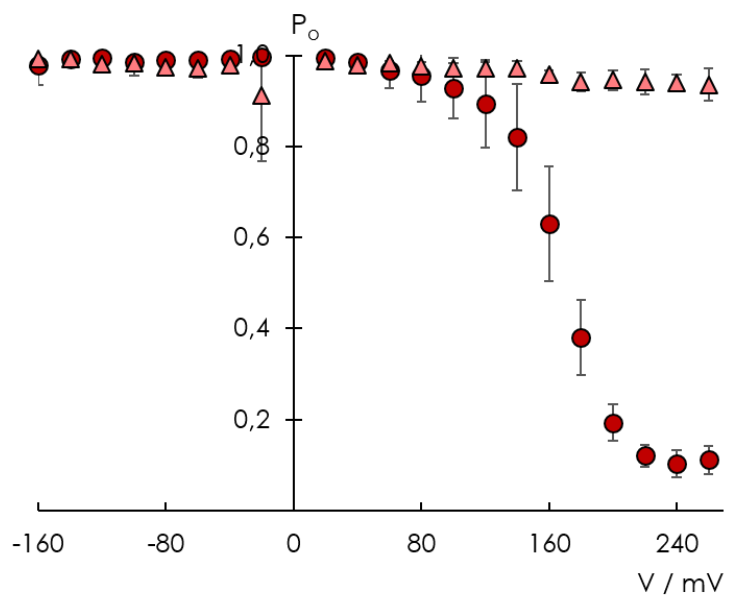

Figure $41-i / V$ curves, single channel conductance and open probability of KcVGNLD T56V and KcVMT325 T55V compared to the wild type channels. i/ $\mathrm{V}$ curves for KCVGNLD T56V (A, blue triangles) and $\mathrm{KCV}_{\text {MT325 }} \mathrm{T5} \mathrm{V} \mathrm{V}$ (B, red triangles) compared to the respective wild type channels KCVGNLD (A, blue cirles) and KCVMT325 (B, red cirles). (C) Single channel conductance of KCVGNLD (blue, $45.3 \pm 4.3$ pS) and KCVMT325 (red, $34.8 \pm 1.4 \mathrm{pS}$ ) compared to KCVGNLD T56V (blue stripes, $47.6 \pm 4.4 \mathrm{pS}$ ) and KCVMT325 T55V (red stripes, $35.5 \pm 2.3 \mathrm{pS}$ ) for positive voltages. Open probability curves for KCVGNLD (D, blue circles) and $\mathrm{KCV}_{\text {MT325 }}$ (E, red circles) compared to $\mathrm{KCV}_{\text {GNLD }} \mathrm{T} 56 \mathrm{~V}$ (D, blue triangles) and $\mathrm{KCV}_{\text {MT325 }}$ (E, red triangles). Data are mean values \pm standard deviation for $\mathrm{n}$ independent measurements

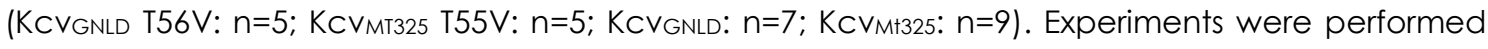
under symmetrical conditions with $100 \mathrm{mM} \mathrm{KCl}$ and $10 \mathrm{mM}$ HEPES at $\mathrm{pH} 7$. 


\subsubsection{Effect of mutation of valine to threonine right before the GFG motive in KCVPBCV-1}

Amino acids in the signature sequence of $\mathrm{K}^{+}$channels are highly conserved. Previous studies have shown that mutation of a single amino acid can lead to a loss of function or to different changes in gating and selectivity properties (Heginbotham et al., 1994; Raja \& Vales, 2009). The previous experiment has shown that mutation of threonine to valine in the viral $\mathrm{K}^{+}$channels KCVGNLD and KCVMT325 on position 5 of the signature sequence (fig. 39 A) alters the channels gating properties. Both wild type channels show a voltage-dependent decrease in the open probability at high positive voltages. After mutation, the voltage dependency in KCVGNLD T56V and KCVMT325 T55V is no longer present. Since valine and threonine are very similar in shape and volume, the exchange of the hydrophobic methyl group in valine to a hydrophilic hydroxyl group must be the cause for the change in gating properties. KCVPBCV-1 is the first $\mathrm{K}^{+}$ channel that has been isolated from a chlorella infecting virus. Even though the amino acid sequence differs partly from KCVGNLD and KCVMT325, there is a high similarity of the amino acids within the signature sequence (fig. 34). At position 5, KCVPBCV-1 has a valine, unlike the threonine in KCVGNLD and KCVMT325. To see if an exchange of valine to threonine also has an effect on the gating properties of KCVPBCV-1, the mutant KCVPBCV-1 V64T was created and examined on the single channel level with the PLB method. Experiments were performed under symmetrical conditions (100 mM KCl, $10 \mathrm{mM} \mathrm{HEPES,} \mathrm{pH} \mathrm{7).}$

Fig. 42 shows representative single channel traces for KCVPBCV-1 and KCVPBCV-1 V64T with two different temporal resolutions. Compared to $\mathrm{KCV}_{\mathrm{GNLD}}$ and $\mathrm{KCV}_{\mathrm{MT} 325}, \mathrm{KCV}_{\mathrm{PBCV}}$-1 has a very low open probability. The open events are characterized by very short dwell times. While at positive voltages the open events are more evenly distributed, at negative voltages the open events occur in bursts, separated by long closed events that can last for several seconds. A higher temporal resolution within these bursts shows that the dwell times for the open events increase with more negative voltages (see chapter 3.2.1). For KCVPBCV-1 V64T the open events are also characterized by short dwell times. The open events are evenly distributed across the entire voltage range, which differs compared to the wild type channel, especially at negative voltages. A higher temporal resolution shows that dwell times within single open events also increase in length with higher negative voltages, as they do for the wild type channel, but long closed events with interrupting bursts are absent in the mutant. A major difference can be seen in the conductance of the channels. At positive voltages $\mathrm{KCV}_{\mathrm{PBCV}-1}$ has a much higher conductance than at negative voltages. Flicker gating also occurs for $\mathrm{KCV}_{\mathrm{PBCV}-1}$, which is why for higher negative voltages the conductance seems to become smaller. The mutation V64T reduces the conductance of the channel strongly, for both negative and positive voltages. For $-120 \mathrm{mV}$ the noise level within the open state is bigger than at $-60 \mathrm{mV}$, indicating that flicker gating still occurs within the mutant $\mathrm{KCV}_{\mathrm{PBCV}-1} \mathrm{~V} 64 \mathrm{~T}$. 


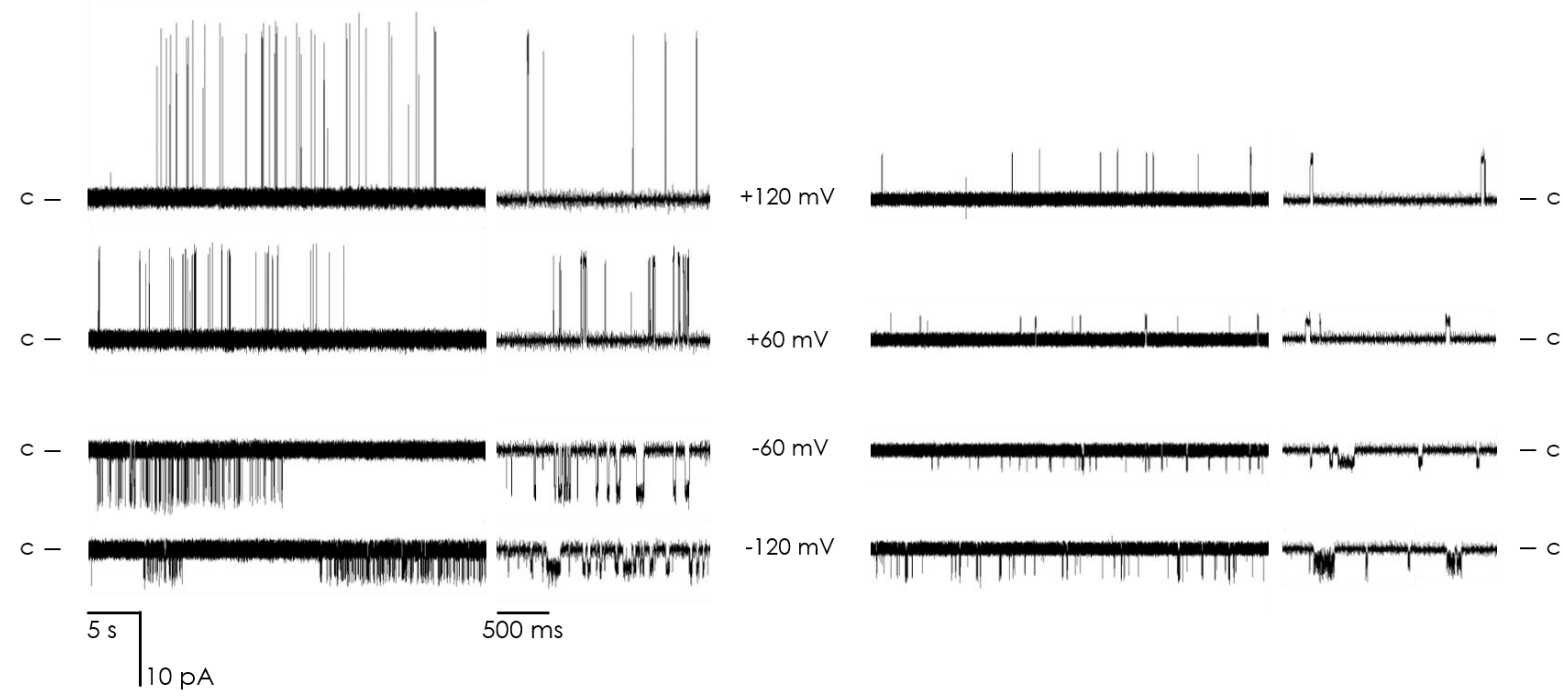

Figure 42 - Representative single channel traces for KCVPBCV-1 and KCVPBCV-1 V64T. Single channel traces of $K_{C V_{P B C V}-1}$ (left) and KCVPBCV-1 V64T (right), from +120 mV to -120 mV in $60 \mathrm{mV}$ steps for two different temporal resolutions. $\mathrm{c}$ marks the closed state of the channels. Measurements were performed under symmetrical conditions with $100 \mathrm{mM} \mathrm{KCl}, 10 \mathrm{mM}$ HEPES at $\mathrm{pH}$ 7. For better visualization, data was subsequently filtered with $500 \mathrm{~Hz}$.

Observations from the single channel traces in fig. 42 are confirmed by the corresponding i/V curve (fig. $43 \mathrm{~A}$ ). The unitary conductance was calculated with equation (1) between 0 and $+160 \mathrm{mV}$. For KCVPBCV-1 it is $174.4 \pm 9.1 \mathrm{pS}$, while for the mutant it is only $50.4 \pm 2.3 \mathrm{pS}$. Both channels show the characteristic negative slope conductance at high negative voltages, due to flicker gating. For KCVPBCV-1 flicker gating starts already at $-80 \mathrm{mV}$, while for $\mathrm{KcV} V 64 \mathrm{~T}$ it starts at about $-90 \mathrm{mV}$. Even though the single channel traces show that the appearance of the open events for $\mathrm{KCV}_{\mathrm{PBCV}-1} \mathrm{~V} 64 \mathrm{~T}$ are slightly different compared to the wild type channel, there is no change in the open probability throughout the measured voltages (fig. 43 B). Both channels still show a slight voltage dependency, characterized by an increase of the open probability with more negative voltages. This voltage dependency can be explained by the increase of dwell times for open events at more negative voltages, shown in fig. 42 in the traces with a higher temporal resolution. 
A

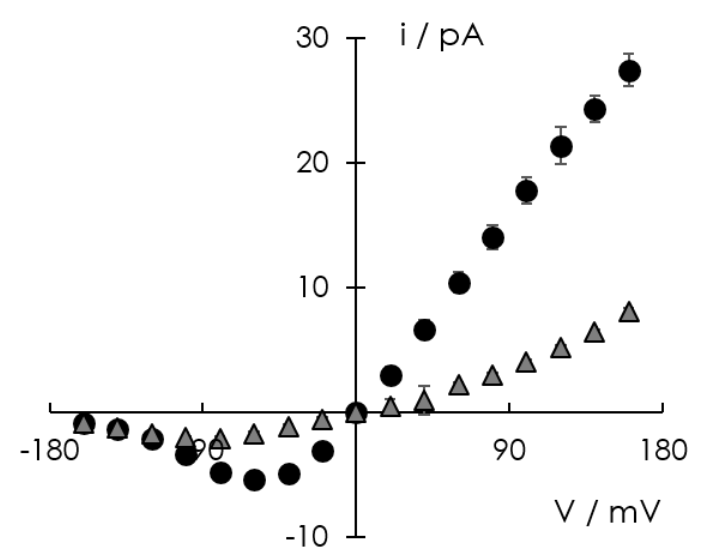

B

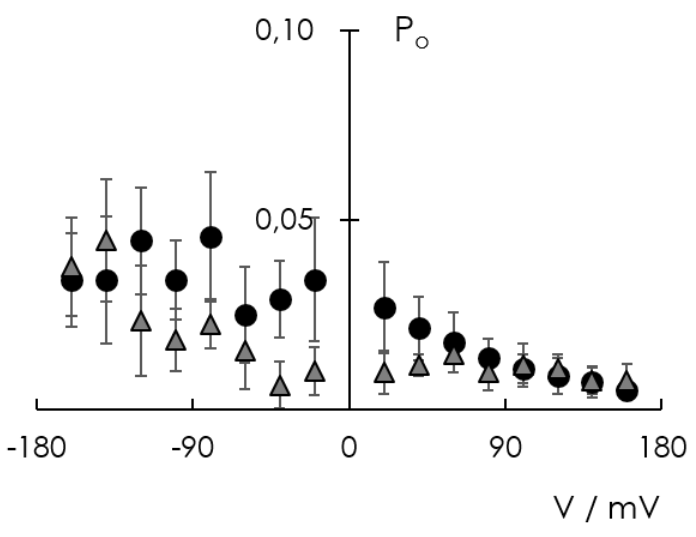

Figure 43 - i/V and open probability curves of KCVPBCV-1 and KCVPBCV-1 V64T. (A) I/V Curves for KCVPBCV-1 (black circles) and $\mathrm{KCV}_{\mathrm{PBCV}-1} \mathrm{~V} 64 \mathrm{~T}$ (grey triangles). (B) Open probability of KCVPBCV-1 (black circles) and $\mathrm{KCVPBCV}_{-1} \mathrm{~V} 64 \mathrm{~T}$ (grey triangles). Data are mean values \pm standard deviation of $\mathrm{n}$ independent measurements (KCVPBCV-1 $\left.\mathrm{n}=8 ; \mathrm{KCVPBCV}_{-1} \mathrm{~V} 64 \mathrm{~T} \mathrm{n}=4\right)$. Measurements were performed under symmetrical conditions with $100 \mathrm{mM} \mathrm{KCl}$ and $10 \mathrm{mM}$ HEPES at $\mathrm{pH} 7$.

\subsubsection{Discussion}

Characterization of KCVGNLD and KCVMT325 have similar single channel properties when measured in PLBS. Both channels have a strong voltage dependency, which manifests as a decrease of the open probability with higher positive voltages. This voltage-dependent decrease shifts towards more positive voltages with increasing $\mathrm{K}^{+}$concentration in the buffer solution. The unitary single channel conductance is slightly higher for KCVGNLD than for $\mathrm{KCV}_{\mathrm{MT} 325}$, which extends throughout different $\mathrm{K}^{+}$concentrations.

An interesting difference in the amino acid sequence of KCVGNLD and KCVMT325 compared to a multitude of aligned $\mathrm{K}^{+}$channels, which possess either valine or isoleucine, or very rarely leucine, (Doyle et al., 1998; MacKinnon, 2003; McCoy \& Nimigean, 2012), is the amino acid threonine at position 5 in the signature sequence (fig. 39). The experiments in this study have shown that mutation of this threonine towards the more common valine (for Kcv channels) within the SF alters the single channel gating of both channels. Mutation of threonine to the more common valine results in a loss of voltage dependency in both channels (fig. 41 D\&E). In addition to the effect on the open probability, the mutation slightly increases the unitary single conductance for KCVGNLD T56V. KCVPBCV-1 on the other hand possesses a valine at position 5 of the signature sequence. Mutation from valine to threonine also alters the channels gating properties, but in a different way than for $\mathrm{KCV}_{G N L D}$ and $\mathrm{KCV}_{\text {MT325. The }}$. The open probability of KCVPBCV-1 V64T stays unaffected, it remains slightly voltage-dependent. The changes in gating, visible in the single channel traces (fig. 42), leave the open probability unaltered. A big difference can be seen in the unitary conductance, which is reduced by over $70 \%$ for $\mathrm{KCV}_{\mathrm{PBCV}-1} \mathrm{~V} 64 \mathrm{~T}$ compared to the wild type channel. Valine and threonine are very similar in size and volume. Nevertheless, an exchange of the hydrophobic methyl group in valine to a hydrophilic hydroxyl group in threonine has a major impact on gating properties of 
these small viral $\mathrm{K}^{+}$channels. Selectivity of $\mathrm{K}^{+}$channels is provided by instant mimicking of the hydration shell of $\mathrm{K}^{+}$. For this, the carbonyl oxygens of the amino acids in the SF are facing towards the pore, providing five oxygen rings, the middle forming exactly the diameter of a single $\mathrm{K}^{+}$ion, creating four $\mathrm{K}^{+}$ binding sites (McCoy \& Nimigean, 2012). Changes within the SF and resulting changes in gating depend on a network of connections between the surrounding amino acids. The amino acid at position 5 is directly involved in the construction of one of these binding sites and thus plays an important role. The hydroxyl group of threonine could form hydrogen bonds with surrounding amino acids which are essential for maintenance of the voltage dependency of KCVGNLD and KCVMT325. Mutation from threonine to valine would eliminate the possibility to form these hydrogen bridges. In KcV on the other hand, mutation from valine to threonine could enable formation of new hydrogen bonds. The smallest change within the SF would lead to an energetically unfavorable conformation, raising the energy barrier for $\mathrm{K}^{+}$ions to pass through the filter. Consequently, the channel would conduct less $\mathrm{K}^{+}$ions, resulting in a smaller single channel conductance.

\subsubsection{Conclusion}

The experiments with small viral $\mathrm{K}^{+}$channels $\mathrm{KCV}_{\mathrm{PBCV}-1}, \mathrm{KCV}_{\mathrm{GNLD}}$ and $\mathrm{KCV}_{\mathrm{MT} 325}$ have shown that mutation of single amino acids can lead to severe changes in the gating of the channels. For example, a single mutation in KCVGNLD and KCVMT325 results in a channel which is virtually $100 \%$ opened throughout the entire voltage range. In $\mathrm{KCV}_{\mathrm{PBCV}-1}$, single mutations can also have a grave impact on either the unitary single channel conductance or the open probability (chapter 3.2.2 \& chapter 3.3.2). This feature makes viral $\mathrm{K}^{+}$ channels particularly suitable pore modules for the construction of synthetic channels. 


\section{List of references}

Abenavoli, A., Difrancesco, M. L., Schroeder, I., Epimashko, S., Gazzarrini, S., Hansen, U. P., Thiel, G., \& Moroni, A. (2009). Fast and slow gating are inherent properties of the pore module of the K+ channel Kcv. Journal of General Physiology, 134 (3), 219-229. https://doi.org/10.1085/jgp.200910266

Accardi, A., Kolmakova-Partensky, L., Williams, C., \& Miller, C. (2004). Ionic Currents Mediated by a Prokaryotic Homologue of CLC Cl- Channels. Journal of General Physiology, 123(2), $109-119$. https://doi.org/10.1085/jgp.200308935

Adam, G., Läuger, P., \& Stark, G. (2009). Physikalische Chemie und Biophysik. https://doi.org/10.1007/9783-642-00424-7

Aggarwal, S. K., \& MacKinnon, R. (1996). Contribution of the S4 segment to gating charge in the Shaker K+ channel. Neuron, 16(6), 1169-1177. https://doi.org/10.1016/S0896-6273(00)80143-9

Alberio, L., Locarno, A., Saponaro, A., Romano, E., Bercier, V., Albadri, S., Simeoni, F., Moleri, S., Pelucchi, S., Porro, A., Marcello, E., Barsotti, N., Kukovetz, K., Boender, A. J., Contestabile, A., Luo, S., Moutal, A., Ji, Y., Romani, G., ... Moroni, A. (2018). A light-gated potassium channel for sustained neuronal inhibition. Nature Methods, 15(1 1), 969-976. https://doi.org/10.1038/s41592-018-0186-9

Alberts, B., Bray, D., Hopkin, K., Johnson, A., Lewis, J., Raff, M., Roberts, K., \& Walter, P. (2009). Essential Cell Biology, third edition. In Garland Science. https://doi.org/citeulike-article-id:4505949

Andersen, O. S., Green, W. N., \& Urban, B. W. (1986). Ion Conduction Through Sodium Channels in Planar Lipid Bilayers. Springer, Boston, MA, USA. https://doi.org/10.1007/978-1-4757-1361-9_15

Armstrong, C. M., \& Bezanilla, F. (1974). Charge movement associated with the opening and closing of the activation gates of the na channels. Journal of General Physiology, 63(5), 533-552. https://doi.org/10.1085/jgp.63.5.533

Arrigoni, C., Schroeder, I., Romani, G., Etten, V., Thiel, G., \& Moroni, A. (2013). The voltage-sensing domain of a phosphatase gates the pore of a potassium channel. Journal of General Physiology, 141 (3), 389-395. https://doi.org/10.1085/jgp.201210940

Ashcroft, F. M. (1999). Ion Channels and Disease. In Academic Press. https://doi.org/10.1177/0022343304040054

Ashcroft, F. M. (2006). From molecule to malady. Nature, 440, $440-447$. https://doi.org/10.1038/nature04707

Bagriantsev, S. N., \& Minor, D. L. (2013). Using Yeast to Study Potassium Channel Function and Interactions with Small Molecules. In M. R. Banghart (Ed.), Chemical Neurobiology: Methods and Protocols (pp. 31-42). Humana Press. https://doi.org/10.1007/978-1-62703-345-9_3

Banerjee, S., \& Nimigean, C. M. (2011). Non-vesicular transfer of membrane proteins from nanoparticles to lipid bilayers. Journal of General Physiology, 137(2), 217-223. https://doi.org/10.1085/jgp.201010558

Baumeister, D. (2010). Einfluss der Transmembrandomänen auf die Funktion und Sortierung von Kaliumkanälen. 1-130.

Ben-Abu, Y., Zhou, Y., Zilberberg, N., \& Yifrach, O. (2009). Inverse coupling in leak and voltage-activated $\mathrm{K}+$ channel gates underlies distinct roles in electrical signaling. Nature Structural and Molecular Biology, 16(1), 71-79. https://doi.org/10.1038/nsmb.1525

Bill, R. M., Henderson, P. J. F., Iwata, S., Kunji, E. R. S., Michel, H., Neutze, R., Newstead, S., Poolman, B., Tate, C. G., \& Vogel, H. (2011). Overcoming barriers to membrane protein structure determination. Nature Biotechnology, 29(4), 335-340. https://doi.org/10.1038/nbt.1833

Bolanos-Garcia, V. M., \& Davies, O. R. (2006). Structural analysis and classification of native proteins from E. coli commonly co-purified by immobilised metal affinity chromatography. Biochimica ef Biophysica Acta - $\quad$ General Subjects, 1760(9), 1304-1313. https://doi.org/10.1016/j.bbagen.2006.03.027 
Braun, C. J., Lachnit, C., Becker, P., Henkes, L. M., Arrigoni, C., Kast, S. M., Moroni, A., Thiel, G., \& Schroeder, I. (2014). Viral potassium channels as a robust model system for studies of membrane-protein interaction. Biochimica et Biophysica Acta - Biomembranes, 1838(4), 1096-1103. https://doi.org/10.1016/j.bbamem.2013.06.010

Buckingham, S. D., Kidd, J. F., Law, R. J., Franks, C. J., \& Sattelle, D. B. (2005). Structure and function of twopore-domain K+ channels: Contributions from genetic model organisms. Trends in Pharmacological Sciences, 26(7), 361-367. https://doi.org/10.1016/j.tips.2005.05.003

Burn, P. (1988). Talking point Amphitropic proteins: a new class of membrane proteins. Trends in Biochemical Sciences, 13(3), 79-83. https://doi.org/10.1016/0968-0004(88)90043-6

Chatelain, F. C., Gazzarrini, S., Fujiwara, Y., Arrigoni, C., Domigan, C., Ferrara, G., Pantoja, C., Thiel, G., Moroni, A., \& Minor, D. L. (2009). Selection of inhibitor-resistant viral potassium channels identifies a selectivity filter site that affects barium and amantadine block. PLOS ONE, 4(10). https://doi.org/10.1371/journal.pone.0007496

Cheley, S., Braha, O., Lu, X., Conlan, S., \& Bayley, H. (1999). A functional protein pore with a "retro" transmembrane domain. Protein Science, 8(6), 1257-1267. https://doi.org/10.1110/ps.8.6.1257

Chen, C. C., England, S., Akopian, A. N., \& Wood, J. N. (1998). A sensory neuron-specific, proton-gated ion channel. Proceedings of the National Academy of Sciences of the United States of America, 95(17), 10240-10245. https://doi.org/10.1073/pnas.95.17.10240

Chen, R. (2012). Bacterial expression systems for recombinant protein production: E. coli and beyond. Biotechnology Advances, 30(5), 1102-1 107. https://doi.org/10.1016/j.biotechadv.201 1.09.013

Choe, S. (2002). Potassium Channel Structures. Nature Reviews Neuroscience, 3(2), $115-121$. https://doi.org/10.1038/nrn727

Choi, K. L., Aldrich, R. W., \& Yellen, G. (1991). Tetraethylammonium blockade distinguishes two inactivation mechanisms in voltage-activated K+ channels. Proceedings of the National Academy of Sciences of the United States of America, 88(12), 5092-5095. https://doi.org/10.1073/pnas.88.12.5092

Christie, J. M. (2007). Phototropin Blue-Light Receptors. Annual Review of Plant Biology, 58(1), 21-45. https://doi.org/10.1 146/annurev.arplant.58.032806.103951

Clapham, D. E. (1998). At last, the structure of an ion-selective channel. Nature Publishing, 5(5), $342-344$. https://doi.org/10.1038/nsb0598-342

Cordero-Morales, J. F., Cuello, L. G., Zhao, Y., Jogini, V., Cortes, D. M., Roux, B., \& Perozo, E. (2006). Molecular determinants of gating at the potassium-channel selectivity filter. Nature Structural and Molecular Biology, 13(4), 31 1-318. https://doi.org/10.1038/nsmb1069

Cosentino, C., Alberio, L., Gazzarrini, S., Aquila, M., Romano, E., Cermenati, S., Zuccolini, P., Petersen, J., Beltrame, M., Van Etten, J. L., Christie, J. M., Thiel, G., \& Moroni, A. (2014). Engineering of a light-gated potassium channel. Science, 9844 (2011), 707-710. https://doi.org/10.1126/science.aaa2787

Cregg, J. M., Cereghino, J. L., Shi, J., \& Higgins, D. R. (2000). Recombinant protein expression in Pichia pastoris. Applied Biochemistry and Biotechnology - Part B Molecular Biotechnology, 16(1), $23-52$. https://doi.org/10.1385/MB:16:1:23

Cuello, L. G., Jogini, V., Cortes, D. M., \& Perozo, E. (2010). Structural mechanism of C-type inactivation in K+ channels. Nature, 466(7303), 203-208. https://doi.org/10.1038/nature09153

Cuello, L. G., Romero, J. G., Cortes, D. M., \& Perozo, E. (1998). pH-Dependent Gating in the Streptomyces li V idans K + Channel. Biochemistry, 37(10), 3229-3236. https://doi.org/10.1021/bi972997x

De Schutter, K., Lin, Y. C., Tiels, P., Van Hecke, A., Glinka, S., Weber-Lehmann, J., Rouzé, P., Van De Peer, Y., \& Callewaert, N. (2009). Genome sequence of the recombinant protein production host Pichia pastoris. Nature Biotechnology, 27(6), 561-566. https://doi.org/10.1038/nbt.1544

De Vos, W. M. (1999). Gene expression systems for lactic acid bacteria. Current Opinion in Microbiology, 2(3), 289-295. https://doi.org/10.1016/S1369-5274(99)80050-2

Deamer, D. W., \& Bramhall, J. (1986). Permeability of lipid bilayers to water and ionic solutes. Chemistry 
and Physics of Lipids, 40(2-4), 167-188. https://doi.org/10.1016/0009-3084(86)90069-1

Dörr, J. M., Koorengevel, M. C., Schäfer, M., Prokofyev, A. V., Scheidelaar, S., Van Der Cruijsen, E. A. W., Dafforn, T. R., Baldus, M., \& Killian, J. A. (2014). Detergent-free isolation, characterization, and functional reconstitution of a tetrameric K+ channel: The power of native nanodiscs. Proceedings of the National Academy of Sciences of the United States of America, 1111(52), 18607-18612. https://doi.org/10.1073/pnas.1416205112

Doyle, D. A., Cabral, J. M., Pfuetzner, R. A., Kuo, A., Gulbis, J. M., Cohen, S. L., Chait, B. T., \& MacKinnon, R. (1998). The structure of the potassium channel: molecular basis of $K+$ conduction and selectivity. Science (New York, N.Y.), 280 (5360), 69-77. https://doi.org/10.1126/science.280.5360.69

Edgcomb, S. P., \& Murphy, K. P. (2002). Variability in the pKa of histidine side-chains correlates with burial within proteins. Proteins: Structure, Function and Genetics, 49(1), 1-6. https://doi.org/10.1002/prot.10177

Fischer, W. B., \& Sansom, M. S. P. (2002). Viral ion channels: Structure and function. Biochimica et Biophysica Acta - Biomembranes, 1561 (1), 27-45. https://doi.org/10.1016/S0304-4157(01)00009-0

Fitzgerald, L. A., Graves, M. V., Li, X., Hartigan, J., Pfitzner, A. J. P., Hoffart, E., \& Van Etten, J. L. (2007). Sequence and annotation of the $288-\mathrm{kb}$ ATCV-1 virus that infects an endosymbiotic chlorella strain of the heliozoon Acanthocystis turfacea. Virology, 362(2), 350-361. https://doi.org/10.1016/j.virol.2006.12.028

Focke, P. J., Hein, C., Hoffmann, B., Matulef, K., Bernhard, F., Dötsch, V., \& Valiyaveetil, F. I. (2016). Combining in Vitro Folding with Cell Free Protein Synthesis for Membrane Protein Expression. Biochemistry, 55(30), 4212-4219. https://doi.org/10.1021/acs.biochem.6b00488

Gazzarrini, S., Kang, M., Abenavoli, A., Romani, G., Olivari, C., Gaslini, D., Ferrara, G., Van Etten, J. L., Kreim, M., Kast, S. M., Thiel, G., \& Moroni, A. (2009). Chlorella virus ATCV-1 encodes a functional potassium channel of 82 amino acids. The Biochemical Journal, 420(2), $295-303$. https://doi.org/10.1042/BJ20090095

Gazzarrini, S., Kang, M., Epimashko, S., Van Etten, J. L., Dainty, J., Thiel, G., \& Moroni, A. (2006). Chlorella virus MT325 encodes water and potassium channels that interact synergistically. Proceedings of the National Academy of Sciences of the United States of America, 103(14), 5355-5360. https://doi.org/10.1073/pnas.0600848103

Gazzarrini, S., Severino, M., Lombardi, M., Morandi, M., Difrancesco, D., Van Etten, J. L., Thiel, G., \& Moroni, A. (2003). The viral potassium channel Kcv: Structural and functional features. FEBS Letters, $552(1)$, 12-16. https://doi.org/10.1016/S0014-5793(03)00777-4

Gazzarrini, S., Van Etten, J. L., Difrancesco, D., Thiel, G., \& Moroni, A. (2002). Voltage-dependence of virusencoded miniature $\mathrm{K}+$ channel KcV. Journal of Membrane Biology, 187(1), 15-25. https://doi.org/10.1007/s00232-001-0147-5

Gebhardt, M. (2010). Structure / Function Analysis of the Viral Potassium Channel Kcv. PhD Thesis, Technische Universität Darmstadt.

Gebhardt, M., Henkes, L. M., Tayefeh, S., Hertel, B., Greiner, T., Van Etten, J. L., Baumeister, D., Cosentino, C., Moroni, A., Kast, S. M., \& Thiel, G. (2012). Relevance of lysine snorkeling in the outer transmembrane domain of small viral potassium ion channels. Biochemistry, 51 (28), 5571-5579. https://doi.org/10.1021/bi3006016

Gerstein, M., \& Hegyi, H. (1998). Comparing genomes in terms of protein structure: Surveys of a finite parts list. FEMS Microbiology Reviews, 22 (4), 277-304. https://doi.org/10.1016/S0168-6445(98)00019-9

Gonzalez, M. E., \& Carrasco, L. (2003). Viroporins. FEBS Letters, 552(1), 28-34. https://doi.org/10.1016/S00145793(03)00780-4

Gordon, E., Horsefield, R., Swarts, H. G. P., de Pont, J. J. H. H. M., Neutze, R., \& Snijder, A. (2008). Effective high-throughput overproduction of membrane proteins in Escherichia coli. Protein Expression and Purification, 62(1), 1-8. https://doi.org/10.1016/j.pep.2008.07.005

Greiner, T. (201 1). Characterization of novel potassium transport proteins from Chlorella viruses. PhD Thesis, 
Technische Universität Darmstadt, 1-115. http://tuprints.ulb.tu-darmstadt.de/2685/

Hagn, F., Etzkorn, M., Raschle, T., \& Wagner, G. (2013). Optimized phospholipid bilayer nanodiscs facilitate high-resolution structure determination of membrane proteins. Journal of the American Chemical Society, 135(5), 1919-1925. https://doi.org/10.1021/ja310901f

Hamacher, K., Greiner, T., Ogata, H., van Etten, J. L., Gebhardt, M., Villarreal, L. P., Cosentino, C., Moroni, A., \& Thiel, G. (2012). Phycodnavirus potassium ion channel proteins question the virus molecular piracy hypothesis. PLOS ONE, 7(6), 1-9. https://doi.org/10.1371/journal.pone.0038826

Hanke, W., \& Schlue, W. R. (1993). Planar lipid bilayers: methods and applications. Academic Press.

Hansen, A. L., \& Kay, L. E. (2014). Measurement of histidine pKa values and tautomer populations in invisible protein states. Proceedings of the National Academy of Sciences of the United States of America, 111 (17). https://doi.org/10.1073/pnas.1400577111

Hartel, A. J. W., Ong, P., Schroeder, I., Hunter Giese, M., Shekar, S., Clarke, O. B., Zalk, R., Marks, A. R., Hendrickson, W. A., \& Shepard, K. L. (2018). Single-channel recordings of RyRl at microsecond resolution in CMOS-suspended membranes. Proceedings of the National Academy of Sciences of the United States of America, 115(8), E1789-E1798. https://doi.org/10.1073/pnas.1712313115

Heginbotham, L., Lu, Z., Abramson, T., \& MacKinnon, R. (1994). Mutations in the K+ channel signature sequence. Biophysical Journal, 66(4), 1061-1067. https://doi.org/10.1016/S0006-3495(94)80887-2

Heimburg, T. (2010). Lipid ion channels. Biophysical Chemistry, 150(1-3), 2-22. https://doi.org/10.1016/j.bpc.2010.02.018

Hertel, B., Tayefeh, S., Kloss, T., Hewing, J., Gebhardt, M., Baumeister, D., Moroni, A., Thiel, G., \& Kast, S. M. (2010). Salt bridges in the miniature viral channel Kcv are important for function. European Biophysics Journal, 39(7), 1057-1068. https://doi.org/10.1007/s00249-009-0451-z

Hille, B. (2001). Ion Channels of Excitable Membranes. Third edition. In Sunderland Massachusetts USA. Sinaver Associates Inc. https://doi.org/10.1007/3-540-29623-9_5640

Hirano-Iwata, A., Ishinari, Y., Yamamoto, H., \& Niwano, M. (2015). Micro- and nano-technologies for lipid bilayer-based ion-channel functional assays. Chemistry - An Asian Journal, 10(6), 1266-1274. https://doi.org/10.1002/asia.201403391

Hirano-Iwata, A., Ishinari, Y., Yoshida, M., Araki, S., Tadaki, D., Miyata, R., Ishibashi, K., Yamamoto, H., Kimura, Y., \& Niwano, M. (2016). Reconstitution of Human Ion Channels into Solvent-free Lipid Bilayers Enhanced by Centrifugal Forces. Biophysical Journal, 110(10), 2207-2215. https://doi.org/10.1016/j.bpj.2016.04.010

Hodgkin, A. L., \& Huxley, A. F. (1939). Action Potentials Recorded from Inside a Nerve Fibre. Nature, 144 (6), 710-711. https://doi.org/10.1038/144710a0

Hodgkin, A. L., Huxley, A. F., \& Katz, B. (1952). Measurement Of Curren-Voltage Relations In The Membrane Of The Giant Axon Of Loligo. The Journal of Physiology, 116, 424-448. https://doi.org/10.1113/jphysiol.1952.sp004716

Hoffgaard, F., Kast, S. M., Moroni, A., Thiel, G., \& Hamacher, K. (2015). Tectonics of a K+ channel: The importance of the N-terminus for channel gating. Biochimica et Biophysica Acta-Biomembranes, 1848(12), 3197-3204. https://doi.org/10.1016/j.bbamem.2015.09.015

Hoshi, T., \& Armstrong, C. M. (2013). C-type inactivation of voltage-gated K+ channels: Pore constriction or dilation? Journal of General Physiology, 141 (2), 151-160. https://doi.org/10.1085/jgp.201210888

Hoshi, T., Zagotta, W. N., \& Aldrich, R. W. (1990). Shaker Potassium Channel Inactivation. Science, 250(October), 553-558.

Hoshi, T., Zagotta, W. N., \& Aldrich, R. W. (1991). Two types of inactivation in Shaker K+ channels: Effects of alterations in the carboxy-terminal region. Neuron, 7(4), 547-556. https://doi.org/10.1016/08966273(91)90367-9

Hurley, J. H. (2003). Membrane Proteins: Adapting to Life at the Interface. Chemistry \& Biology, 10(1), 2-3. https://doi.org/10.1016/PII 
Ide, M., Maeda, Y., \& Kitano, H. (1997). Effect of hydrophobicity of amino acids on the structure of water. Journal of Physical Chemistry B, 101 (35), 7022-7026. https://doi.org/10.1021/jp971334m

Imai, S., Osawa, M., Takeuchi, K., \& Shimada, I. (2010). Structural basis underlying the dual gate properties of KcsA. Proceedings of the National Academy of Sciences of the United States of America, 107(14), 6216-6221. https://doi.org/10.1073/pnas.0911270107

Jensen, M., Jogini, V., Borhani, D. W., Leffler, A. E., Dror, R. O., \& Shaw, D. E. (2012). Mechanism of voltage gating in potassium channels. Science, 336 (6078), 229-233. https://doi.org/10.1 126/science.1216533

Jiang, Y., Lee, A., Chen, J., Cadene, M., Chait, B. T., \& MacKinnon, R. (2002). The open pore conformation of potassium channels. Nature, 417 (6888), 523-546. https://doi.org/10.1038/417523a

Jiang, Y., Lee, A., Chen, J., Ruta, V., Cadene, M., Chait, B. T., \& MacKinnon, R. (2003). X-ray structure of a voltage-dependent K+ channel. Nature, 423(6935), 33-41. https://doi.org/10.1038/nature01580

Katakuse, I., Matsuo, T., Wollnik, H., \& Matsuda, H. (1979). Multiply charged ions in field desorption mass spectra of peptides containing basic amino acids. Organic Mass Spectrometry, 14(8), 457-458. https://doi.org/10.1002/oms.1210140813

Katzen, F., Chang, G., \& Kudlicki, W. (2005). The past, present and future of cell-free protein synthesis. Trends in Biotechnology, 23(3), 150-156. https://doi.org/10.1016/j.tibtech.2005.01.003

Khan, M. S., Dosoky, N. S., Berdiev, B. K., \& Williams, J. D. (2016). Electrochemical impedance spectroscopy for black lipid membranes fused with channel protein supported on solid-state nanopore. European Biophysics Journal, 45 (8), 843-852. https://doi.org/10.1007/s00249-016-1156-8

Kingsman, S. M., Kingsman, A. J., \& Mellor, J. (1987). The production of mammalian proteins in Saccharomyces cerevisiae. Trends in Biotechnology, 5(2), 53-57. https://doi.org/10.1016/0167$7799(87) 90038-2$

Kiss, L., LoTurco, J., \& Korn, S. J. (1999). Contribution of the selectivity filter to inactivation in potassium channels. Biophysical Journal, 76(1 I), 253-263. https://doi.org/10.1016/S0006-3495(99)77194-8

Klammt, C., Löhr, F., Schäfer, B., Haase, W., Dötsch, V., Rüterjans, H., Glaubitz, C., \& Bernhard, F. (2004). High level cell-free expression and specific labeling of integral membrane proteins. European Journal of Biochemistry, 271 (3), 568-580. https://doi.org/10.1111/j.1432-1033.2003.03959.x

Konigsberg, W. (1972). Reduction of Disulfide Bonds in Proteins with Dithiothreitol. Methods in Enzymology, 25(C), 185-188. https://doi.org/10.1016/S0076-6879(72)25015-7

Krämer, U., Cotter-Howells, J. D., Charnock, J. M., Baker, A. J. M., \& Smith, J. A. C. (1996). Free histidine as a metal chelator in plants that accumulate nickel. In Nature (Vol. 379, Issue 6566, pp. 635-638). https://doi.org/10.1038/379635a0

Krol, E., \& Trebacz, K. (2000). Ways of ion channel gating in plant cells. Annals of Botany, 86(3), $449-469$. https://doi.org/10.1006/anbo.2000.1226

Kuang, Q., Purhonen, P., \& Hebert, H. (2015). Structure of potassium channels. Cellular and Molecular Life Sciences, 72(19), 3677-3693. https://doi.org/10.1007/s00018-015-1948-5

Kunji, E. R. S., Slotboom, D. J., \& Poolman, B. (2003). Lactococcus lactis as host for overproduction of functional membrane proteins. Biochimica et Biophysica Acta - Biomembranes, 1610(1), 97-108. https://doi.org/10.1016/S0005-2736(02)00712-5

Kurata, H. T., \& Fedida, D. (2006). A structural interpretation of voltage-gated potassium channel inactivation. Progress in Biophysics and Molecular Biology, 92(2), 185-208. https://doi.org/10.1016/j.pbiomolbio.2005.10.001

Kyte, J., \& Doolittle, R. F. (1982). A simple method for displaying the hydropathic character of a protein. Journal of Molecular Biology, 157(1), 105-132. https://doi.org/10.1016/0022-2836(82)90515-0

Labro, A. J., \& Snyders, D. J. (2012). Being flexible: The voltage-controllable activation gate of Kv channels. Frontiers in Pharmacology, 3 SEP(September), 1-12. https://doi.org/10.3389/fphar.2012.00168

Läuger, P. (1985). Mechanisms of Biological Ion Transport - Carriers, Channels, and Pumps in Artificial Lipid 
Membranes. Angewandte Chemie International Edition in English, 24(11), 905-923. https://doi.org/10.1002/anie.198509051

Li, L., Vorobyov, I., MacKerell, A. D., \& Allen, T. W. (2008). Is arginine charged in a membrane? Biophysical Journal, 94 (2), L1 1-L13. https://doi.org/10.1529/biophysj.107.121566

Lockless, S. W., Zhou, M., \& MacKinnon, R. (2007). Structural and thermodynamic properties of selective ion binding in a K+ channel. PLoS Biology, 5(5), 1079-1088. https://doi.org/10.1371/journal.pbio.0050121

Lodish, H. (2003). Molecular Cell Biology. 29, 973. https://doi.org/10.1016/S1470-8175(01)00023-6

Loewenthal, R., Sancho, J., \& Fersht, A. R. (1992). Histidine-aromatic interactions in barnase. Elevation of histidine pKa and contribution to protein stability. Journal of Molecular Biology, 224 (3), 759-770. https://doi.org/10.1016/0022-2836(92)90560-7

Long, S. B., Campbell, E. B., \& MacKinnon, R. (2005). Crystal Structure of a Mammalian Voltage-Dependent Shaker Family Kp Channel. Science, 309(5736), 897-903. https://doi.org/10.1016/j.jssc.2005.07.007

López-Barneo, J., Hoshi, T., Heinemann, S. H., \& Aldrich, R. W. (1993). Effects of external cations and mutations in the pore region on C-type inactivation of Shaker potassium channels. Receptors \& Channels, 1(1), 61-71.

Lummis, S. C. R., Beene, D. L., Lee, L. W., Lester, H. A., Broadhurst, R. W., \& Dougherty, D. A. (2005). Cis-trans isomerization at a proline opens the pore of a neurotransmitter-gated ion channel. Nature, 438(7065), 248-252. https://doi.org/10.1038/nature04130

Mach, T., Chimerel, C., Fritz, J., Fertig, N., Winterhalter, M., \& Fütterer, C. (2008). Miniaturized planar lipid bilayer: Increased stability, low electric noise and fast fluid perfusion. Analytical and Bioanalytical Chemistry, 390(3), 841-846. https://doi.org/10.1007/s00216-007-1647-7

MacKinnon, R. (2003). Potassium channels. FEBS Letters, 555(1), 62-65. https://doi.org/10.1016/S00145793(03)01 104-9

Madeira, F., Park, Y. M., Lee, J., Buso, N., Gur, T., Madhusoodanan, N., Basutkar, P., Tivey, A. R. N., Potter, S. C., Finn, R. D., \& Lopez, R. (2019). The EMBL-EBI search and sequence analysis tools APIs in 2019. Nucleic Acids Research, 47(W1), W636-W641. https://doi.org/10.1093/nar/gkz268

Marsh, D. (1990). CRC handbook of lipid bilayers. CRC Press. https://books.google.de/books?id=lbbwAAAAMAAJ

Mateo, C., Fernandez-Lorente, G., Pessela, B. C. C., Vian, A., Carrascosa, A. V., Garcia, J. L., FernandezLafuente, R., \& Guisan, J. M. (2001). Affinity chromatography of polyhistidine tagged enzymes - New dextran-coated immobilized metal ion affinity chromatography matrices for prevention of undesired multipoint adsorptions. Journal of Chromatography A, 915(1-2), 97-106. https://doi.org/10.1016/S0021-9673(01)00626-4

Matthew, J. B., Gurd, F. R. N., Garcia-Moreno, B. E., Flanagan, M. A., March, K. L., \& Shire, S. J. (1985). Phdependent processes in protein. Critical Reviews in Biochemistry and Molecular Biology, 18(2), 91197. https://doi.org/10.3109/10409238509085133

McCoy, J. G., \& Nimigean, C. M. (2012). Structural correlates of selectivity and inactivation in potassium channels. Biochimica et Biophysica Acta - Biomembranes, 1818(2), 272-285. https://doi.org/10.1016/j.bbamem.2011.09.007

McGuire, H., \& Blunck, R. (2015). Studying Clustering of KcsA Channels using Single-Channel VoltageClamp Fluorescence Imaging. Biophysical Journal, 108(2), 440a. https://doi.org/10.1016/j.bpj.2014.11.2404

Montal, M., \& Mueller, P. (1972). Formation of bimolecular membranes from lipid monolayers and a study of their electrical properties. Proceedings of the National Academy of Sciences of the United States of America, 69(12), 3561-3566. https://doi.org/10.1073/pnas.69.12.3561

Moroni, A., Viscomi, C., Sangiorgio, V., Pagliuca, C., Meckel, T., Horvath, F., Gazzarrini, S., Valbuzzi, P., Van Etten, J. L., Difrancesco, D., \& Thiel, G. (2002). The short N-terminus is required for functional expression 
of the virus-encoded miniature $\mathrm{K}+$ channel KcV. FEBS Letters, 530(1-3), 65-69. https://doi.org/10.1016/S0014-5793(02)03397-5

Müller, P., Rudin, D. O., Ti Tien, H., \& Wescott, W. C. (1962). Reconstitution of cell membrane structure in vitro and its transformation into an excitable system. Nature, 194(4032), 979-980. https://doi.org/10.1038/194979a0

Needham, M., Gooding, C., Hudson, K., Antoniou, M., Grosveld, F., \& Hollis, M. (1992). LCR/MEL: A versatile system for high-level expression of heterologous proteins in erythroid cells. Nucleic Acids Research, 20(5), 997-1003. https://doi.org/10.1093/nar/20.5.997

Neher, E., \& Sakmann, B. (1976). Noise Analysis Of Drug Induced Voltage Clamp Currents In Denervated Frog Muscle Fibres. Journal of Physiology, 258, 705-729. https://doi.org/10.1113/jphysiol.1976.sp011442

Nelson, N., Anholt, R., Lindstrom, J., \& Montal, M. (1980). Reconstitution of purified acetylholine receptors with functional ion channels in planar lipid bilayers. Proceedings of the National Academy of Sciences of the United States of America, 77(5), 3057-3061. https://doi.org/10.1073/pnas.77.5.3057

Neyton, J., \& Miller, C. (1988a). Discrete Ba2+ block as a probe of ion occupancy and pore structure in the high-conductance Ca2+-activated K+ channel. Journal of General Physiology, 92 (5), 569-586. https://doi.org/10.1085/jgp.92.5.569

Neyton, J., \& Miller, C. (1988b). Potassium blocks barium permeation through a calcium-activated potassium channel. Journal of General Physiology, 92(5), 549-567. https://doi.org/10.1085/jgp.92.5.549

Nozaki, Y., \& Tanford, C. (1967). [84] Examination of titration behavior. Methods in Enzymology, 11 (C), $715-$ 734. https://doi.org/10.1016/S0076-6879(67)11088-4

Ogielska, E. M., Zagotta, W. N., Hoshi, T., Heinemann, S. H., Haab, J., \& Aldrich, R. W. (1995). Cooperative subunit interactions in C-type inactivation of K channels. Biophysical Journal, 69(6), 2449-2457. https://doi.org/10.1016/S0006-3495(95)80114-1

Ohndorf, U. M., \& MacKinnon, R. (2005). Construction of a cyclic nucleotide-gated KcsA K+ channel. Journal of Molecular Biology, 350 (5), 857-865. https://doi.org/10.1016/j.jmb.2005.05.050

Ostermeier, C., \& Michel, H. (1997). Crystallization of membrane proteins. Current Opinion in Structural Biology, 7(5), 697-701. https://doi.org/10.1016/S0959-440X(97)80080-2

Pagliuca, C., Goetze, T. A., Wagner, R., Thiel, G., Moroni, A., \& Parcej, D. (2007). Molecular properties of KCv, a virus encoded K+ channel. Biochemistry, 46 (4), 1079-1090. https://doi.org/10.1021/bi061530w

Panyi, G., Sheng, Z., \& Deutsch, C. (1995). C-type inactivation of a voltage-gated K+ channel occurs by a cooperative mechanism. Biophysical Journal, 69(3), 896-903. https://doi.org/10.1016/S00063495(95)79963-5

Perozo, E., Cortes, D. M., \& Cuello, L. G. (1999). Structural Rearrangements Underlying K+-Channel Activation Gating. Science, 285(5424), 73-78. https://doi.org/10.1126/science.285.5424.73

Perrin, D. D., \& Sharma, V. S. (1967). Histidine Complexes with some Bivalent Cations. Journal of the Chemical Society A: Inorganic, Physical, Theoretical, 0(724), 724-728. https://doi.org/10.1039/J19670000724

Pfeifer, T. A. (1998). Expression of heterologous proteins in stable insect cell culture. Current Opinion in Biotechnology, 9(5), 518-521. https://doi.org/10.1016/S0958-1669(98)80039-6

Piasta, K. N., Theobald, D. L., \& Miller, C. (2011). Potassium-selective block of barium permeation through single KcsA channels. Journal of General Physiology, 138(4), 421-436. https://doi.org/10.1085/jgp.201110684

Plugge, B., Gazzarrini, S., Nelson, M., Cerana, R., Van Etten, J. L., Derst, C., DiFrancesco, D., Moroni, A., \& Thiel, G. (2000). A potassium channel protein encoded by chlorella virus PBCV-1. Science, 287(5458), 1641-1644. https://doi.org/10.1126/science.287.5458.1641

Raja, M., \& Vales, E. (2009). Changing Val-76 towards Kir channels drastically influences the folding and 
gating properties of the bacterial potassium channel KcsA. Biophysical Chemistry, 144(3), 95-100. https://doi.org/10.1016/j.bpc.2009.06.006

Rauh, O. (2018). Molecular explanations for gating in simple model K+ channels. PhD Thesis, Technische Universität Darmstadt.

Rauh, O., Urban, M., Henkes, L. M., Winterstein, T., Greiner, T., Van Etten, J. L., Moroni, A., Kast, S. M., Thiel, G., \& Schroeder, I. (2017). Identification of Intrahelical Bifurcated H-Bonds as a New Type of Gate in $\mathrm{K}+$ Channels [Research-article]. Journal of the American Chemical Society, 139(22), 7494-7503. https://doi.org/10.1021/jacs.7b01158

Rotem, D., Mason, A., \& Bayley, H. (2010). Inactivation of the KcsA potassium channel explored with heterotetramers. Journal of General Physiology, 135(1), 29-42. https://doi.org/10.1085/jgp.200910305

Ruppersberg, J. P., Frank, R., Pongs, O., \& Stocker, O. (1991). Cloned neuronal Ik(A) channels reopen during recovery from inactivation. Nature, 353, 657-660. https://doi.org/10.1038/353657a0

Sakmann, B., Patlak, J., \& Neher, E. (1980). Single acetylcholine-activated channels show burst-kinetics in presence of desensitizing concentrations of agonist. Nature, 286(5768), 71-73. https://doi.org/10.1038/286071a0

Sansom, M. S. P., Shrivastava, I. H., Bright, J. N., Tate, J., Capener, C. E., \& Biggin, P. C. (2002). Potassium channels: Structures, models, simulations. Biochimica et Biophysica Acta-Biomembranes, $1565(2)$, 294-307. https://doi.org/10.1016/S0005-2736(02)00576-X

Sarkar, P., Reichman, C., Saleh, T., Birge, R. B., \& Kalodimos, C. G. (2007). Proline cis-trans Isomerization Controls Autoinhibition of a Signaling Protein. Molecular Cell, 25(3), 413-426. https://doi.org/10.1016/j.molcel.2007.01.004

Schroeder, I. (2015). How to resolve microsecond current fluctuations in single ion channels: The power of beta distributions. Channels, 9(5), 262-280. https://doi.org/10.1080/19336950.2015.1083660

Schroeder, I., \& Hansen, U.-P. (2007). Saturation and microsecond gating of current indicate depletioninduced instability of the Maxik selectivity filter. The Journal of General Physiology, 130(1), 83-97. https://doi.org/10.1085/jgp.200709802

Schroeder, I., \& Hansen, U.-P. (2006). Strengths and limits of beta distributions as a means of reconstructing the true single-channel current in patch clamp time series with fast gating. Journal of Membrane Biology, $210(3), 199-212$. https://doi.org/10.1007/s00232-006-0858-8

Schuster, B., Sleytr, U. B., Diederich, A., Bähr, G., \& Winterhalter, M. (1999). Probing the stability of S-layersupported planar lipid membranes. European Biophysics Journal, 28(7), 583-590. https://doi.org/10.1007/s002490050240

Seddon, A. M., Curnow, P., \& Booth, P. J. (2004). Membrane proteins, lipids and detergents: Not just a soap opera. Biochimica et Biophysica Acta - Biomembranes, 1666(1-2), $105-117$. https://doi.org/10.1016/j.bbamem.2004.04.011

Shatzman, A. R. (1990). Gene expression using Gram-negative bacteria. Current Opinion in Biotechnology, 1 (1), 5-1 1. https://doi.org/10.1016/0958-1669(90)90004-5

Shim, J. W., Yang, M., \& Gu, L. Q. (2007). In vitro synthesis, tetramerization and single channel characterization of virus-encoded potassium channel KcV. FEBS Letters, 581(5), 1027-1034. https://doi.org/10.1016/j.febslet.2007.02.005

Shimizu, K., Cao, W., Saad, G., Shoji, M., \& Terada, T. (2018). Comparative analysis of membrane protein structure databases. Biochimica et Biophysica Acta - Biomembranes, 1860(5), 1077-1091. https://doi.org/10.1016/j.bbamem.2018.01.005

Sievers, F., Wilm, A., Dineen, D., Gibson, T. J., Karplus, K., Li, W., Lopez, R., McWilliam, H., Remmert, M., Söding, J., Thompson, J. D., \& Higgins, D. G. (2011). Fast, scalable generation of high-quality protein multiple sequence alignments using Clustal Omega. Molecular Systems Biology, 7(539). https://doi.org/10.1038/msb.201 1.75 
Singer, S. J., \& Nicolson, G. L. (1972). The Fluid Mosaic Model of the Structure of Cell Membranes. Science, $175(4023), 720-731$.

Siotto, F. (2017). Mining and analysis of new viral potassium channel proteins. PhD Thesis, Technische Universität Darmstadt.

Sobhanifar, S., Reckel, S., Junge, F., Schwarz, D., Kai, L., Karbyshev, M., Löhr, F., Bernhard, F., \& Dötsch, V. (2010). Cell-free expression and stable isotope labelling strategies for membrane proteins. Journal of Biomolecular NMR, 46 (1), 33-43. https://doi.org/10.1007/s 10858-009-9364-5

Spector, A. A., \& Yorek, M. A. (1985). Membrane lipid composition and cellular function. Journal of Lipid Research, 26(9), 1015-1035.

Splitt, H., Meuser, D., Borovok, I., Betzler, M., \& Schrempf, H. (2000). Pore mutations affecting tetrameric assembly and functioning of the potassium channel KCSA from Streptomyces lividans. FEBS Letters, 472 (1), 83-87. https://doi.org/10.1016/S0014-5793(00)01429-0

Starkus, J. G., Varga, Z., Schönherr, R., \& Heinemann, S. H. (2003). Mechanisms of the inhibition of Shaker potassium channels by protons. Pflugers Archiv European Journal of Physiology, 447(1), 44-54. https://doi.org/10.1007/s00424-003-1121-0

Steinberg, I. Z., Harrington, W. F., Berger, A., Sela, M., \& Katchalski, E. (1960). The Configurational Changes of Poly-L-proline in Solution. Journal of the American Chemical Society, 82(20), 5263-5279. https://doi.org/10.1021/ja01505a001

Stillwell, W. (2016). An Introduction to Biological Membranes (2nd Editio).

Takahashi, T., Nakamura, H., \& Wada, A. (1992). Electrostatic forces in two lysozymes: Calculations and measurements of histidine pKa values. Biopolymers, 32(8), 897-909. https://doi.org/10.1002/bip.360320802

Tapper, A. R., \& George, A. L. (2003). Heterologous expression of ion channels. Methods in Molecular Biology (Clifton, N.J.), 217, 285-294. https://doi.org/10.1385/1-59259-330-5:285

Tayefeh, S., Kloss, T., Kreim, M., Gebhardt, M., Baumeister, D., Hertel, B., Richter, C., Schwalbe, H., Moroni, A., Thiel, G., \& Kast, S. M. (2009). Model development for the viral Kcv potassium channel. Biophysical Journal, 96 (2), 485-498. https://doi.org/10.1016/j.bpj.2008.09.050

Tayefeh, S., Kloss, T., Thiel, G., Hertel, B., Moroni, A., \& Kast, S. M. (2007). Molecular dynamics simulation of the cytosolic mouth in Kcv-type potassium channels. Biochemistry, 46(16), 4826-4839. https://doi.org/10.1021/bi602468r

Tempel, B. L., Papazian, D. M., Schwarz, T. L., Jan, Y. N., \& Jan, L. Y. (1987). Sequence of a probable potassium channel component encoded at Shaker locus of Drosophila. Science, 237(4816), 770775. https://doi.org/10.1126/science.2441471

Thiel, G., Baumeister, D., Schroeder, I., Kast, S. M., Van Etten, J. L., \& Moroni, A. (201 1). Minimal art: Or why small viral $\mathrm{K}+$ channels are good tools for understanding basic structure and function relations. Biochimica et Biophysica Acta - Biomembranes, 1808(2), 580-588. https://doi.org/10.1016/j.bbamem.2010.04.008

Thiel, G., Moroni, A., Blanc, G., \& Van Etten, J. L. (2013). Potassium Ion Channels: Could They Have Evolved from Viruses? Plant Physiology, 162 (3), 1215-1224. https://doi.org/10.1 104/pp.113.219360

Thompson, A. N., Posson, D. J., Parsa, P. V., \& Nimigean, C. M. (2008). Molecular mechanism of pH sensing in KcsA potassium channels. Proceedings of the National Academy of Sciences of the United States of America, 105(19), 6900-6905. https://doi.org/10.1073/pnas.0800873105

Tien, H. T., Salamon, Z., \& Ottova, A. (1991). Lipid bilayer-based sensors and biomolecular electronics. Critical Reviews in Biomedical Engineering, 18(5), 323-340. http://europepmc.org/abstract/MED/2036800

Tolokh, I. S., Tolokh, I. I., Cho, H. C., D'Avanzo, N., Backx, P. H., Goldman, S., \& Gray, C. G. (2005). NonMichaelis-Menten kinetics model for conductance of low-conductance potassium ion channels. Physical Review E - Statistical, Nonlinear, and Soft Matter Physics, 71(2), 1-9. 
https://doi.org/10.1 103/PhysRevE.71.021912

Valiyaveetil, F. I., Zhou, Y., \& MacKinnon, R. (2002). Lipids in the structure, folding, and function of the KcsA K+ channel. Biochemistry, 41 (35), 10771-10777. https://doi.org/10.1021/bi026215y

Van Etten, J. L. (2003). Unusual life style of giant chlorella viruses. Annual Review of Genetics, 37, $153-195$. https://doi.org/10.1146/annurev.genet.37.110801.143915

Wallin, E., \& Heijne, G. Von. (1998). Genome-wide analysis of integral membrane proteins from eubacterial, archaean, and eukaryotic organisms. Protein Science, 7(4), 1029-1038. https://doi.org/10.1002/pro.5560070420

Wang, C., Takeuchi, K., Pinto, L. H., \& Lamb, R. A. (1993). Ion channel activity of influenza A virus M2 protein: characterization of the amantadine block. Journal of Virology, 67(9), 5585-5594. https://doi.org/10.1128/jvi.67.9.5585-5594.1993

Wedemeyer, W. J., Welker, E., \& Scheraga, H. A. (2002). Proline cis-trans isomerization and protein folding. Biochemistry, 41 (50), 14637-14644. https://doi.org/10.1021/bi020574b

Whited, A. M., \& Johs, A. (2015). The interactions of peripheral membrane proteins with biological membranes. Chemistry and Physics of Lipids, 192, 51-59. https://doi.org/10.1016/j.chemphyslip.2015.07.015

Williams, A. J. (1994). An introduction to the methods available for ion channel reconstitution. Microelectrode Techniques, 79-99. https://doi.org/10.1021/la3041506

Winterstein, L.-M. (2019). Einflüsse verschiedener Membranen auf die viralen Kaliumkanäle Kcv NTS , KcV S und Kmpv 12T. PhD Thesis, Technische Universität Darmstadt.

Winterstein, L.-M., Kukovetz, K., Rauh, O., Turman, D. L., Braun, C., Moroni, A., Schroeder, I., \& Thiel, G. (2018). Reconstitution and functional characterization of ion channels from nanodiscs in lipid bilayers. Journal of General Physiology, 150(4), 637-646. https://doi.org/10.1085/jgp.201711904

Winterstein, T. (2019). Quaternary ammonium cations are a useful tool to investigate viral potassium channels. PhD Thesis, Technische Universität Darmstadt.

Zakharian, E. (2013). Recording of ion channel activity in planar lipid bilayer experiments. In Methods in Molecular Biology (Vol. 998). Humana Press, Totowa, NJ. https://doi.org/10.1007/978-1-62703-351-0_8

Zhou, M., \& MacKinnon, R. (2004). A mutant KcsA K+ channel with altered conduction properties and selectivity filter ion distribution. Journal of Molecular Biology, 338(4), 839-846. https://doi.org/10.1016/j.jmb.2004.03.020

Zhou, M., Morais-Cabral, J. H., Mann, S., \& MacKinnon, R. (2001). Potassium chanel receptor site for the inactivation gate and quaternary amine inhibitors. Nature, 411, 657-661.

Zhou, Y., Morais-Cabral, J. H., Kaufman, A., \& MacKinnon, R. (2001). Chemistry of ion coordination and hydration revealed by a K+ channel-Fab complex at 2.0 A resolution. Nature, 414 (6859), 43-48. https://doi.org/10.1038/35102009

Zhu, L., Kemple, M. D., Yuan, P., \& Prendergast, F. G. (1995). N-Terminus and Lysine Side Chain pKa Values of Melittin in Aqueous Solutions and Micellar Dispersions Measured by 15N NMR. Biochemistry, 34 (40), 13196-13202. https://doi.org/10.1021/bi00040a035

Zimmerman, S. S., \& Scheraga, H. A. (1976). Stability of Cis, Trans, and Nonplanar Peptide Groups. Macromolecules, 9(3), 408-416. https://doi.org/10.1021/ma60051a005 


\section{Appendix}

\section{$5.1 \quad$ Figures}

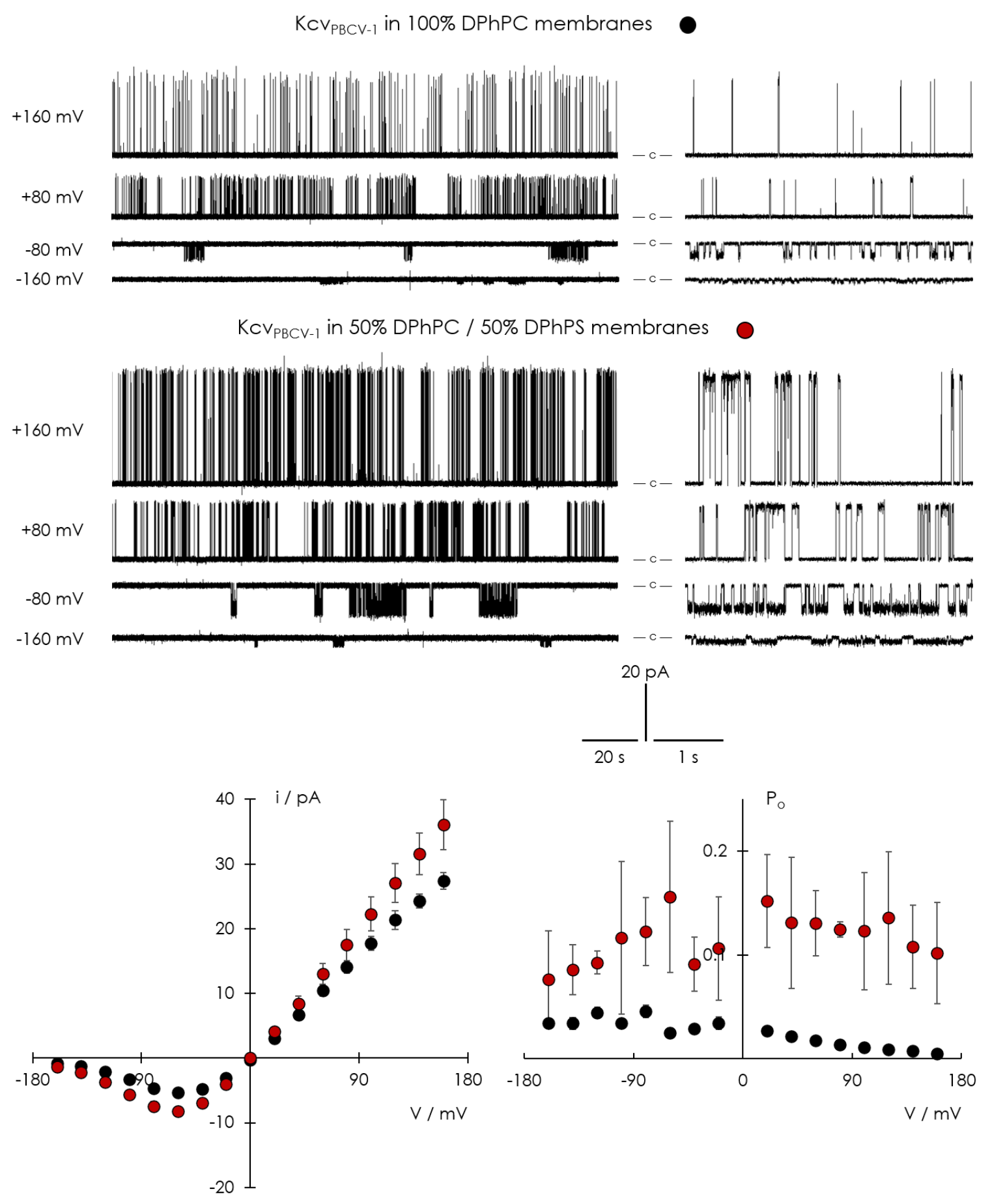


Figure 44 - KCVPBCV-1 in different bilayers. (A) Representative single channel traces (left) of KCVPBCV-1 in pure DPhPC membranes from $+160 \mathrm{mV}$ to $-160 \mathrm{mV}$ in $80 \mathrm{mV}$ steps. Exemplary extracts with a higher temporal resolution of the same measurement are shown on the right side, for better visualization of individual gating events. c marks the closed state. For better visualization, data was subsequently filtered with $500 \mathrm{~Hz}$. (B) Representative single channel traces (left) of KCVPBCV-1 in membranes consisting of $50 \%$ DPhPC and $50 \%$ DPhPS from $+160 \mathrm{mV}$ to $-160 \mathrm{mV}$ in $80 \mathrm{mV}$ steps. Exemplary extracts with a higher temporal resolution of the same measurement are shown on the right side, for better visualization of individual gating events. $c$ marks the closed state. Corresponding (C) i/V curve and (D) open probability curve of KCVPBCV-1 in different membranes. Colors correspond to those from (A) and (B). Data are mean values \pm standard deviation for $\mathrm{n}$ independent measurements (KCVPBCV-1 in DPhPC: $n=8 ; \mathrm{KCVPBCV}_{-1}$ in DPhPC/DPhPS: $n=6$ ). Measurements were performed under symmetrical conditions with $100 \mathrm{mM} \mathrm{KCl}$ and $10 \mathrm{mM} \mathrm{HEPES}$ at $\mathrm{pH} 7$.

\subsection{Tables}

Table 2 - Values for the parameters of equation (10) that result in the best fit

\begin{tabular}{c|c} 
Parameter & Best fit \\
\hline $\mathrm{k}^{*}\left[\mathrm{~s}^{-1} \mathrm{M}^{-\mathrm{h}}\right]$ & 3.744 \\
\hline $\mathrm{k}-1\left[\mathrm{~s}^{-1}\right]$ & 0.025 \\
\hline $\mathrm{k}^{*} 2\left[\mathrm{~s}^{-1} \mathrm{M}^{-\mathrm{h}}\right]$ & 20701.558 \\
\hline $\mathrm{k}_{-2}\left[\mathrm{~s}^{-1}\right]$ & 2.698 \\
\hline $\mathrm{G}_{\max }[\mathrm{pS}]$ & 202.876 \\
\hline $\mathrm{G}_{\min }[\mathrm{pS}]$ & 25.461 \\
\hline $\mathrm{h}_{1}$ & 0.418 \\
\hline $\mathrm{h}_{2}$ & 0.549 \\
\hline$\chi^{2}$ & 15.954
\end{tabular}

\subsection{List of abbreviations}

\section{Amino acids}

$\begin{array}{lll}\text { A } & \text { Ala } & \text { Alanine } \\ \text { C } & \text { Cys } & \text { Cysteine } \\ \text { D } & \text { Asp } & \text { Aspartic acid (aspartate) } \\ \text { E } & \text { Glu } & \text { Glutamic acid (glutamate) } \\ \text { F } & \text { Phe } & \text { Phenylalanine } \\ \text { G } & \text { Gly } & \text { Glycine } \\ \text { H } & \text { His } & \text { Histidine } \\ \text { I } & \text { He } & \text { Isoleucine } \\ \text { K } & \text { Lys } & \text { Lysine }\end{array}$




$\begin{array}{lll}\text { L } & \text { Leu } & \text { Leucine } \\ \text { M } & \text { Met } & \text { Methionine } \\ \text { N } & \text { Asn } & \text { Asparagine } \\ \text { P } & \text { Pro } & \text { Proline } \\ \text { Q } & \text { Gln } & \text { Glutamine } \\ \text { R } & \text { Arg } & \text { Arginine } \\ \text { S } & \text { Ser } & \text { Serine } \\ \text { T } & \text { Thr } & \text { Threonine } \\ \text { V } & \text { Val } & \text { Valine } \\ \text { W } & \text { Trp } & \text { Tryptophan } \\ \text { Y } & \text { Tyr } & \text { Tyrosine }\end{array}$

\subsection{Primer sequences}

\begin{tabular}{|c|c|c|}
\hline KcV L94A & $\begin{array}{l}\text { fW } \\
\text { rev }\end{array}$ & $\begin{array}{l}\text { GTTCTTCATCGTTCTAACTGCATAGAAGGGTC } \\
\text { GGTGATGATGACCCTTCTATGCAGTTAGAACG }\end{array}$ \\
\hline Kcv L94C & $\begin{array}{l}\text { fw } \\
\text { rev }\end{array}$ & $\begin{array}{l}\text { GTTCTTCATCGTTCTAACTTGCTAGAAGGG } \\
\text { GGTGATGATGACCCTTCTAGCAAGTTAGAACG }\end{array}$ \\
\hline KcVL94D & $\begin{array}{l}\text { fW } \\
\text { rev }\end{array}$ & $\begin{array}{l}\text { GTTCTTCATCGTTCTAACTGACTAGAAGGGTC } \\
\text { GGTGATGATGACCCTTCTAGTCAGTTAGAACG }\end{array}$ \\
\hline KcvL94E & $\begin{array}{l}\text { fw } \\
\text { rev }\end{array}$ & $\begin{array}{l}\text { CTTCATCGTTCTAACTGAATAGAAGGGTC } \\
\text { GATGATGACCCTTCTATTCAGTTAGAACG }\end{array}$ \\
\hline KcvL94F & $\begin{array}{l}\text { fw } \\
\text { rev }\end{array}$ & $\begin{array}{l}\text { GTTCTTCATCGTTCTAACTTTTTAGAAGGGTC } \\
\text { GGTGATGATGACCCTTCTAAAAAGTTAGAACG }\end{array}$ \\
\hline KcvL94G & $\begin{array}{l}\text { fw } \\
\text { rev }\end{array}$ & $\begin{array}{l}\text { GTTCTTCATCGTTCTAACTGGATAGAAGGGTC } \\
\text { GGTGATGATGACCCTTCTATCCAGTTAGAACG }\end{array}$ \\
\hline KcV L94H & $\begin{array}{l}\text { fw } \\
\text { rev }\end{array}$ & $\begin{array}{l}\text { GTTCTTCATCGTTCTAACTCACTAGAAGGGTC } \\
\text { GGTGATGATGACCCTTCTAGTGAGTTAGAACG }\end{array}$ \\
\hline Kcv L94I & $\begin{array}{l}\text { fw } \\
\text { rev }\end{array}$ & $\begin{array}{l}\text { GTTCTTCATCGTTCTAACTATATAGAAGGGTC } \\
\text { GGTGATGATGACCCTTCTATATAGTTAGAACG }\end{array}$ \\
\hline KcVL94K & $\begin{array}{l}\text { fw } \\
\text { rev }\end{array}$ & $\begin{array}{l}\text { CTTCATCGTTCTAACTAAATAGAAGGGTC } \\
\text { GATGATGACCCTTCTATTTAGTTAGAACG }\end{array}$ \\
\hline KCV L94M & $\begin{array}{l}\text { fW } \\
\text { rev }\end{array}$ & $\begin{array}{l}\text { GTTCTTCATCGTTCTAACTATGTAGAAGGGTC } \\
\text { GGTGATGATGACCCTTCTACATAGTTAGAACG }\end{array}$ \\
\hline KcvL94N & $\begin{array}{l}\text { fw } \\
\text { rev }\end{array}$ & $\begin{array}{l}\text { GTTCTTCATCGTTCTAACTAACTAGAAGGGTC } \\
\text { GGTGATGATGACCCTTCTAGTTAGTTAGAACG }\end{array}$ \\
\hline KCV L94P & $\begin{array}{l}\text { fw } \\
\text { rev }\end{array}$ & $\begin{array}{l}\text { GTTCTTCATCGTTCTAACTCCATAGAAGGGTC } \\
\text { GGTGATGATGACCCTTCTATGGAGTTAGAACG }\end{array}$ \\
\hline KcvL94Q & $\begin{array}{l}\text { fw } \\
\text { rev }\end{array}$ & $\begin{array}{l}\text { GTGTTCTTCATCGTTCTAACTCAATAGAAGGG } \\
\text { GGTGATGATGACCCTTCTATTGAGTTAGAACGATG }\end{array}$ \\
\hline
\end{tabular}




\begin{tabular}{|c|c|c|}
\hline KcVL94R & $\begin{array}{l}\text { fw } \\
\text { rev }\end{array}$ & $\begin{array}{l}\text { GTTCTTCATCGTTCTAACTAGATAGAAGGGTC } \\
\text { GGTGATGATGACCCTTCTATCTAGTTAGAACG }\end{array}$ \\
\hline KcVL94S & $\begin{array}{l}\text { fw } \\
\text { rev }\end{array}$ & $\begin{array}{l}\text { GTTCTTCATCGTTCTAACTTCATAGAAGGGTC } \\
\text { GGTGATGATGACCCTTCTATGAAGTTAGAACG }\end{array}$ \\
\hline KcV L94T & $\begin{array}{l}\text { fW } \\
\text { rev }\end{array}$ & $\begin{array}{l}\text { GTTCTTCATCGTTCTAACTACATAGAAGGGTC } \\
\text { GGTGATGATGACCCTTCTATGTAGTTAGAACG }\end{array}$ \\
\hline KcV L94V & $\begin{array}{l}\text { fw } \\
\text { rev }\end{array}$ & $\begin{array}{l}\text { GTTCTTCATCGTTCTAACTGTATAGAAGGGTC } \\
\text { GGTGATGATGACCCTTCTATACAGTTAGAACG }\end{array}$ \\
\hline KcVL94W & $\begin{array}{l}\text { fw } \\
\text { rev }\end{array}$ & $\begin{array}{l}\text { GTTCTTCATCGTTCTAACTTGGTAGAAGGGTC } \\
\text { GGTGATGATGACCCTTCTACCAAGTTAGAACG }\end{array}$ \\
\hline KCVL94Y & $\begin{array}{l}\text { fw } \\
\text { rev }\end{array}$ & $\begin{array}{l}\text { GTTCTTCATCGTTCTAACTTACTAGAAGGGTC } \\
\text { GGTGATGATGACCCTTCTAGTAAGTTAGAACG }\end{array}$ \\
\hline KcVV64T & $\begin{array}{l}\text { fw } \\
\text { rev }\end{array}$ & $\begin{array}{l}\text { GTAACGACACACTCTACTACCGGATTCGG } \\
\text { GGCAGTATATCTCCGAATCCGGTAGTAGAGTG }\end{array}$ \\
\hline $\mathrm{KCV}_{\text {MT325 }} \mathrm{T55 \textrm {V }}$ & $\begin{array}{l}\text { fw } \\
\text { rev }\end{array}$ & $\begin{array}{l}\text { GGCCGCCACACACACAACAGTAGGTTTTGG } \\
\text { CCACAATATCTCCAAAACCTACTGTTGTGTGTG }\end{array}$ \\
\hline KCVGNLDT56V & $\begin{array}{l}\text { fW } \\
\text { rev }\end{array}$ & $\begin{array}{l}\text { CGGCAACGCACACCACCGTCGGGTTTG } \\
\text { GGGACTACATCACCAAACCCGACGGTGGTG }\end{array}$ \\
\hline
\end{tabular}




\section{List of figures}

Figure 1-Schematic illustration of the amount of TMDs in different subunits of $\mathrm{K}^{+}$channels........................

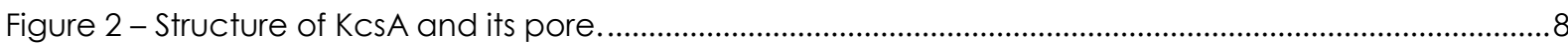

Figure 3 - Different views on the structure of the bacterial channel KcsA............................................... 10

Figure 4 - Schematic illustration of the different types of inactivation........................................................ 12

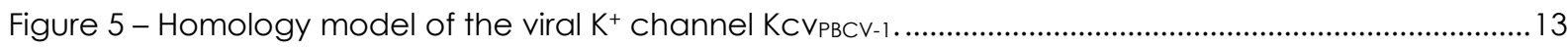

Figure 6 - Sequence alignment of a selection of KCV channels. .............................................................15

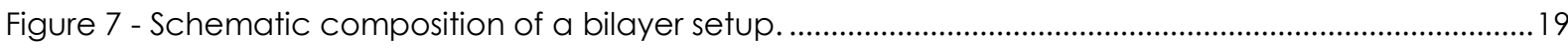

Figure 8 - Reconstitution of the viral $\mathrm{K}^{+}$channel $\mathrm{KCV}_{\mathrm{NTS}}$ from different NDs in pure DPhPC membranes....23

Figure 9 - Reconstitution of the bacterial $\mathrm{K}^{+}$channel KcSA E7 1 A in PLBs with different membranes.........25

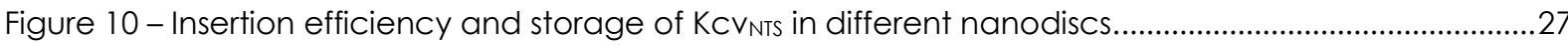

Figure 11 - Single channel properties of the blue-light-induced $\mathrm{K}^{+}$channel 1 (BLINK1) compared to KCVPBCV1

Figure 12 - Single channel properties of BLINK1 after blue light exposure.................................................30

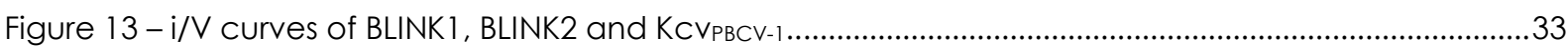

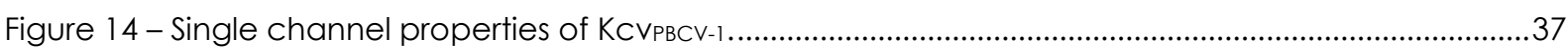

Figure 15 - Open probability of KCVPBCV-1 and KCVPBCV-1 L94X as a function of physicochemical properties

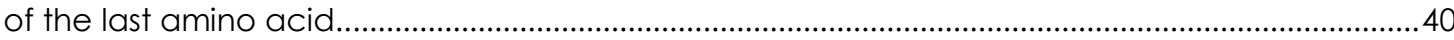

Figure 16 - Unitary single channel conductance of KCVPBCV-1 and KCVPBCV-1 L94X...................................41

Figure 17 - Unitary single channel conductance of KCVPBCV-1 and KCVPBCV-1 L94X as a function of

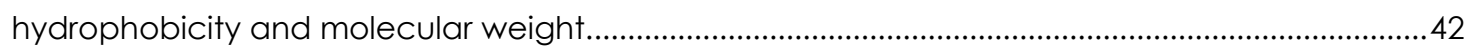

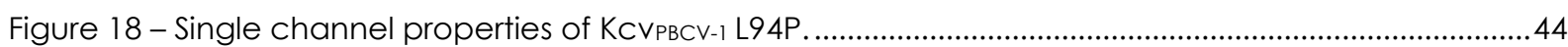

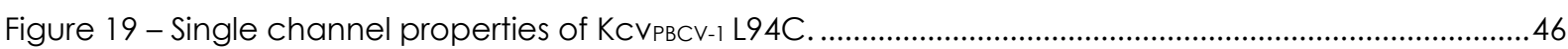

Figure 20 - Difference between the Po value at -160 mV and +160 mV for KCVPBCV-1 (Wt) and KCVPBCV-1 L94C.

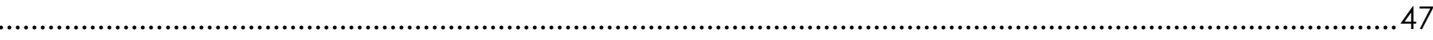

Figure 21 - Comparison of KCVPBCV-1 and mutants L94H, L94K and L94R...............................................49

Figure 22 - Comparison of KCVPBCV-1 L94H at different pH values............................................................52

Figure 23 - Unitary single channel conductance of KCVPBCV-1 L94H as a function of $\mathrm{H}^{+}$concentration......53

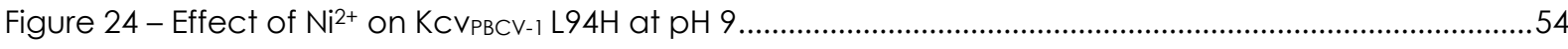

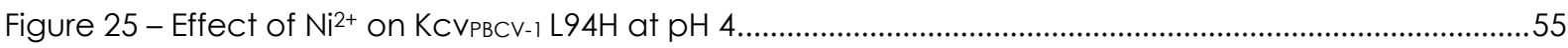

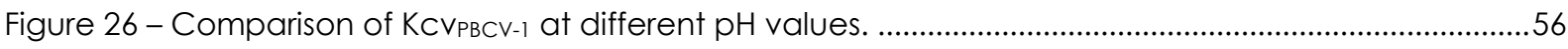

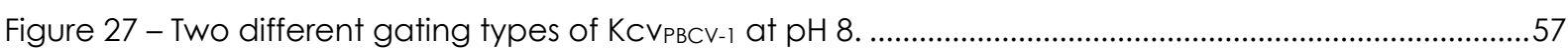

Figure 28 - Unitary single channel conductance of KCVPBCV-1 and KCVPBCV-1 $\mathrm{K} 94 \mathrm{H}$ as a function of $\mathrm{H}^{+}$ concentration. 
Figure 29 - Yeast complementation assays of the strain SGY1528 transformed with pYES2, KCVPBCV-1 and KCVPBCV-1 L94X.

Figure 30 - Simple two-state model describes the effect of $\mathrm{pH}$ on KCVPBCV-1 and KCVPBCV-1 L94H. .65

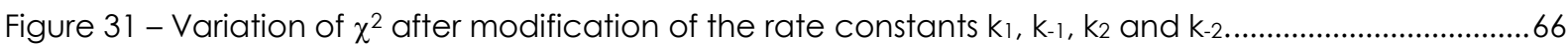

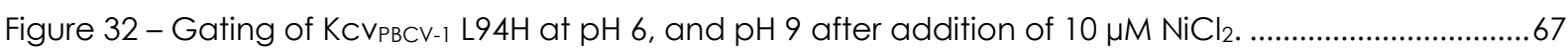

Figure 33 - Comparison of the two different gating types of BLINK 1 and KCVPBCV-1 at $\mathrm{pH} 8$......................69

Figure 34 - Alignment of the viral K+ channels KCVPBCV-1, KCVGNLD and KCVMT325 .......................................71

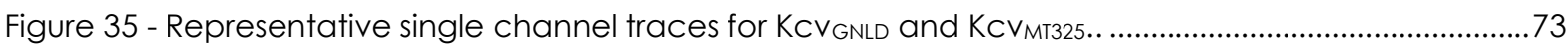

Figure 36 - i/ $\mathrm{V}$ curves and single channel conductance of KCVGNLD and KCVMT325 for different $\mathrm{K}^{+}$

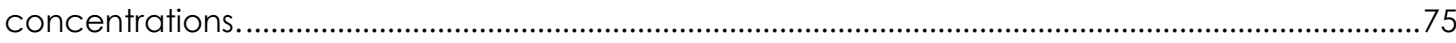

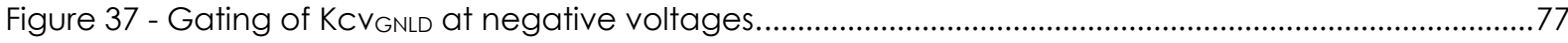

Figure 38 - Voltage-dependent open probability of KCVGNLD and KCVMT325 as a function of $\mathrm{K}^{+}$ concentrations.....

Figure 39 - Alignment of the signature sequences of several viral $\mathrm{K}^{+}$channels and the different amino acids at position 5. 79

Figure 40 - Representative single channel traces for KCVGNLD T56V and KCVMT325 T55V. . .81

Figure 41 - i/V curves, single channel conductance and open probability of KCVGNLD T56V and KCVMT325 T55V compared to the wild type channels. . .83

Figure 42 - Representative single channel traces for KCVPBCV-1 and KCVPBCV-1 V64T. . .85

Figure 43 - i/V and open probability curves of KCVPBCV-1 and KCVPBCV-1 V64T. . .86

Figure 44 - KCVPBCV-1 in different bilayers. . .99 


\section{Own work}

All experiments, data analyses and writing of the present thesis were performed by myself, with the following exceptions:

- Chapter 3.1.1 "Target membrane properties determine channel function" was already published in a similar form in Winterstein et al., 2018

- In chapter 3.2.2 The generation, measurements and data analyses of KCVPBCV-1 L94X mutants was done together with Matea Cartolano (Master student) under my supervision.

- In chapter 3.2.4 measurements of KCVPBCV-1 L94C with DTT were performed by Matea Cartolano (Master student) under my supervision.

- In chapter 3.2.6 some measurements of $\mathrm{KCVPBCV}_{\mathrm{I}}$ at $\mathrm{pH} 4$ and $\mathrm{pH}$ 9, as well as measurements of KCVPBCV-1 $\mathrm{L} 94 \mathrm{H}$ with addition of $\mathrm{NiCl}_{2}$ were performed by Jonas Stein (Bachelor student) under my supervision.

- Yeast complementation assays from fig. 29 in chapter 3.2.7 were performed by Manuela Gebhardt (unpublished data, TU Darmstadt).

- Construction of a two-state model and the underlying mathematical framework in chapter 3.2.7 was kindly constructed by Dr. Oliver Rauh.

- In chapter 3.3 some measurements of KCVGNLD, KCVMT325, KCVGNLD T56V and KCVMT325 T55V were performed within the master course during by the students Ute Korn and Lukas Neuenfeld, under my close supervision. 


\section{Declaration / Ehrenwörtliche Erklärung}

Ich erkläre hiermit ehrenwörtlich, dass ich die vorliegende Arbeit entsprechend den Regeln guter wissenschaftlicher Praxis selbstständig und ohne unzulässige Hilfe Dritter angefertigt habe.

Sämtliche aus fremden Quellen direkt oder indirekt übernommenen Gedanken sowie sämtliche von Anderen direkt oder indirekt übernommenen Daten, Techniken und Materialien sind als solche kenntlich gemacht. Die Arbeit wurde bisher bei keiner anderen Hochschule zu Prüfungszwecken eingereicht.

Darmstadt, den 10.06.2020

Kerri Kukovetz 


\section{Curriculum vitae and publications}

Personal Information

$\begin{array}{ll}\text { First name } & \text { Kerri } \\ \text { Last name } & \text { Kukovetz } \\ \text { Date of birth } & 18.03 .1988 \\ \text { Place of birth } & \text { Aschaffenburg } \\ \text { Nationality } & \text { irish / austrian }\end{array}$

Languages

German (native)

English (native)

Education

Sep 2016 - May 2020

Doctoral degree candidate at the Technical University of Darmstadt in the working group of Prof. Dr. Gerhard Thiel (Membrane Biophysics)

Doctoral advisor: Prof. Dr. Gerhard Thiel

$2014-\operatorname{Sep} 2016$

Study of Technical Biology at the Technical University of Darmstadt

Courses: Radiation Biology, Cellular Biophysics, Neurobiology, immunocytochemical and electrophysiological methods for examination of $\mathrm{K}^{+}$ channels in neuronal stem cells (alternative course at the working group of Prof. Dr. Bodo Laube)

Master-Thesis: "pH-dependent gating mechanism of the viral potassium channel KCVNTS" in the working group of Prof. Dr. Gerhard Thiel (Membrane Biophysics)

Degree: Master of Science

$2010-2014$

\section{Study of Biology at the Technical University of Darmstadt}

Advanced courses: Animal Physiology, Molecular Developmental Biology, Radiation Biology, Biophysics of ion transport

Bachelor-Thesis: "pH-dependency of the viral $\mathrm{K}^{+}$channel KcVNTs" in the working group of Prof. Gerhard Thiel (Membrane Biophysics)

Degree: Bachelor of Science 
$2008-2010$
University of Applied Sciences Darmstadt, Study of Electrical Engineering and Information Technology

Major subject: Telecommunication

Franziskanergymnasium Kreuzburg, Großkrotzenburg

Abitur

Work experience

Since Sep 2016

Apr $2014-$ Sep 2016

Sep 2007 - Mär 2014

Apr 2006 - Aug 2007
Minijob as Sales Advisor for the company Hennes \& Mauritz in Darmstadt

Sales Advisor for the company Hennes \& Mauritz in Darmstadt

Sales Advisor for the company Hennes \& Mauritz in Aschaffenburg

Temporary worker for the company "Ecoplan"

Publications

Submitted

Winterstein, L.-M., Kukovetz, K., Hansen, U.-P., Schroeder, I., Van Etten, J., Moroni, A., Thiel, G., Rauh, O. General and protein specific effects of bilayer composition on K+ channel function. J Gen Physiol.

$06 / 2020$

Murry, C., Agarkova, I. V., Ghosh, j. S., Fitzgerald F. C., Carlson R. M., Hertel B., Kukovetz, K., Rauh, Ol., Thiel, G., Van Etten, J. (2020). Genetic Diversity of Potassium Ion Channel Proteins Encoded by Chloroviruses that Infect Chlorella heliozoae. Viruses, 12(6), 678. https://doi.org/10.3390/v12060678

$10 / 2018$

Alberio, L., Locarno, A., Saponaro, A., Romano, E., Bercier, V., Albadri, S., Simeoni, F., Moleri, S., Pelucchi, S., Porro, A., Marcello, E., Barsotti, N., Kukovetz, K. Boender, A. J., Contestabile, A., Luo, S., Moutal, A., Ji, Y., Romani, G., Beltrame, M., Del Bene, F., Di Luca, M., Khanna, R., Colecraft, H. M., Pasqualetti, M., Thiel, G., Tonini R., Moroni, A. (2018). A light-gated potassium channel for sustained neuronal inhibition. Nature Methods, 15, 969-976. https://doi.org/10.1038/s41592-018-01869

Winterstein, L.-M., Kukovetz, K., Rauh, O., Turman, D. L., Braun, C., Moroni, A., Schroeder, I., Thiel, G. (2018). Reconstitution and functional characterization of ion channels from nanodiscs in lipid bilayers. J Gen Physiol, 150(4), 637-646. https://doi.org/10.1085/jgp.201711904

L.-M. Winterstein, K. Kukovetz and O. Rauh contributed equally to this paper. 


\section{Acknowledgements}

An dieser Stelle möchte ich mich bei all den Menschen bedanken, die in den vergangenen Jahren für mich da waren, mich unterstütz† und zum Erfolg dieser Arbeit beigetragen haben.

Mein besonderer Dank geht an:

Prof. Dr. Gerhard Thiel, für die Möglichkeit in Deiner Arbeitsgruppe promovieren zu dürfen, so wie für die Unterstützung, die vielen Ratschläge und das stetige Interesse an meiner Arbeit. Auch für die großartige Atmosphäre innerhalb und außerhalb des Labors, die ein sehr angenehmes und spaßiges Arbeitsumfeld geschaffen hat.

PD Dr. Indra Schröder, für die Übernahme des Koreferats, so wie die Beantwortung sämtlicher Fragen insbesondere was das sich willkürlich verändernde Rauschen am Bilayer oder KielPatch/Patchmaster betraf ;-)

Oliver Rauh, dafür, dass Du so viel deiner ohnehin schon wenigen Zeit investiert hast um mir, vor allem am Ende der Arbeit, zu helfen. Abgesehen von der ganzen Hilfe im Labor, beim Beantworten meiner (dummen) Fragen und bei all meinen mathematischen Problemchen, danke ich dir auch für die inzwischen langjährige Freundschaft und die vielen gemeinsamen Aktivitäten, sei es bei Konzerten, beim Fußball schaven, beim Grillen oder beim Genießen des ein oder anderen Bieres (auch international, sláinte!).

Bastian Roth, dass du dir die Zeit genommen hast meine Arbeit Korrektur zu lesen und Fehler zu finden die ich zum hundertsten Mal überlesen habe, oder meine verschachtelten Sätze zu kürzen. Ich freue mich darauf bald wieder mehr Zeit im Biergarten oder Patricks Garten mit dir/euch zu verbringen!

Tanja Musielak and Barbara Reinhards, dafür, dass Ihr es geschafft habt immer Ordnung in das bürokratische Chaos zu bringen.

Brigitte Hertel, Silvia Haase, Sylvia Lenz, Mirja Manthey and Ulrike Burk, dafür, dass ohne Euch der Laboralltag nicht so unkompliziert und reibungslos ablaufen könnte. Auch danke an Mirja und Brigitte, für den ein oder anderen sportlichen Start in den Tag!

Matea Cartolano, dass du so eine angenehme Masterstudentin warst. Deine eigenständige Arbeit im Labor und deine guten Ergebnisse haben mir im Rahmen meiner Arbeit sehr geholfen.

An die gesamte AG Thiel. Laura Winterstein, geb. Schlee, Tobias Schulze, Dominique Tandl, René Lautenschläger, Tobias Winterstein, Sebastian Fuck für viele spaßige Donnerstage (ab 4), die manchmal weniger spaßigen Frühstücke am nächsten Morgen, so wie die vielen Lauf- \& Bouldersessions und viele weitere gemeinsame Aktivitäten. Sebastian Höler, Anja Engel, Deborah Scheub und Paolo Zuccholini, für eine entspannte und "grüne" Büroatmosphäre (Gärtnercredits an Anja und Basti).

Die alte AG Thiel mit Christian Braun, Patrick Voos, Vera Bandmann und Lucia Carillo, schön, dass mit den Jahren aus Arbeit so eine schöne Freundschaft entstanden ist!

Baschtel, Angi, Johanna, Maria, Flo, Daniel, dafür, dass ich Euch in meinem Leben habe 0

Meine Eltern, Mary \& Karl, dafür, dass Ihr nie aufgehört habt an mich zu glauben und mich all die Jahre so sehr unterstützt habt, auch wenn ich immer zu wenig Zeit hatte euch so zu danken, wie Ihr es verdient habt!

Zu guter letz† noch Nesimi Ercan, die wöchentliche Hoffnung den Eurojackpot zu gewinnen hat mir in der Endphase dieser Arbeit sehr geholfen ;-) 